\title{
Advanced contrasts for vascular MRI
}

Citation for published version (APA):

Wolters, M. (2017). Advanced contrasts for vascular MRI. [Doctoral Thesis, Maastricht University]. Maastricht University. https://doi.org/10.26481/dis.20170927mw

Document status and date:

Published: 01/01/2017

DOI:

10.26481/dis.20170927mw

Document Version:

Publisher's PDF, also known as Version of record

\section{Please check the document version of this publication:}

- A submitted manuscript is the version of the article upon submission and before peer-review. There can be important differences between the submitted version and the official published version of record.

People interested in the research are advised to contact the author for the final version of the publication, or visit the DOI to the publisher's website.

- The final author version and the galley proof are versions of the publication after peer review.

- The final published version features the final layout of the paper including the volume, issue and page numbers.

Link to publication

\footnotetext{
General rights rights.

- You may freely distribute the URL identifying the publication in the public portal. please follow below link for the End User Agreement:

www.umlib.nl/taverne-license

Take down policy

If you believe that this document breaches copyright please contact us at:

repository@maastrichtuniversity.nl

providing details and we will investigate your claim.
}

Copyright and moral rights for the publications made accessible in the public portal are retained by the authors and/or other copyright owners and it is a condition of accessing publications that users recognise and abide by the legal requirements associated with these

- Users may download and print one copy of any publication from the public portal for the purpose of private study or research.

- You may not further distribute the material or use it for any profit-making activity or commercial gain

If the publication is distributed under the terms of Article $25 \mathrm{fa}$ of the Dutch Copyright Act, indicated by the "Taverne" license above, 


\section{Advanced contrasts}

for vascular MRI

Martijn Wolters 
Copyright ( 2017 by M. Wolters. All rights reserved. No part of this publication may be reproduced, stored in a retrieval system, or transmitted in any form, or by any means without the prior written permission by the author, or when appropriate, by the publisher of the publications.

Cover design and layout by Martijn Wolters

Printed by ProefschriftMaken 


\section{Advanced contrasts}

\section{for vascular MRI}

\section{PROEFSCHRIFT}

ter verkrijging van de graad van doctor aan de Universiteit Maastricht, op gezag van de Rector Magnificus, Prof.dr. Rianne M. Letschert volgens het besluit van het College van Decanen,

in het openbaar te verdedigen

op woensdag 27 september om 12:00 uur

door

\section{Martijn Wolters}

geboren op 4 april 1981 te Emmen, Nederland 


\section{Promotores}

Prof. dr. M.J. Post

Prof. dr. ir. W.H. Backes

Prof. dr. M.E. Kooi

\section{Beoordelingscommissie}

Prof. dr. F.M. Mottaghy (voorzitter)

Dr. J.M.E.M. Cosemans

Prof. dr. B. Gallez (Université catholique de Louvain)

Prof. dr. A. Heerschap (Radboud Universiteit Nijmegen)

Prof. dr. ir. F. Verhaegen

The studies presented in this thesis were performed within the CARIM School for Cardiovascular Diseases.

Financial support by the Dutch Heart Foundation for the publication of this thesis is gratefully acknowledged.

In addition, finacial support by Maastricht University, Stichting Hartsvrienden RESCAR, and Yacht for the publication of this thesis is gratefully acknowledged. 


\section{Contents}

$\begin{array}{ll}\text { 1. Introduction } & 7\end{array}$

2. Efficacy of positive contrast imaging techniques for molecular MRI of tumor angiogenesis

3. MRI artifacts in the ferric chloride thrombus animal model: an alternative solution

4. Clinical perspectives of hybrid proton-fluorine magnetic resonance imaging and spectroscopy

5. Molecular MRI with a fibrin specific fluorine-loaded polymer nanoparticle

6. Super-resolution reconstruction techniques for ${ }^{19}$ Fluorine MR angiography 101

7. General discussion

Summary

Nederlandstalige samenvatting

Valorisation

Dankwoord

Curriculum Vitea

List of publications 



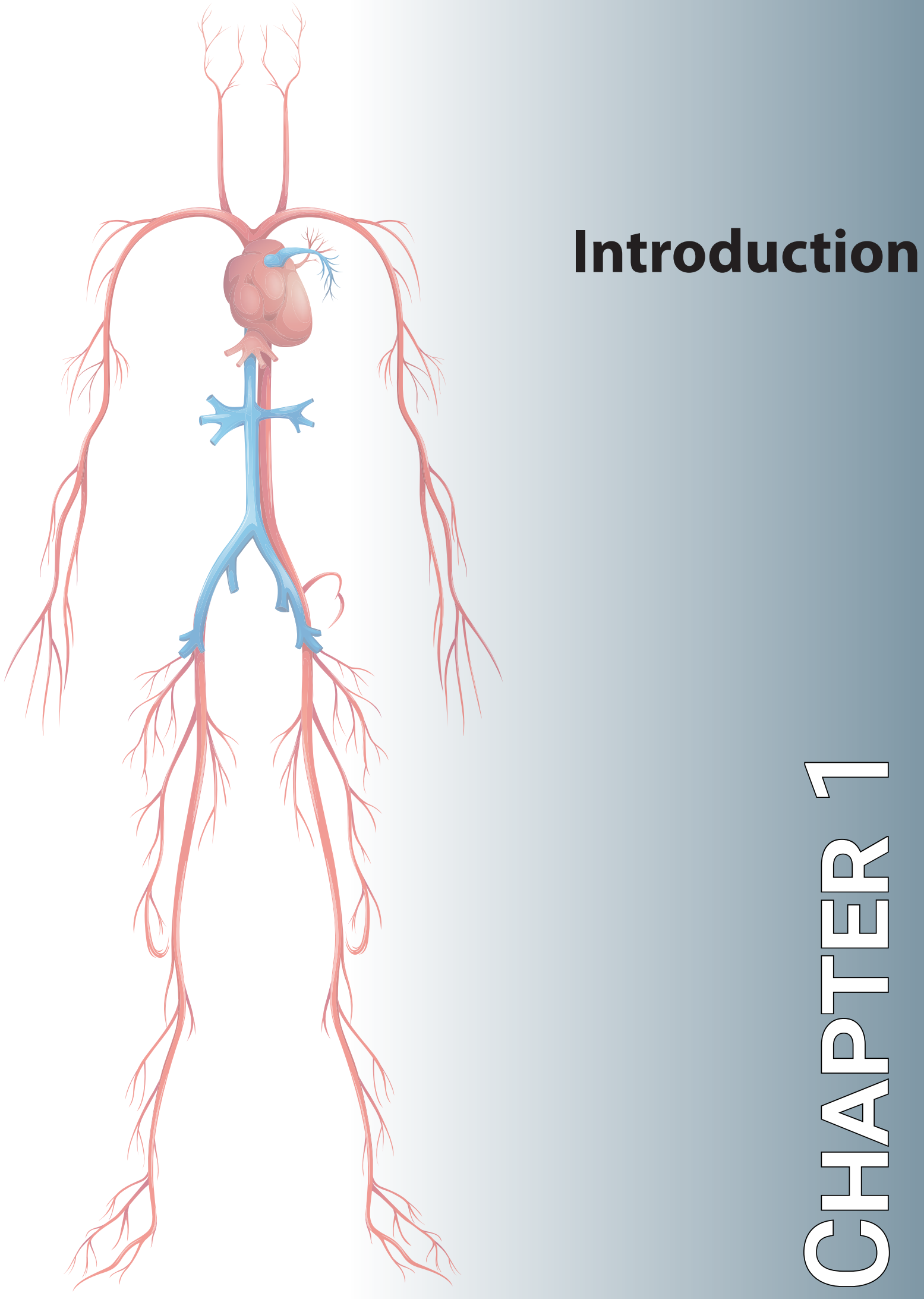


Chapter 1 


\subsection{Introduction}

Magnetic resonance imaging (MRI) is one of the most versatile non-invasive medical imaging modalities available today. Obvious clinical advantages are the high spatial resolution, superior soft tissue contrast, the absence of ionizing radiation and the possibility to perform quantitative measurements, for instance, to determine tissue fat concentration, metabolic composition, (blood and cerebrospinal) fluid flow and water diffusion ${ }^{[1-5]}$. The versatility of MRI can be further extended with the use of contrast agents to enhance contrast between normal and diseased tissue, and to improve visualization of the blood vessels (i.e. angiography).

Gadolinium-based contrast agents are by far the most clinically used MRI contrast agents. They are administered intravenously and therefore enhance contrast in vessels and adjacent tissue. It is therefore extensively applied for cardiovascular imaging and to visualize abnormalities in microvasculature of tumors. In a Gadolinium-based contrast agent (GBCA), gadolinium is incorporated in a chelate which prevents the release of toxic gadolinium ions ${ }^{[6]}$. Various contrast agents and chelates are currently on the market for clinical as well as experimental applications. The molecular composition, structure and size of the contrast agent determine its pharmacokinetic behavior and contrast effect. For blood vessels and the heart, GBCAs are used for the visualization of the vessel lumen (contrast-enhanced MR angiography, CE-MRA) and viability of the myocardium (late-enhancement imaging), respectively ${ }^{[7]}$. CE-MRA can solve imaging problems in non-contrast enhanced MR angiography protocols (e.g. time-of-flight, TOF), such as disturbed inflow of unsaturated blood. In fact, MR angiography without contrast agents can be unreliable in patients with vascular diseases, where the blood flow is often low and non-laminar near the vascular lesion. GBCAs can also reveal physiologic conditions of organs or tissue, such as (micro)vascular perfusion and permeability. The permeability or leakiness of microvessels in tumors is related to tumor malignancy ${ }^{[8]}$. Leakiness is also used in neuroimaging to assess the integrity of the blood-brain barrier, where contrast enhancement indicates increased vascular permeability in, for instance, brain infarctions and (high-grade) tumors. Perfusion MRI provides physiological information about blood volume, distribution and flow. The first pass of a contrast agent after injection is imaged with a high temporal resolution allowing perfusion parameters to be calculated. GBCAs are used for a broad range of clinical applications. However, despite the strong advantages of GBCAs, there is a number of drawbacks:

- Limitations in detection of low concentrations (i.e. detection limit).

- Non-linearity between signal intensity and contrast agent concentration

- The presence of a background signal from adjacent tissues

- Mono-spectral character of the signal, i.e. chemically different GBCAs cannot be spectrally discriminated

Emerging alternatives to GBCAs such as iron oxides, fluorine nanoparticles, CEST and hyperpolarized contrast agents have the potential to overcome some of these drawbacks. 
The lowest concentration of a GBCA detectable with MRI, the so-called detection limit, is in sub-millimolar (0.05-0.1 mM) range. Therefore, GBCAs are usually less suitable for targeted imaging of sparse molecular biomarkers, which usually express at far lower concentration levels. MRI of locally overexpressed biomarkers using targeted contrast agents, known as molecular MRI, is an emerging tool in preclinical and clinical research. For instance, it can be used to assess the level of angiogenesis, which is the growth of new blood vessels by targeting specific molecular addresses such as the integrin $a_{v} \beta_{3}$ or the CD13 protease. Angiogenesis accompanies physiologic growth processes such as the ovarian cycle and wound healing and is in adults usually at low level. In contrast, pathologic conditions such as cancer or cardiovascular ischemia, are associated with high levels of angiogenesis. Therefore, angiogenesis can be used as an early marker of tumor growth and malignancy, recovery after myocardial infarction and the development of atherosclerotic plaques. The expression levels of molecular biomarkers associated with angiogenesis however are usually lower than the detection limit of gadolinium with MRI. The detection limit of iron oxide contrast agents is, due to increased contrast per particle, much lower. When taking into account equal particle size, differences in relaxivity and the minimal detectable change in relaxivity, the effective detection limit is approximately a factor 12 higher ${ }^{[9]}$ for iron oxide contrast agents and therefore potentially better suited for molecular MRI than gadolinium. In cell labeling studies the detection of single cells loaded with iron oxide particles was demonstrated ${ }^{[10]}$.

Contrast enhancement by gadolinium is usually superimposed on the intrinsic signal variations of the human anatomy. Changes or spatial variations in contrast enhancement can therefore be difficult to distinguish from the anatomic background signal. This is particularly problematic for weak contrast enhancement when the contrast effect is less than the signal variations due to anatomy and noise. To improve the conspicuity of GBCAs, various MRI techniques exist that can suppress the background signal. For instance, for MR angiography, contrast in the vessel lumen can be enhanced by subtracting the contrast-enhanced images from those acquired prior to contrast administration. Other contrast-enhanced MR techniques make use of specific tissue (e.g. fat or muscle) signal suppression methods (e.g. radiofrequency pulses) to inhibit the signal of the background tissue. However, the level of suppression may need to be varied between patients and suppression pulses cannot always be applied due to limitations in the pulse sequence and scan time or other reasons. As an example, the contrast enhancement of blood can become as strong as the intrinsic signal of fat tissue, which makes it difficult to distinguish the blood vessels from surrounding fat. Fluorine MRI with a fluorinated $\left({ }^{19} \mathrm{~F}\right)$ contrast agent is an imaging technique, which may depict only the vessel lumen (i.e. without any background tissue signal) as tissue contains very little fluorine that is well below the detection limit. The fluorine MR image is usually used as an overlay on the anatomical, proton-based, image of the patient. The MR sensitivity of fluorine is almost as high as that of protons.

With GBCAs, signal contrast depends on relaxation times and its quantitative accuracy is influenced by the non-linear relationship between signal enhancement, concentration of the contrast agent and intrinsic magnetic properties of tissue and contrast agent. This makes quantification of the contrast agent concentration to some 
extent inaccurate and unreliable. Fluorine contrast agents have a linear relationship based on fluorine density (not relaxation) between signal intensity and concentration and could therefore improve quantitative MRI.

Another drawback of GBCAs is the fact that MR techniques are based on the detection of protons of water molecules that are magnetically affected by the paramagnetic gadolinium. As a consequence, the detection relies on an MR spectrum with only one peak (mono-spectral) and it is not possible to simultaneously use multiple contrasts for different molecular markers. Molecular MRI with multiple distinguishable targeted contrast agents could be advantageous in research and clinical diagnostics, for example in thrombosis. Contrast agents can be targeted to different biomarkers and contain gadolinium or fluorine as multi-spectral MR signal markers, which can be imaged separately at distinct resonance frequencies. Also different types of fluorine molecules can be used as multi-spectral MR signal markers due to their wide chemical and spectral range.

In this thesis, the applicability of iron oxide and fluorine based contrast agents will be explored to overcome certain drawbacks that appear in MRI of vascular pathology using GBCAs. 


\subsection{Biomarkers in vascular diseases}

For active targeting in molecular imaging, a contrast agent is targeted to a specific biomarker. Targeting is then performed by ligating a biomarker specific peptide or antibody to the contrast agent. A variety of biomarkers have been studied for molecular MRI, including specific biomarkers for vascular diseases. Previously, angiogenesis was already mentioned which can be targeted with the cyclic-NGR peptide. It has been shown that angiogenesis targeted contrast agents, with the NGR peptide, home to fast growing tumors which demand on developing new vessels. This is an example of a single target molecular MRI contrast agent. A multi-target contrast agent could be beneficial for assessing thrombosis. In thrombogenesis, thrombi are formed of thrombocytes and fibrin, and can obstruct the blood flow. In the acute stage of thrombus formation, the thrombus is stabilized covalently by cross-linking the fibrin to a stable network in the presence of a2-antiplasmine and factor XIII. During the stabilization, the thrombus is still treatable with fibrinolysis at an early stage of the cross-link process. By analyzing the availability of non-occupied "cross-link sides" on fibrin, the feasibility of a treatment can be assessed ${ }^{[11]}$. For this purpose, a multi-spectral dual targeted molecular MRI contrast agent could be beneficial. This contrast agent binds on two separate targets, for example, fibrin in general to visualize the thrombus and an a2antiplasmine based target to assess the treatability. To distinguish both targets, the ligated contrast agents have to be detectable with distinct spectral peaks. Gadolinium and fluorine can be used simultaneously as contrast agents, but two different fluorine molecules with different MR spectral peaks ${ }^{[12]}$ can also be distinguished in one image. A more detail description on the physiology of angiogenesis and fibrin clot formation is given in Box 1.1. 
Box 1.1 Physiology of angiogenesis and fibrin clot formation for molecular imaging

\section{Angiogenesis}

The process of angiogenesis, the sprouting of novel vessels from existing ones, occurs in an orderly series of steps. In tumors this process is trigged by hypoxia where various angiogenic factors are released, such as vascular endothelial growth factor (VEGF) and various cytokines. These angiogenic factors promote the growth of new blood capillaries through sprouting. Pericytes detach from the existing vessel wall and endothelial cells migrate and proliferate towards the tumor. During sprout formation, the endothelial cells release proteases to breakdown the extracellular matrix. Connection of neighboring sprouts occurs when endothelial cells of two sprouts form junctions and form a continuous lumen. The new blood vessel is normally matured by pericytes, however this step is often absent in fast growing tumors resulting in leaky blood vessels. Angiogenesis is associated with various biomarkers in the body; studies have shown that the aminopeptidase N/ CD13 transmembrane protease is expressed during angiogenesis in tumors ${ }^{[13]}$. The NGR (Asn-Gly-Arg) peptide motif is a CD13 ligand that has been used as a target for angiogenesis imaging and for therapeutically delivering cytotoxic drugs to the tumor vasculature ${ }^{[14]}$.

\section{Fibrin clot formation}

The forming of a fibrin network during clot formation is the last major step in the blood coagulation cascade and part of the common pathway of coagulation. Factor $\mathrm{X}$ is activated to $\mathrm{Xa}$, either by the intrinsic pathway through factor IXa and VIIla, or by the extrinsic pathway through factor VIIa and tissue factor. Factor Xa activates circulating prothrombin into thrombin, which converts circulating fibrinogen into fibrin. Fibrin is an insoluble protein, because during fibrin clot formation it polymerizes to long strands to which activated platelets can bind. The last step is covalent cross-linking of fibrin by factor XIIIa, while at the same time a2-antiplasmine is coupled to fibrin by factor XIIIa ${ }^{[15,16]}$. In hemostatic blood clots, the cross-linked fibrin network forms a mesh stabilizing the platelet plug and stabilizes the clot. The cross-linking process of a2-antiplasmine to fibrin requires factor XIIla and only occurs in fresh thrombi ${ }^{[17]}$. Therefore targeted contrast media with a2-antiplasmine-based ligands enable specific visualization of early thrombus formation ${ }^{[18]}$. 


\subsection{Contrast mechanisms of MRI contrast agents}

This paragraph introduces the concepts of contrast mechanisms of MR imaging. It will explain spin-density, $T_{1}$ and $T_{2}$ contrast mechanisms and susceptibility induced contrast. Throughout this paragraph there will be referred to boxes which provide the basic physics of these effects.

Tissue contrast in MR images is based on the signal intensity in a certain molecular environment and depends on three tissue properties; spin density and $T_{1}$ and $T_{2}$ relaxation times. The spin density, i.e. proton density (PD) for ${ }^{1} \mathrm{H}$ MRI and ${ }^{19} \mathrm{~F}$ density for fluorine MRI, represents the number of nuclei in a voxel of interest. $T_{1}$ is the longitudinal or spin-lattice relaxation time and $T_{2}$ is the transverse or spin-spin relaxation time. In MRI measurements, specific weighing of spin density, $T_{1}$ or $T_{2}$ can be chosen by adjusting the repetition $\left(T_{r}\right)$ or echo $\left(T_{e}\right)$ time in the MR pulse sequence (Box 1.2).

\section{Spin density, $\mathrm{T}_{1}$ and $\mathrm{T}_{2}$ contrasts}

Contrast agents are used to locally affect the spin density, $T_{1}$ or $T_{2}$ relaxation time of tissue and to enhance the contrast between diseased and healthy tissue. Spin density contrast is rarely used in clinical examinations with or without GBCAs, however this contrast mechanism is important for fluorine MRI. Fluorine is in vivo only found in bone and teeth, where it is immobile and not measurable with clinical MR systems. The absence of an endogenous fluorine background in the human body greatly reduces the background signal and thus increases sensitivity for signal detection. The fluorine density in a voxel is directly and linearly related to the measured signal intensity.

The different relaxation time shortening effects of contrast agents has led to the general classification of $T_{1}$ or $T_{2}$ contrast agents, because they mainly affect the $T_{1}$ or

Box 1.2 - Signal intensity by spin density, $T_{1}$ or $T_{2}$.

The signal intensity, $S I$, is exponentially dependent on the $T_{1}$ and $T_{2}$ relaxation time as shown in equation 1.1. In this equation, which is for instance applicable for spin echo methods, $S_{0}$ represents the spin density, i.e. the proton or fluorine density in a voxel.

$$
S I=S_{0}\left(1-e^{-\frac{T_{r}}{T_{1}}}\right) e^{-\frac{T_{e}}{T_{2}}}
$$

The strength of spin density, $T_{1}$ or $T_{2}$ weighing for the image contrast can be chosen by adjusting the $T_{r}$ and $T_{e}$ in an MR sequence (Table 1.1).

Table 1.1 Relaxation contrast dependencies on contrast parameters $T_{e}$ and $T_{r}$.

\begin{tabular}{lll}
\hline & \multicolumn{1}{c}{$T_{e}$} & \multicolumn{1}{c}{$T_{r}$} \\
\hline$T_{1}$ weighing & Short & In order of tissue $T_{1}$ \\
$T_{2}$ weighing & In order of tissue $T_{2}$ & Long \\
Spin density weighing & Short & Long \\
\hline
\end{tabular}


Table 1.2 - Main properties of $T_{1}$ and $T_{2}$ contrast agents.

\begin{tabular}{lll}
\hline & \multicolumn{1}{c}{$T_{1}$ contrast agents } & \multicolumn{1}{c}{$T_{2}$ contrast agents } \\
\hline Magnetic material & Gadolinium & Iron Oxide \\
Magnetization & Paramagnetic & Superparamagnetic \\
Relaxation time effect & Shortening $T_{1}$ & Shortening $T_{2}$ \\
Contrast mechanism & Dipole-dipole interaction & Local disturbance of the \\
& between nuclear spins & static magnetic field, \\
& and the fluctuating local & resulting in an locally \\
& magnetic field[21] & increased dephasing of spins \\
Size & Molecular $(<1 \mathrm{~nm})$ & Nanoparticle $(50 \mathrm{~nm}-1 \mu \mathrm{m})$ \\
\hline
\end{tabular}

$T_{2}$ relaxation time of water. It is important to note that these $T_{1}$ and $T_{2}$ contrast agents are measured indirectly: not the density of the contrast agent is measured, but rather the shortening of the corresponding relaxation time of the surrounding water. The changes in relaxation times are usually expressed as relaxation rates, i.e. $R_{1}=1 / T_{1}$ or $R_{2}=1 / T_{2}$, where differences in relaxation rate leads to a signal change (contrast). The ability of a contrast agent to increase the relaxation rate is proportional, and for low concentrations linear, related to the contrast agent's relaxivity and concentration (Box 1.3). Contrast agents with a relatively high relaxivity have an increased relaxation rate and therefore the relaxivity can be considered as the efficiency of a contrast agent. The relaxivity of GBCAs is dependent on the static magnetic field and its chemical surrounding ${ }^{[19,20]}$. For iron oxides, or $T_{2}$ contrast agents, the relaxivity depends on the magnetic moment of the iron oxide crystal, its size and coating and the distribution and location of the agent in the body. The classification of $T_{1}$ and $T_{2}$ contrast agents are mainly defined by the magnetization of a contrast agent, where paramagnetic contrast agents are classified as $T_{1}$ and superparamagnetic contrast agents as $T_{2}$. The main properties of $T_{1}$ and $T_{2}$ contrast agents are summarized in Table 1.2.

Box 1.3 Relation between relaxation rate, relaxivity and concentration

Contrast enhancement is the result of a change in relaxation rate $R_{1}\left(\mathrm{~s}^{-1}\right)$ and/or $R_{2}$ $\left(\mathrm{s}^{-1}\right)$ with respect to the intrinsic relaxation rate of the tissue $R_{10}\left(\mathrm{~s}^{-1}\right)$ and/or $R_{20}\left(\mathrm{~s}^{-1}\right)$. This change is proportional with the concentration $c(\mathrm{mM})$ and the relaxivity $r_{1}$ or $r_{2}$ $\left(\mathrm{mM}^{-1} \mathrm{~s}^{-1}\right)$ of the contrast agent as given in the following equations.

$$
\begin{array}{ll}
\text { For } T_{1} & R_{1}=\frac{1}{T_{1}}=R_{10}+r_{1} c \\
\text { For } T_{2} & R_{2}=\frac{1}{T_{2}}=R_{20}+r_{2} c
\end{array}
$$

Paramagnetic $T_{1}$ contrast agents have, in general, a high $r_{1}$ relaxivity, whereas superparamagnetic contrast agents have a high $r_{2}$ relaxivity. It should be noticed that equation 1.2 is valid for contrast agents in a water solution and that a linear relationship is only valid at relatively low concentrations. At higher concentrations, the $T_{2}$ effect of GBCAs becomes noticeable resulting in weaker increases or even decreases in contrast enhancement. 
Contrast enhancement for $T_{1}$ contrast agents results in a signal hyperintensity on $T_{1}$ weighted images whereas for $T_{2}$ contrast agents a signal hypointensity is depicted in $T_{2}$ weighted images. Therefore $T_{1}$ agents are also referred to as positive contrast agents, while $T_{2}$ agents are considered negative contrast agents. For low concentrations, the contrast effect is approximately linear, but for higher concentrations the contrast enhancement depends also on other relaxation effects. As a result, $T_{2}$ effects (signal voids) become noticeable in a $T_{1}$ weighted image. The same effect applies for $T_{2}$ contrast agents where $T_{2}^{*}$ effects (signal voids and susceptibility artifacts) appear at higher concentrations. All contrast effects, including the contrast effect of a fluorine spin-density contrast agent, are shown in Figure 1.1.

\section{$T_{2}^{*}$ contrast and magnetic susceptibility}

MR imaging is based on the properties of magnetic nuclear spins that reside in a strong and homogeneous magnetic field, which leads to phase coherencies and equal resonance frequencies of many spins (magnetic moments) to form a detectable magnetic signal. The resonance or Larmor frequency of a nuclear spin depends on the strength of the magnetic field. Inhomogeneities in this magnetic field can disturb this phase coherence and induce resonance frequency offsets in spins. Such magnetic field inhomogeneities can occur at intersections between different tissues or tissueair interfaces. The magnetic susceptibility of these tissues or materials is different, resulting in a disturbance of the static magnetic field. Another source of magnetic field inhomogeneities is contrast agents that strongly affect the magnetization, for instance, superparamagnetic $\left(T_{2}\right)$ particles.

Spin dephasing, caused by inhomogeneities in the magnetic field, results in signal loss and imaging artifacts. Spin echo pulse sequences can compensate for the effects of local static field inhomogeneities and the pertaining spin dephasing. For $T_{2}$ contrast agents the native $T_{2}$ contrast enhancement can therefore be measured with a spin-echo pulse sequence, because the $180^{\circ}$ refocusing pulse corrects for the spin dephasing due to (static) field inhomogeneities ${ }^{[22]}$. Gradient echo pulse sequences do not compensate for the spin dephasing and the disturbance of the magnetic field induced by the contrast agent is not compensated. The transversal relaxation in gradient echo imaging is therefore referred to as $T_{2}{ }_{2}^{*}$ relaxation and consists of two parts, the $T_{2}$ relaxation (from spin-spin interaction) and additional dephasing due to (static) field inhomogeneities. Due to this dephasing, a gradient echo pulse sequence is therefore much more sensitive for $T_{2}$ contrast agents compared to spin-echo pulse sequences.

The $r_{2}{ }^{*}$ relaxivity of superparamagnetic agents can create a much stronger contrast compared to gadolinium particles. Iron oxide particles are therefore more suitable for molecular MRI to detect low concentrations of molecular biomarkers. Within the voxel, this results in strong spin dephasing and a strong (negative) contrast enhancement, because the contrast agent causes hypointensities in the MR image. The magnetic disturbance that changes the resonance frequency result in off-resonance spins and is not limited to the voxel but extends to the voxel's perimeter (Box 1.4). The off-resonance spins will show as hypointensities in the MR image. Recently, special 
positive contrast imaging techniques were developed that rely on the effect of dephasing or off-resonances spins of superparamagnetic contrast agents. These imaging techniques are described in more detail in chapter 2.

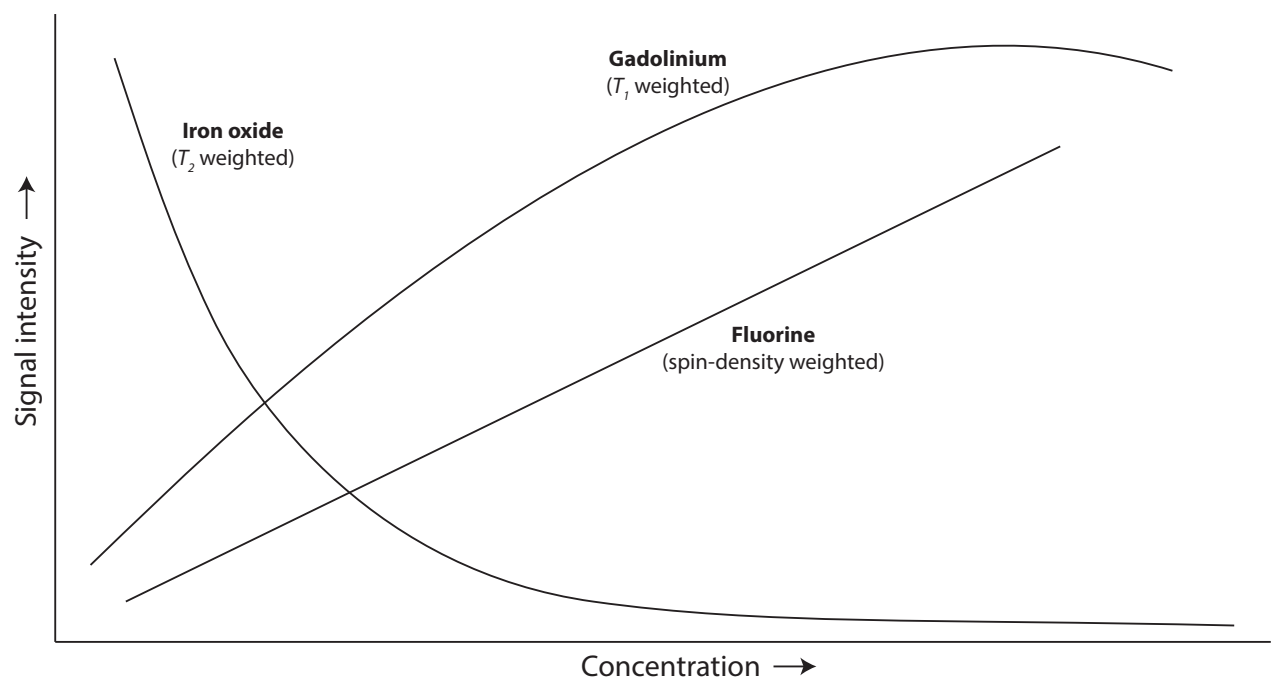

Figure 1.1 - The relation between the contrast agent concentration and the signal intensity for $T_{1}, T_{2}$ and fluorine contrast agents (depicted independently on an arbitrary scale). Gadolinium, a $T$, contrast agent, shows a linear (positive) relation for low concentrations on a $T_{1}$ weighted MR image. For higher concentrations, this relation is non-linear due to the contribution of the $T_{2}$ contrast effect. Iron oxide, a $T_{2}$ contrast agent, has a (negative) linear relation for low concentrations on $T_{2}$ weighted images. However, at higher concentrations $T_{2}$ * effects induce signal voids and susceptibility artifacts. Fluorine contrast agents show a linear relation between the concentration and the signal intensity on spin-density weighted images for a wide range of fluorine (spin density) concentrations.

Box 1.4 Off-resonance and magnetic field perturbations of $T_{2}$ contrast agents.

For a gradient echo sequence, the change in magnetic field, $\Delta B$, due to changes in susceptibility can be used to derive a phase change, $\Delta \phi$, of the magnetization as function of echo time (Equation 1.3). A change in local magnetic field means also a change in precession frequency (i.e. offset in resonance frequency). The precession frequency is directly related to the absolute value of the local magnetic field.

$$
\begin{gathered}
\Delta \phi\left(T_{e}\right)=-\gamma \Delta B T_{e} \\
f=\frac{\gamma}{2 \pi}\left(B_{0}+\Delta B\right)
\end{gathered}
$$

Where $\gamma / 2 \pi$ is the gyromagnetic ratio $(42.576 \mathrm{MHz} / \mathrm{T}), B_{0}$ the static magnetic field in Tesla, and $f$ the Larmor frequency in Hertz. Superparamagnetic contrast agents 
will affect the Larmor frequency and phase in the voxel and its surrounding voxels in a distant dependent way.

\section{In vivo field perturbation}

The magnetic susceptibility, $\chi$, is the degree in which a material can be magnetized by an external magnetic field and is a dimensionless material property. For paramagnetic materials $\chi>0$ and for diamagnetic materials $\chi<0$. Magnetic susceptibility of human tissue is very small and is diamagnetic. Most tissues in the human body contain water, which is diamagnetic and has a susceptibility $\chi_{\text {water }}$ $\simeq-9 \cdot 10^{-6}$. The susceptibility for paramagnetic and superparamagnetic contrast agents is much higher and lies in the order of $10^{-5}$ and $10^{-3}$, respectively. A change in susceptibility at boundaries or interfaces of materials with different magnetic susceptibilities gives rise to a change in magnetic field, not only in the proximity of contrast agents but also at tissue-tissue or tissue-air interfaces. The change in magnetic field, as a result of the change in magnetic susceptibility, can be calculated and simulated.

The in vivo field perturbations for a small tumor or thrombus labeled with superparamagnetic contrast agents can be calculated when we assume its geometry as a sphere like volume. Equations 1.5a and 1.5b describe this magnetic field for a sphere filled with a contrast agent.

$$
\begin{gathered}
\Delta B_{z}=\frac{2 \chi}{3+\chi} B_{0} ; r<a \\
\Delta B_{z}=B_{0} \frac{\chi}{3+\chi}\left(\frac{a}{r}\right)^{3}\left(3 \cos ^{2} \varphi-1\right) ; r>a
\end{gathered}
$$

The (static) external magnetic field is assumed to be along the $z$ axis, i.e. $\overrightarrow{B_{0}}=B_{0} \hat{z}$ .These equations use a polar coordinate system with $r$ the radius, $\varphi$ the angle between the field $B_{0}$ and $r ; a$ is the radius of the sphere as depicted in Figure 1.2.

Another common situation is a blood vessel containing contrast agent. This blood vessel can be represented as a cylindrical body with infinite length. The axis of the cylinder has one degree of freedom with respect to the external field $B_{0}$. In this case the cylinder is positioned perpendicular to $B_{0}$ and for that configuration the change in magnetic field inside and outside the cylinder can be calculated with equations $1.6 a$ and $1.6 b{ }^{[22]}$. The same polar coordinate system is applied as for the sphere.

$$
\begin{gathered}
\Delta B_{z}=B_{0} \frac{\chi / 2}{1+\chi / 2} ; r<a \\
\Delta B_{z}=B_{0} \frac{\chi / 2}{1+\chi / 2}\left(\frac{a}{r}\right)^{2} \cos 2 \varphi ; r>a
\end{gathered}
$$

The 2D spatial distortion of $\Delta B_{z}$ is calculated and shown in Figure 1.3. 
This calculation describes the change in magnetic field in the surrounding of the two objects. Two positive and two negatives lobes describe this field which has its strongest intensity at the direct vicinity of the object. This calculation also shows the extent of the field distortion, which can be multiple times the object size. The change in magnetic field represents the change in phase in the MR image (equation 1.3), however it does not represent the intensity values of the magnitude image, which is normally used to represent the anatomy. The previously calculated field perturbation is used as input for the MRI simulator tool JEMRIS [23]. The magnitude and phase image results of this simulation are shown in Figure 1.4. for both objects. The magnitude and phase images shows a large similarity with the calculations of $\Delta B_{z}$; all the images show the characteristic four lobes and the distortion is noticeable in the vicinity of the objects.

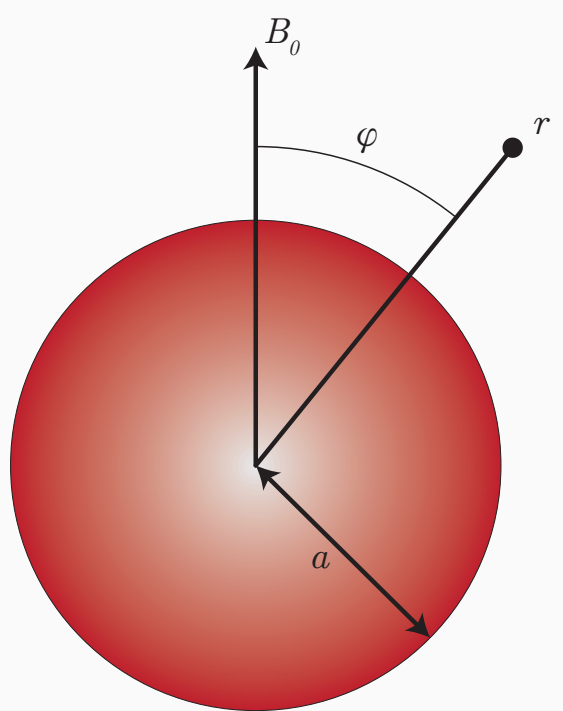

Figure 1.2 - The space surrounding a magnetized sphere or cylinder is described by a polar coordinate system from a centralized point with distance $r$ and an angle $\varphi$ with respect to the external field. The sphere or cylinder has a radius $a$. In case of a cylinder, the axis of the cylinder is positioned perpendicular to the external field. 

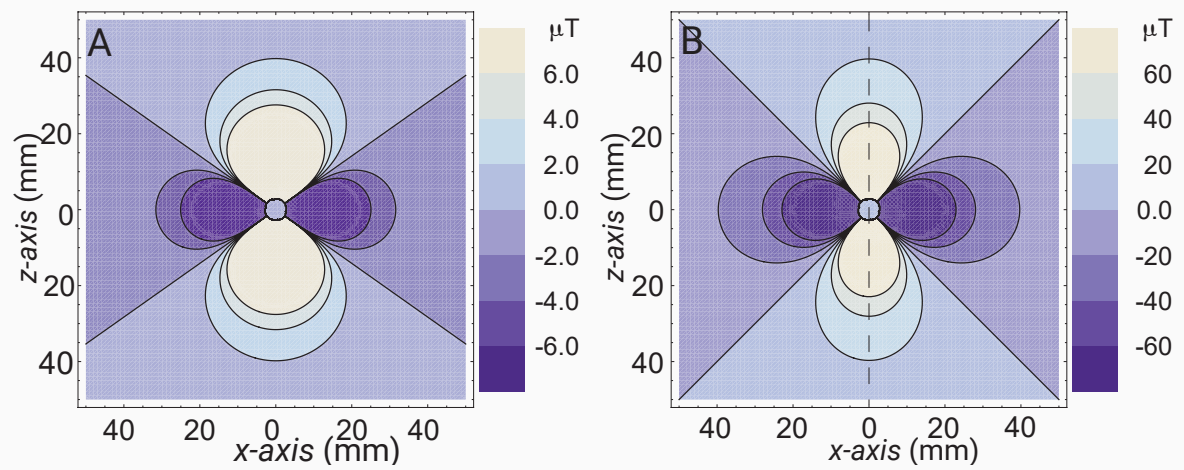

Figure 1.3 - Simulation of the spatial distortion, $\Delta B$, of the external field for a sphere (A) and cylinder (B). For both geometries: radius $a=3 \mathrm{~mm}$, susceptibility $\chi=1 \cdot 10-3$ and the external field $B_{0}=7$ Tesla. $\Delta B$ is only calculated outside the cylinder, the color value inside the cylinder is arbitrary.

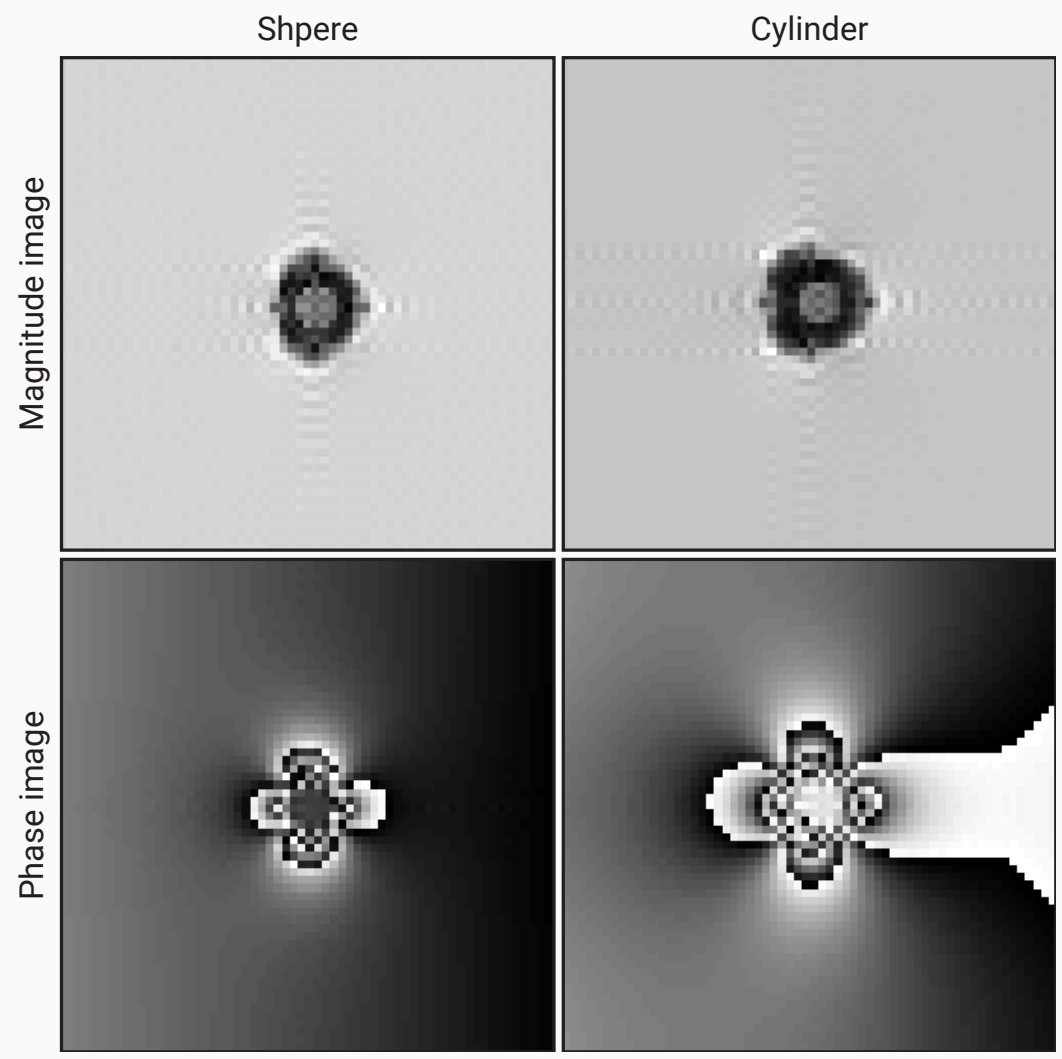

Figure 1.4 MRI simulations of a sphere and a cylinder with a susceptibility difference between the inside and outside region. For these simulations the geometrical shape, $T_{1}$, $T_{2}$, and the $\Delta B$ are given as input parameters. After the simulation a magnitude and phase image is returned. Under the given conditions the phase images of both objects in the simulation returned the so-called phase wraps. 


\subsection{Thesis objectives and outline}

The main goal of this thesis is to assess the applicability of iron oxide and fluorine based contrast agents, and their associated imaging techniques, to overcome specific drawbacks of GBCAs in certain preclinical applications.

In chapter 2, a preclinical study investigates the use of a targeted iron oxide contrast agent for the visualization of angiogenesis. Iron oxide contrast agents have a low detection limit, which makes it ideal for molecular MRI. With conventional MRI, iron oxide contrast agents are usually depicted as hypo intense areas. In this chapter various imaging techniques are compared to depict the angiogenesis targeted iron oxide contrast agent as positive contrast.

Inducing arterial thrombi in animal models with Ferric Chloride is widely used. However, for MRI research, the use of this model can induce large susceptibility artifacts. The preclinical study in chapter $\mathbf{3}$ shows an alternative solution for inducing thrombi in the acute thrombus mouse model without the risk of MRI artifacts, which makes it a more reliable model for molecular MRI.

A comprehensive overview of fluorine MRI and MR spectroscopy is presented in chapter 4. It describes the different types of fluorine contrast agents and imaging techniques for fluorine MR that are available. This overview also presents (emerging) clinical applications, for instance drug tracking, lung ventilation imaging and molecular MRI, where fluorine contrast agents are more beneficial compared to GBCAs.

Chapter 5 investigates the visualization of thrombi with targeted fluorine loaded nanoparticles emulsions. In this preclinical study we have explored the utility of novel fluorine loaded polymer nanoparticles labeled with the fibrin specific peptide A14. This targeted fluorine contrast agent could be a candidate for multi-spectral molecular MRI.

Most fluorine contrast agents usually suffer from a long $T$, relaxation time, which increases the acquisition time for $3 \mathrm{D}$ acquisition dramatically. An accelerated isotropic 3D fluorine imaging technique is presented in chapter 6 .

Chapter 7 provides a general discussion on nanoparticle contrast agents for MRI and the future perspectives towards clinical translation of iron oxide or fluorine contrast agents are discussed. 


\section{References}

1. Hu, H.H. and H.E. Kan, Quantitative proton MR techniques for measuring fat. NMR Biomed, 2013. 26(12): p. 1609-29.

2. Rossi, A. and R. Biancheri, Magnetic resonance spectroscopy in metabolic disorders. Neuroimaging Clin N Am, 2013. 23(3): p. 425-48.

3. Wheaton, A.J. and M. Miyazaki, Non-contrast enhanced MR angiography: physical principles. J Magn Reson Imaging, 2012. 36(2): p. 286-304.

4. Battal, B., et al., Cerebrospinal fluid flow imaging by using phase-contrast $\mathrm{MR}$ technique. $\mathrm{Br}$ J Radiol, 2011. 84(1004): p. 758-65.

5. Tournier, J.D., S. Mori, and A. Leemans, Diffusion tensor imaging and beyond. Magn Reson Med, 2011. 65(6): p. 1532-56.

6. Aime, S. and P. Caravan, Biodistribution of gadolinium-based contrast agents, including gadolinium deposition. J Magn Reson Imaging, 2009. 30(6): p. 1259-67.

7. Prince, M.R., et al., Breath-hold gadolinium-enhanced MR angiography of the abdominal aorta and its major branches. Radiology, 1995. 197(3): p. 785-92.

8. Choyke, P.L., A.J. Dwyer, and M.V. Knopp, Functional tumor imaging with dynamic contrastenhanced magnetic resonance imaging. J Magn Reson Imaging, 2003. 17(5): p. 509-20.

9. Gore, J.C., et al., Molecular imaging without radiopharmaceuticals? J Nucl Med, 2009. 50(6): p. 999-1007.

10. Bulte, J.W. and D.L. Kraitchman, Iron oxide MR contrast agents for molecular and cellular imaging. NMR Biomed, 2004. 17(7): p. 484-99.

11. Phinikaridou, A., et al., Molecular MRI of atherosclerosis. Molecules, 2013. 18(11): p. 14042-69.

12. Jacoby, C., et al., Probing different perfluorocarbons for in vivo inflammation imaging by $F$ MRI: image reconstruction, biological half-lives and sensitivity. NMR Biomed, 2013.

13. Pasqualini, R., et al., Aminopeptidase $\mathrm{N}$ is a receptor for tumor-homing peptides and a target for inhibiting angiogenesis. Cancer Res, 2000. 60(3): p. 722-7.

14. Bartz, R., et al., Effective siRNA delivery and target mRNA degradation using an amphipathic peptide to facilitate pH-dependent endosomal escape. Biochem J, 2011. 435(2): p. 475-87.

15. Abdul, S., et al., Natural heterogeneity of alpha2-antiplasmin: functional and clinical consequences. Blood, 2016. 127(5): p. 538-45.

16. Muszbek, L., V.C. Yee, and Z. Hevessy, Blood coagulation factor XIII: structure and function. Thromb Res, 1999. 94(5): p. 271-305.

17. Robinson, B.R., A.K. Houng, and G.L. Reed, Catalytic life of activated factor XIII in thrombi. Implications for fibrinolytic resistance and thrombus aging. Circulation, 2000. 102(10): p. 1151-7.

18. Miserus, R.J., et al., Molecular MRI of early thrombus formation using a bimodal alpha2antiplasmin-based contrast agent. JACC Cardiovasc Imaging, 2009. 2(8): p. 987-96.

19. Stanisz, G.J. and R.M. Henkelman, Gd-DTPA relaxivity depends on macromolecular content. Magn Reson Med, 2000. 44(5): p. 665-7. 
20. Landis, C.S., et al., Determination of the MRI contrast agent concentration time course in vivo following bolus injection: effect of equilibrium transcytolemmal water exchange. Magn Reson Med, 2000. 44(4): p. 563-74.

21. Toth, E., L. Helm, and A.E. Merbach, Relaxivity of Gadolinium(III) Complexes: Theory and Mechanism, in The Chemistry of Contrast Agents in Medical Magnetic Resonance Imaging, A.E. Merbach and E. Toth, Editors. 2001, John Wiley \& Sons, Ltd. p. 44-119.

22. Haacke, E.M., Magnetic resonance imaging : physical principles and sequence design. 1999, New York: Wiley. xxvii, 914 p.

23. Stocker, T., et al., High-performance computing MRI simulations. Magn Reson Med, 2010. 64(1): p. 186-93. 
Purpose Superparamagnetic iron oxide particles (SPIOs) are promising contrast agents for molecular MRI. To improve the in vivo detection of iron-based contrast media, positive contrast imaging techniques have been developed. Here, the efficacy of two positive contrast techniques, White Marker and Susceptibility Gradient Mapping (SGM), were evaluated for molecular MRI of tumor angiogenesis and compared to conventional negative contrast gradient echo (GE) imaging.

Methods In vitro: cylindrical phantoms containing varying iron oxide concentrations were used to measure the response of positive contrast techniques. In vivo: tumor bearing mice were used as a model for tumor angiogenesis. Mice were injected with unlabeled SPIOs $(n=5)$ or SPIOs labeled with cyclic NGR peptide (cNGR) $(n=5)$, which homes specifically to angiogenic microvessels. Pre- and post-contrast GE and White Marker images were acquired. Subsequently, SGM images and $R_{2}{ }^{*}$ maps were calculated. For image analysis, the contrast-to-noise ratio (CNR) and the percentage of enhanced voxels (EVs) in the tumor rim and core were calculated.

Results In vitro: the linear increases in MRI signal response for increasing iron oxide concentration was much stronger for SGM than White Marker. In vivo: the CNR of GE, White Marker and SGM imaging was 5.7, 1.2 and 6.2, respectively, with equal acquisition times. Significant differences in the percentage of $E V s$ between the tumor rim and core were found using $R_{2}{ }^{*}$ mapping, GE and SGM $(P<0.05)$. The two contrast agents had significantly different percentages of EVs by $R_{2}{ }^{*}$ mapping and SGM in the rim $(P<0.001)$.

Conclusion The in vivo efficacy of White Marker and SGM was evaluated for molecular MRI relative to GE imaging and $R_{2}{ }^{*}$ mapping. Only SGM, and not White Marker, can be used to transfer the negative contrast from targeted SPIOs in a positive contrast signal without loss of CNR. 


\subsection{Introduction}

Molecular imaging involves the detection of sparse biomarkers and requires high sensitivity of the contrast agent and imaging technique ${ }^{[1,2]}$. MRI is a highly desirable modality for molecular imaging as it provides high spatial resolution and excellent soft tissue contrast. However, the inherently low sensitivity of MRI for the detection of contrast agents makes imaging of sparse biomarkers of diseases more difficult compared with nuclear or optical techniques. To obtain sufficient $T_{1}$-weighted image contrast, relatively large particles (e.g. polymers, liposomes, micelles) are usually administered, carrying a high payload of gadolinium ${ }^{[3-5]}$. Alternatively, iron oxide particles can be applied to generate $T_{2}$ and, in particular, $T_{2}{ }^{*}$-weighted contrast. These susceptibility contrast agents are known for their good sensitivity at low particle concentrations ${ }^{[2]}$. An example of molecular MRI of angiogenesis using a targeted ultra small superparamagnetic iron oxide (USPIO)-based contrast agent was provided by Zhang et al ${ }^{[6]}$. As the accumulation of iron oxides in tissue generally results in reduced $T_{2}^{*}$ relaxation times, the signal decrease induced by the ironparticles (i.e. negative contrast) can be difficult to discern from intrinsically hypointense or heterogeneous background tissue, especially at higher field strengths.

To overcome this issue, several positive contrast techniques have been developed which generate hyperintensities in the vicinity of iron oxide nanoparticles. These techniques can be divided into three groups: gradient sensitive techniques ${ }^{[7,8]}$, frequency sensitive techniques ${ }^{[9-11]}$ and post-processing techniques ${ }^{[12,13]}$. The present study evaluates two positive contrast techniques, a gradient sensitive technique, White Marker ${ }^{[7]}$ and a post-processing technique, Susceptibility Gradient Mapping (SGM) ${ }^{[12]}$. The theoretical background of White Marker and SGM is summarized in Figure 2.1.

The main objective of this study was to compare the two positive contrast techniques with traditional gradient echo (GE) imaging for molecular MRI of tumor angiogenesis using targeting SPIOs. The feasibility and in vivo application of positive contrast MRI has been demonstrated previously $[7,12,14,15]$. However, so far it remained unclear whether this can be used for molecular imaging and how the contrast effect compares to negative contrast imaging. The current study presents the applicability of White Marker and SGM to depict uptake in angiogenesis exhibiting tumors using targeted iron oxide nanoparticles. Both techniques were first optimized and compared in phantoms and then evaluated in tumorbearing mice using cyclic NGR (CNGR) tripeptide-labeled iron oxide particles that home to activated endothelial cells of angiogenic blood vessels. The cNGR peptide has a high affinity for CD13, which is a well-known angiogenic marker ${ }^{[16-21]}$. This peptide has already successfully been used in experimental molecular MRI studies of angiogenesis in tumors and in the heart ${ }^{[22,23]}$. 
A
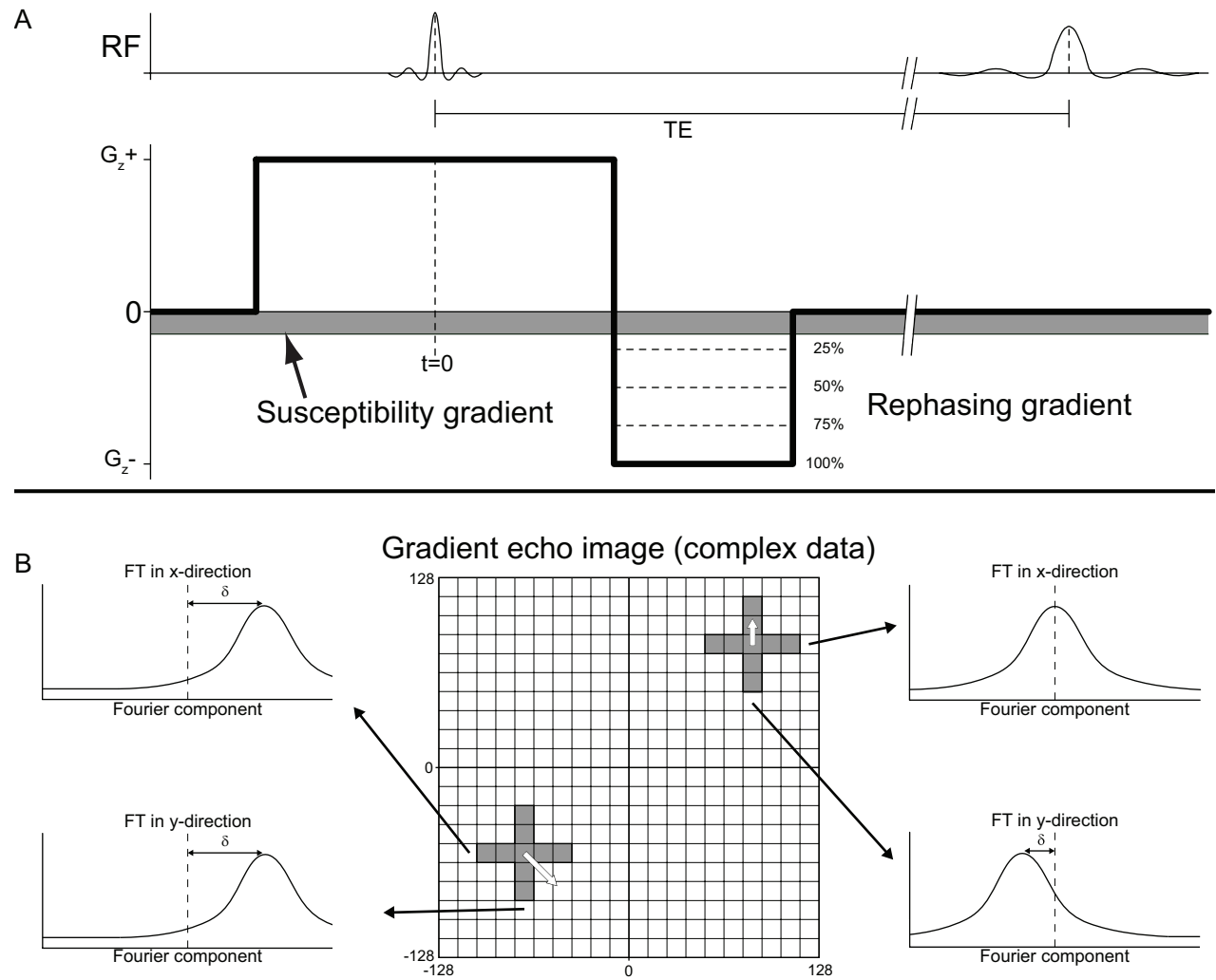

Figure 2.1 - Panel A shows the slice selection gradient (Gz), the susceptibility gradient and the RF signal (RF) of the White Marker ${ }^{[7]}$ sequence. For conventional gradient echo (GE) imaging, the dephasing of spins in the selected slice is compensated by the rephrasing gradient of equal strength and opposite direction. The presence of iron-oxide particles will induce field inhomogeneities, which is represented by the susceptibility gradient (gray). Due to this susceptibility gradient, the dephasing of the spins will be altered and will not be completely rephrased by the original slice selection gradient, which will lead to signal loss. For White Marker, the strength of the rephasing gradient can be adjusted (0-100\%) to compensate for the additional dephasing effect of the iron-oxide induced susceptibility gradient. The correct rephasing depends on the strength of the rephasing gradient, the susceptibility gradient, and the echo time.

Panel B shows the principle of SGM ${ }^{[12]}$. The SGM image is calculated from the complex pixel values of a GE image. A sliding window of 5 pixels samples the complex data of the GE image. Subsequently, these data samples are Fourier transformed to a short-term k-space. The susceptibility gradient (white arrow), is determined from the shift $\delta$ of the maximum in the Fourier spectrum relative to the spatial frequency $\mathrm{k}=0$. This shift is proportional to the strength of the susceptibility gradient and was assigned to the center position of the sliding window in the SGM image. With SGM, it is possible to slide a window in two or three directions to obtaina 2D or 3D susceptibility vector, respectively. 


\subsection{Methods and Materials}

\section{Phantom MRI experiments}

To test and optimize the different MRI acquisition techniques, two phantoms were used: 1) a single iron oxide cylinder phantom to optimize acquisition and imaging techniques, and 2) a phantom with multiple iron oxide cylinders to determine the signal response of positive contrast techniques on different iron oxide concentrations.

For the first phantom, the single cylinder phantom, a cylindrical tube (diameter 30 $\mathrm{mm}$; length $70 \mathrm{~mm}$ ) was filled with $2 \%$ agarose gel. The center of this phantom contained a $3 \mathrm{~mm}$ cylindrical core filled with $2 \%$ agarose gel containing $0.15 \mathrm{mmol}$ $\mathrm{Fe} / \mathrm{L}$ (Resovist $0.5 \mathrm{~mol}$ Fe/L, Schering). Imaging was performed on a $7.0 \mathrm{~T}$ horizontal bore MRI system (Bruker Avance II Biospec 70/30 USR, Ettlingen, Germany) equipped with a BGA12 mini imaging gradient system (maximum gradient strength $400 \mathrm{mTm}^{-1}$; slew rate, $4000 \mathrm{Tm}^{-1} \mathrm{~s}^{-1}$ ) and a quadrature volume resonator (inner diameter $82 \mathrm{~mm}$ ). The cylindrical axis of the phantom was placed vertically in the (horizontal) magnet bore and perpendicular to the magnetic field $B_{0}$. In this orientation, a paramagnetic cylinder will induce susceptibility gradients perpendicular to the cylinder and the susceptibility gradient parallel to the cylinder is minimized. This phantom, with a well-known susceptibility geometry, was used to optimize the imaging techniques. For image acquisition, a series of conventional GE sequences (TE=3.5, 7, 10, 15, 25 $\mathrm{ms}, \mathrm{TR}=750 \mathrm{~ms}$, and $\mathrm{FA}=40^{\circ}$ ) and a series of White Marker sequences (TE=5 ms, $\mathrm{TR}=750 \mathrm{~ms}, \mathrm{FA}=40^{\circ}$, rephasing 100, 75, 50, 25, $0 \%$ ) were applied. The White Marker sequence is a GE sequence with an altered rephasing gradient in the slice selection (Figure 2.1 Panel A); 100\% rephasing represents a complete rephasing and is therefore equal to a conventional GE acquisition. 25\% rephasing is a quarter of the strength of the rephasing gradient of a GE acquisition. For both the White Marker and the GE sequences, five 1-mm thick axial slices with a gap of $1 \mathrm{~mm}$ were acquired. The acquired matrix was $256 \times 256$ and the field of view (FOV) was $30 \times 30 \mathrm{~mm}$, resulting in $0.12 \times 0.12 \mathrm{~mm}^{2}$ sized voxels. White Marker depicts only positive contrast in the slice direction. Therefore, axial slices (i.e. short-axis sections) are not useful and coronal slices (i.e. long-axis sections) were applied, due to the minimal susceptibility gradient parallel to the cylinder in this orientation.

For the second phantom, the multiple cylinder phantom, a $30 \mathrm{~mm}$ cylindrical tube with 9 vertical cylinders, $1 \mathrm{~mm}$ in diameter and $10 \mathrm{~mm}$ in height, was designed (Figure 2.3 A and B). The cylinders were filled with iron concentrations of $0.0,0.1,0.2,0.3,0.6$, $0.7,0.8,0.9$ and $1.0 \mathrm{mmol} F e / L$, diluted in saline buffer. For gradient echo imaging, the same setup up was used as for the first phantom experiment. A single $1 \mathrm{~mm}$ axial (short-axis) slice was acquired with a conventional GE sequences (TE=3.5, 7, $15 \mathrm{~ms}$, TR=750 ms, and $\mathrm{FA}=40^{\circ}$ ). For White Marker 100\%, 50\% and 25\% rephasing was applied. For each rephasing value two orthogonal long-axis (one parallel and one perpendicular to $B_{0}$ ) acquisitions were made. Both acquisitions had a matrix of $256 \mathrm{x}$ 256,64 slices and a voxel size of $0.2 \times 0.2 \times 0.8 \mathrm{~mm}^{3}$. The image data of acquisition were 
interpolated to obtain one reformatted image, i.e. an axial (short-axis) image (pixel size, $0.2 \times 0.2 \mathrm{~mm}^{2}$ ) as used for the gradient echo acquisition.

\section{Contrast agent}

Streptavidin-coated SPIOs $\left(\mu \mathrm{MACS}^{\mathrm{T}}\right.$ ) were obtained from Miltenyi Biotec (Bergisch Gladbach, Germany) ${ }^{[24]}$. The absolute iron concentration in the SPIO solution was 0.28 $\mathrm{mg} / \mathrm{ml}$, as measured using Inductively Coupled Plasma Mass Spectrometry, which is equivalent to an iron concentration of $5 \mathrm{mM}$. To measure $r_{1}, r_{2}$ and $r_{2}^{*}$ relaxivities, SPIOs were diluted in saline in 8 steps to concentrations of 0 to $0.7 \mathrm{mmol} \mathrm{Fe} / \mathrm{L}$. The $R_{1}, R_{2}$ and $R_{2}^{*}$ of each sample were determined using a series of IR (TE $=8.1 \mathrm{~ms}$; TR $=$ $10000 \mathrm{~s} ; \mathrm{TI}=150,250,500,750,1000,1500,2000,2500,3000$ and $3500 \mathrm{~ms}$ ), SE (TE $=11,22,33,44,55,66,77,88,99,110,121$ and $132 \mathrm{~ms}$; TR = 10000) and GE (TE = $3.9,7.5,10,15,20,25,30$ and $40 \mathrm{~ms} ; \mathrm{TR}=10000 ; \mathrm{FA}=40^{\circ}$ ) acquisitions, respectively. Before acquisition, first and second order global shimming was performed. All data were acquired at room temperature.

The cNGR peptide used in this study was biotinylated and could therefore be easily linked to the SPIOs. The iron oxide nanoparticles are able to bind $100 \mathrm{nmol} /$ $\mathrm{ml}$ biotinylated compounds, as indicated by the manufacturer. For the in vivo experiment, a dose of $200 \mu \mathrm{L}$ was prepared. The peptide was dissolved in water and $20 \mathrm{nmol}$ peptide was added to the iron nanoparticles. This resulted in a total dose of approximately $29 \mu \mathrm{mol}$ Fe/kg body weight. Unlabeled SPIO contrast agent was used as control.

\section{Animal model}

The animal study was approved by the institutional animal welfare committee. Human colorectal adenocarcinoma cells (1.5-3.0-106; LS174T; American Type Culture Collection CL-188) were subcutaneously injected in one flank of 12-weekold male Swiss nu/nu mice (Charles River Laboratories, L'Arbresle cedex, France). Mice were subjected to MRI when the tumor volume was approximately $1 \mathrm{~cm}^{3}$, which was 14 days after LS174T injection. For in vivo MRI, mice were anesthetized using 1.5-2.0\% isoflurane (Abbott Laboratories Ltd Queensborough, UK) in medical air and were placed in prone position in a dedicated animal holder with a built-in mask for anesthesia gas supply. An infusion line was placed in the jugular vein for contrast agent administration during MRI. A heating pad was placed over the mice to maintain normothermic conditions. Respiration rate and body temperature were continuously monitored via a balloon sensor and a rectal temperature probe, respectively, interfaced to an MR compatible small animal monitoring system (SA Instruments, Inc., Stony Brook, NY, USA). Mice were randomly selected for injection with either cNGR-labeled SPIOs or unlabeled SPIOs. For both contrast agent groups, five mice were included. The investigator was blinded for the administered contrast agent. After the MRI experiment, all mice were euthanized by exsanguination and perfusion fixed with $2 \%$ glutaraldehyde. Tumors were excised, sliced in approximately $1 \mathrm{~mm}$ 
slices corresponding with the MRI geometry and conserved in $2 \%$ glutaraldehyde for histology.

\section{In vivo MRI experiment}

For animal experiments, the 7.0 Tesla MRI system was equipped with a BGA6-S microimaging gradient system (maximum gradient strength, $1000 \mathrm{mTm}^{-1}$; slew rate, $11250 \mathrm{Tm}^{-1} \mathrm{~s}^{-1}$ ) and a quadrature volume resonator (inner diameter, $35 \mathrm{~mm}$ ). Pre and post contrast images were acquired with a FOV of $30 \times 30 \mathrm{~mm}^{2}, 192 \times 256$ acquisition matrix, $256 \times 256$ reconstruction matrix, $0.12 \times 0.12 \mathrm{~mm}^{2}$ voxel size and $1 \mathrm{~mm}$ slice thickness. In the tumor, 6 to 8 axial slices were planned depending on the dimensions and orientation of the tumor. The order of the scans was randomized for each animal and consisted of a $T_{2}$-weighted spin echo sequence for tumor localization (TE=30 ms and $T R=3500 \mathrm{~ms}$ ), a GE series (TE=2.9, 6, 10, 15, $25 \mathrm{~ms}$, $T R=750 \mathrm{~ms}$ and $F A=40^{\circ}$ ), and a White Marker series (TE=5 ms, TR=750 ms, rephasing 50, 25 and 0\%). To determine the plasma half-life of the contrast medium, a dynamic GE series (TE=4.0 ms, TR=7.0 ms, $6.7 \mathrm{~s}$ per dynamic phase, $\mathrm{FOV}=30 \times 30 \mathrm{~mm}^{2}$, matrix $=64 \times 64$ ) was acquired with 100 dynamic phases, $200 \mu \mathrm{L}$ of contrast agent was administered after the 20th dynamic phase inside the magnet. Post contrast imaging was started two minutes after the dynamic scan.

\section{Histology}

The fixed tumor tissue was processed overnight in the Tissue-Tek V.I.P. apparatus (Sakura, Zoeterwoude, the Netherlands) where it was dehydrated and permeabilized for paraffin perfusion. After paraffin perfusion, the tissues were blocked in paraffin and sections $(4 \mu \mathrm{m})$ were cut on a microtome (Leica Microsystems, Nussloch, Germany). Iron-staining was performed on tissue sections according to Perls, with solution A containing 2 g Potassiumferrocyanidell (Merck, Darmstadt, Germany) in $100 \mathrm{ml}$ aqua dest, and solution B being 2\% HCl (Merck, Darmstadt, Germany). Before staining, equal volumes of solution $A$ and $B$ were filtrated through a paper filter (Schleicher\&Schuell, Dassel, Germany). Tissue sections were deparaffinized in xylene (Merck, Darmstadt, Germany ) and rehydrated in a series of alcohol $(100 \%$ $\rightarrow 0 \%$ ). Next, the slides were incubated in Perls staining solution for 30 min and counterstained with Nuclear Fast Red (Klinipath, Duiven, the Netherlands) for 5 min. After rinsing in aqua dest, the slides were dehydrated in a series of alcohol $(0 \rightarrow 100 \%)$ and xylene and mounted on a microscope glass with Entellan (Merck, Darmstadt, Germany). Slides were examined on a Leica DM3000 microscope equipped with a DFC320 camera.

\section{Data analysis}

$R_{2}^{*}$ relaxation rate maps were calculated from the series of five GE acquisitions. The complex image data of these images was subsequently used to compute the SGM images (Figure 2.1 panel B). SGM uses a shifting window (size, 5 voxels) in which Fourier transformation of the complex image data was performed ${ }^{[25]}$. The spectral shift in the obtained Fourier spectrum with respect to the center of the spectrum is proportional to the susceptibility gradient. The spectral shift was determined by 
calculating the center of mass of the spectrum and was assigned to the center voxel of the shifting window in the calculated SGM image. The SGM image contained two susceptibility gradient vectors originating from the $x$ - and $y$-direction in each voxel. The susceptibility gradient in each voxel was calculated by taking the length of the resulting vector.

Regions of interest (ROI) were drawn manually using MRIcro ${ }^{[26]}$. For the single cylinder phantom, two ROls were drawn, the first in the positive contrast regions and the second in a non-positive contrast region inside the phantom. The contrast-to-noise ratio (CNR) for SGM, White Marker and GE was determined by $C N R=\frac{\left|S_{A}-S_{B}\right|}{\sigma}$, where $S_{A}$ and $S_{B}$ are the mean values of the ROIs (the positive contrast area ănd the non-positive contrast area, respectively) and $\sigma$ is the standard deviation of the nonpositive contrast ROI. For the multiple cylinder phantom, one ROI was drawn covering the entire cross-section of the tube, 9 equally sized ROls were drawn for the iron oxide cylinders and the direct vicinity, and ROI was drawn in the rim of the tube to obtain background signal outside the positive contrast regions. Enhanced voxels (EVs) were selected for each positive contrast technique, which was defined as the $5 \%$ voxels with the strongest contrast agent induced signal of the tube. CNR was calculated for each concentration by using only EVs, which were within the ROI (iron oxide cylinder and vicinity). For the in vivo images of the different acquisition techniques, ROIs were drawn in the rim and core of each tumor. Again, only enhanced voxels (EVs) were considered during image analysis, which was defined as the $5 \%$ voxels with the strongest contrast agent induced signal differences between post- and pre-contrast images. All voxel intensity values from each animal and each group (per contrast agent type) were collected to produce a histogram. From this histogram the $5 \%$ most strongly enhanced voxel values were taken. For these voxels, the probability of being part of the non-enhanced background tissue is $5 \%$ or less. Increasing this cut off percentage to $10 \%$ did not essentially modify the results of the comparison study. For the in vivo measurements, the signal difference $S_{A}-S_{B}$ was defined as the mean pixel difference of the post- and pre-contrast images of the EVs. For $\sigma$, the standard deviation of the pre-contrast signal in the contralateral non-angiogenic muscle tissue was taken. Comparison of the different imaging techniques was restricted to those acquisitions where cNGR-labeled SPIOs were administered.

\section{Statistical analysis}

First, the number of EVs for rim and core was compared with a non-parametric Wilcoxon signed-rank test and the ratio for unlabeled and cNGR-labeled SPIOs was tested with a non-parametric Mann-Whitney $U$ test. Second, the number of EVs as a function of the distance of the tumor contour were evaluated with spatial linear regression analysis. A significant (negative) correlation would indicate a rim-core difference. The intercept resulting from the regression analysis was used to compare the rim uptake of labeled and unlabeled SPIOs. All statistical tests were performed on the results of $R_{2}{ }^{*}, S G M$ and GE using a commercial statistical software package (SPSS 16, SPSS Inc., Chicago, IL, USA). P $<0.05$ was considered statistically significant. 


\subsection{Results}

\section{Phantom MRI experiment}

For $\mathrm{GE}, R_{2}{ }^{*}$ relaxation rate mapping, White Marker and SGM, representative images of the single cylinder phantom are presented in Figure 2.2. The $R_{2}{ }^{*}$ relaxation rate was calculated in a region of interest (ROI) on an axial slice. The $R_{2}^{*}$ contrast between the iron oxide filled cylindrical core and the agarose filled outer shell of the phantom is visible with this technique (Figure 2.2B). For the White Marker images, coronal slices were acquired, since the gradient caused by the iron needs to be in the slice direction to generate positive contrast with this technique. The positive contrast is noticeable along the cylinder axis (Figure 2.2C-F).For SGM, the strongest positive contrast arises at the interface between the iron-oxide filled core and the agarose filled outer shell of the phantom. This is because the SGM technique visualizes the susceptibility gradient, which is only present in the direct vicinity outside the iron oxide core (Figure 2.2G-J).
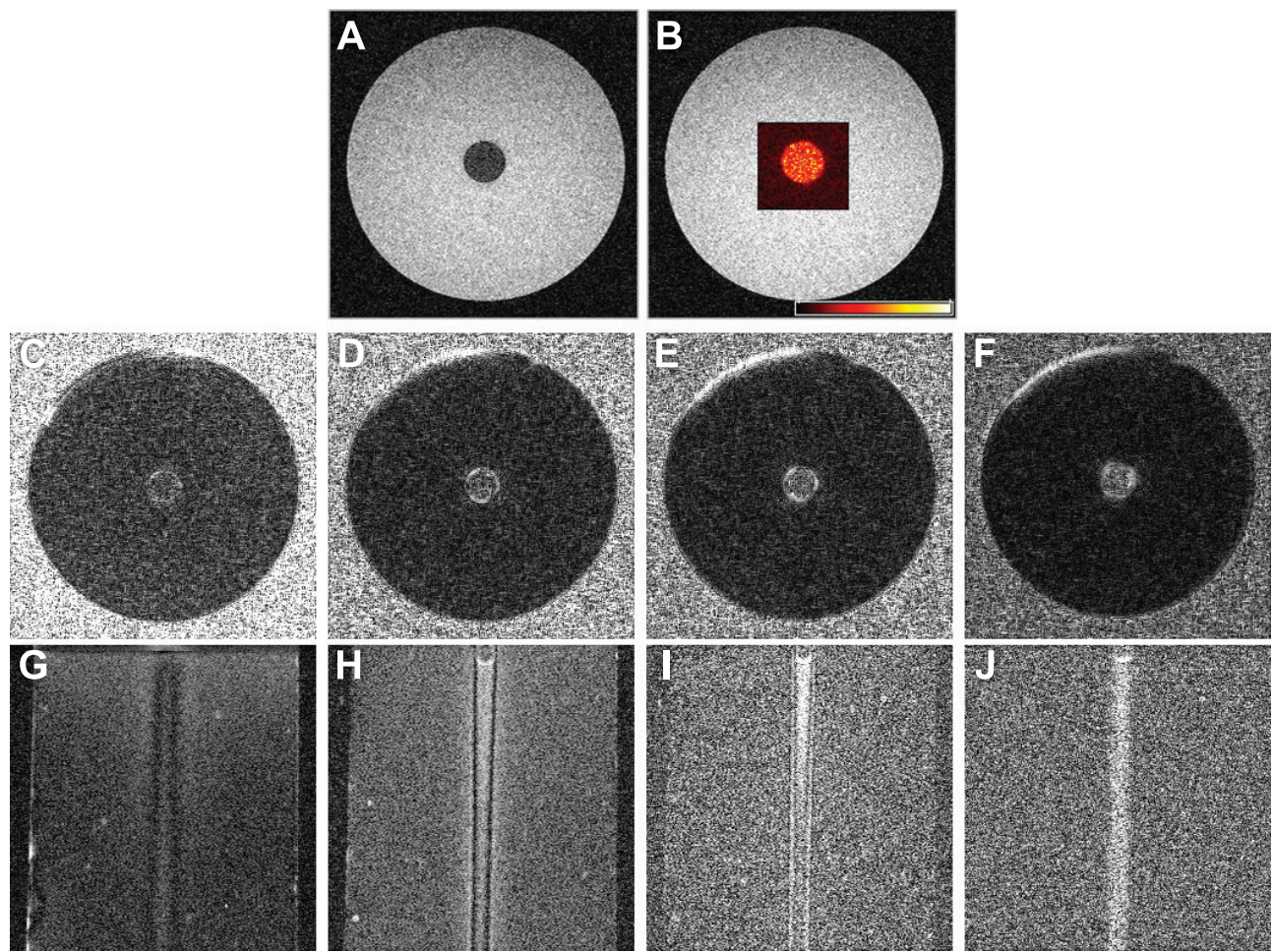

Figure 2.2 - A) Conventional axial gradient echo image (TE, $7 \mathrm{~ms}$ ) of the cylindrical agarose phantom with an iron oxide filled core. B) $R_{2}{ }^{*}$ relaxation rate map, range from 0 to $100 \mathrm{~s}^{-1}$. (C-F) Coronal sections of the phantom for the White Marker with different percentages of the rephasing gradient strength $75 \%(C), 50 \%$ (D), 25\% (E), and 0\% (F). (G-J) SGM images with different TE of $3.5 \mathrm{~ms}(\mathrm{G}), 7 \mathrm{~ms}(\mathrm{H}), 10 \mathrm{~ms}(\mathrm{I})$, and $15 \mathrm{~ms}(\mathrm{~J})$. Note that the orientation of the White Marker slices is different from those of the GE and SGM images as the cylinder was equally positioned along the $B_{0}$ field. The White Marker technique is only sensitive to susceptibility gradients in the slice selection direction. 
CNR values for the various acquisition techniques for the single cylinder phantom are presented in Table 2.1. For the positive contrast techniques, the highest CNR of 5.3 was obtained with the SGM sequence with a TE of $15 \mathrm{~ms}$. For White Marker, the highest CNR of 2.3 was obtained with $25 \%$ rephasing.

For the multiple cylinder phantom experiment, the CNR of the SGM images showed a linear relation (highest slope $9.10 \pm 0.38 \mathrm{~L} / \mathrm{mmol} \mathrm{Fe}$ at TE $=7 \mathrm{~ms}, R^{2}=0.99$ ) with the iron oxide concentration at various echo times (Figure 2.3E). For White Marker the most sensitive linear relation (highest slope $0.41 \pm 0.17 \mathrm{~L} / \mathrm{mmol} \mathrm{Fe}, R^{2}=0.47$ ) was found for $50 \%$ rephasing, with a small positive slope (Figure $2.3 \mathrm{~F}$ ).

Table 2.1 CNR values of the cylindrical agarose phantom with iron oxide core, measured for SGM and White Marker with different settings.

\begin{tabular}{cccc}
\hline \multicolumn{2}{c}{ White Marker } & \multicolumn{3}{c}{ SGM } \\
\hline Rephasing (\%) & CNR $(-)$ & TE $(\mathrm{ms})$ & CNR $(-)$ \\
0 & 1.6 & 3.5 & 1.6 \\
25 & 2.3 & 7 & 4.2 \\
50 & 2.2 & 10 & 4.9 \\
75 & 1.8 & 15 & 5.3 \\
& & 25 & 4.7 \\
\hline
\end{tabular}

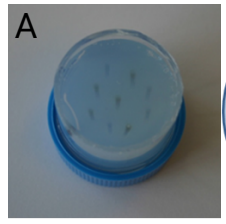

E

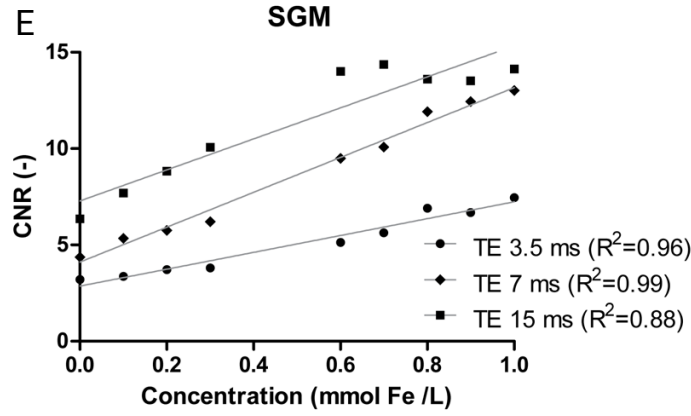

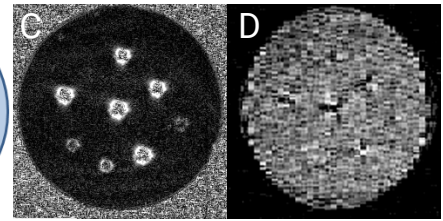

White Marker

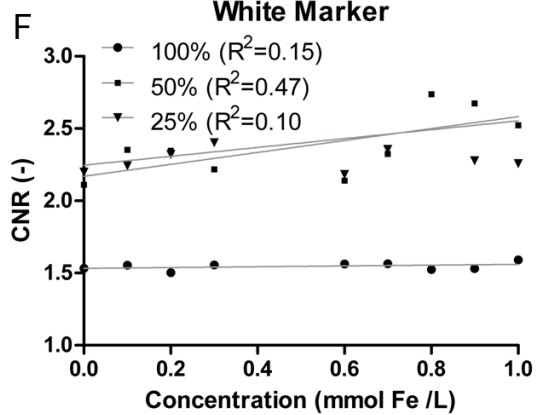

Figure 2.3 - A) Photograph of the (multiple cylinder) phantom with the iron oxide filled cylinders. B) Scheme of the iron oxide concentration at each position (in mmol Fe /L). C) Axial (short-axis) SGM image (TE $15 \mathrm{~ms}$, TR $750 \mathrm{~ms}$ ). Positive contrast is shown in the direct vicinity of the cylinder; at higher iron oxide concentration the positive contrast becomes stronger. D) Axial (short-axis) White Marker image (50\% rephasing), obtained by interpolation and reformation of two orthogonally acquired long-axis images. E \& F) CNR of SGM and White Marker as function of the concentration for different rephasing values or echo times. 


\section{Animal experiments}

Contrast agent characterization. The relaxivities $r_{1}, r_{2}$ and $r_{2}{ }^{*}$ at $7.0 T$ were $1.30 \pm 0.13$ $\left(R^{2}=0.951\right), 196 \pm 9.4\left(R^{2}=0.987\right)$ and $416 \pm 11\left(R^{2}=0.996\right) \mathrm{s}^{-1} \mathrm{mM}-1$ Fe, respectively. The hydrodynamic diameter of the SPIO particles was $86.3 \mathrm{~nm}$ with a polydispersity index of 0.13 , as measured using dynamic light scattering. For cNGR-labeled SPIOs, the plasma half-life was $63.3 \pm 13.6 \mathrm{~s}$ and for the unlabeled SPIO $64.8 \pm 12.1 \mathrm{~s}$, as determined from an exponential fit of the signal decay curve in the aorta.

Positive contrast imaging in tumors. Figure 2.4 shows representative images of a tumor-bearing mouse injected with cNGR-SPIO contrast agent for the different imaging techniques. For the GE and SGM, but not for White Marker images, the effect of contrast uptake is noticeable when pre- and post-contrast images are compared. The post contrast GE and SGM images show hypointense regions at exactly the same position. An $R_{2}^{*}$ post- and pre-contrast subtraction image, $\Delta R_{2}{ }^{*}$, was calculated and showed increased relaxations rates in panel B3 (Figure 2.4). Also the SGM subtraction image, $\triangle S G M$, showed an increased susceptibility gradient in panel D3 (Figure 2.4). White Marker displayed a weak contrast, which could not be correlated with the hyperintensities of the other techniques.

Histology. Perls iron staining was performed on a number of slices of the excised tumors, where the blue coloring indicates iron oxide accumulation. Examination of the slices showed a spatial correspondence with the MRI acquisitions. Blue coloring occurs mainly in the rim and could be related to the contrast enhancement on the in vivo post contrast MRI (Figure 2.5).

Contrast-to-noise ratio. For SGM and GE images, the highest CNR values in tumor bearing mice were $6.2 \pm 0.5$ and $5.7 \pm 0.9$, respectively, whereas the best White Marker images had a CNR of 1.2 \pm 0.1 (Table 2.2). The CNR of SGM was slightly higher than for conventional GE images, although it was not significantly different. The CNR of White Marker was significantly lower than for SGM and GE $(P<0.05)$.

Rim and core analysis. Figure 2.6 shows a diagram of the percentage of EVs, as determined by the $\Delta R_{2}{ }^{*}$ measurements, as a function of the distance to the tumor periphery. The mean percentage EVs decreases from 0 to $1 \mathrm{~mm}$ distance for both labeled SPIOs and unlabeled SPIOs according to linear regression analysis $\left(R^{2}=0.97\right.$ for labeled SPIOs and $R^{2}=0.86$ for unlabeled SPIOs). Furthermore, linear regression analysis showed a higher intercept in the percentage of EVs for the cNGR-labeled SPIOs compared to the unlabeled SPIOs $(p<0.001)$, which indicates a significantly higher accumulation of cNGR-labeled SPIOs in the rim compared to the unlabeled SPIOs. The same analysis was performed for SGM (TE=6 ms), which also showed the decrease $\left(R^{2}=0.95\right.$ for both contrast agents) in EVs as a function of distance and a significantly higher intercept for the cNGR-labeled SPIOs $(p<0.001)$. The percentage of EVs remained approximately constant beyond $1 \mathrm{~mm}$. Therefore, the thickness of the rim was chosen as $1 \mathrm{~mm}$ to further evaluate the differences between CNGR and unlabeled SPIOs over the tumor rim and core [22,27,28]. Ligand efficacy was further analyzed by comparing the number of EVs in the $1 \mathrm{~mm}$ 

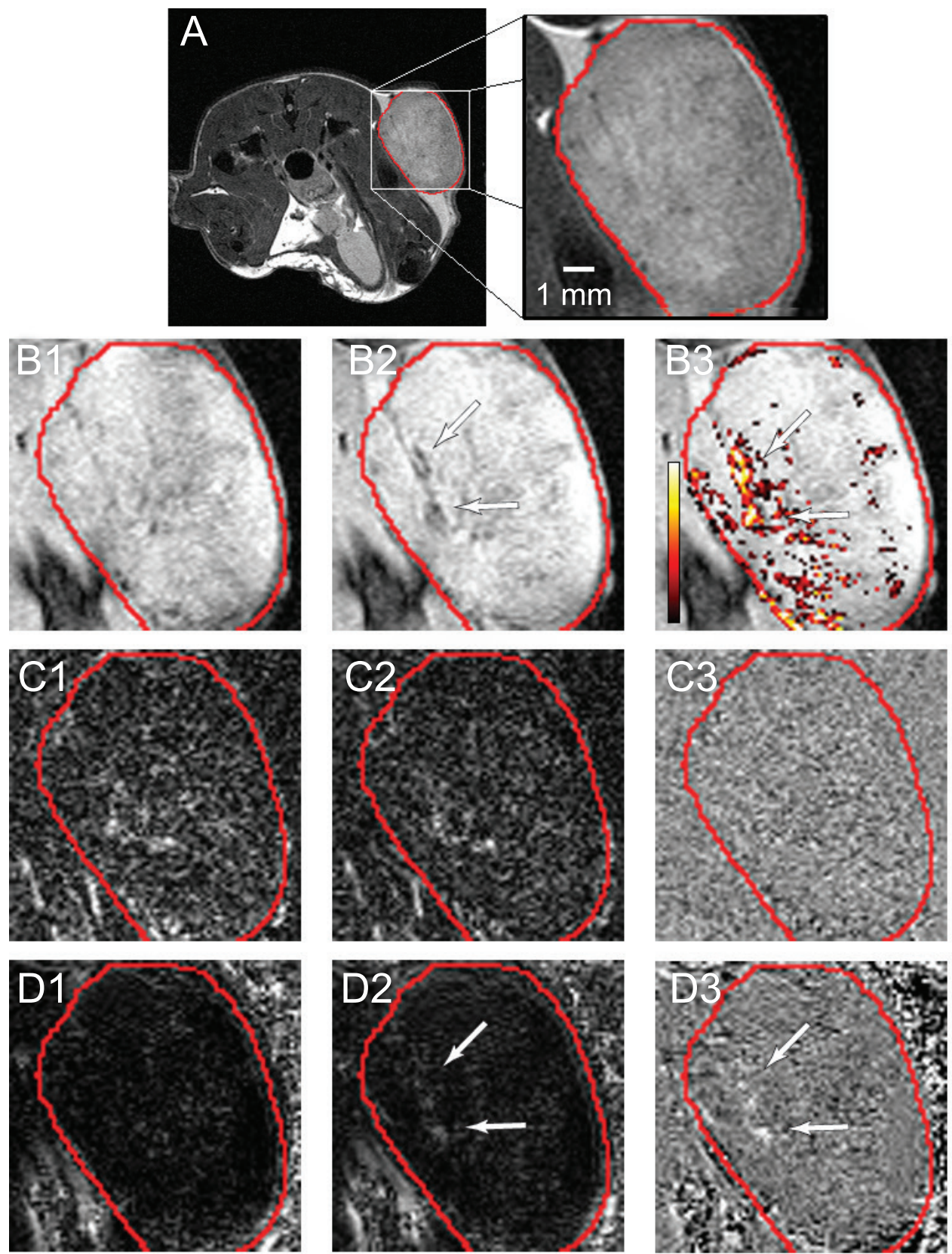

Figure 2.4 - Axial sections through the tumor, where the red line represents the tumor contour and the purple line divides the rim and core section (1 mm thick rim). A) A spin echo image that shows the location of the tumor in a mouse of the CNGR-SPIO group. B1 and B2) Pre and post contrast GE images, respectively, B3) $\Delta R_{2}{ }^{*}$ mapping (post-pre contrast), color bar range is 0 (black) to 100 (white) $\mathrm{s}^{-1}$. C1 and C2) Pre and post contrast White Marker images with $0 \%$ rephasing, respectively, C3) $\Delta$ White Marker (post-pre contrast). D1 and D2) Pre and post contrast SGM TE $=6 \mathrm{~ms}$, respectively, D3) $\triangle \mathrm{SGM}$ image (post-pre contrast). The arrows show SPIO accumulation in different images. 
thick rim and core for $R_{2}{ }^{*}$ mapping, SGM (TE $\left.=6 \mathrm{~ms}\right)$ and the GE image (TE = $6 \mathrm{~ms}$ ) (Figure 2.7). Significantly stronger rim enhancement relative to the core was found for all three techniques and both contrast agents $(P<0.05)$, except for unlabeled SPIOs in the GE images. The stronger enhancement in the rim compared to the core reflects the higher level of angiogenic activity and blood volume typically found in the tumor rim ${ }^{[22,29]}$.

Table 2.2 - In vivo CNR values for tumor bearing mice injected with cNGR-labeled SPIOs for GE, White Marker en SGM acquisitions with different echo time or rephasing.

\begin{tabular}{cccccc}
\hline \multicolumn{2}{c}{ GE } & \multicolumn{2}{c}{ White Marker } & \multicolumn{2}{c}{ SGM } \\
TE $(\mathrm{ms})$ & CNR (-) & Rephasing(\%) & CNR (-) & TE (ms) & CNR (-) \\
\hline 3 & $5.1 \pm 0.5$ & 0 & $1.2 \pm 0.1$ & 3 & $4.9 \pm 0.4$ \\
6 & $5.7 \pm 0.9$ & 25 & $1.2 \pm 0.1$ & 6 & $6.2 \pm 0.5$ \\
10 & $3.9 \pm 0.6$ & 50 & $1.2 \pm 0.1$ & 10 & $5.7 \pm 0.7$ \\
15 & $3.1 \pm 0.5$ & & & 15 & $4.8 \pm 0.7$ \\
\hline
\end{tabular}

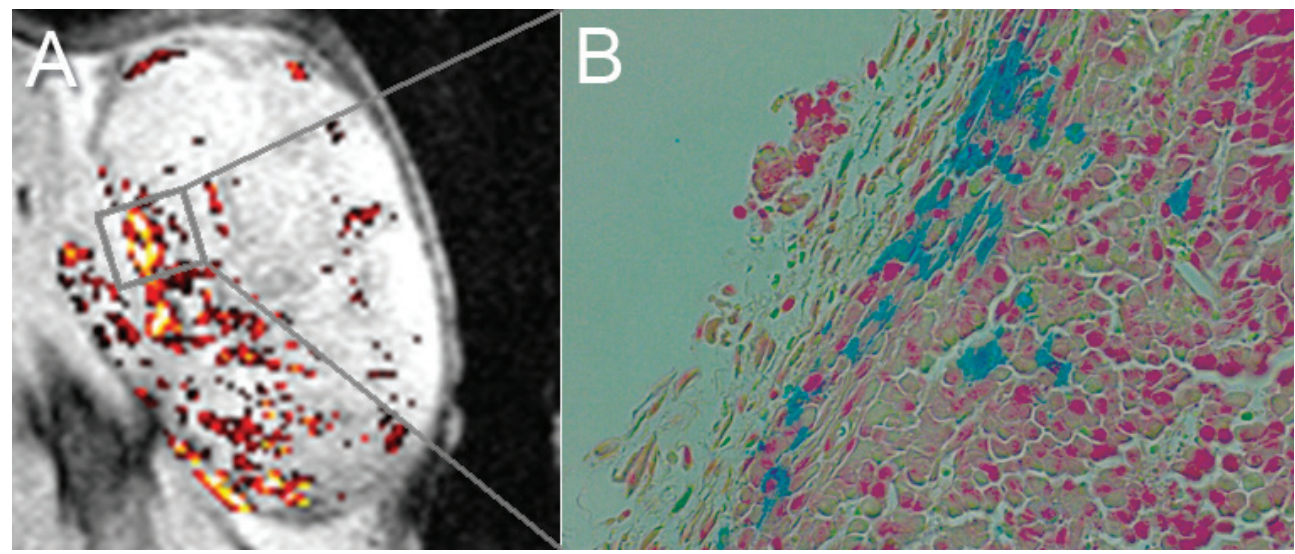

Figure 2.5 - Comparison of contrast-enhanced MRI with histology. The box in the $\Delta R_{2}{ }^{*}$ mapping (A) shows the location of the corresponding histological tissue section (B). The tumor tissue slice in panel $B$ is stained for iron (Perls blue) and shows iron oxide accumulation in the tumor rim. 


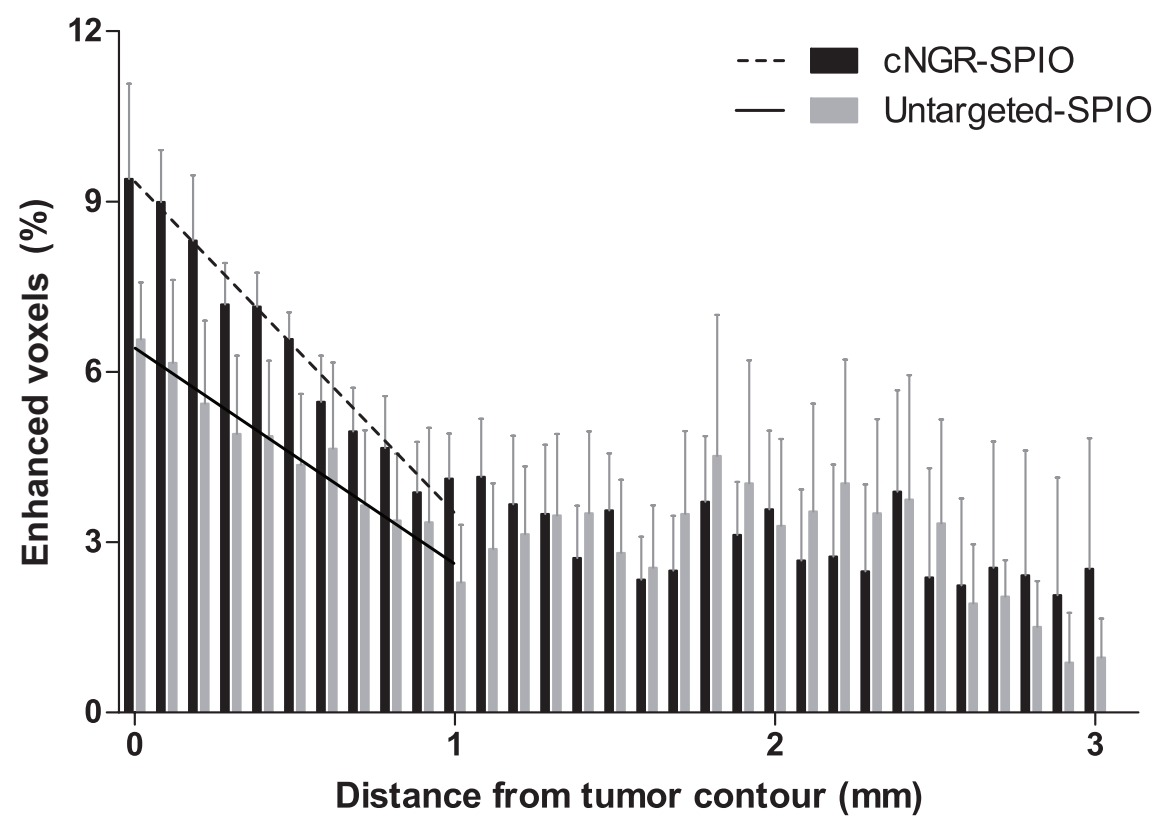

Figure 2.6 The percentage of EVs versus the distance to the tumor contour with relaxation rate mapping $\left(\Delta R_{2}^{*}\right)$. There is a deceasing trend from 0 to $1 \mathrm{~mm}$. Based on this observation, $1 \mathrm{~mm}$ was chosen as the thickness of the rim. Data are presented as mean \pm standard error.

- cNGR-SPIO Untargeted-SPIO

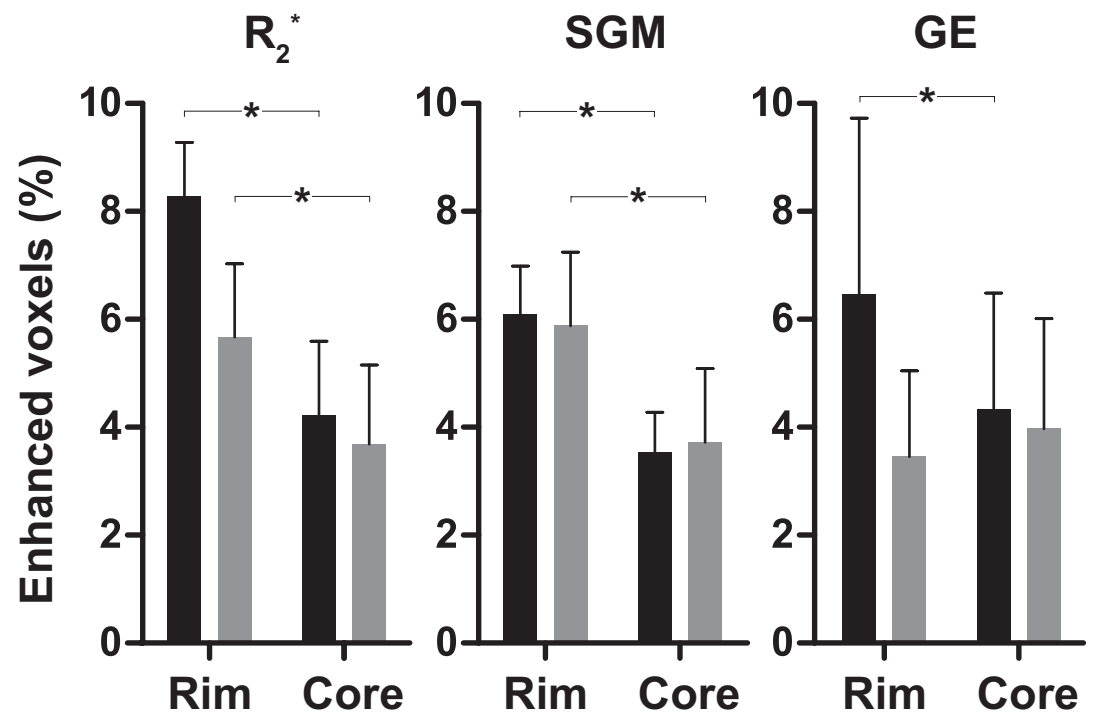

Figure 2.7 - The percentage of EV at optimal settings (SGM and GE: TE $6.0 \mathrm{~ms}$ ) for all techniques. The percentage of EVs is given for rim and core and for both contrast agent groups. Significant differences were found between rim and core for all three techniques and both contrast agents (* $\mathrm{P}<0.05)$, except for untargeted-SPIO in the GE image. Data are presented as mean \pm standard error. 


\subsection{Discussion}

\section{Current findings}

In this study, two positive contrast MRI techniques to detect iron oxide contrast agents, White Marker and SGM, were compared with GE imaging and $R_{2}^{*}$ mapping in cylindrical phantoms and in tumor-bearing mice. In both experiments, SPIOs could be detected with positive contrast techniques. First, the White Marker and the SGM technique were optimized for a phantom to obtain the strongest positive contrast between ironoxide filled regions and the agarose filled background. The in-vitro MRI sensitivity, in terms of signal increase per iron oxide concentration unit, was much higher for SGM than White Marker (range 0 to $1 \mathrm{mmol} F e / L$ ). Second, in-vivo experiments with these optimized techniques showed that only SGM, and not White Marker, provided positive contrast, which was comparable to $R_{2}{ }^{*}$ mapping in terms of signal effect and localization. Third, a stronger uptake of cNGR-labeled SPIOs in the 1-mm thick tumor rim relative to the core region was observed for SGM and $R_{2}^{*}$ mapping. Fourth, both $\mathrm{MRI}$ and histology demonstrated accumulation of cNGR-labeled SPIOs mainly in the tumor. Fifth, spatial linear regression analysis showed a significantly higher uptake of cNGR-labeled SPIOs relative to unlabeled SPIOs in the outermost part of the rim for $R_{2}^{*}$ mapping and SGM.

\section{In vivo versus in vitro imaging}

Different results were found in the in-vivo and in-vitro experiments for the positive contrast techniques. In the phantom experiments, SGM achieved the strongest positive contrast at an echo time of $15 \mathrm{~ms}$, whereas in-vivo the optimal echo time was found to be $6 \mathrm{~ms}$. The differences between these results are probably related to in-vivo motion artifacts and geometric differences. With an increasing echo time the motion artifacts will also increase. Therefore, the optimal positive contrast for in-vivo SGM will shift to lower echo times. Furthermore, some artifacts appear outside the $\mathrm{ROI}$ in both the pre and post contrast SGM images due to tissue air interfaces for the intestines and the skin.

White Marker showed positive contrast in the phantom, whereas hardly any contrast was found in-vivo. The differences between in-vivo CNR and in-vitro CNR for White Marker are possibly due to differences in the induced susceptibility gradient of the iron oxide. The strength of the magnetic perturbation depends on a number of parameters, including magnetic properties of the iron particles and shape and dimension of the iron particle distribution. The magnetic field perturbation of a spherical object filled with iron, which most closely reflects the in-vivo configuration of the contrast agent accumulation, is approximately 10 -fold smaller compared to an infinite cylindrical phantom having the same radius and filled with the same substance. Furthermore, the diameter of the in-vivo sphere is much smaller than the cylindrical phantom, resulting in a smaller magnetic field perturbation. The phantom in this study does not mimic the in-vivo angiogenesis situation. However, this phantom induces geometrically well-defined susceptibility gradients and therefore it was able to show how positive contrast was induced by White Marker and SGM. 


\section{Comparison of different MRI techniques}

The analysis was performed on the EVs, which were selected separately for each technique. This selection criterion was chosen to avoid spatial mismatching as positive contrast manifests themselves in different regions and levels for the different techniques. Even with the relatively low iron concentration of $29 \mu \mathrm{mol} \mathrm{Fe} / \mathrm{kg}$ body weight, the in-vivo CNR for SGM was still greater than 5, which is assumed as the lower detection limit for molecular imaging [30]. The high relaxivities of SPIOs have the opportunity to induce stronger contrast at higher concentrations, which was shown by Zhang et al. ${ }^{[6]}$ who used a more than 30 times higher concentration than in the present study. White Marker did not show positive contrast in tumor areas where contrast was observed with $R_{2}^{*}$ mapping, GE or SGM (Figure 2.4). The absence of invivo positive contrast for White Marker might be explained as follows. White Marker gives positive contrast when a gradient field in the slice direction compensates the refocusing gradient. Magnetic fields with non-matching strengths or directions will not contribute to the refocusing of the spins. Matching of this gradient field depends on three parameters: the strength of the refocusing gradient, the echo time and the strength of the additional field induced by the iron oxide. This field is derived from the susceptibility gradient, which means that only a part of the gradient will contribute to an effective rephasing. Likely, this contribution is too small to be detected in-vivo. Recently, Varma et al. ${ }^{[31]}$ introduced a White Marker post-processing approach with a $3 \mathrm{D}$ acquisition, which might resolve these issues.

Small voxel sizes are preferred to measure the spatial pattern of susceptibility effects, because it minimizes contributions of intravoxel dephasing as much as possible. To obtain small voxels a 3D acquisition with isotropic voxels can be used, with the advantage of calculating three susceptibility gradients with SGM and a higher signalto-noise ratio (SNR), compared to a 2D acquisition. However, the acquisition time of a $3 \mathrm{D}$ acquisition is longer and an extra phase encoding is involved, which is prone to motion artifacts. In this study, a 2D GE acquisition was therefore chosen to achieve a reasonable acquisition time for the in-vivo experiments. With this 2D acquisition, SGM provided promising results and a reasonable CNR in-vivo. The characteristic properties of all three techniques evaluated in this study are summarized in Table 2.3.

Table 2.3 Characteristics of the applied susceptibility imaging techniques

\begin{tabular}{lccc}
\hline & $\boldsymbol{R}_{2}{ }^{*}$ mapping & White Marker & SGM \\
\hline $\begin{array}{l}\text { Physical principle of } \\
\text { contrast }\end{array}$ & $T_{2}{ }^{*}$ Shortening & $\Delta \mathrm{B}_{\mathrm{z}}$ & $\Delta \mathrm{G}_{\mathrm{x}, \mathrm{y}, \mathrm{z}}$ \\
Directional sensitivity & $\mathrm{No}$ & Yes & Yes \\
2D and/or 3D & $2 \mathrm{D}$ and 3D & $2 \mathrm{D}$ only ${ }^{(1)}$ & $2 \mathrm{D}$ and 3D \\
Anatomical information & Yes & No or reduced & Yes \\
Acquisition time $(\mathbf{s})$ & $5 \times 144^{(2)}$ & 144 & 144 \\
\hline
\end{tabular}

${ }^{1}$ A 3D acquisition can be used for White Marker post-processing.

25 different echo times were used for $R_{2}^{*}$ mapping. 


\section{Limitations}

The plasma half-life of both SPIO contrast agents was approximately 63 seconds. The amount of homing of a labeled SPIO contrast agent to the vascular target is likely lower compared with other targeted contrast agent with longer plasma half-life due to the rapid uptake into the reticuloendothelial system, but further histology and biodistribution measurements are needed to substantiate this hypothesis. Despite the short plasma half-life, linear regression analysis in the rim region showed significant differences between labeled and unlabeled SPIOs (Figure 2.6). In addition, as a result of the short plasma half-life, post contrast imaging could be started 10 minutes after contrast injection and a good target-to-background ratio was achieved. Furthermore, histological validation was performed on a few slices only, since the homing of cNGR conjugated to different particles was already previously validated by histology by different research groups ${ }^{[18,22,32]}$.

\section{Clinical perspective}

Positive contrast techniques using SPIO contrast agents, especially SGM, have a number of potential clinical advantages for future molecular MRI. SGM produces positive contrast images, which are preferred in the reading of radiological images. These SGM images are comparable to GE images in terms of CNR and acquisition time. This is important because eventual clinical implementation of molecular MRI requires rapid imaging techniques. Furthermore, iron oxide particles-, have already been applied in humans in clinical trials ${ }^{[33]}$.

\section{Conclusion}

This experimental study shows the in-vivo efficacy of positive contrast imaging techniques for molecular MRI using a cNGR-SPIO contrast agent targeted to the angiogenic vasculature of murine tumors. Of the positive contrast imaging techniques evaluated, SGM is most promising for molecular imaging. Further research is required to optimize the imaging technique and the design of the iron oxide particles before translation to clinical trials can be made. 


\section{References}

1. Massoud TF, Gambhir SS. Molecular imaging in living subjects: seeing fundamental biological processes in a new light. Genes Dev 2003;17(5):545-580.

2. Gore JC, Yankeelov TE, Peterson TE, Avison MJ. Molecular imaging without radiopharmaceuticals? J Nucl Med 2009;50(6):999-1007.

3. Mulder WJ, Strijkers GJ, van Tilborg GA, Cormode DP, Fayad ZA, Nicolay K. Nanoparticulate assemblies of amphiphiles and diagnostically active materials for multimodality imaging. Acc Chem Res 2009;42(7):904-914.

4. Aime S, Cabella C, Colombatto S, Geninatti Crich S, Gianolio E, Maggioni F. Insights into the use of paramagnetic Gd(III) complexes in MR-molecular imaging investigations. J Magn Reson Imaging 2002;16(4):394-406.

5. Artemov D. Molecular magnetic resonance imaging with targeted contrast agents. J Cell Biochem 2003;90(3):518-524.

6. Zhang C, Jugold M, Woenne EC, Lammers T, Morgenstern B, Mueller MM, Zentgraf $H$, Bock M, Eisenhut M, Semmler W, Kiessling F. Specific targeting of tumor angiogenesis by RGD-conjugated ultrasmall superparamagnetic iron oxide particles using a clinical 1.5-T magnetic resonance scanner. Cancer Res 2007;67(4):1555-1562.

7. Seppenwoolde JH, Viergever MA, Bakker CJ. Passive tracking exploiting local signal conservation: the white marker phenomenon. Magn Reson Med 2003;50(4):784-790.

8. Mani V, Briley-Saebo KC, Itskovich VV, Samber DD, Fayad ZA. Gradient echo acquisition for superparamagnetic particles with positive contrast (GRASP): sequence characterization in membrane and glass superparamagnetic iron oxide phantoms at 1.5T and 3T. Magn Reson Med 2006;55(1):126-135.

9. Cunningham $\mathrm{CH}$, Arai T, Yang PC, McConnell MV, Pauly JM, Conolly SM. Positive contrast magnetic resonance imaging of cells labeled with magnetic nanoparticles. Magn Reson Med 2005;53(5):999-1005.

10. Dharmakumar R, Koktzoglou I, Li D. Generating positive contrast from off-resonant spins with steady-state free precession magnetic resonance imaging: theory and proof-ofprinciple experiments. Phys Med Biol 2006;51(17):4201-4215.

11. Stuber M, Gilson WD, Schar M, Kedziorek DA, Hofmann LV, Shah S, Vonken EJ, Bulte JW, Kraitchman DL. Positive contrast visualization of iron oxide-labeled stem cells using inversion-recovery with ON-resonant water suppression (IRON). Magn Reson Med 2007;58(5):1072-1077.

12. Dahnke H, Liu W, Herzka D, Frank JA, Schaeffter T. Susceptibility gradient mapping (SGM): a new postprocessing method for positive contrast generation applied to superparamagnetic iron oxide particle (SPIO)-labeled cells. Magn Reson Med 2008;60(3):595-603.

13. Mills PH, Ahrens ET. Enhanced positive-contrast visualization of paramagnetic contrast agents using phase images. Magn Reson Med 2009;62(5):1349-1355.

14. Liu W, Dahnke H, Jordan EK, Schaeffter T, Frank JA. In-vivo MRI using positive-contrast techniques in detection of cells labeled with superparamagnetic iron oxide nanoparticles. NMR Biomed 2008;21(3):242-250. 
15. Gupta T, Virmani S, Neidt TM, Szolc-Kowalska B, Sato KT, Ryu RK, Lewandowski RJ, Gates VL, Woloschak GE, Salem R, Omary RA, Larson AC. MR tracking of iron-labeled glass radioembolization microspheres during transcatheter delivery to rabbit VX2 liver tumors: feasibility study. Radiology 2008;249(3):845-854.

16. Arap W, Pasqualini R, Ruoslahti E. Cancer treatment by targeted drug delivery to tumor vasculature in a mouse model. Science 1998;279(5349):377-380.

17. Pasqualini R, Koivunen E, Kain R, Lahdenranta J, Sakamoto M, Stryhn A, Ashmun RA, Shapiro LH, Arap W, Ruoslahti E. Aminopeptidase N is a receptor for tumor-homing peptides and a target for inhibiting angiogenesis. Cancer Res 2000;60(3):722-727.

18. Curnis F, Arrigoni G, Sacchi A, Fischetti L, Arap W, Pasqualini R, Corti A. Differential binding of drugs containing the NGR motif to CD13 isoforms in tumor vessels, epithelia, and myeloid cells. Cancer Res 2002;62(3):867-874.

19. Di Matteo P, Curnis F, Longhi R, Colombo G, Sacchi A, Crippa L, Protti MP, Ponzoni M, Toma S, Corti A. Immunogenic and structural properties of the Asn-Gly-Arg (NGR) tumor neovasculature-homing motif. Mol Immunol 2006;43(10):1509-1518.

20. Colombo G, Curnis F, De Mori GM, Gasparri A, Longoni C, Sacchi A, Longhi R, Corti A. Structure-activity relationships of linear and cyclic peptides containing the NGR tumorhoming motif. J Biol Chem 2002;277(49):47891-47897.

21. Buehler A, van Zandvoort MA, Stelt BJ, Hackeng TM, Schrans-Stassen BH, Bennaghmouch A, Hofstra L, Cleutjens JP, Duijvestijn A, Smeets MB, de Kleijn DP, Post MJ, de Muinck ED. CNGR: a novel homing sequence for CD13/APN targeted molecular imaging of murine cardiac angiogenesis in-vivo. Arterioscler Thromb Vasc Biol 2006;26(12):2681-2687.

22. Oostendorp M, Douma K, Hackeng TM, Dirksen A, Post MJ, van Zandvoort MA, Backes WH. Quantitative molecular magnetic resonance imaging of tumor angiogenesis using cNGR-labeled paramagnetic quantum dots. Cancer Res 2008;68(18):7676-7683.

23. Oostendorp M, Douma K, Wagenaar A, Slenter JM, Hackeng TM, van Zandvoort MA, Post MJ, Backes WH. Molecular magnetic resonance imaging of myocardial angiogenesis after acute myocardial infarction. Circulation 2010;121(6):775-783.

24. Artemov D, Mori N, Okollie B, Bhujwalla ZM. MR molecular imaging of the Her-2/neu receptor in breast cancer cells using targeted iron oxide nanoparticles. Magn Reson Med 2003;49(3):403-408.

25. Vonken EJ, Schar M, Stuber M. Positive contrast visualization of nitinol devices using susceptibility gradient mapping. Magn Reson Med 2008;60(3):588-594.

26. Rorden C, Brett M. Stereotaxic display of brain lesions. Behav Neurol 2000;12(4):191-200.

27. de Lussanet QG, Beets-Tan RG, Backes WH, van der Schaft DW, van Engelshoven JM, Mayo KH, Griffioen AW. Dynamic contrast-enhanced magnetic resonance imaging at 1.5 Tesla with gadopentetate dimeglumine to assess the angiostatic effects of anginex in mice. Eur J Cancer 2004;40(8):1262-1268.

28. Padhani AR. Functional MRI for anticancer therapy assessment. Eur $J$ Cancer 2002;38(16):2116-2127.

29. de Lussanet QG, Backes WH, Griffioen AW, van Engelshoven JM, Beets-Tan RG. Gadopentetate dimeglumine versus ultrasmall superparamagnetic iron oxide for dynamic contrast-enhanced MR imaging of tumor angiogenesis in human colon carcinoma in mice. Radiology 2003;229(2):429-438. 
30. Oostendorp M, Post MJ, Backes WH. Vessel growth and function: depiction with contrastenhanced MR imaging. Radiology 2009;251(2):317-335.

31. Varma G, Pedersen SF, Taupitz M, Botnar RM, Dahnke H, Keevil SF, Schaeffter T. Utilizing different methods for visualizing susceptibility from a single multi-gradient echo dataset. MAGMA 2009;22(5):297-308.

32. Pastorino F, Brignole C, Di Paolo D, Nico B, Pezzolo A, Marimpietri D, Pagnan G, Piccardi F, Cilli M, Longhi R, Ribatti D, Corti A, Allen TM, Ponzoni M. Targeting liposomal chemotherapy via both tumor cell-specific and tumor vasculature-specific ligands potentiates therapeutic efficacy. Cancer Res 2006;66(20):10073-10082.

33. Lahaye MJ, Engelen SM, Kessels AG, de Bruine AP, von Meyenfeldt MF, van Engelshoven JM, van de Velde CJ, Beets GL, Beets-Tan RG. USPIO-enhanced MR imaging for nodal staging in patients with primary rectal cancer: predictive criteria. Radiology 2008;246(3):804-811. 


\section{MRI artifacts in the ferric}

\section{model: an alternative solution}

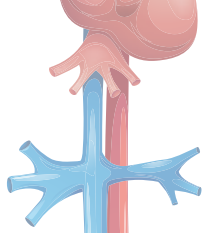

M. Wolters, R.H.M. van Hoof, A. Wagenaar, K. Douma, M.A.M.J. van Zandvoort,

T.H. Hackeng, M.J. Post, W.H. Backes, M.E. Kooi

J Thromb Haemost. 2013 Sep;11(9):1766-9.

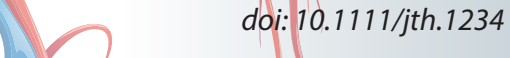

(1234
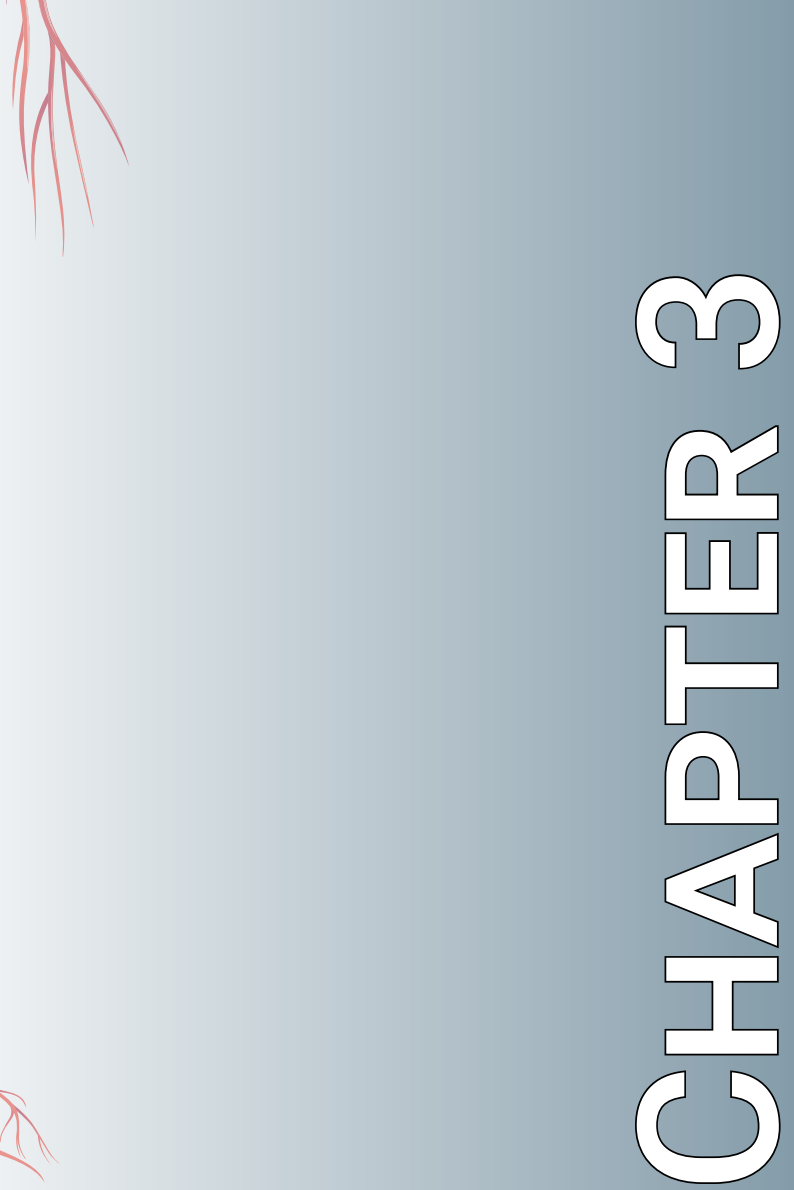
Chapter 3 


\subsection{Introduction}

Cardiovascular disease, and its related complications including thrombus formation and embolization, is a major cause of morbidity and mortality. With the current imaging techniques, i.e. ultrasound and computed tomography $\mathrm{CT}$, the origin of the thromboembolism remains undetected in $30-40 \%$ of clinical cases ${ }^{[1]}$. These techniques identify thrombi on the basis of morphological visualization and have limited ability to distinguish old from fresh thrombi. Molecular MRI might provide a solution by characterizing the molecular expression of thrombi with thrombus targeted contrast agents. Furthermore, molecular MRI has the potential to discriminate active forming clots from older thrombi ${ }^{[2,3]}$. Therefore visualization of thrombi in myocardial infarction, stroke, deep venous thrombosis, and pulmonary thromboembolism with molecular Magnetic Resonance Imaging (MRI) may improve the diagnosis and treatment.

A number of recent pre-clinical studies have demonstrated molecular MRI applications that visualized thrombi by targeting fibrin, overexpressed enzymes or activated platelets specifically in fresh thrombi of a mouse model ${ }^{[2,4-7]}$. In these studies, a widely accepted animal model for thrombus formation was used ${ }^{[8]}$, i.e. inducing a thrombus by exposing the arterial adventitia to ferric chloride $\left(\mathrm{FeCl}_{3}\right)$ for several minutes. After induction, the excess of $\mathrm{FeCl}_{3}$ is removed by rinsing the adventitia with saline. In the present study, we show that for MRI assessment the observations in this model should be interpreted with care since any residual $\mathrm{FeCl}_{3}$ can cause image artifacts (Figure $3.1 \mathrm{E}$, asterisk). $\mathrm{FeCl}_{3}$ is ferromagnetic and will locally disturb the magnetic field. These artifacts cannot be distinguished from intrinsic signal effects from the contrast agent. The non-ferromagnetic Lewis acid, $\mathrm{AlCl}_{3}$, may represent an alternative to induce thrombi without the risk of unwanted artifacts. In this study, we assess the imaging artifacts in MRI measurements of $\mathrm{FeCl}_{3}$ and $\mathrm{AlCl}_{3}$ solutions and in the thrombus animal model, where the thrombus is induced by $\mathrm{AlCl}_{3}$ or $\mathrm{FeCl}_{3}$. To compare physiological characteristics of both models, the occlusion time of the carotid artery was assessed by induction with $\mathrm{AlCl}_{3}$ or $\mathrm{FeCl}_{3}$. Furthermore, the effect of heparin during thrombus formation with $\mathrm{AlCl}_{3}$ was assessed, since a prolonged time to occlusion by heparin is a well-known feature of the $\mathrm{FeCl}_{3}$ thrombus model ${ }^{[0]}$. 


\subsection{Methods}

For the phantom experiments, vials were filled with a $\mathrm{FeCl}_{3}$ or $\mathrm{AlCl}_{3}$ solution ranging from $0.02 \%$ to $1.0 \%(\mathrm{v} / \mathrm{w})$. The vials were embedded in water and orientated perpendicularly to the static magnetic field. MR images with a $T_{1}$-weighting (Figure 3.1 A, echo time (TE)/ repetition time (TR) 3.2/100 ms, flip angle (FA) $25^{\circ}$ ) and a $T_{2}{ }^{-}$ weighting (Figure $3.1 \mathrm{~B}, \mathrm{TE} / \mathrm{TR}$ 20/1000 $\mathrm{ms}$, FA 68 ) were acquired with a gradient echo sequence, which is most sensitive to local field disturbances. Afterwards the signal-to-noise ratio (SNR) of each vial was calculated to illustrate the effect of $\mathrm{FeCl}_{3}$ and $\mathrm{AlCl}_{3}$ on the MR signal. Imaging was performed on a dedicated small-animal 7 Tesla MRI system (Bruker Biospec 70/30).

Animal experiments were approved by the institutional ethics committee on animal welfare. To assess possible differences in occlusion times, thrombi were induced in 12 weeks old Swiss mice. The right carotid artery was exposed by separating the sternocleidomastoid muscle from the trachea. Thrombus formation was induced by applying a strip of filter paper soaked in $10 \% \mathrm{FeCl}_{3}(\mathrm{n}=8)$ or $10 \% \mathrm{AlCl}_{3}(\mathrm{n}=11)$ directly on the carotid artery. After 10 minutes, the filter paper was removed and the carotid artery was rinsed with saline to remove residual $\mathrm{FeCl}_{3}$ or $\mathrm{AlCl}_{3}$. Additionally, we investigated the effect of heparin $(200 \mathrm{u} / \mathrm{kg}$ bw i.v., $\mathrm{n}=12)$ on thrombus induction by $10 \% \mathrm{AlCl}_{3}$. During and after induction, arterial flow was measured for 30 min with a flow meter (Transonic TS420). Occlusion time $\left(t_{50}\right)$ was defined as the time when $50 \%$ reduction of the initial flow occurred.

For the in vivo MRI experiment, thrombi were induced with $10 \% \mathrm{FeCl}_{3}$ or $20 \% \mathrm{AlCl}_{3}$ as described before and, subsequently, coronal gradient echo MR images were acquired capturing both carotid arteries (TE/TR 10/750 ms, slice thickness $1 \mathrm{~mm}$ ). Afterwards, the right carotid artery was excised and examined with two-photon laser scanning microscopy (TPLSM) (Leica SP5 imaging platform). The vessel wall and thrombus were visualized using auto-fluorescence and presented as a Maximum Intensity Projection (MIP). 


\subsection{Results}

$T_{1}$ - and $T_{2}$-weighted images of the vials with $\mathrm{FeCl}_{3}$ and $\mathrm{AlCl}_{3}$ and the corresponding SNR in the vials are depicted in Figure 3.1 A-D. In both weightings, the SNR of the vials with $\mathrm{FeCl}_{3}$ strongly decreases for higher concentrations, while for $\mathrm{AlCl}_{3}$ it remains nearly constant. The $T_{1}$-weighted image of $\mathrm{FeCl}_{3}$ shows hyperintensities with respect to the surrounding water in the concentration range of $0.02-0.1 \%$. In the $T_{2}$-weighted images, the concentration of $0.02 \% \mathrm{FeCl}_{3}$ was hyperintense. In the $0.5-0.1 \%$ and 0.1 $1.0 \%$ concentrations range, hypointensities and susceptibility artifacts are observed in the $T_{1}$ - and $T_{2}$-weighted images, respectively. No artifacts were found for the vials filled with $\mathrm{AlCl}_{3}$.

In the in vivo carotid artery thrombosis experiments, the $\mathrm{t}_{50}$ was similar for $\mathrm{FeCl}_{3}$ and $\mathrm{AlCl}_{3}(543 \pm 205 \mathrm{~s}$ versus $432 \pm 206 \mathrm{~s}$, mean $\pm \mathrm{SD}$, ANOVA: NS), respectively. Heparin prolonged the $t_{50}$ for $\mathrm{AlCl}_{3}$ to $723 \pm 132 \mathrm{~s}(\mathrm{P}<0.05)$.

Figure $3.1 \mathrm{E}$ shows a coronal MR image of both carotid arteries after thrombus induction in the right carotid artery with $\mathrm{FeCl}_{3}$ (asterisk). The black spot is a typical susceptibility artifact that originates from residual $\mathrm{FeCl}_{3}$. The coronal MR image in Figure $3.1 \mathrm{~F}$ shows a thrombus which was induced with a high concentration (20\%) of $\mathrm{AlCl}_{3}$. The thrombus in the carotid artery can be discriminated from the surrounding tissue (arrow), and did not show any artifact. Figure $3.1 \mathrm{G}$ and $\mathrm{H}$ show TPLSM images of thrombi induced with $\mathrm{FeCl}_{3}$ and $\mathrm{AlCl}_{3}$, respectively. These images display normal and comparable morphology, indicative of similar thrombi. See also the additional histology of the thrombus and vessel wall in the appendix. 


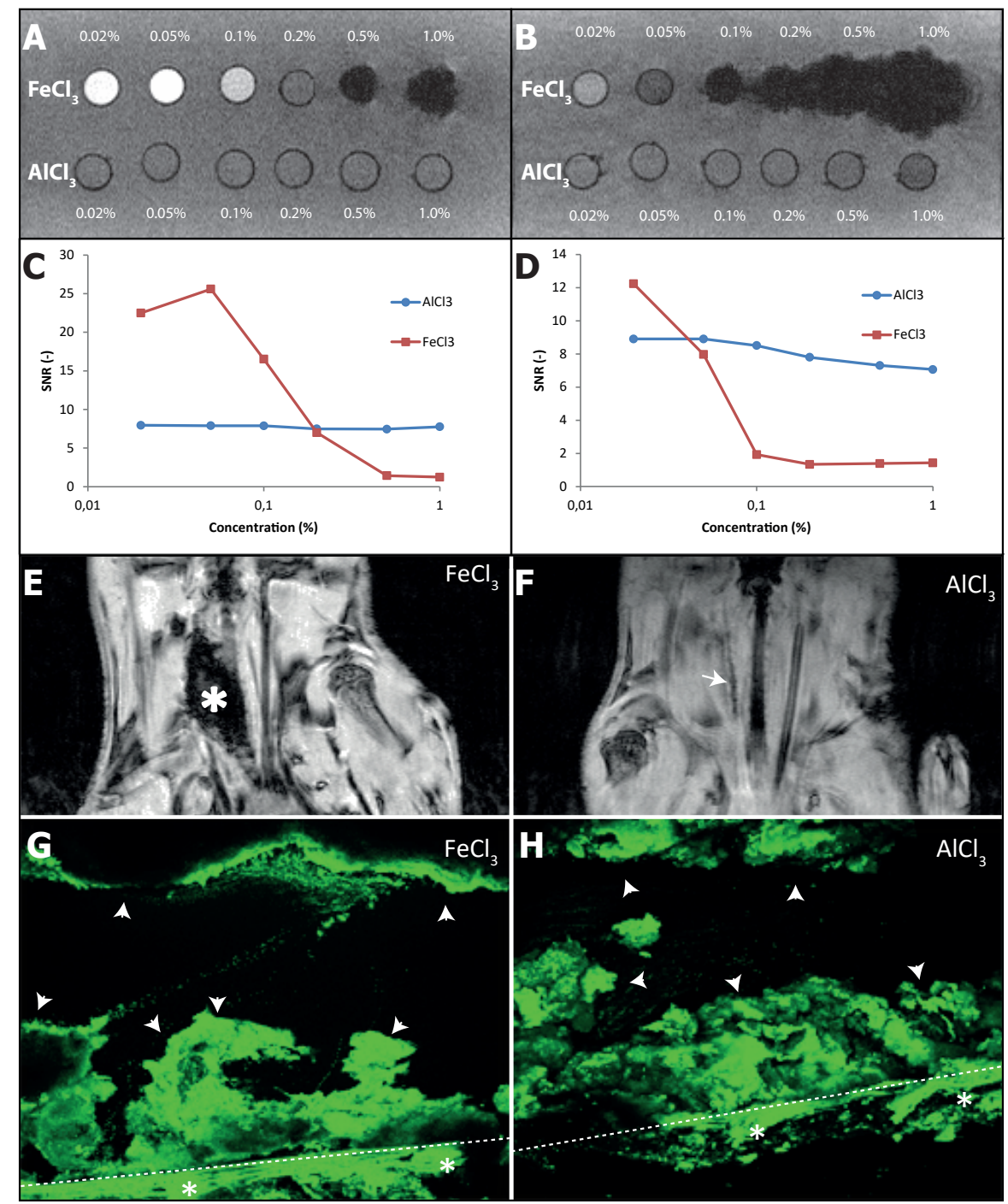

Figure 3.1 - $T_{1}$-weighted $(\mathrm{A})$ and $T_{2}$-weighted $(\mathrm{B})$ gradient echo MR images of vials filled with $\mathrm{FeCl}_{3}$ or $\mathrm{AlCl}_{3}$, respectively, in the range from $0.02 \%$ to $1 \%$. For $\mathrm{FeCl}_{3}$, strong intensity and distortion artifacts appear depending on the $\mathrm{FeCl}_{3}$ concentration and the MRI pulse sequence. No large intensity differences were observed for the vials containing $\mathrm{AlCl}_{3}$ relative to the surrounding water. The corresponding signal-to-noise ratio (SNR) decreases strongly as a function of concentration for $\mathrm{FeCl}_{3}$, but not for $\mathrm{AlCl}_{3}$ for both the $T_{7}$ - (C) and $T_{2}$-weighted (D) MR sequence. In E and F MR images are shown of the neck region of mice, including the carotid arteries. In both mice a thrombus was induced in the right carotid artery (RCA) using $\mathrm{FeCl}_{3}(\mathrm{E})$ or $\mathrm{AlCl}_{3}(\mathrm{~F})$. In panel $\mathrm{E}$, a large artifact (dark region indicated by asterisk), originating from residual $\mathrm{FeCl}_{3}$ severely affects thrombus-visualization at the RCA. In panel $\mathrm{F}$, a thrombus is visualized in the RCA (arrow) without this artifact. Panel $\mathrm{G}$ and $\mathrm{H}$ show two-photon laser scanning microscopy images of similar thrombi induced with $\mathrm{FeCl}_{3}(\mathrm{G})$ and $\mathrm{AlCl}_{3}(\mathrm{H})$ as auto-fluorescence Maximum Intensities Projections (MIP). In both images the thrombus is indicated with arrow heads, the vessel wall is indicated with an asterisk and delineated with a dotted line. 


\subsection{Conclusion and discussion}

The present study illustrates potential pitfalls when using $\mathrm{FeCl}_{3}$ for inducing thrombi and subsequent examination with MRI. Low concentrations of $\mathrm{FeCl}_{3}$ can already affect the MR signal by causing hypo- and hyperintensities in the vicinity of the thrombus. Thus, the interpretation of contrast-enhancing effects of a thrombus-targeted contrast agent becomes ambiguous as one cannot discern whether the contrast agent or the thrombus-inducing agent underlies the image contrast effect. Occlusion times $\left(t_{50}\right)$ were not significantly different for $\mathrm{FeCl}_{3}$ and $\mathrm{AlCl}_{3}$. As expected for a classical thrombus formation supported by coagulation, heparin significantly prolonged the occlusion time in the $\mathrm{AlCl}_{3}$ model similar to the reported effect in the $\mathrm{FeCl}_{3}$ model ${ }^{[9]}$. Even at high concentrations (20\%) in vivo MRI did not show any artifact. The use of $\mathrm{AlCl}_{3}$ may provide a solution in such MRI studies, and allows a better visual discrimination between the effects of the clot-inducing agent and imaging contrast agent. 


\section{Appendix, histological analysis}

An additional histological analysis of the $\mathrm{FeCl}_{3}$ - and $\mathrm{AlCl}_{3}$-induced thrombosed arteries was performed. After the occlusion experiment, all mice were euthanized with an i.p. injection of pentobarbital (100 mg/kg bw), the carotid artery was excised and fixed in $4 \%$ paraformaldehyde for 24 hours, and transferred in $70 \%$ ethanol. The tissue was dehydrated and permeabilized for paraffin perfusion. After paraffin perfusion, the tissues were blocked in paraffin and sections $(5 \mu \mathrm{m})$ were cut on a microtome (Leica Microsystems, Nussloch, Germany). Slides were examined on a microscope (Olympus BX51) equipped with a camera (Olympus XC10) after staining with hematoxylin/eosin (HE).

HE staining showed for $\mathrm{AlCl}_{3}$ and $\mathrm{FeCl}_{3}$ induced thrombi comparable platelet and erythrocyte composition in Figure $3.2 \mathrm{~A}\left(\mathrm{FeCl}_{3}\right)$ and Figure 3.2 B $\left(\mathrm{AlCl}_{3}\right)$. On the site where the thrombus was induced, the endothelial cells were damaged by both methods (panels $\mathrm{C}$ and $\mathrm{D}$ ), in contrast to the opposite site of induction (arrows in panels $\mathrm{E}$ and F). Furthermore, the HE staining showed dioxides of $\mathrm{FeCl}_{3}$ at the intersection of vessel wall and lumen where the thrombus was induced.

Figure 3.2 - (Page 53) Panels A and B show axial coupes of the carotid artery at the location where the filter paper for induction was applied. Both images show an occlusive thrombus for $\mathrm{FeCl}_{3}$ and $\mathrm{AlCl}_{3}$ and the thrombus composition of red and white thrombus is comparable. Panel C and D show a detailed view of the vessel wall where the thrombus was induced. Arrows indicate the absence of endothelial cells in both panels, whereas for $\mathrm{FeCl}_{3}$ also at this location oxides of $\mathrm{FeCl}_{3}$ were found (brown). In panel $\mathrm{E}$ and $\mathrm{F}$, the arrows indicate the presence of endothelial cells on the non-damaged site of the carotid artery. 


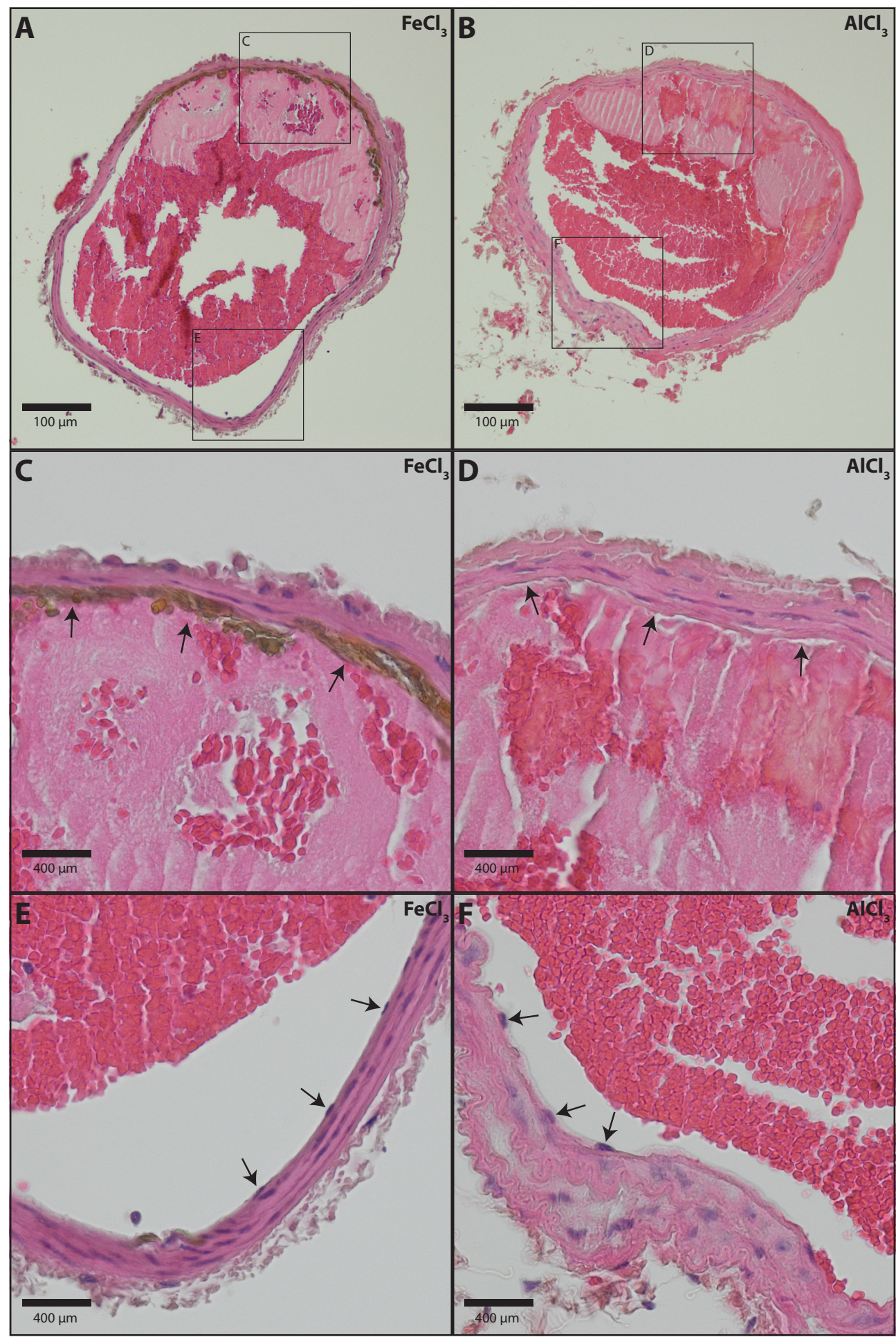




\section{References}

1. Ciesienski, K.L. and P. Caravan, Molecular MRI of Thrombosis. Curr Cardiovasc Imaging Rep. 4(1): p. 77-84.

2. Miserus, R.J., et al., Molecular MRI of early thrombus formation using a bimodal alpha2antiplasmin-based contrast agent. JACC Cardiovasc Imaging, 2009. 2(8): p. 987-96.

3. Botnar, R.M., et al., In vivo molecular imaging of acute and subacute thrombosis using a fibrin-binding magnetic resonance imaging contrast agent. Circulation, 2004. 109(16): p. 2023-9.

4. Duerschmied, D., et al., Molecular magnetic resonance imaging allows the detection of activated platelets in a new mouse model of coronary artery thrombosis. Invest Radiol. 46(10): p. 618-23.

5. von zur Muhlen, C., et al., Magnetic resonance imaging contrast agent targeted toward activated platelets allows in vivo detection of thrombosis and monitoring of thrombolysis. Circulation, 2008. 118(3): p. 258-267.

6. Ta, H.T., et al., Enzymatic Single-Chain Antibody Tagging A Universal Approach to Targeted Molecular Imaging and Cell Homing in Cardiovascular Disease. Circulation Research, 2011. 109(4): p. 365-U67.

7. McCarthy, J.R., et al., Multimodal Nanoagents for the Detection of Intravascular Thrombi. Bioconjugate Chemistry, 2009. 20(6): p. 1251-1255.

8. Westrick, R.J., M.E. Winn, and D.T. Eitzman, Murine models of vascular thrombosis (Eitzman series). Arteriosclerosis, thrombosis, and vascular biology, 2007. 27(10): p. 2079-93.

9. Wang, $\mathrm{X}$. and L. Xu, An optimized murine model of ferric chloride-induced arterial thrombosis for thrombosis research. Thrombosis research, 2005. 115(1-2): p. 95-100. 


\section{Clinical perspectives of hybrid}

proton-fluorine magnetic resonance imaging and

\section{spectroscopy}

M. Wolters, S.G. Mohades, T.M. Hackeng, M.J. Post, M.E. Kooi, W.H. Backes

Invest Radiol. 2013 May;48(5):341-50.

doi: 10.1097/RLI.0b013e318277528c

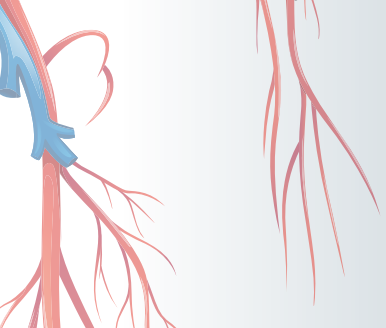


The number of applications of fluorine $\left({ }^{19} \mathrm{~F}\right) \mathrm{MR}$ imaging and spectroscopy in biomedical and clinical research is steadily growing. The $100 \%$ natural abundance of fluorine and its relatively high sensitivity for MR ( $83 \%$ to that of protons), makes it an interesting nucleus for a wide range of MR applications. Fluorinated contrast media have a number of advantages over conventionally used gadolinium or iron-based contrast agents. The absence of an endogenous fluorine background intensity in the human body facilitates reliable quantification of fluorinated contrast medium or drugs. Anatomy can be visualized separately with proton MR Imaging, creating the application of hybrid ${ }^{1} \mathrm{H} /{ }^{19} \mathrm{~F}$ MR imaging. The availability of two channels (i.e. the ${ }^{1} \mathrm{H}$ and ${ }^{19} \mathrm{~F}$ channel) enables dual target molecular imaging. Recently, novel developments have emerged on fluorine-based contrast media in pre-clinical studies and imaging techniques. The developments in fluorine MR seem promising for clinical applications, with contributions in therapy monitoring, assessment of lung function, angiography and molecular imaging. This review outlines the translation between recent advances in pre-clinical MR imaging and spectroscopy to future perspectives of clinical hybrid ${ }^{1} \mathrm{H} /{ }^{19} \mathrm{~F}$ MR imaging applications. 


\subsection{Introduction}

\section{Advantages of 19F-based contrast media}

Conventional Magnetic Resonance (MR) techniques are based on the detection of the signal from mobile protons $\left({ }^{1} \mathrm{H}\right)$ of water or lipids. Protons are highly abundant in the body and their concentration and magnetic properties vary with anatomy. These variations boost a background intensity that is inherently non-homogeneous. To enhance the contrast between a target tissue and the background, $T_{\text {- }}$-shortening (e.g. gadoliniumbased contrast media) or $T_{2}$-shortening (e.g. iron-based contrast media) contrast agents (CAs) are frequently utilized. However, intrinsic variations in the pre-existing background signal of the human body lead to an overall contrast that remains sub-optimal, especially at low concentrations of the CA. Furthermore, the relation between the signal intensity and gadolinium or iron concentration is essentially nonlinear and prone to artifacts such as magnetic field inhomogeneities, which make it difficult to uniquely translate a signal intensity change to the local contrast agent concentration ${ }^{[1]}$. For many of these problems fluorine MR imaging or spectroscopy would offer a solution. The normal physiological concentration of detectable (mobile) fluorine compounds in the human body is below the detection limit of regular fluorine MR techniques. Therefore, fluorine $\left({ }^{19} \mathrm{~F}\right)$ is measured as an exogenous contrast medium on the ${ }^{19} \mathrm{~F}$ channel separately from the proton anatomy signal on the ${ }^{1} \mathrm{H}$ channel, while $T_{1}$ en $T_{2}$ shortening contrast agents affect the signal intensity of endogeneous water and fat protons in tissue. Since the information from the ${ }^{19} \mathrm{~F}$ channel can be combined with the anatomical information from the ${ }^{1} \mathrm{H}$ channel, ${ }^{1} \mathrm{H} /{ }^{19} \mathrm{~F}$ MR imaging is a hybrid technique where the image contrast is not hindered by intrinsic variations in the (proton) tissue background intensity of the anatomy.

\section{Current status}

Clinical MR imaging of fluorine has been a research topic since the early days of MRI [2-4]. Preclinical research shows a broad number of applications ranging from non-invasive oximetry in tumors ${ }^{[5,6]}$, molecular MRI of blood clots ${ }^{[7,8]}$ and cell tracking ${ }^{[0]}$. Together with the fluorine MR techniques, novel contrast media have been developed in recent years. A number of these applications are already translated to clinical studies, of which the responses of colorectal metastasis to 5 -fluorouracil ( $5 \mathrm{FU}$ ) treatment are well known $[10,11]$. The use of fluorine contrast provides a number of distinguished advantages over gadolinium or iron-oxide enhanced MR examinations. The application of fluorine in MR imaging or spectroscopy (MRS) has already been discussed in several reviews $[9,12-18]$. These reviews describe in great detail the biomedical and pre-clinical research capabilities of fluorine MR. The present review focuses on the potential of fluorine imaging and spectroscopy applications as a hybrid ${ }^{1} \mathrm{H} /{ }^{19} \mathrm{~F}$ MR imaging technique in clinical studies. 


\subsection{Fluorine properties for MR}

\section{MR properties of fluorine}

Conventional gadolinium and iron based contrast agents increase the $r_{1}$ and $r_{2}$ relaxivities of protons, thereby enhancing the signal intensity of surrounding protons, while the contrast agent itself is not directly detected. In contrast, the fluorine acts as a direct contrast medium since the signal of the fluorine nucleus itself is acquired. To discern direct from indirect signal effects, we use the concept of contrast medium for fluorine components (direct signal) and contrast agent for conventional gadolinium or iron based compounds (indirect signa), respectively. The signal intensity linearly increases with fluorine concentration. Fluorine MR is thus limited by the amount of fluorine that can be administered. Other important physical MR properties for fluorine nuclei are (see Table 4.1): (i) the relative sensitivity $\left(83 \%\right.$ for ${ }^{19} \mathrm{~F}$ relative to $\left.{ }^{1} \mathrm{H}\right)$ which depends on the gyromagnetic ratio $\gamma$ and the natural abundance, (ii) the spectral line-width, which is quite narrow for fluorine compounds with a long $T_{2}$ relaxation time ${ }^{[19,20]}$ (see Figure 4.1), and (iii) the bandwidth of the chemical shift of the various peaks. These concepts are explained in more detail in Figure 4.2. The $100 \%$ natural abundance of fluorine, on earth it is found only as the stable isotope fluorine-19, and the high MR sensitivity of the nucleus, makes this atom highly advantageous for the detection of fluorine-based contrast media ${ }^{[21]}$.

\section{Administration of fluorine}

The exogenous administration of fluorine can be performed as a fluorinated drug or contrast medium in the form of liquids, gases or emulsions. Depending on its application for hybrid ${ }^{1} \mathrm{H} /{ }^{19} \mathrm{~F}$ MR imaging, the administration route of the fluorinated drug or contrast medium can be intravenously, orally, by inhalation or intratumorally. Fluorinated contrast media are usually hydrocarbons in which all hydrogen atoms are replaced by fluorine, forming perfluorocarbon (PFC) molecules. Perfluorocarbons, in liquid or gas form, are given as a contrast medium in several clinical imaging applications utilizing ultrasound, computed-tomography or MRI ${ }^{[27-30]}$. PFCs are almost insolvable in water and therefore fluorinated contrast media are often emulsified for the intravenous injection. Fluorine-loaded (nano) emulsions can be used for molecular imaging applications with sparse receptor concentrations. A high load of fluorine per nanoparticle then boosts the fluorine signal intensity.

\section{Biosafety and excretion}

Fluorine, incorporated in drugs, modifies the chemical properties, biological activity, biodistribution and/or the clearance routes of the original drug ${ }^{[31]}$. Fluorine increases the lipophilicity of the drug and hence the fat solubility, which results in passive diffusion of the drug into cell membranes and increases the intracellular bioavailability of the drug. In addition, chemically reactive metabolites can be blocked by including fluorine in the drug's molecular structure to reduce its toxicity. Almost one-fifth of the available pharmaceutical products today have at least one fluorine moiety in their structure ${ }^{[32]}$, which may provide the opportunity to study their biodistribution with hybrid ${ }^{1} \mathrm{H} /{ }^{19} \mathrm{~F}$ MR imaging and spectroscopy. The absence of fluorine in the background signal of the 
Table 4.1 - Properties of the most commonly used nuclei (spin $1 / 2$ ) for in vivo MR measurements [22]. The inherent MR sensitivity of nuclei depends on the gyromagnetic ratio (proportional to $\gamma^{3}$ ) and the relative natural abundance of the isotope ${ }^{[23]}$. The product of natural abundance and inherent sensitivity is high for ${ }^{1} \mathrm{H}\left(100 \%\right.$ by definition) and ${ }^{19} \mathrm{~F}(83 \%)$. Furthermore, the chemical shift range is broad for fluorine. These properties make fluorine attractive for MRI.

\begin{tabular}{|c|c|c|c|c|c|c|}
\hline Nucleus & $\begin{array}{c}\text { Natural } \\
\text { abundance } \\
(\%)\end{array}$ & $\gamma(\mathrm{MHz} / \mathrm{T})$ & $\begin{array}{l}\text { Average in vivo } \\
\text { concentration } \\
(\mathrm{mol} / \mathrm{L})\end{array}$ & $\begin{array}{l}\text { Relative NMR } \\
\text { sensitivity (\%)* }\end{array}$ & $\begin{array}{l}\text { Relative NMR } \\
\text { frequency }(\%)^{*}\end{array}$ & $\begin{array}{l}\text { Chemical Shift } \\
\text { range (ppm) }\end{array}$ \\
\hline${ }^{1} \mathrm{H}$ & 99.98 & 42.6 & 99 & 100 & 100 & $13(-1-12)$ \\
\hline${ }^{13} \mathrm{C}$ & 1.11 & 10.73 & 0.1 & $1.77 \cdot 10^{-2}$ & 25.1 & $200(0-200)$ \\
\hline${ }^{19} \mathrm{~F}$ & 100 & 40.06 & 0.0066 & 83 & 94.1 & $700(-300-400)$ \\
\hline${ }^{31} \mathrm{P}$ & 100 & 17.26 & 0.35 & 6.63 & 40.5 & $430(-180-250)$ \\
\hline
\end{tabular}

* Relative to ${ }^{1} \mathrm{H}$ expressed in percentage.

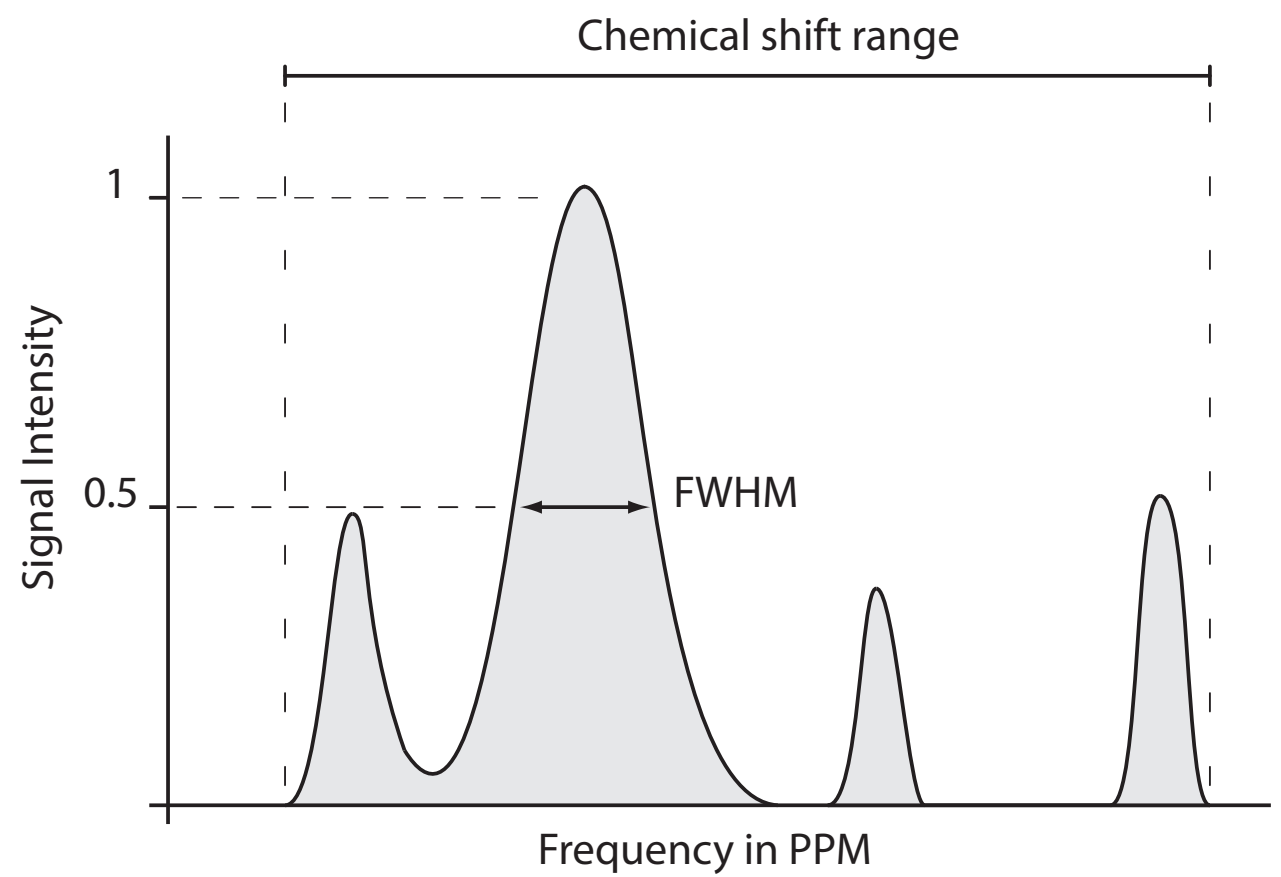

Figure 4.1 MR spectrum showing how the line-width of spectral peaks is characterized by the full-width-at-half-maximum (FWHM) and the definition of the chemical shift range. The spectral line-width is the broadness of a peak, given in parts per million (ppm) relative to the central frequency and is a property of the nucleus and its environment. It is usually expressed as the FWHM. The chemical shift range is also given in ppm and is the spectral range covering all the spectral peaks. Nuclei with spin $1 / 2$ (e.g. fluorine) generally have a small line-width due to a long transverse relaxation time $\left(T_{2}\right)$ resulting in a higher signal-to-noise-ratio and also a better distinction between different spectral peaks ${ }^{[24]}$. 


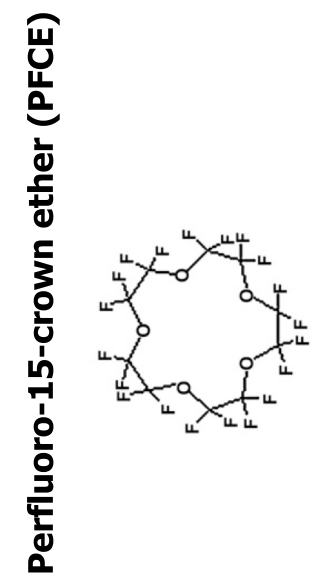

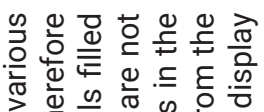

던 듕 등 은 인

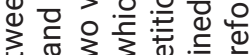

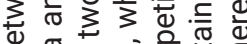

윤

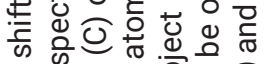

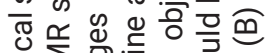

, $\sum$ ס

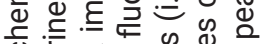

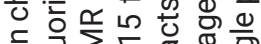

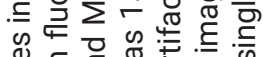

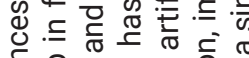

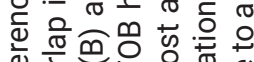

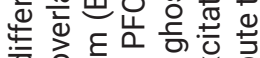

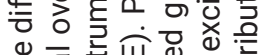

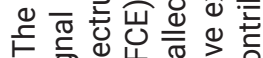

क क्ज़ की

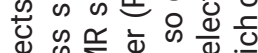

屯थ

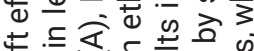

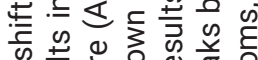

क

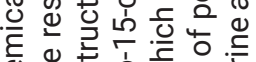

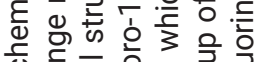

음 정 을 向은

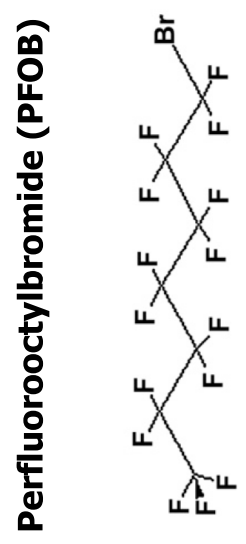

(4)

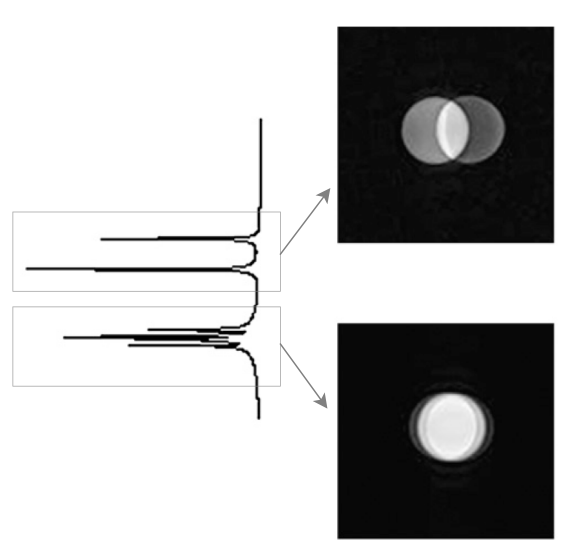

Frequency encoding 웡 क는

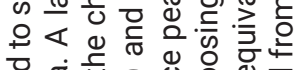
ซ 넌

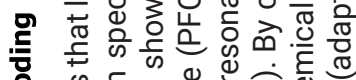

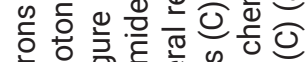

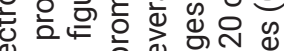
ब ब ब

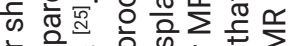

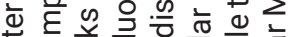

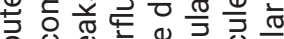
웡

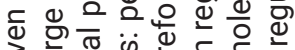
ญ

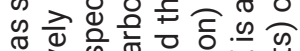

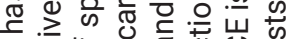
d 든 든 흥 늫늠 등

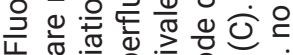

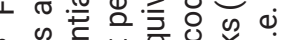
인

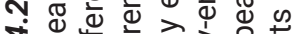

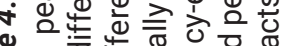

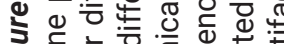
S.

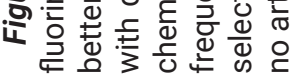


body results in a more reliable quantification of fluorine contrast media compared to gadolinium or iron-based agents ${ }^{[3,34]}$.

Pharmacokinetic characteristics are important for fluorine-loaded emulsions when applied as imaging contrast medium. The half-life in the blood and the reticuloendothelial system (RES) of PFC-loaded nanoparticles depends on the size and coating properties and is typically 8 to 24 hours in humans ${ }^{[3,36]}$. After introduction in the circulation, nanoparticles are taken up by the RES. This uptake depends on the dose and determines the plasma half-life. After the uptake, the nanoparticles slowly break down and subsequently the PFC molecules bind to the blood lipids, which facilitates the circulation. Due to absence of any in vivo metabolism, PFCs are mainly excreted through expiration via the lungs. The rate of this excretion depends on the vapor pressure of the PFCs. The half-life in the RES increases exponentially with increasing molecular weight ${ }^{[36,37]}$. PFC liquids are chemically and biologically inert due to the highly stable carbon-fluorine bonds. PFCs have been used in emulsions such as blood substitutes ${ }^{[38,39]}$. The first generation PFC-based blood substitute Fluosol, based on perfluorodecalin (FCD) and perfluorotripropylamine (FTPA), became commercially available in the early nineties. This product is not on the marked anymore, since the FDA approval of Fluosol was withdrawn due to the difficulty of frozen storage and subsequent thawing of the product ${ }^{[40]}$. The long-term biosafety of Fluosol was also questioned. Even one year after administration, PFCs were found in the lung tissue ${ }^{[41]}$. Clinical trials of a second generation blood substitute Oxygent, based on perfluorooctylbromide (PFOB), showed some adverse, but reversal, clinical effects such as headache, backache and flu-like symptoms ${ }^{[42]}$. The Phase III clinical studies of Oxygent, using PFOB, were suspended in $2001^{[43]}$, however the development of this product is resumed ${ }^{[44]}$. The PFCs used in these blood substitutes are considered safe, although some PFCs demonstrate a long-term retention in the RES ${ }^{[42,45]}$.

\section{Fluorine Signal Detection limit}

One of the most challenging factors of fluorine imaging or spectroscopy is the detection limit of the fluorine signal. Endogenous fluorine is found only in the bones and teeth where it has a very short $T_{2}$ relaxation time $(<100 \mu \mathrm{s})$ due its immobility ${ }^{[46]}$. Therefore, it is usually undetectable by common in vivo MR systems. On the other hand, contrast media have preferably a long $T_{2}$ relaxation time and are therefore easier to detect. However, the fluorine concentration of the contrast medium must be higher than the signal detection limit. This mechanism is basically different from paramagnetic and superparamagnetic contrast agents where relatively low concentrations already have an effect on the relaxivity of water. Note that the in vivo concentration for water protons $(99 \mathrm{~mol} / \mathrm{L})$, is often far above the signal detection limit in contrast to fluorine. For fluorine the administered concentration is in the order of a factor 104-106 lower than the in vivo proton concentration of water. The lowest concentration of a fluorine compound that can be detected depends not only on the acquisition parameters, e.g. pulse sequence (preferably with a long TR and short TE), voxel volume, etc., and also on the type of fluorine contrast medium. In general, compounds with chemically equivalent fluorine atoms have a lower detection limit than compounds with the same number of chemically nonequivalent fluorine atoms. When the fluorine atoms 
are chemically equivalent, they contribute to the same spectral peak (Figure 4.2). Otherwise, the signal is, in fact, distributed over multiple, smaller, spectral peaks, which reduces the signal-to-noise (SNR) ratio and gives rise to chemical shift artifacts. Klomp et al demonstrated quantitative measurements of $5 \mathrm{FU}$ in patients treated for colorectal cancer with oral capecitabine ${ }^{[47]}$. They were able to measure absolute levels of capecitabine metabolites in the liver with spectroscopic imaging of $29 \mu \mathrm{mol} / \mathrm{kg}$

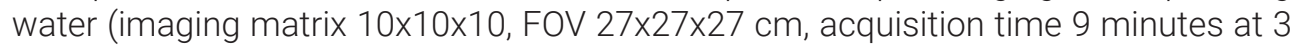
Tesla. In studies of Bolo et al serotonin reuptake inhibitors (SRRI's) were measured in the brain and bone marrow. They measured in vivo fluorine levels ranging from 13 to $47 \mu \mathrm{mol} / \mathrm{L}$ in 17 minutes at 3 Tesla ${ }^{[48,49]}$. Schneider et al measured, in a study where patients received a fluorine containing antihistamine, fluorine levels of $20 \mu \mathrm{mol} / \mathrm{L}$ at 4 Tesla ${ }^{[50]}$. These studies show that the typical measurable concentration are tens of micromolars in a clinically realizable acquisition time. 


\subsection{Emerging clinical fluorine MR applications}

In daily clinical practice, MR images are acquired by the proton channel and provide, in general, $T_{1}$ or $T_{2}$ weighted image contrast. This contrast is defined by the $T_{1}$ and $T_{2}$ relaxation times of the tissue and can be enhanced by contrast agents. Due to the absence of fluorine in tissue, hybrid ${ }^{1} \mathrm{H} /{ }^{19} \mathrm{~F}$ MR imaging and spectroscopy requires other imaging strategies than conventional proton imaging. Hybrid ${ }^{1} \mathrm{H} /{ }^{19} \mathrm{~F} M \mathrm{M}$ aims to visualize the spatial differences in fluorine nuclei density of fluorine contrast media or fluorine containing drugs. For successful fluorine detection, the typically long $T_{1}$ relaxation time of fluorine compounds and various spectral frequencies of fluorine have to be considered carefully. Since it is the fluorine density, rather than relaxation time, that creates the contrast in a fluorine image, this technique benefits highly from new developments in nanoparticle design and MRI hardware. Higher field MRI systems are becoming more common and the SNR increases with the increasing magnetic field ${ }^{[51]}$. Furthermore, increasing the fluorine load in contrast media increases the fluorine density and lowers the detection limit for fluorine particles, thus enabling high sensitivity imaging. These developments support the clinical translation of hybrid ${ }^{1} \mathrm{H} /{ }^{19} \mathrm{~F}$ MR imaging and spectroscopy with fluorine contrast media ${ }^{[12-18]}$.

\section{Drug tracking and therapy monitoring}

Chemotherapy with 5FU is widely used for the treatment of several cancers. However, the treatment response to 5FU therapy is highly variable. Despite changes in the general treatment regimes and combinations with other types of chemotherapies over the past 20 years, only $40-50 \%$ of the patients show a positive response ${ }^{[10]}$.

To improve this type of treatment, the therapy needs to be optimized to the individual patient. Therapy monitoring possibilities are required that allow the in vivo detection of fluorine to measure the bio-availability of the drug. The fluorine atom in 5FU allows measurement of its half-life with fluorine spectroscopy so that the treatment can be individualized on a patient-by-patient basis. Monitoring the in vivo distribution of a drug in general, during treatment of a patient, can answer fundamental questions about its interaction with the target and other tissues ${ }^{[52,53]}$. In the case where no local accumulation of the drug is observed, the treatment can be stopped to prevent adverse side effects of an otherwise ineffective treatment. Not only 5FU contains fluorine, but one fifth of the drugs already contain a fluorine atom including antidepressants, anti-inflammatory agents, anti-malaria drugs, antiviral agents, steroids and general anesthetics ${ }^{[32]}$. Table 4.2 provides an overview of human drug tracking studies. The fluorine in drugs is usually not added for imaging purposes, but to improve pharmacodynamic or pharmacokinetic behavior. A single fluorine atom might already alter the specific in vivo behavior of the drug and makes it possible to monitor these drugs directly. The antipsychotic drug haloperidol also has a fluorine atom incorporated. The pharmacokinetics of haloperidol were previously studied in patients who received an intra-muscular injection. The drug's fluorine signal could be measured with fluorine spectroscopy for at least 16 days, which was not possible with radioactive tracers ${ }^{[54]}$. Therapy monitoring with radioactive tracers provides high 


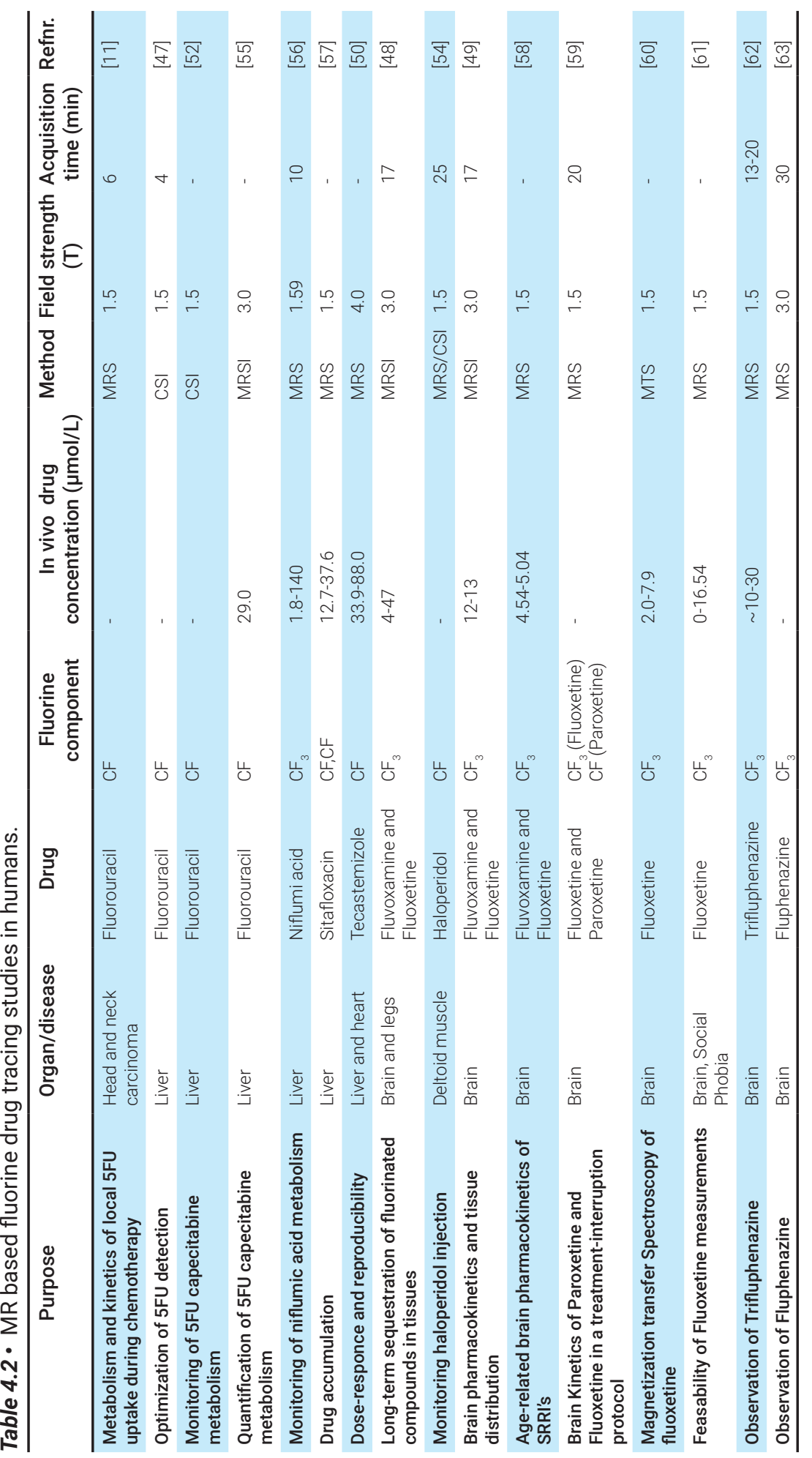


sensitivity, however, imaging time is limited due to the decay of the isotope and it causes radioactive exposure to the patient.

\section{Imaging fluorine containing drugs}

The in vivo concentration of a fluorine containing drug can be quite low making drug tracking challenging. In most cases, a single fluorine atom is incorporated in a molecule, which results in a single resonance peak and therefore does not suffer from chemical shift artifacts. For fluorine compounds that exhibit a single resonance peak, conventional spin or gradient echo pulse sequences are most suitable. When multiple resonance peaks arise from a compound with multiple (chemically in-equivalent) fluorine atoms, chemical shift artifacts will appear (Figure 4.2). The signal from each fluorine nucleus at the same location will not be positioned at the same pixel but will appear shifted with respect to other fluorine nuclei (i.e. chemical shift artifacts). Such chemical shift artifacts can be avoided with selective excitation or reception in traditional 2D or 3D gradient echo or spin echo acquisitions. With these techniques only a single fluorine nucleus, a CF3 or specific CF2 group will contribute to the image by using a selective RF excitation pulse with a narrow bandwidth, or a receiver with a narrow bandwidth ${ }^{20]}$. For such spectrally selective techniques, prior knowledge of the drug (or contrast media) is necessary to tune the pulse sequence.

Another cause of chemical shifts can arise from the metabolization of drugs. For example, the active metabolites of 5FU, cytotoxic fluoro nucleosides, have shifted resonance frequencies with respect to the original 5FU signal ${ }^{[11]}$. For the tracking

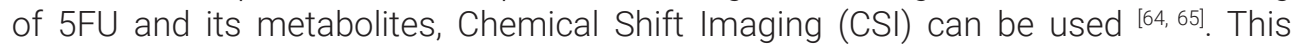
technique is suitable to prevent chemical shift artifacts in the MR images since it does not use frequency-encoding for the $x$-direction but phase encoding for both the $x$ - and y-direction. During the acquisition, CSI measures a spectrum for each voxel. One specific peak or several peaks from these spectra can be integrated or fitted to represent the voxel intensity of a single or multiple peaks. A disadvantage of CSI is that high spatial resolution scans are rather lengthy in time ${ }^{[66]}$. However, all spectral peaks can contribute to the image and the acquisition is not hampered by chemical shift artifacts.

\section{Tumor hypoxia imaging}

Tumor hypoxia is associated with resistance to radiation therapy and impaired delivery of chemotherapeutic agents. Measuring and monitoring of oxygen levels in tumors is usually an invasive procedure using needle shaped probes which only measure the partial oxygen pressure $\left(\mathrm{pO}_{2}\right)$ in a small localized sample volume. PFCs have a high oxygen carrying capacity and their $T_{1}$ relaxation time changes with the amount of oxygen bound ${ }^{[36]}$. Fluorine MRI with a PFC contrast medium represents a minimally invasive technique to map the oxygen level over the tumor. This has already been shown in several animal studies where the fluorine contrast medium was administered directly in the tumor $[6,19,64,67]$. In a recent study by Magat et al, spatial and temporal changes in tumor oxygenation could be observed which could lead to a better understanding of tumor hypoxia and improved treatment planning. In animal studies, PFCs such as perfluoropolyether (PFPE), perfluoro-15-Crown-5- 
ether (PFCE) and perfluorooctylbromide (PFOB) have been used successfully and can probably be translated to clinical contrast media for ${ }^{1} \mathrm{H} /{ }^{19} \mathrm{~F}$ MR imaging ${ }^{[5,8,68-72]}$. These PFC liquids have a high fluorine load and are chemically and biologically inert. The biological inertness is also increased by their hydrophobic and lipophobic properties which avert them from easy incorporation in tissues ${ }^{[36]}$.

\section{Lung ventilation imaging}

For the imaging of lungs, MRI is usually not the preferred imaging modality since the lungs have a low proton density. However, to detect ventilation abnormalities in lungs, the air can be replaced by a gas that can be detected with MR techniques. In current clinical research, hyperpolarized gases (e.g. ${ }^{3} \mathrm{He}$ or ${ }^{129} \mathrm{Xe}$ ) are frequently used to examine pulmonary volumes with MRI [73-75]. These gases are safe to use and obtain images with much higher SNR compared to fluorine gases, however, the production of hyperpolarized gases is expensive and requires dedicated equipment. Perfluorinated gases for ${ }^{19} \mathrm{~F}$ MR imaging may provide a cheaper alternative for hyperpolarized gases. They are ready to use, whereas hyperpolarized gases require preparation time for the hyperpolarization just before the examination. Furthermore, fluorine gases can be mixed with air, with only a small reduction in spin density.

In animal experiments, a number of compounds (e.g. $\mathrm{SF}_{6}, \mathrm{C}_{2} \mathrm{~F}_{6}, \mathrm{C}_{4} \mathrm{~F}_{4}, \mathrm{C}_{3} \mathrm{HF}_{7}$ and $\mathrm{CF}_{4}$ ) have been used to perform lung ventilation imaging [76-79]. Figure 4.3 shows lung images of a pig, breathing $30 \% \mathrm{C}_{2} \mathrm{~F}_{6}$ gas ${ }^{[7]}$. In clinical research, successful results were presented with the $\mathrm{SF}_{6}$ gas ${ }^{[80}$. Fluorinated gases have very short relaxation times (e.g. $T_{1}$ of $1.65 \mathrm{~ms}$ for $\mathrm{SF}_{6}$ and $7.9 \mathrm{~ms}$ for $\mathrm{C}_{2} \mathrm{~F}_{6}$ ). For lung ventilation imaging, such a short $T_{1}$ is an advantage as the longitudinal magnetization recovers rapidly and therefore a high number of images can be acquired per time unit. This is especially beneficial for the acquisition of many repetitions (averages) boosting the signal-tonoise ratio of the image, or to perform a dynamic acquisition to determine the wash-in and wash-out characteristics of the fluorinated contrast medium.

\section{Angiography}

MRI offers many different methods for angiography, with or without use of a contrast agent. Angiography without a contrast agent (e.g. time-of-flight or phase-contrastangiography) requires prior knowledge of the flow and a good background tissue suppression method ${ }^{[81]}$. For gadolinium contrast-enhanced angiography, fat tissue can be difficult to distinguish from contrast-enhanced blood vessels. Also a high level of background signal can be present due to the fast extravasation of the low-molecular weight contrast agents, which can be avoided only by fast image acquisition ${ }^{[7]}$. Fluorineenhanced hybrid ${ }^{1} \mathrm{H} /{ }^{19} \mathrm{~F}$ MR angiography may overcome these problems. Van Heeswijk et al recently demonstrated fluorine-enhanced hybrid ${ }^{1} \mathrm{H} /{ }^{19} \mathrm{~F}$ MR angiography in mice with a fluorine emulsion on an experimental 9.4 Tesla system. Neubauer et al showed that fluorine-enhanced angiography of rabbits is feasible on a clinical 1.5 Tesla MR System (Figure 4.4) [71,82]. Separate imaging of the fluorine contrast medium allows an overlay on the proton-based anatomical image. Due to the fluorine concentration, the spatial resolution is usually lower compared to proton based angiography. On higher field systems the spatial resolution can be further increased. In theory, increasing the 

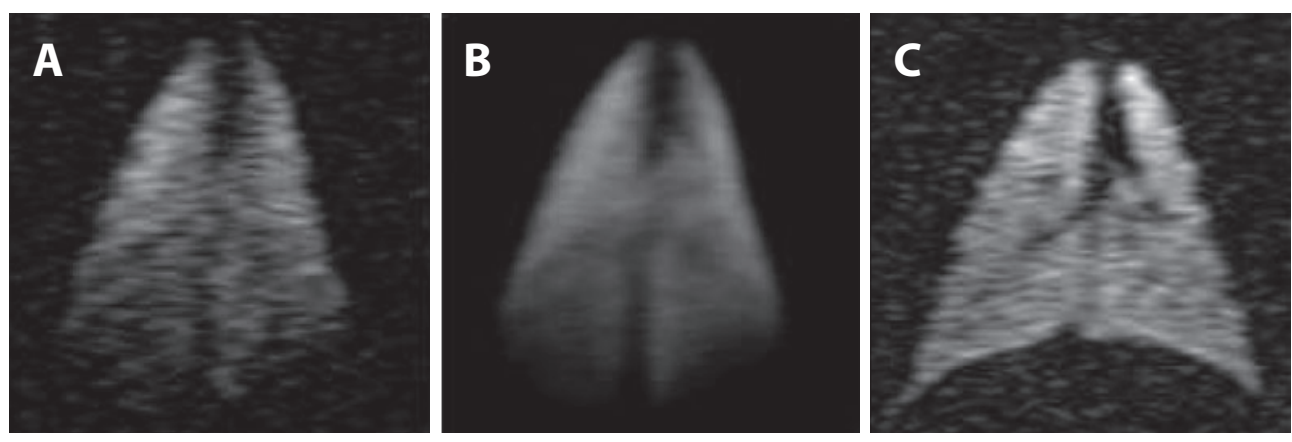

Figure 4.3 - Coronal lung images of a pig ventilated with $30 \% \mathrm{C}_{2} \mathrm{~F}_{6}$ gas. A and B) Nonslice selective images (i.e. projection images) with a $6.3 \times 3.1 \mathrm{~mm}^{2}$ resolution, image $A$ was obtained in 260 ms with a SNR of 7.9 and B in 26 seconds with an SNR of 63. C) Post mortem $3 \mathrm{D}$ acquisition with a $6.3 \times 3.1 \times 8.0 \mathrm{~mm}^{3}$ voxel size obtained in 10 minutes (adapted from ${ }^{[77]}$ ).
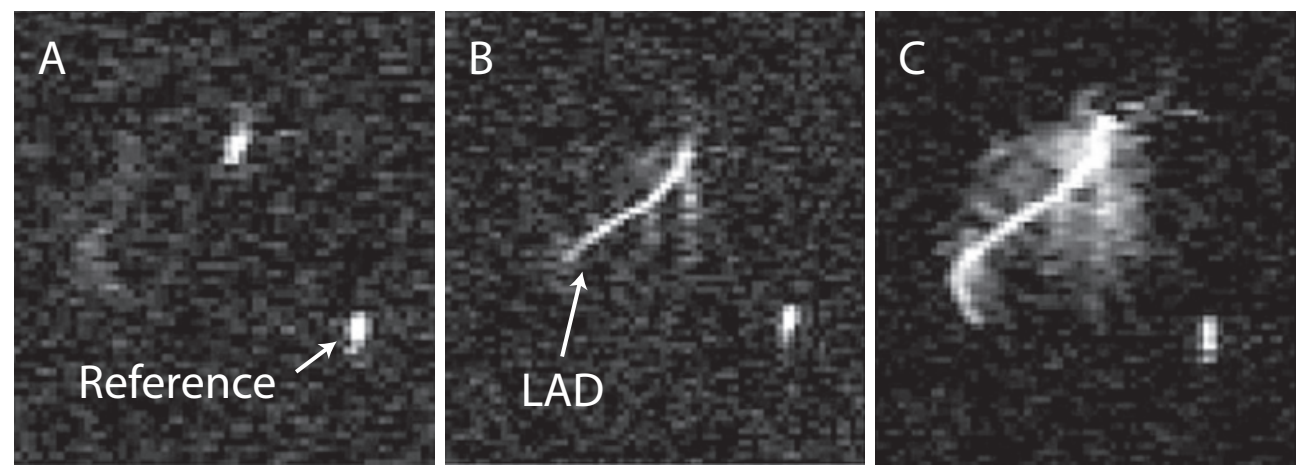

Figure 4.4 - Ex vivo fluorine angiography of a pig heart. PFCE nanoparticles were injected in the left anterior descending artery and dynamic ${ }^{19} \mathrm{~F}$ images were acquired with a $1.4 \times 2.0 \times 70$ $\mathrm{mm}^{3}$ resolution and a temporal resolution of 1.8 seconds. Panels $A, B$ and $C$ represents time points $0,7.2$ and 10.8 seconds after fluorine injection, respectively (adapted from ${ }^{[71]}$ ).

field strength from 1.5 to 7.0 Tesla approximately allows a reduction in pixel size by a factor of 2.1 and for a 2D acquisition by a factor of 1.7 for a 3D acquisition with preserved SNR. Higher field strengths are also beneficial for gadolinium contrast enhanced acquisitions, however, contrast enhancement of fluorine contrast media improves more at higher field strengths compared to gadolinium. The gadolinium contrast agent enhances the signal of water protons by increasing the $T_{1}$ relaxation. However, the $r_{1}$ relaxivity decreases at higher field strength. Fluorine-based contrast media are directly detected. The direct detection of fluorine contrast media leads to a linear relation between the signal intensity and the field strength.

To obtain a long plasma half-life of the fluorine emulsions for fluorine-enhanced hybrid ${ }^{1} \mathrm{H} /{ }^{19} \mathrm{~F}$ MR angiography, a particle size of $100-200 \mathrm{~nm}$ is preferred. This size prevents extravasation of the contrast medium, especially when the outer shell of the nanoparticles is "pegylated". Pegylation is a technique where poly-ethylene-glycol 
(PEG) tails are attached to the shell of the particle. Pegylation reduces the uptake of nanoparticles in the RES and therefore increases the plasma half-life ${ }^{[83-87]}$.

A disadvantage of the direct detection of fluorine contrast media is that a relatively high dose which is required. Van Heeswijk et al ${ }^{[82]}$, however, extrapolated the contrast medium dose that was administrated for animal ${ }^{19} \mathrm{~F}$ MR angiography to calculate the required dose for human studies, assuming the same experimental conditions. Their calculations resulted in a dose of $38 \mathrm{mM}$ PFC for human angiography studies, which was already previously administered in humans as blood substitute ${ }^{[88]}$.

\section{Molecular MRI with fluorine}

With molecular MRI the expression of specific biomarkers (e.g. protease or protein kinases) or biological processes (e.g. apoptosis or angiogenesis) can be visualized [89-93]. The great advantage of MRI is that versatile types of imaging, including anatomy and functional (e.g. flow, perfusion, activation, etc), can be combined and spatially matched to the expression of molecular biomarkers. In, for example, oncology, structural MRI provides information about tumor size and location, whereas molecular imaging can support the diagnosis with the status of angiogenesis and therefore the malignancy of a tumor $[94,95]$. The unique concept of molecular imaging is to target specific molecules by ligands attached to a contrast agent or medium, employing gadolinium, iron oxide or fluorine. Although certain biomarkers of biological processes are over-expressed, their concentration remain very low ${ }^{[96]}$. Therefore, nanoparticles with a large number of fluorine or gadolinium atoms per particle need to be used to induce sufficient signal per particle ${ }^{[97,98]}$ and to overcome the detection limit. Hybrid ${ }^{1} \mathrm{H} /{ }^{19} \mathrm{~F}$ Molecular MR imaging, utilizing fluorine is an emerging application and has important advantages compared to molecular MRI with gadolinium or iron oxide contrast agents. Low concentrations of gadolinium or iron oxides can be difficult to discern from a spatially inhomogeneous background signal. In a separate fluorine MRI measurement, the fluorine distribution can be related to the local anatomy ${ }^{[70}$, 99]. Caruthers et al demonstrated the in vitro targeting of thrombi with antibodies coupled to fluorine-loaded nanoparticles [100]. This experiment showed that two different fluorine components could be distinguished with selective excitation. Figure 4.5 shows an example of in vivo selective excitation [101], in which two populations of cells where labeled with PFOB or PFCE and could be visualized separately. For this approach it is necessary that the spectral frequencies are sufficiently separated. To minimize any spatial mismatches in hybrid ${ }^{1} \mathrm{H} /{ }^{19} \mathrm{~F}$ MR imaging between proton and fluorine MRI, both images can be acquired simultaneously [102].

\section{Distinction between bound and freely circulating nanoparticles}

Detection of angiogenesis in tumors has in recent years been widely explored with molecular MRI, by targeting biomarkers such as $a_{v} \beta_{3^{\prime}} a_{5} \beta_{1}$ or CD13 ${ }^{[06,103]}$. The presence of angiogenesis in a tumor increases the risk for further growth and formation of metastases, which classifies the tumor as malignant [104]. A general problem with MRI, is that a high dose of contrast agent or medium is often necessary to obtain sufficient accumulation at the site of interest. For reasons of limited spatial resolution, the targeting of activated endothelial cells (as in angiogenesis) cannot discern bound 
particles from particles that circulate in the adjacent blood space. Moreover, a low clearance rate will reduce the target-to-background ratio. A solution to this problem was provided by diffusion weighted fluorine spectroscopy.

Using a diffusion preparation step in the MRI pulse sequence, the signal of freely moving particles loses phase coherency and will therefore be suppressed. In this way, only bound particles are detected and it becomes possible to investigate the efficacy of the targeting agent more specifically. Waters et al have successfully demonstrated such a diffusion weighted fluorine spectroscopy sequence to selectively detect the binding of angiogenesis-targeted nanoparticles in transgenic mice with an upregulated neovasculature ${ }^{[68]}$.

\section{Cell labeling}

Cellular therapy is an emerging application for a wide range of diseases e.g. myocardial infarction [105, 106], neurodegenerative disease [107, 108], and cancer therapy [109]. For a successful clinical use of cellular therapies, a non-invasive imaging modality must be available to track and study the transplanted cells ${ }^{[110]}$. A number of pre-clinical studies already showed that ex vivo fluorine labeled stem cells and immunology cells, could successfully be tracked in vivo ${ }^{[9,101,111]}$. Partlow et al demonstrated the injection of PFOB and CE labeled stem/progenitor cells [101]. They were able to visualize both population cells separately with selective excitation (Figure 4.5). Fluorine nanoparticles are ex vivo internalized by the stem cells or immunology cells and subsequently administered. Hybrid ${ }^{1} \mathrm{H} /{ }^{19} \mathrm{~F}$ MR cell tracking technique provides the ability to quantify the number of cells and track them over time ${ }^{[9]}$. The absence of fluorine in the background signal of the body results in a more reliable quantification of fluorine contrast media in hybrid ${ }^{1} \mathrm{H} /{ }^{19} \mathrm{~F}$ applications compared to gadolinium or iron-based agents ${ }^{[33,34]}$.

Labeling of cells can also be applied in vivo. Fluorine nanoparticles are easily phagocytized by monocytes and macrophages due to their size [112]. Phagocytized nanoparticles will accumulate in the RES and in inflammatory areas [18]. The accumulation in inflammatory areas was for example used to study graft rejection in transplanted heart in mice to visualize the host innate immune response ${ }^{[113]}$. Fluorine loaded nanoparticles can be used to track such accumulations of phagocytes. Imaging of fluorine loaded nanoparticles to visualize acute inflammatory response in mice with a cardiac infarction is shown in Figure 4.6. In this study, the left anterior descending artery (LAD) was ligated and fluorine-loaded nanoparticles could be visualized with MRI in the infarct region ${ }^{[70]}$.

\section{Dual target hybrid 1H/19F MR imaging}

Noninvasive molecular imaging is usually based on the visualization of a single molecular biomarker. However, pathology and pathogenesis can generally be complex and the molecular signature of a disease may not be easily defined by one single biomarker. Techniques that would enable detection of multiple molecular biomarkers could therefore further improve the diagnosis on the basis of more complex molecular information. With fluorine-based contrast medium for hybrid ${ }^{1} \mathrm{H} /{ }^{19} \mathrm{~F} \mathrm{MR}$ imaging the use of multiple targeted imaging becomes within reach. For dual targeted hybrid ${ }^{1} \mathrm{H} /{ }^{19} \mathrm{~F}$ 


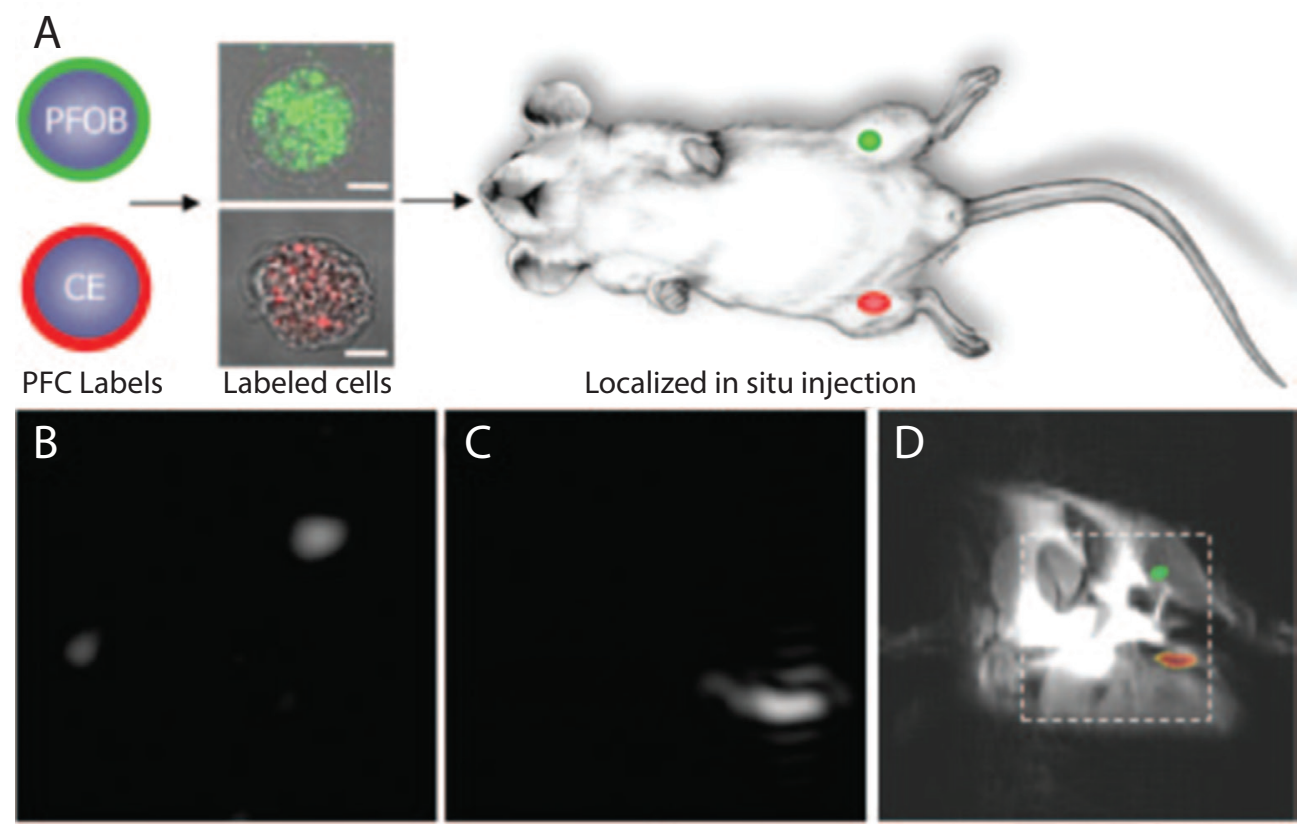

Figure 4.5 - Visualization and localization of labeled cells after in situ injection. Panel A, stem cells labeled with either PFOB (green) or CE (red), nanoparticles were locally injected into mouse thigh skeletal muscle. Panel $B$ and $C$, selective excitation permits imaging the fluorine signal attributable of PFOB-loaded (B) or CE-loaded cells (C) individually. Panel D shows an overlay of panel $B$ and $C$ onto a conventional ${ }^{1} \mathrm{H}$ image. This shows PFOB and $C E$ labeled cells localized to the left and right leg, respectively (dashed line indicates $3 \times 3 \mathrm{~cm}^{2}$ field of view for ${ }^{19} \mathrm{~F}$ images, adapted from ${ }^{[101]}$ ).

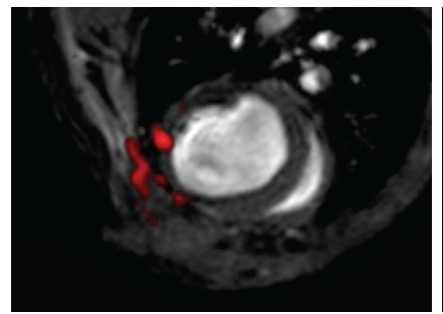

$1 d$ post OP

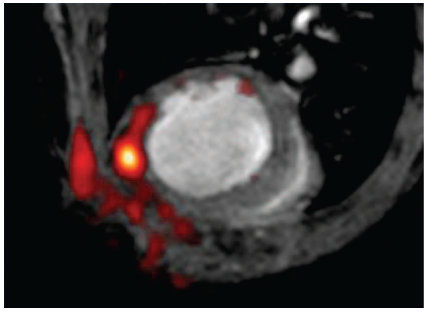

$3 d$ post OP

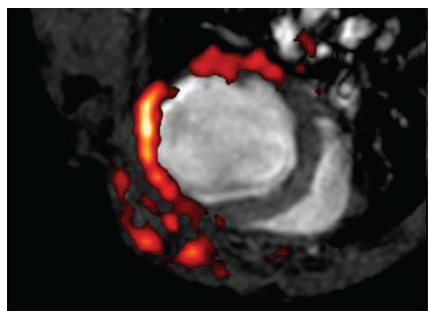

6d post OP

Figure 4.6 Short axis cardiac images of a mouse with a myocardial infarction, which was provoked by ligation of the LAD. After the ligation, PFCE nanoparticles were administered. A series of 3 images is shown, 1,3 and 6 days post-surgery, respectively. Fluorine images were depicted as a colored overlay on the anatomical images and show the accumulation of fluorine in the injured areas of the heart and the chest which was affected by the thoracotomy (adapted from ${ }^{[70]}$ ). 
MR imaging a combination of targeted fluorine nanoparticles and targeted gadolinium contrast agent, or two different nanoparticles with different spectral fluorine peaks could be used in one examination. Both contrast media can be separately imaged with selective excitation, as explained in Figure 4.5, or the whole spectrum can be acquired with CSI and separated afterwards by imaging post-processing techniques ${ }^{[101,114]}$. In the clinic, dual target imaging is currently only feasible by Single-Photon-EmissionComputer-Tomography (SPECT) and relies on the emission of energetically different gamma photons by different radioactive isotopes. Dual-isotope studies were used to visualize serotonin and dopamine distributions with myocardial perfusion scintigraphy or in the discrimination of early Parkinson disease ${ }^{[115-117]}$. Future applications of dual targeted MRI could be diagnostic imaging of deep vein thrombosis or thrombi from ruptured atherosclerotic plaques. Dual targeted hybrid ${ }^{1} \mathrm{H} /{ }^{19} \mathrm{~F} \mathrm{MR}$ imaging is able to reveal the location of the thrombus with fibrin targeted fluorine nanoparticles ${ }^{[8]}$, and to determine whether thrombolytic therapy will be effective or not. The sensitivity of a thrombus to thrombolytic therapy can possibly be evaluated with an a2-antiplasmin targeted gadolinium contrast agent that binds only to fresh thrombi ${ }^{[18]}$. Moreover, the contrast media for dual targeted MRI mentioned here, do not hamper by radioactive decay. This advantage makes relative long and reparative examinations possible.

\section{Clinical translation}

Human hybrid ${ }^{1} \mathrm{H} /{ }^{19} \mathrm{~F}$ MR imaging and spectroscopy is currently limited to drug tracking applications as shown in Table 4.2. Although these applications show that the detection of fluorine is possible in the range of tens of micromolars within clinically acceptable measuring time. This detection limit is quite high in comparison to PET where typical tracer concentrations can be as low as in the picomolar range ${ }^{\text {[53] }}$. Other fluorine MR applications depend on fluorine contrast medium, which is not available. Furthermore, hybrid ${ }^{1} \mathrm{H} /{ }^{19} \mathrm{~F}$ MR imaging in clinical practice requires dedicated MR equipment, such as broad-band RF amplifiers and coils, and approved contrast media. Unfortunately, currently not all vendors of clinical MR systems provide hardware and software options for fluorine applications. However, recent hardware developments as mostly available in research settings support the application of fluorine MR. High field systems, parallel imaging techniques or novel reconstruction methods (e.g. Compressed Sensing or Highly Constrained Back Projection) can further decrease the detection limit or reduce the acquisition time ${ }^{[119,120]}$. As most fluorine applications rely on fluorine density measurements and since some fluorine compounds have a short transverse $\left(T_{2}\right)$ relaxation, they will benefit from Ultra-short Echo Time (UTE) or Zero Echo time (ZTE) pulse sequences to increase the sensitivity of fluorine-MR [121]. However, these sequences are prone to chemical shift artifacts and should therefore preferentially be used for fluorine components with a single resonance peak to avoid these artifacts. For components with multiple resonance peaks alternative sequences such as Ultrashort Echo Time Spectroscopic Imaging (UTESI) can be used to obtain images and spectral information without chemical shift artifacts ${ }^{[122]}$.

Fluorine emulsions have shown good results in cell labeling, molecular imaging and angiography. Some fluorine emulsions already had FDA approval as blood substitutes and can probably be used as an MRI contrast medium ${ }^{[42]}$. Targeted fluorinated 
contrast media are also not yet available for clinical use. Molecular imaging is still a relative young discipline and the development and approval of contrast media can take more than a decade. Despite the many challenges, including the detection limit of fluorine, the biological and chemical inertness of fluorine and the FDA approved fluorine emulsions provide many opportunities for clinical hybrid ${ }^{1} \mathrm{H} /{ }^{19} \mathrm{~F}$ MRI. 


\subsection{Summary}

An overview of emerging hybrid ${ }^{1} \mathrm{H} /{ }^{19} \mathrm{~F}$ MR imaging and spectroscopy applications is provided to improve clinical diagnostics. A wide range of pre-clinical applications was presented, whereas the measurement of drug distribution and metabolism is the most foreseen application in humans (Table 4.2). The current developments in fluorinated contrast media and MR techniques can enable novel clinical diagnostic tools such as lung ventilation and angiography. The design of biocompatible and clinical approval of fluorine containing contrast media for molecular MRI can further boost the clinical applications of hybrid ${ }^{1} \mathrm{H} /{ }^{19} \mathrm{~F}$ MR imaging. Although the reported ranges of resolution, measurements times and concentration detection limits become reasonable for patients, further developments are still needed to achieve true clinical benefits. 


\section{References}

1. Mulder, W.J., G.J. Strijkers, E. Vucic, D.P. Cormode, K. Nicolay, and Z.A. Fayad, Magnetic resonance molecular imaging contrast agents and their application in atherosclerosis. Top Magn Reson Imaging, 2007. 18(5): p. 409-17.

2. Thomas, S.R., J.L. Ackerman, J.R. Goebel, M. Davis, J.G. Kereiakes, and Y.Y. Lin, Nuclear magnetic resonance imaging techniques as developed modestly within a university medical center environment: what can the small system contribute at this point? Magn Reson Imaging, 1982. 1(1): p. 11-22.

3. Clark, L.C., Jr., J.L. Ackerman, S.R. Thomas, R.W. Millard, R.E. Hoffman, R.G. Pratt, H. Ragle-Cole, R.A. Kinsey, and R. Janakiraman, Perfluorinated organic liquids and emulsions as biocompatible NMR imaging agents for ${ }^{19} \mathrm{~F}$ and dissolved oxygen. Advances in experimental medicine and biology, 1984. 180: p. 835-45.

4. Hinshaw, W.S., P.A. Bottomley, and G.N. Holland, Radiographic thin-section image of the human wrist by nuclear magnetic resonance. Nature, 1977. 270(5639): p. 722-3.

5. Noth, U., L.M. Rodrigues, S.P. Robinson, A. Jork, U. Zimmermann, B. Newell, and J.R. Griffiths, In vivo determination of tumor oxygenation during growth and in response to carbogen breathing using 15C5-loaded alginate capsules as fluorine-19 magnetic resonance imaging oxygen sensors. Int J Radiat Oncol Biol Phys, 2004. 60(3): p. 909-19.

6. Magat, J., B.F. Jordan, G.O. Cron, and B. Gallez, Noninvasive mapping of spontaneous fluctuations in tumor oxygenation using ${ }^{19} \mathrm{~F}$ MRI. Med Phys, 2010. 37(10): p. 5434-41.

7. Myerson, J., L. He, G. Lanza, D. Tollefsen, and S. Wickline, Thrombin-inhibiting perfluorocarbon nanoparticles provide a novel strategy for the treatment and magnetic resonance imaging of acute thrombosis. J Thromb Haemost, 2011. 9(7): p. 1292-300.

8. Morawski, A.M., P.M. Winter, X. Yu, R.W. Fuhrhop, M.J. Scott, F. Hockett, J.D. Robertson, P.J. Gaffney, G.M. Lanza, and S.A. Wickline, Quantitative "magnetic resonance immunohistochemistry" with ligand-targeted (19)F nanoparticles. Magn Reson Med, 2004. 52(6): p. 1255-62.

9. Srinivas, M., A. Heerschap, E.T. Ahrens, C.G. Figdor, and I.J. de Vries, ${ }^{19}$ F MRI for quantitative in vivo cell tracking. Trends Biotechnol, 2010. 28(7): p. 363-70.

10. McIntyre, D.J., F.A. Howe, C. Ladroue, F. Lofts, M. Stubbs, and J.R. Griffiths, Can localised ${ }^{19} \mathrm{~F}$ magnetic resonance spectroscopy pharmacokinetics of $5 \mathrm{FU}$ in colorectal metastases predict clinical response? Cancer Chemother Pharmacol, 2011. 68(1): p. 29-36.

11. Schlemmer, H.P., M. Becker, P. Bachert, A. Dietz, V. Rudat, B. Vanselow, P. Wollensack, I. Zuna, M.V. Knopp, H. Weidauer, M. Wannenmacher, and G. van Kaick, Alterations of intratumoral pharmacokinetics of 5-fluorouracil in head and neck carcinoma during simultaneous radiochemotherapy. Cancer Res, 1999. 59(10): p. 2363-9.

12. Ruiz-Cabello, J., B.P. Barnett, P.A. Bottomley, and J.W. Bulte, Fluorine ( $\left.{ }^{19} \mathrm{~F}\right) \mathrm{MRS}$ and MRI in biomedicine. NMR Biomed, 2010. 24(2): p. 114-29.

13. Chen, J.J., G.M. Lanza, and S.A. Wickline, Quantitative magnetic resonance fluorine imaging: today and tomorrow. Wiley Interdisciplinary Reviews-Nanomedicine and Nanobiotechnology, 2010. 2(4): p. 431-440.

14. Diaz-Lopez, R., N. Tsapis, and E. Fattal, Liquid perfluorocarbons as contrast agents for ultrasonography and (19)F-MRI. Pharm Res, 2010. 27(1): p. 1-16. 
15. Lanza, G.M., P.M. Winter, A.M. Neubauer, S.D. Caruthers, F.D. Hockett, and S.A. Wickline, H-1/F-19 magnetic resonance molecular imaging with perfluorocarbon nanoparticles. Current Topics in Developmental Biology, Volume 70, 2005. 70: p. 57-76.

16. Wolf, W., C.A. Presant, and V. Waluch, ${ }^{19} \mathrm{~F}-\mathrm{MRS}$ studies of fluorinated drugs in humans. Adv Drug Deliv Rev, 2000. 41(1): p. 55-74.

17. Stoll, G., T. Basse-Lusebrink, G. Weise, and P. Jakob, Visualization of inflammation using (19) F-magnetic resonance imaging and perfluorocarbons. Wiley interdisciplinary reviews. Nanomedicine and nanobiotechnology, 2012. 4(4): p. 438-47.

18. Temme, S., F. Bonner, J. Schrader, and U. Flogel, ${ }^{19} \mathrm{~F}$ magnetic resonance imaging of endogenous macrophages in inflammation. Wiley interdisciplinary reviews. Nanomedicine and nanobiotechnology, 2012. 4(3): p. 329-43.

19. Dardzinski, B.J. and C.H. Sotak, Rapid tissue oxygen tension mapping using ${ }^{19} \mathrm{~F}$ inversionrecovery echo-planar imaging of perfluoro-15-crown-5-ether. Magn Reson Med, 1994. 32(1): p. 88-97.

20. Giraudeau, C., J. Flament, B. Marty, F. Boumezbeur, S. Meriaux, C. Robic, M. Port, N. Tsapis, E. Fattal, E. Giacomini, F. Lethimonnier, D. Le Bihan, and J. Valette, A new paradigm for high-sensitivity ${ }^{19} \mathrm{~F}$ magnetic resonance imaging of perfluorooctylbromide. Magn Reson Med, 2010. 63(4): p. 1119-24.

21. Chekmenev, E.Y., S.K. Chow, D. Tofan, D.P. Weitekamp, B.D. Ross, and P. Bhattacharya, Fluorine-19 NMR chemical shift probes molecular binding to lipid membranes. J Phys Chem B, 2008. 112(20): p. 6285-7.

22. Bachert, P. and L. Schröder, Magnetic resonance spectroscopy.Part 1: basics. Radiologe 2003. 43(1): p. 113-1128.

23. MacKenzie, K.J.D. and M.E. Smith, Multinuclear Solid-State Nuclear Magnetic Resonance of Inorganic Materials Pergamon Materials Series,, ed. R.W. Cahn. 2004 Pergamon. 727.

24. Jerusalem, T.H.U.o. 200819 February 2008 [cited 20085 October]; Department of Organic Chemistry. The NMR Lab]. Available from: http://chem.ch.huji.ac.il/nmr/index.html.

25. Bringmann, G., K. Wolf, M. Meininger, M. Rokitta, and A. Haase, In vivo ${ }^{19} \mathrm{~F}$ NMR chemicalshift imaging of Ancistrocladus species. Protoplasma, 2001. 218(3-4): p. 134-43.

26. Srinivas, M., L.J. Cruz, F. Bonetto, A. Heerschap, C.G. Figdor, and I.J. de Vries, Customizable, multi-functional fluorocarbon nanoparticles for quantitative in vivo imaging using ${ }^{19} \mathrm{~F} \mathrm{MRI}$ and optical imaging. Biomaterials, 2010. 31(27): p. 7070-7.

27. Wilson, S.R. and P.N. Burns, Microbubble-enhanced US in body imaging: what role? Radiology, 2010. 257(1): p. 24-39.

28. Barnett, B.P., J. Ruiz-Cabello, P. Hota, R. Liddell, P. Walczak, V. Howland, V.P. Chacko, D.L. Kraitchman, A. Arepally, and J.W. Bulte, Fluorocapsules for improved function, immunoprotection, and visualization of cellular therapeutics with MR, US, and CT imaging. Radiology, 2010. 258(1): p. 182-91.

29. Cormode, D.P., W.J. Mulder, and Z.A. Fayad, Science to practice: versatile method to track transplanted encapsulated islet cells with multiple imaging modalities. Radiology, 2011. 258(1): p. 1-2.

30. Kornmann, L.M., K.D. Reesink, R.S. Reneman, and A.P. Hoeks, Critical appraisal of targeted ultrasound contrast agents for molecular imaging in large arteries. Ultrasound Med Biol, 2009. 36(2): p. 181-91. 
31. Park, B.K., N.R. Kitteringham, and P.M. O'Neill, Metabolism of fluorine-containing drugs. Annual Review of Pharmacology and Toxicology, 2001. 41: p. 443-470.

32. Muller, K., C. Faeh, and F. Diederich, Fluorine in pharmaceuticals: looking beyond intuition. Science, 2007. 317(5846): p. 1881-6.

33. Huiming, Z. Perfluoro-15-crown-5 ether-labeled dendritic cells PFPE-DCs. National Center for Biotechnology Information].

34. Schwitter, J., Extending the frontiers of cardiac magnetic resonance. Circulation, 2008. 118(2): p. 109-12.

35. Nieuwoudt, M., G.H. Engelbrecht, L. Sentle, R. Auer, D. Kahn, and S.W. van der Merwe, Non-toxicity of IV injected perfluorocarbon oxygen carrier in an animal model of liver regeneration following surgical injury. Artif Cells Blood Substit Immobil Biotechnol, 2009. 37(3): p. 117-24.

36. Krafft, M.P. and J.G. Riess, Perfluorocarbons: Life sciences and biomedical uses - Dedicated to the memory of Professor Guy Ourisson, a true RENAISSANCE man. Journal of Polymer Science Part a-Polymer Chemistry, 2007. 45(7): p. 1185-1198.

37. Riess, J.G., Perfluorocarbon-based oxygen delivery. Artificial Cells Blood Substitutes and Biotechnology, 2006. 34(6): p. 567-580.

38. Riess, J.G., Fluorocarbon-based in vivo oxygen transport and delivery systems. Vox Sang, 1991. 61(4): p. 225-39.

39. Hess, J.R., Update on alternative oxygen carriers. Vox Sanguinis, 2004. 87: p. 132-135.

40. Jahr, J.S., V. Walker, and K. Manoochehri, Blood substitutes as pharmacotherapies in clinical practice. Current opinion in anaesthesiology, 2007. 20(4): p. 325-30.

41. Ramaesh, K., S. Bhagat, S.B. Wharton, and J. Singh, Corneal epithelial toxic effects and inflammatory response to perfluorocarbon liquid. Arch Ophthalmol, 1999. 117(10): p. 1411-3.

42. Castro, C.I. and J.C. Briceno, Perfluorocarbon-based oxygen carriers: review of products and trials. Artif Organs, 2010. 34(8): p. 622-34.

43. Thyes, C. and D.R. Spahn, Current status of artificial 02 carriers. Anesthesiology clinics of North America, 2005. 23(2): p. 373-89, viii.

44. Riess, J.G. and M.P. Krafft, eds. Fluorocarbon emulsions as in vivo oxygen delivery systems: background and chemistry. Blood Substitutes, ed. W.R. M. 2006, Academic Press: London. 259-75.

45. Pfannkuch, F. and N. Schnoy, Perfluorochemical-emulsion (fluorocarbon-43) as blood gas carrier--fate of the substance after intravenous injection in rat (author's transl). Anaesthesist, 1979. 28(11): p. 511-6.

46. Code, R.F., J.E. Harrison, and K.G. McNeill, In vivo measurement of accumulated bone fluorides by nuclear magnetic resonance. Journal of bone and mineral research : the official journal of the American Society for Bone and Mineral Research, 1990. 5 Suppl 1: p. S91-4.

47. Klomp, D., H. van Laarhoven, T. Scheenen, Y. Kamm, and A. Heerschap, Quantitative ${ }^{19} \mathrm{~F}$ MR spectroscopy at $3 \mathrm{~T}$ to detect heterogeneous capecitabine metabolism in human liver. NMR Biomed, 2007. 20(5): p. 485-92. 
48. Bolo, N.R., Y. Hode, and J.P. Macher, Long-term sequestration of fluorinated compounds in tissues after fluvoxamine or fluoxetine treatment: a fluorine magnetic resonance spectroscopy study in vivo. MAGMA, 2004. 16(6): p. 268-76.

49. Bolo, N.R., Y. Hode, J.F. Nedelec, E. Laine, G. Wagner, and J.P. Macher, Brain pharmacokinetics and tissue distribution in vivo of fluvoxamine and fluoxetine by fluorine magnetic resonance spectroscopy. Neuropsychopharmacology : official publication of the American College of Neuropsychopharmacology, 2000. 23(4): p. 428-38.

50. Schneider, E., N.R. Bolo, B. Frederick, S. Wilkinson, F. Hirashima, L. Nassar, I.K. Lyoo, P. Koch, S. Jones, J. Hwang, Y. Sung, R.A. Villafuerte, G. Maier, R. Hsu, R. Hashoian, and P.F. Renshaw, Magnetic resonance spectroscopy for measuring the biodistribution and in situ in vivo pharmacokinetics of fluorinated compounds: validation using an investigation of liver and heart disposition of tecastemizole. Journal of clinical pharmacy and therapeutics, 2006. 31(3): p. 261-73.

51. Huang, Z., R.S. Sengar, A. Nigam, M.C. Abadjian, D.M. Potter, D.B. Grotjahn, and E.C. Wiener, A fluorinated dendrimer-based nanotechnology platform: new contrast agents for high field imaging. Invest Radiol, 2010. 45(10): p. 641-54.

52. van Laarhoven, H.W., D.W. Klomp, Y.J. Kamm, C.J. Punt, and A. Heerschap, In vivo monitoring of capecitabine metabolism in human liver by 19 fluorine magnetic resonance spectroscopy at 1.5 and 3 Tesla field strength. Cancer Res, 2003. 63(22): p. 7609-12.

53. Reid, D.G. and P.S. Murphy, Fluorine magnetic resonance in vivo: a powerful tool in the study of drug distribution and metabolism. Drug Discov Today, 2008. 13(11-12): p. 473-80.

54. Sassa, T., T. Suhara, H. Ikehira, T. Obata, F. Girard, S. Tanada, and Y. Okubo, ${ }^{19}$ F-magnetic resonance spectroscopy and chemical shift imaging for schizophrenic patients using haloperidol decanoate. Psychiatry Clin Neurosci, 2002. 56(6): p. 637-42.

55. Klomp, D.W., H.W. Van Laarhoven, A.P. Kentgens, and A. Heerschap, Optimization of localized ${ }^{19} \mathrm{~F}$ magnetic resonance spectroscopy for the detection of fluorinated drugs in the human liver. Magn Reson Med, 2003. 50(2): p. 303-8.

56. Bilecen, D., A.C. Schulte, A. Kaspar, E. Kustermann, J. Seelig, D. Elverfeldt, and K. Scheffler, Detection of the non-steroidal anti-inflammatory drug niflumic acid in humans: a combined ${ }^{19} \mathrm{~F}-\mathrm{MRS}$ in vivo and in vitro study. NMR in biomedicine, 2003. 16(3): p. 144-51.

57. Payne, G.S., D.J. Collins, P. Loynds, G. Mould, P.S. Murphy, A.S. Dzik-Jurasz, P. Kessar, N. Haque, M. Yamaguchi, S. Atarashi, and M.O. Leach, Quantitative assessment of the hepatic pharmacokinetics of the antimicrobial sitafloxacin in humans using in vivoF magnetic resonance spectroscopy. British journal of clinical pharmacology, 2005. 59(2): p. 244-8.

58. Strauss, W.L., A.S. Unis, C. Cowan, G. Dawson, and S.R. Dager, Fluorine magnetic resonance spectroscopy measurement of brain fluvoxamine and fluoxetine in pediatric patients treated for pervasive developmental disorders. The American journal of psychiatry, 2002. 159(5): p. 755-60.

59. Henry, M.E., C.M. Moore, M.J. Kaufman, D. Michelson, M.E. Schmidt, E. Stoddard, A.J. Vuckevic, P.J. Berreira, B.M. Cohen, and P.F. Renshaw, Brain kinetics of paroxetine and fluoxetine on the third day of placebo substitution: a fluorine MRS study. The American journal of psychiatry, 2000. 157(9): p. 1506-8.

60. Strauss, W.L. and S.R. Dager, Magnetization transfer of fluoxetine in the human brain using fluorine magnetic resonance spectroscopy. Biological psychiatry, 2001. 49(9): p. 798-802. 
61. Miner, C.M., J.R. Davidson, N.L. Potts, L.A. Tupler, H.C. Charles, and K.R. Krishnan, Brain fluoxetine measurements using fluorine magnetic resonance spectroscopy in patients with social phobia. Biological psychiatry, 1995. 38(10): p. 696-8.

62. Komoroski, R.A., J.E. Newton, C. Karson, D. Cardwell, and J. Sprigg, Detection of psychoactive drugs in vivo in humans using ${ }^{19} \mathrm{~F}$ NMR spectroscopy. Biological psychiatry, 1991. 29(7): p. 711-4.

63. Durst, P., N. Schuff, M.A. Crocq, M.C. Mokrani, and J.P. Macher, Noninvasive in vivo detection of a fluorinated neuroleptic in the human brain by ${ }^{19} \mathrm{~F}$ nuclear magnetic resonance spectroscopy. Psychiatry research, 1990. 35(2): p. 107-14.

64. Procissi, D., F. Claus, P. Burgman, J. Koziorowski, J.D. Chapman, S.B. Thakur, C. Matei, C.C. Ling, and J.A. Koutcher, In vivo ${ }^{19} \mathrm{~F}$ magnetic resonance spectroscopy and chemical shift imaging of tri-fluoro-nitroimidazole as a potential hypoxia reporter in solid tumors. Clin Cancer Res, 2007. 13(12): p. 3738-47.

65. Li, C.W., W.G. Negendank, K.A. Padavic-Shaller, P.J. O’Dwyer, J. Murphy-Boesch, and T.R. Brown, Quantitation of 5-fluorouracil catabolism in human liver in vivo by three-dimensional localized ${ }^{19} \mathrm{~F}$ magnetic resonance spectroscopy. Clin Cancer Res, 1996. 2(2): p. 339-45.

66. Hideto, K., D. Yoshihiro, and K. Yoko, Application of ${ }^{19} \mathrm{~F}$ chemical shift imaging in studies of mice with orally administered 5-fluorouracil. Magnetic Resonance in Medicine, 2001. 46(5): p. 864-869.

67. Kadayakkara, D.K., J.M. Janjic, L.K. Pusateri, W.B. Young, and E.T. Ahrens, In vivo observation of intracellular oximetry in perfluorocarbon-labeled glioma cells and chemotherapeutic response in the CNS using fluorine-19 MRI. Magn Reson Med, 2010. 64(5): p. 1252-9.

68. Waters, E.A., J. Chen, X. Yang, H. Zhang, R. Neumann, A. Santeford, J. Arbeit, G.M. Lanza, and S.A. Wickline, Detection of targeted perfluorocarbon nanoparticle binding using ${ }^{19} \mathrm{~F}$ diffusion weighted MR spectroscopy. Magn Reson Med, 2008. 60(5): p. 1232-6.

69. Winter, P.M., A.M. Neubauer, S.D. Caruthers, T.D. Harris, J.D. Robertson, T.A. Williams, A.H. Schmieder, G. Hu, J.S. Allen, E.K. Lacy, H. Zhang, S.A. Wickline, and G.M. Lanza, Endothelial alpha(v)beta3 integrin-targeted fumagillin nanoparticles inhibit angiogenesis in atherosclerosis. Arterioscler Thromb Vasc Biol, 2006. 26(9): p. 2103-9.

70. Flogel, U., Z. Ding, H. Hardung, S. Jander, G. Reichmann, C. Jacoby, R. Schubert, and J. Schrader, In vivo monitoring of inflammation after cardiac and cerebral ischemia by fluorine magnetic resonance imaging. Circulation, 2008. 118(2): p. 140-8.

71. Neubauer, A.M., S.D. Caruthers, F.D. Hockett, T. Cyrus, J.D. Robertson, J.S. Allen, T.D. Williams, R.W. Fuhrhop, G.M. Lanza, and S.A. Wickline, Fluorine cardiovascular magnetic resonance angiography in vivo at $1.5 \mathrm{~T}$ with perfluorocarbon nanoparticle contrast agents. J Cardiovasc Magn Reson, 2007. 9(3): p. 565-73.

72. Waters, E.A., J. Chen, J.S. Allen, H. Zhang, G.M. Lanza, and S.A. Wickline, Detection and quantification of angiogenesis in experimental valve disease with integrin-targeted nanoparticles and 19-fluorine MRI/MRS. J Cardiovasc Magn Reson, 2008. 10: p. 43.

73. de Lange, E.E., T.A. Altes, J.T. Patrie, J.J. Battiston, A.P. Juersivich, J.P. Mugler, 3rd, and T.A. Platts-Mills, Changes in regional airflow obstruction over time in the lungs of patients with asthma: evaluation with 3He MR imaging. Radiology, 2009. 250(2): p. 567-75.

74. Loring, S.H., J.P. Butler, and S. Patz, Science to practice: how do we interpret the transfer of hyperpolarized 129Xe from blood into alveolar gas? Radiology, 2009. 252(2): p. 319-21. 
75. Mosbah, K., J. Ruiz-Cabello, Y. Berthezene, and Y. Cremillieux, Aerosols and gaseous contrast agents for magnetic resonance imaging of the lung. Contrast media \& molecular imaging, 2008. 3(5): p. 173-90.

76. Kuethe, D.O., A. Caprihan, H.M. Gach, I.J. Lowe, and E. Fukushima, Imaging obstructed ventilation with NMR using inert fluorinated gases. Journal of Applied Physiology, 2000. 88(6): p. 2279-2286.

77. Wolf, U., A. Scholz, C.P. Heussel, K. Markstaller, and W.G. Schreiber, Subsecond fluorine-19 MRI of the lung. Magn Reson Med, 2006. 55(4): p. 948-51.

78. Adolphi, N.L. and D.O. Kuethe, Quantitative mapping of ventilation-perfusion ratios in lungs by ${ }^{19} \mathrm{~F}$ MR imaging of $T_{1}$ of inert fluorinated gases. Magn Reson Med, 2008. 59(4): p. 73946.

79. Scholz, A.W., B. Eberle, C.P. Heussel, M. David, M.D. Schmittner, M. Quintel, L.M. Schreiber, and N. Weiler, Ventilation-perfusion ratio in perflubron during partial liquid ventilation. Anesth Analg, 2010. 110(6): p. 1661-8.

80. Wolf, U., A. Scholz, M. Terekhov, K. Muennemann, K. Kreitner, C. Werner, C. Dueber, and W.G. Schreiber. Fluorine-19 MRI of the lung: first human experiment. in ISMRM 2008. 2008.

81. Miyazaki, M. and V.S. Lee, Nonenhanced MR angiography. Radiology, 2008. 248(1): p. 2043.

82. van Heeswijk, R.B., Y. Pilloud, U. Flogel, J. Schwitter, and M. Stuber, Fluorine-19 magnetic resonance angiography of the mouse. PLoS One, 2012. 7(7): p. e42236.

83. Riess, J.G., Overview of progress in the fluorocarbon approach to in vivo oxygen delivery. Biomater Artif Cells Immobilization Biotechnol, 1992. 20(2-4): p. 183-202.

84. Kocian, R. and D.R. Spahn, Haemoglobin, oxygen carriers and perioperative organ perfusion. Best Pract Res Clin Anaesthesiol, 2008. 22(1): p. 63-80.

85. Spahn, D.R., B.J. Leone, J.G. Reves, and T. Pasch, Cardiovascular and coronary physiology of acute isovolemic hemodilution: a review of nonoxygen-carrying and oxygen-carrying solutions. Anesth Analg, 1994. 78(5): p. 1000-21.

86. Spahn, D.R., Blood substitutes. Artificial oxygen carriers: perfluorocarbon emulsions. Crit Care, 1999. 3(5): p. R93-7.

87. Kataoka, K., G.S. Kwon, M. Yokoyama, T. Okano, and Y. Sakurai, Block-Copolymer Micelles as Vehicles for Drug Delivery. Journal of Controlled Release, 1993. 24(1-3): p. 119-132.

88. Keipert, P.E., Use of Oxygent, a perfluorochemical-based oxygen carrier, as an alternative to intraoperative blood transfusion. Artificial cells, blood substitutes, and immobilization biotechnology, 1995. 23(3): p. 381-94.

89. Massoud, T.F. and S.S. Gambhir, Molecular imaging in living subjects: seeing fundamental biological processes in a new light. Genes Dev, 2003. 17(5): p. 545-80.

90. Margolis, D.J., J.M. Hoffman, R.J. Herfkens, R.B. Jeffrey, A. Quon, and S.S. Gambhir, Molecular imaging techniques in body imaging. Radiology, 2007. 245(2): p. 333-56.

91. Rudin, M., M. Rausch, and M. Stoeckli, Molecular imaging in drug discovery and development: potential and limitations of nonnuclear methods. Mol Imaging Biol, 2005. 7(1): p. 5-13.

92. Jaffer, F.A. and R. Weissleder, Molecular imaging in the clinical arena. JAMA, 2005. 293(7): p. 855-62. 
93. Mulder, W.J., G.J. Strijkers, G.A. van Tilborg, A.W. Griffioen, and K. Nicolay, Lipid-based nanoparticles for contrast-enhanced MRI and molecular imaging. NMR Biomed, 2006. 19(1): p. 142-64.

94. Sipkins, D.A., D.A. Cheresh, M.R. Kazemi, L.M. Nevin, M.D. Bednarski, and K.C. Li, Detection of tumor angiogenesis in vivo by alphaVbeta3-targeted magnetic resonance imaging. Nat Med, 1998. 4(5): p. 623-6.

95. Weissleder, R., Molecular imaging in cancer. Science, 2006. 312(5777): p. 1168-71.

96. Oostendorp, M., M.J. Post, and W.H. Backes, Vessel growth and function: depiction with contrast-enhanced MR imaging. Radiology, 2009. 251(2): p. 317-35.

97. Winter, P.M., S.D. Caruthers, X. Yu, S.K. Song, J. Chen, B. Miller, J.W. Bulte, J.D. Robertson, P.J. Gaffney, S.A. Wickline, and G.M. Lanza, Improved molecular imaging contrast agent for detection of human thrombus. Magn Reson Med, 2003. 50(2): p. 411-6.

98. Flacke, S., S. Fischer, M.J. Scott, R.J. Fuhrhop, J.S. Allen, M. McLean, P. Winter, G.A. Sicard, P.J. Gaffney, S.A. Wickline, and G.M. Lanza, Novel MRI contrast agent for molecular imaging of fibrin: implications for detecting vulnerable plaques. Circulation, 2001. 104(11): p. $1280-5$.

99. Cyrus, T., G.M. Lanza, and S.A. Wickline, Molecular imaging by cardiovascular MR. J Cardiovasc Magn Reson, 2007. 9(6): p. 827-43.

100. Caruthers, S.D., In Vitro Demonstration Using ${ }^{19} \mathrm{~F}$ MR to Augment Molecular Imaging With Paramagnetic Perfluorocarbon Nanoparticles at 1.5 Tesla. Investigative Radiology, 2006. 41(3): p. 305-312.

101. Partlow, K.C., J. Chen, J.A. Brant, A.M. Neubauer, T.E. Meyerrose, M.H. Creer, J.A. Nolta, S.D. Caruthers, G.M. Lanza, and S.A. Wickline, ${ }^{19} \mathrm{~F}$ magnetic resonance imaging for stem/ progenitor cell tracking with multiple unique perfluorocarbon nanobeacons. FASEB J, 2007. 21(8): p. 1647-54.

102. Keupp, J., J. Rahmer, I. Grasslin, P.C. Mazurkewitz, T. Schaeffter, G.M. Lanza, S.A. Wickline, and S.D. Caruthers, Simultaneous dual-nuclei imaging for motion corrected detection and quantification of ${ }^{19} \mathrm{~F}$ imaging agents. Magn Reson Med, 2011. 66(4): p. 1116-22.

103. Wolters, M., M. Oostendorp, B.F. Coolen, M.J. Post, J.M. Janssen, G.J. Strijkers, M.E. Kooi, K. Nicolay, and W.H. Backes, Efficacy of positive contrast imaging techniques for molecular MRI of tumor angiogenesis. Contrast Media Mol Imaging, 2012. 7(2): p. 130-9.

104. Skobe, M., P. Rockwell, N. Goldstein, S. Vosseler, and N.E. Fusenig, Halting angiogenesis suppresses carcinoma cell invasion. Nat Med, 1997. 3(11): p. 1222-7.

105. Orlic, D., J. Kajstura, S. Chimenti, I. Jakoniuk, S.M. Anderson, B. Li, J. Pickel, R. McKay, B. Nadal-Ginard, D.M. Bodine, A. Leri, and P. Anversa, Bone marrow cells regenerate infarcted myocardium. Nature, 2001. 410(6829): p. 701-5.

106. Genovese, J., M. Cortes-Morichetti, E. Chachques, G. Frati, A. Patel, and J.C. Chachques, Cell based approaches for myocardial regeneration and artificial myocardium. Current stem cell research \& therapy, 2007. 2(2): p. 121-7.

107. Pera, M.F. and P.P. Tam, Extrinsic regulation of pluripotent stem cells. Nature, 2010. 465(7299): p. 713-20.

108. Martino, G., R.J. Franklin, A. Baron Van Evercooren, and D.A. Kerr, Stem cell transplantation in multiple sclerosis: current status and future prospects. Nature reviews. Neurology, 2010. 6(5): p. 247-55. 
109. Kerkar, S.P. and N.P. Restifo, Cellular constituents of immune escape within the tumor microenvironment. Cancer research, 2012. 72(13): p. 3125-30.

110. Srinivas, M., E.H. Aarntzen, J.W. Bulte, W.J. Oyen, A. Heerschap, I.J. de Vries, and C.G. Figdor, Imaging of cellular therapies. Advanced drug delivery reviews, 2010. 62(11): p. 1080-93.

111. Ahrens, E.T., R. Flores, H. Xu, and P.A. Morel, In vivo imaging platform for tracking immunotherapeutic cells. Nat Biotechnol, 2005. 23(8): p. 983-7.

112. Ebner, B., P. Behm, C. Jacoby, S. Burghoff, B.A. French, J. Schrader, and U. Flogel, Early assessment of pulmonary inflammation by ${ }^{19} \mathrm{~F}$ MRI in vivo. Circ Cardiovasc Imaging, 2010. 3(2): p. 202-10.

113. Flogel, U., S. Su, I. Kreideweiss, Z. Ding, L. Galbarz, J. Fu, C. Jacoby, O. Witzke, and J. Schrader, Noninvasive detection of graft rejection by in vivo (19) F MRI in the early stage. Am J Transplant, 2011. 11(2): p. 235-44.

114. Weise, G., T.C. Basse-Lusebrink, C. Kleinschnitz, T. Kampf, P.M. Jakob, and G. Stoll, In vivo imaging of stepwise vessel occlusion in cerebral photothrombosis of mice by ${ }^{19} \mathrm{~F}$ MRI. PloS one, 2011. 6(12): p. e28143.

115. Trott, C.M. and G. El Fakhri, Sequential and simultaneous dual-isotope brain SPECT: comparison with PET for estimation and discrimination tasks in early Parkinson disease. Med Phys, 2008. 35(7): p. 3343-53.

116. Hsieh, P.C., I.H. Lee, T.L. Yeh, K.C. Chen, H.C. Huang, P.S. Chen, Y.K. Yang, W.J. Yao, R.B. $\mathrm{Lu}$, and N.T. Chiu, Distribution volume ratio of serotonin and dopamine transporters in euthymic patients with a history of major depression - a dual-isotope SPECT study. Psychiatry Res, 2010. 184(3): p. 157-61.

117. Hachamovitch, R., X. Kang, A.M. Amanullah, A. Abidov, S.W. Hayes, J.D. Friedman, I. Cohen, L.E. Thomson, G. Germano, and D.S. Berman, Prognostic implications of myocardial perfusion single-photon emission computed tomography in the elderly. Circulation, 2009. 120(22): p. 2197-206.

118. Miserus, R.J., M.V. Herias, L. Prinzen, M.B. Lobbes, R.J. Van Suylen, A. Dirksen, T.M. Hackeng, J.W. Heemskerk, J.M. van Engelshoven, M.J. Daemen, M.A. van Zandvoort, S. Heeneman, and M.E. Kooi, Molecular MRI of early thrombus formation using a bimodal alpha2-antiplasmin-based contrast agent. JACC Cardiovasc Imaging, 2009. 2(8): p. 98796.

119. Mistretta, C.A., Undersampled radial MR acquisition and highly constrained back projection (HYPR) reconstruction: potential medical imaging applications in the post-Nyquist era. Journal of magnetic resonance imaging : JMRI, 2009. 29(3): p. 501-16.

120.Kampf, T., A. Fischer, T.C. Basse-Lusebrink, G. Ladewig, F. Breuer, G. Stoll, P.M. Jakob, and W.R. Bauer, Application of compressed sensing to in vivo 3D (1)(9)F CSI. Journal of magnetic resonance, 2010. 207(2): p. 262-73.

121. Keupp, J., A.H. Schmieder, T.A. Williams, J.S. Allen, S.A. Wickline, G.M. Lanza, and S.D. Caruthers. Ultra-short Echo Time ${ }^{19} \mathrm{~F} /{ }^{1} \mathrm{H}$ Imaging of Gadolinium-free Perfluoro-carbon Nanoparticles: A Robust Method for In Vivo Angiogenesis Imaging. in International Society for Magnetic Resonance in Medicine (ISMRM). 2011. Montréal. 
Chapter 4

122.Du, J., G. Hamilton, A. Takahashi, M. Bydder, and C.B. Chung, Ultrashort echo time spectroscopic imaging (UTESI) of cortical bone. Magnetic resonance in medicine : official journal of the Society of Magnetic Resonance in Medicine / Society of Magnetic Resonance in Medicine, 2007. 58(5): p. 1001-9 


\section{Molecular MRI with a fibrin specific fluorine-loaded polymer nanoparticle}

M. Wolters, M.J. van Steenbergen, W.E. Hennink, P. van de Vijver, T.M. Hackeng, M.J. Post, W.H. Backes, and M.E. Kooi
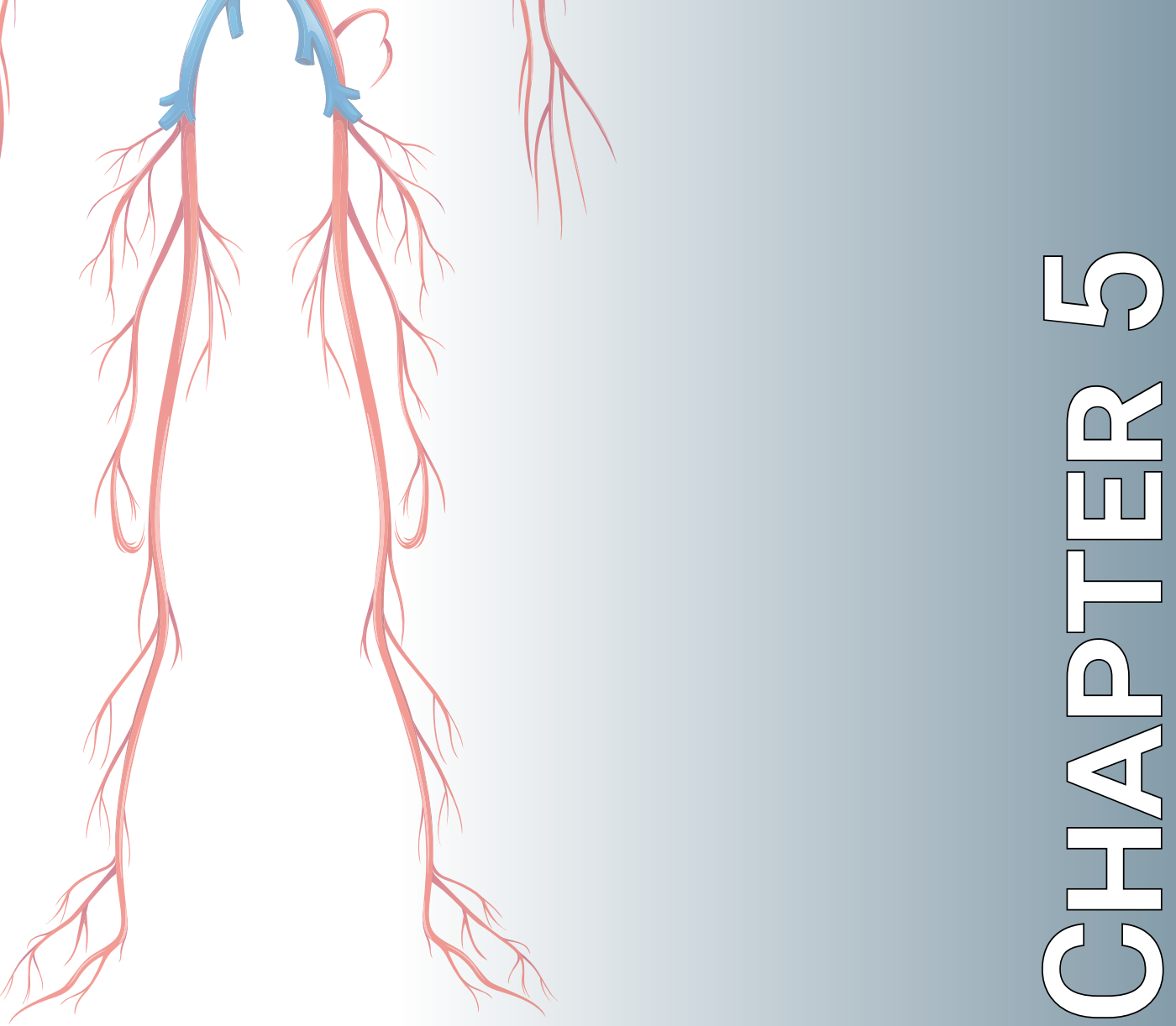
Purpose Fluorine based molecular MRI might provide a solution in the diagnosis of acute thrombi by using a contrast agent (CA) targeted to the acute thrombus. By directly detecting the fluorine signal from the CA that is bound to its molecular target, the MRI contrast is not hindered by the background (blood) signal. We investigated the feasibility of fibrin-specific A14 peptide conjugated to fluorine loaded nanoparticles (NP) for molecular MRI of thrombi.

Methods Perfluoro-15-crown-5-ether was loaded in a polymer nanoparticle, either with ( $\left.{ }^{19} \mathrm{~F}-\mathrm{NP}-\mathrm{A} 14 \mathrm{CA}\right)$ or without $\left({ }^{19} \mathrm{~F}-\mathrm{NP} \mathrm{CA}\right)$ surface bound A14 peptide as targeting ligand. Fresh plasma clots were in vitro labeled with ${ }^{19} \mathrm{~F}-\mathrm{NP}-\mathrm{A} 14$ or ${ }^{19} \mathrm{~F}-\mathrm{NP}$ CA suspension, embedded in agarose gel and imaged with MRI. MRI comprised proton and fluorine imaging, for structural imaging and CA mapping, respectively. For the in vivo experiments thrombi were induced in the carotid artery in mice. CA formulations were administered for in vivo MRI. Hereafter, the carotid arteries were excised and subsequently examined ex vivo with MRI.

Results In vivo MRI measurements revealed CA accumulation in the liver, but no ${ }^{19} \mathrm{~F}$ contrast in the thrombus within an acquisition time of 17 minutes. The in vitro and ex vivo measurements yielded a significant larger fraction of enhanced voxels with ${ }^{19} \mathrm{~F} \mathrm{MRI}(\mathrm{P}<0.05)$ in the thrombi labeled with the ${ }^{19} \mathrm{~F}-\mathrm{NP}-\mathrm{A} 14 \mathrm{CA}$ compared to the ${ }^{19} \mathrm{~F}-\mathrm{NP}$ CA.

Conclusion The novel fibrin-specific A14 fluorine loaded nanoparticle formulation showed specific affinity for fresh thrombi as demonstrated by ex vivo and in vitro fluorine MRI. In vivo imaging of this nanoparticle requires more research on more sensitive and dedicated fluorine MRI techniques. 


\subsection{Introduction}

Thromboembolism causes diseases such as deep vein thrombosis and pulmonary embolism. In addition, thromboembolism from a ruptured atherosclerotic plaque is an important underlying cause of stroke and myocardial infarction. It is therefore a major cause of morbidity and mortality. The early diagnosis of acute thromboembolism is hampered by suboptimal imaging techniques. Currently, different imaging modalities such as ultrasound, computed-tomography (CT) and Magnetic Resonance Imaging (MRI) are used for thrombus detection depending on the vascular territory. Ultrasound and MRI are used to diagnose deep vein thrombosis (DVT), whereas CT is the gold standard for the detection of pulmonary embolisms. Despite the value of these techniques, the cause of ischemic strokes is in $30-40 \%$ of the cases undefined or "cryptogenic", which means that the source of the thromboembolism is never identified ${ }^{[1]}$. The current imaging modalities are based on indirect detection of thrombi by visualizing an obstruction of the lumen and therefore cannot distinguish stable atherosclerotic plaque from plaque with associated thrombus.

Thrombi become more resistant to fibrinolytic therapy when they get older [2], and fibrinolytic agents can induce severe complications such as bleedings $[3,4]$. In clinical research settings at our institution, it has already been shown that molecular MRI can be used to visualize thrombi ${ }^{[5-7]}$ and distinguish fresh from older thrombi ${ }^{[8]}$. This can improve the selection of patients that will benefit from fibrinolytic therapy. In an ideal in vivo characterization, a thrombus should possess two distinct molecular addresses that can be targeted by contrast agents, one, for instance fibrin, indicating its presence and a second one that is associated with ageing of the thrombus. We have shown that the level of fibrin cross-linking with an alpha-2-antiplasmin based ligand, such as the A14 peptide, fits the latter description.

The current proton-based $\left({ }^{1} \mathrm{H}\right)$ CAs such as gadolinium and iron-oxide CAs are sub optimal for the imaging of multiple biomarkers due to their indirect detection method. Fluorine based CAs for molecular MRI are promising candidates for the imaging of multiple biomarkers for 3 reasons: 1) The wide chemical shift (> 200 ppm) provides the opportunity to detect and distinguish multiple fluorine CAs based on their spectral identity ${ }^{[9]}$; 2) The MR sensitivity of fluorine is close to that of protons $(83 \%)$, which makes the nucleus well detectable; 3 ) The lack of fluorine in the human body results in the absence of background (anatomy) signal, improving reliability of quantification.

So far, a few experimental studies have been published in which fluorine loaded nanoparticles were used for molecular MRI [9-14]. These liposomes nanoparticles were actively targeted by ligating peptides or antibodies to its surface. With these nanoparticles also passive targeting was performed for cell labeling or the visualization of inflammatory lesions, in which macrophages tend to take up nanoparticles ${ }^{[13,15]}$. Biodegradable polymeric nanoparticles have emerged as drug nano-carriers. These nanoparticles have the advantage of a small size, a large variety of preparation methods, high stability and versatile release profiles ${ }^{[16,17]}$. Similar to liposomes, the 
polymer surface can be pegylated and ligands, e.g. peptides, proteins or antibodies can be covalently or non-covalently coupled e.g. by streptavidin-biotin coupling [18-21].

In the present study, we investigate the feasibility of a novel A14 conjugated fluorine loaded polymer nanoparticle (NP) for fluorine MRI (Figure 5.1). Thrombi were labeled with a specific contrast agent (CA), which contains the $\mathrm{A} 14$ peptide as ligand or with a non-specific CA where the A14 peptide is absent. The structure (and localization) of the thrombi was imaged with proton MRI and the labeling of the thrombi for both CAs was assessed with fluorine MRI. The labeling with the CA was assessed in (i) in vitro induced fresh thrombi, (ii) in vivo induced thrombi in the carotid arteries of mice, and (iii) in the excised carotid arteries with thrombi from the same mice (ex vivo).
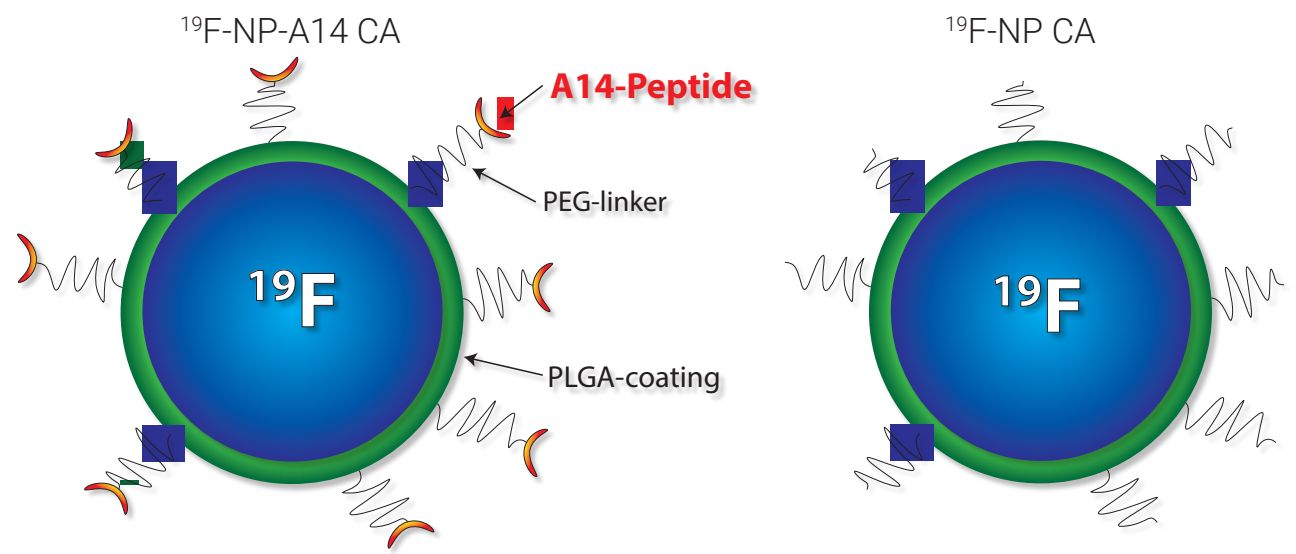

Figure 5.1 - A schematic representation of the fluorine loaded nanoparticles. This nanoparticle contains a fluorine core, a PLGA (poly(lactic-co-glycolic acid)) shell and is pegylated (PEG). The fresh thrombus targeted ${ }^{19} \mathrm{~F}-\mathrm{NP}-\mathrm{A} 14$ contrast agent (CA) contains the A14-peptide on the end terminus of the PEG-linker, whereas the ${ }^{19} \mathrm{~F}-\mathrm{NP}$ CA has no peptide on the linker. 


\subsection{Methods}

\section{Materials and synthesis of PLGA-PEG and PLGA-PEG-A14}

ForPLGA-PEG (poly(lactic-co-glycolicacid))-(poly(ethyleneglycol))randomcopolymers of glycolide and D,L-lactide were synthesized by ring opening polymerization in the melt using monomethoxypoly(ethylene glycol)2000 (MePEG2000) as initiator and stannous octoate as catalyst, respectively, essentially as described previously [22]. Briefly, to obtain LA/GA ratio 50:50 with a MW of 12000, a mixture of D,L-lactide (5.6g) and Glycolide (4.6g) was loaded into a Schlenk tube followed by the addition of stannous octoate $(200 \mathrm{mg})$ and $2 \mathrm{~g}$ MePEG2000. After removal of moisture by applying vacuum, the Schlenk tube was closed and subsequently transferred into an oil bath of $140{ }^{\circ} \mathrm{C}$. The melt polymerization proceeded overnight and after cooling to room temperature, the crude product was dissolved in chloroform, precipitated into an excess of methanol and vacuum dried overnight. The A14 peptide was synthesized as described in ${ }^{[8]}$. The peptide was covalently linked to the distal end of a Poly(lactideco-glycolide)-b-poly(ethylene glycol)-maleimide (PLGA-PEG-mal) chain obtained from PolySciTech. The peptide was added in a 10\% excess to the nanoparticles, dissolved in saline $(\mathrm{pH}=7.4)$ and was coupled by thiol/maleimide coupling chemistry at $37^{\circ} \mathrm{C}$ for 45 minutes. PLGA (Poly(lactic-co-glycolic acid)) was obtained from Sigma (MW 700017000, LA/GA ratio 50:50). Perfluoro-15-crown-5-ether was obtained from ExFluor.

\section{CA preparation}

The control CA was prepared by dissolving PLGA-PEG (3 mg) and PLGA (30 mg) in a mixture of dichloromethane $(1 \mathrm{ml})$ and methanol $(0.33 \mathrm{ml})$. Perfluoro-15-crown-5-ether (CE) $(100 \mu \mathrm{l})$ was emulsified in this polymer solution. Subsequently, an aqueous $0.5 \%$ polyvinyl alcohol buffer solution ( $5 \mathrm{ml}$ ) was added and the suspension was sonicated for $60 \mathrm{~s}$ with a tip-sonicator (MSE Mark II). Afterwards, $33 \mathrm{ml}$ of $0.5 \%$ polyvinyl alcohol buffer was added and stirred overnight to evaporate the solvents. The nanoparticles were centrifuged at $100 \mathrm{~g}$ to obtain NP pellets. Before use, the nanoparticles were dispersed in saline to a $30 \%(\mathrm{~V} / \mathrm{V})$ concentration. This suspension is further referred as the ${ }^{19} \mathrm{~F}-\mathrm{NP}$ CA. For the specific CA, the A14 peptide ${ }^{[8]}$ was covalently linked to the distal end of the PEG chain by thiol/maleimide coupling chemistry. In the preparation steps as described above, PLGA-PEG-A14 was used instead of PLGA-PEG. This suspension is further referred as the ${ }^{19} \mathrm{~F}-\mathrm{NP}-\mathrm{A} 14 \mathrm{CA}$.

\section{CA characterization}

Particle sizes were measured with Dynamic Light Scattering (DLS). For the MRI characterization, the CA was diluted in saline or human venous blood in a $10 \%$ $(\mathrm{v} / \mathrm{v})$ concentration. A $10 \%(\mathrm{~V} / \mathrm{V})$ dilution was chosen as the expected in vivo blood concentration directly after administration. The $T_{1}$ and $T_{2}$ relaxation times were measured for both dilutions on a 7T small animal system (Biospec 70/30, Bruker) with a ${ }^{1} \mathrm{H} /{ }^{19} \mathrm{~F}$ linear $35 \mathrm{~mm}$ volume coil at room temperature. The CA was measured using a series of fast spin echo inversion recovery (IR-RARE) acquisitions (echo time (TE) $8.1 \mathrm{~ms}$; repetition time (TR) $10000 \mathrm{~ms}$; inversion time (TI) 150, 250, 500, 750, $1000,1500,2000,2500,3000$ and 3500 ms; number of signal averages (NSA) 8) to determine the $T_{1}$ relaxation time. Spin echo (SE) acquisitions (TE 11, 22, 33, 44, 55, 66, 
77, 88, 99, 110, 121 and 132 ms; TR 10000 ms; NSA 8) were used to determine the $T_{2}$ relaxation time. Before image acquisition, first and second order global shimming was performed.

\section{In vitro thrombus experiment}

Model. To test the feasibility of CA labeling under ideal conditions (i.e. without physiological blood flow) an in vitro model was used. Human blood was obtained by venous puncture from a healthy volunteer and collected in vacutainer tubes containing trisodium citrate (BD Vacutainer Systems, Preanalytical solutions, Plymouth, UK). Three clots were prepared of $200 \mu \mathrm{L}$ for each CA, which were activated with an $\mathrm{CaCl} 2$ solution $(1 \mathrm{mmol} / \mathrm{mL}, 6 \mu \mathrm{L})$ and tissue factor $(5 \mu \mathrm{L}$ Innovin) and incubated for 45 minutes at $37^{\circ} \mathrm{C}$. The clots were then transferred into $100 \mu \mathrm{L}$ saline containing $5 \%$ $(\mathrm{v} / \mathrm{v}) \mathrm{CA}$ nanoparticles and incubated for 45 minutes at $37^{\circ} \mathrm{C}$. The clots were washed three times in saline and subsequently embedded in $2 \%$ agarose gel.

Imaging. MRI was performed with the same ${ }^{1} \mathrm{H} /{ }^{19} \mathrm{~F}$ linear volume coil. After a scout scan, ${ }^{1} \mathrm{H}$ 2D Fast Spin Echo (FSE) images were acquired (TE 9 ms; TR 1500 ms; acceleration factor 4; NSA 1; acquired and reconstructed pixel size $0.12 \times 0.12 \mathrm{~mm}^{2}$, number of slices 30; slice thickness $1.00 \mathrm{~mm}$ and field of view $30 \times 30 \times 30 \mathrm{~mm}^{3}$ ). For the ${ }^{19} \mathrm{~F}$ images, the coil was tuned to $282 \mathrm{MHz}$ and $2 \mathrm{D}$ fast spin echo images were acquired (TE 9 ms; TR 1500 ms; acceleration factor 4; NSA 16; acquired and reconstructed pixel size $0.47 \times 0.47 \mathrm{~mm}$, number of slices 15; slice thickness $2.00 \mathrm{~mm}$, and field of view $30 \times 30 \times 30 \mathrm{~mm}^{3}$ ).

\section{In vivo thrombus experiment}

Model. To test the CA labeling under physiological conditions a mouse animal model was used, which was approved by the institutional animal welfare committee. Twelveweek-old female Swiss mice (Charles River Laboratories, L'Arbresle cedex, France) were anesthetized using $1.5-2.0 \%$ isoflurane. Thrombi were induced in mice with a strip of filter paper soaked in $10 \%-\mathrm{AlCl}_{3}$ which was applied to the right carotid artery $(R C A){ }^{[23]}$. After 5 minutes, the ${ }^{19} \mathrm{~F}-\mathrm{NP}-\mathrm{A} 14 \mathrm{CA}(\mathrm{n}=8)$ or ${ }^{19} \mathrm{~F}-\mathrm{NP} \mathrm{CA}(\mathrm{n}=6)$ was injected via the tail vein $(6.67 \mu \mathrm{l} / \mathrm{g}$ body weight). Ten minutes after thrombus induction, the filter paper was removed and the RCA was rinsed with saline.

Imaging. For in vivo MRI, mice were kept anesthetized using 1.5-2.0\% isoflurane in medical air and were placed in prone position in a dedicated animal holder with a built-in mask for anesthesia gas supply. A heating pad was placed over the mice to maintain normothermic conditions. Respiration rate and body temperature were continuously monitored with an MR compatible small animal monitoring system (SA Instruments, Inc., Stony Brook, NY, USA). Axial and coronal ' H 2D Fast Spin Echo (FSE) images were acquired to plan a sagittal slice package in plane of the carotid arteries (TE 9 ms; TR 1500 ms; acceleration factor 4; NSA 4; acquired and reconstructed pixel

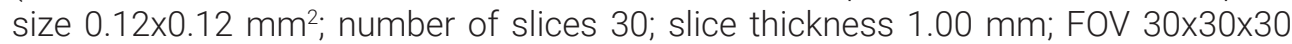
$\mathrm{mm}^{3}$ ). All ${ }^{1} \mathrm{H}$ acquisitions were gated to respiratory motion. Subsequently, the channel was switched and tuned to fluorine (282 MHz) MRI. A sagittal 2D gradient echo (GE) acquisition (TE 2.66 ms; TR 111 ms; NSA 24; flip angle (FA) 40; acquired 
and reconstructed pixel size $0.47 \times 0.47 \mathrm{~mm}^{2}$, number of slices 15 ; slice thickness

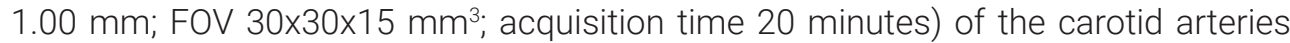
was performed with the same orientation as the ${ }^{1} \mathrm{H}$ acquisition. A pointed-resolved spectroscopy (PRESS) voxel (TE 14 ms; TR 1000 ms; NSA 1000; acquired voxel size 2x2x6 mm; acquisition time 17 minutes) was positioned on the RCA (thrombus side) and at the control side in the left carotid artery (LCA) at the same level as in the RCA.

\section{Ex vivo thrombus experiment}

Model. Excised carotid arteries were imaged to obtain high resolution and relatively high signal-to-noise fluorine images. Two hours after contrast injection, mice were sacrificed by an i.p. injection of pentobarbital $(100 \mathrm{mg} / \mathrm{kg})$. The RCA and LCA were excised, washed in saline, and embedded in $2 \%$ agarose gel.

Imaging. A ${ }^{1} \mathrm{H}$ scout scan was performed of the excised carotid arteries and subsequently a ${ }^{19} \mathrm{~F} 2 \mathrm{D}$ gradient echo MR pulse sequence was run for 12 hours. (TE 2.65 ms; TR 1000 ms; NSA 900; acquired and reconstructed pixel size $0.47 \times 0.47 \mathrm{~mm}^{2}$, slice thickness $1.00 \mathrm{~mm}$; number of slices 30; field of view $30 \times 30 \times 5 \mathrm{~mm}$ ).

\section{Image analysis}

To quantify the relative amount of fluorine in an image, an analysis based on the percentage of enhanced voxels was performed on the in-vitro and ex-vivo image data. In a fluorine image, a voxel was categorized as enhanced voxel (EV) when its signal intensity (SI) was higher than the mean plus two times the standard deviation of the background SI. For the analysis of fluorine images, two regions of interests (ROIs) were drawn. The first ROI encompassed the blood clot, and the second ROI was positioned completely in the background (agarose gel). Within the ROI of the thrombus, the percentage of EVs was determined. The percentages of EVs were compared between the two groups with different contrast agents using a Student's t-test. A p-value < 0.05 was considered statistically significant. For the ex vivo thrombus model also the signal-to-noise ratio (SNR) of the EVs and the background region was calculated.

\section{Blood plasma half-life}

In two animals, the plasma half-life of the ${ }^{19} \mathrm{~F}-\mathrm{NP}-\mathrm{A} 14 \mathrm{CA}$ was determined. In these animals, a thrombus was induced in the RCA as described above. After a scout scan, axial ${ }^{1} \mathrm{H}$ 2D FSE images were acquired for anatomical imaging (TE 9 ms; TR 1500 ms; acceleration factor 4; NSA 4; acquired and reconstructed voxel size $0.12 \times 0.12$

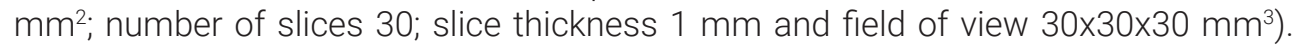
Subsequently, the channel was switched and tuned to fluorine MRI for the acquisition of a dynamic 2D axial GE acquisition (TE $2.66 \mathrm{~ms}$; TR $75 \mathrm{~ms}$; acquired and reconstructed voxel size $0.47 \times 0.47 \mathrm{~mm}^{2}$; slice thickness $3 \mathrm{~mm}$; number of slices 10 and field of view $30 \times 30 \times 30 \mathrm{~mm}$; NSA 32; 60 phases; acquisition time 1:55 minutes per phase). The slice package was positioned in the head-neck region. During this acquisition, $6.67 \mu \mathrm{l} / \mathrm{g}$ body weight of ${ }^{19} \mathrm{~F}-\mathrm{NP}$-A 14 CA was administered in the tail vein with an infusion pump, 9 minutes and 35 seconds (i.e. 5th dynamic phase) after the start of the acquisition. The blood plasma half-life was calculated by analyzing the signal intensity in the left 
Chapter 5

jugular vein. A mono-exponential decay was fitted through the signal intensity time curve for each mouse. 


\subsection{Results}

\section{CA characterization}

The hydrodynamic diameters determined from the DLS measurements of the ${ }^{19} \mathrm{~F}-\mathrm{NP}-\mathrm{A} 14$ and ${ }^{19} \mathrm{~F}-\mathrm{NP}$ CA were $281 \mathrm{~nm}$ and $272 \mathrm{~nm}$ with a polydispersity index (PDI) of 0.14 and 0.16 , respectively. The relaxation times $T_{1}$ and $T_{2}$ of the diluted ${ }^{19} \mathrm{~F}-\mathrm{NP} \mathrm{CA}$ in saline and in venous blood are given in Table 5.1. The $T$, increased from $696 \mathrm{~ms}$ for the CA nanoparticle in saline to $1272 \mathrm{~ms}$ when it was diluted in blood. Also the $T_{2}$ of the CA increased when it was diluted in blood from $294 \mathrm{~ms}$ to $524 \mathrm{~ms}$.

Table 5.1 Fluorine relaxation times of the pure contrast agent dissolved in either saline or venous blood. Data are presented as value \pm fit error.

\begin{tabular}{lll}
\hline & \multicolumn{1}{c}{ CA in saline $\mathbf{1 0 \%}(\mathrm{v} / \mathrm{v})$} & \multicolumn{1}{c}{ CA in blood $\mathbf{1 0 \%}(\mathrm{v} / \mathrm{v})$} \\
\hline${ }^{19} \mathrm{~F} T_{1}(\mathrm{~ms})$ & $696 \pm 70$ & $1272 \pm 352$ \\
${ }^{19} \mathrm{~F} T_{2}(\mathrm{~ms})$ & $294 \pm 8.2$ & $524 \pm 65$ \\
\hline
\end{tabular}

\section{In vitro imaging}

More ${ }^{19} \mathrm{~F}-\mathrm{NP}-\mathrm{A} 14 \mathrm{CA}$ was taken up by in vitro plasma clots than ${ }^{19} \mathrm{~F}-\mathrm{NP}$ CA (Figure 5.2). For the ${ }^{19} \mathrm{~F}-\mathrm{NP}-\mathrm{A} 14 \mathrm{CA}$ the rim of the clot shows many EVs compared to the center of the clot, whereas for the ${ }^{19} \mathrm{~F}-\mathrm{NP}$ CA the number of EVs is substantially lower and not confined to the rim of the clot. A larger percentage $(p<0.01)$ of EVs was found for the ${ }^{19} \mathrm{~F}-\mathrm{NP}-\mathrm{A} 14 \mathrm{CA}(16 \pm 1.0 \%$, mean $\pm \mathrm{SE})$ than the ${ }^{19} \mathrm{~F}-\mathrm{NP}$ CA $(7.2 \pm 1.2 \%)$ (Figure 5.2B).

\section{In vivo MR imaging}

In vivo imaging of clots was attempted using CA assisted fluorine MRI of fresh carotid artery clots (Figure 5.3). However, within the scan time of 20 minutes for the fluorine imaging and 17 minutes for each spectroscopic voxel, no clear fluorine signal was found in any carotid artery. Therefore, no further image analysis was performed. The signal intensity from the clot was not strong enough to discern it clearly from the background noise.

\section{Blood plasma half-life}

Analysis of the signal intensity time courses resulted in mono-exponential fluorine plasma half-lifes of 122 minutes $\left(R^{2}=0.34\right)$ and 74 minutes $\left(R^{2}=0.69\right)$ for the ${ }^{19} \mathrm{~F}-\mathrm{NP}-\mathrm{A} 14$ and ${ }^{19} \mathrm{~F}-\mathrm{NP} \mathrm{CA}$, respectively.

\section{Ex vivo MR imaging}

After in vivo imaging, the carotid arteries were taken out and imaged (Figure 5.4). For the ${ }^{19} \mathrm{~F}-\mathrm{NP}$-A14 CA a significantly larger percentage of EVs were measured 2 hours after contrast administration than for the ${ }^{19} \mathrm{~F}-\mathrm{NP}$ CA: $6.4 \pm 1.5 \%$ EVs versus $2.7 \pm 0.7$ $\%$ EVs, respectively (Figure 5.4B) (mean \pm SE). The SNR of the EVs was $6.3 \pm 1.0$ and $5.2 \pm 1.1$ for the ${ }^{19} \mathrm{~F}-\mathrm{NP}-\mathrm{A} 14$ and ${ }^{19} \mathrm{~F}-\mathrm{NP} \mathrm{CA}$, respectively (mean $\pm \mathrm{SE}$ ), illustrating that the ${ }^{19} \mathrm{~F}-\mathrm{NP}-\mathrm{A} 14 \mathrm{CA}$ specifically bound to the fresh clot. 


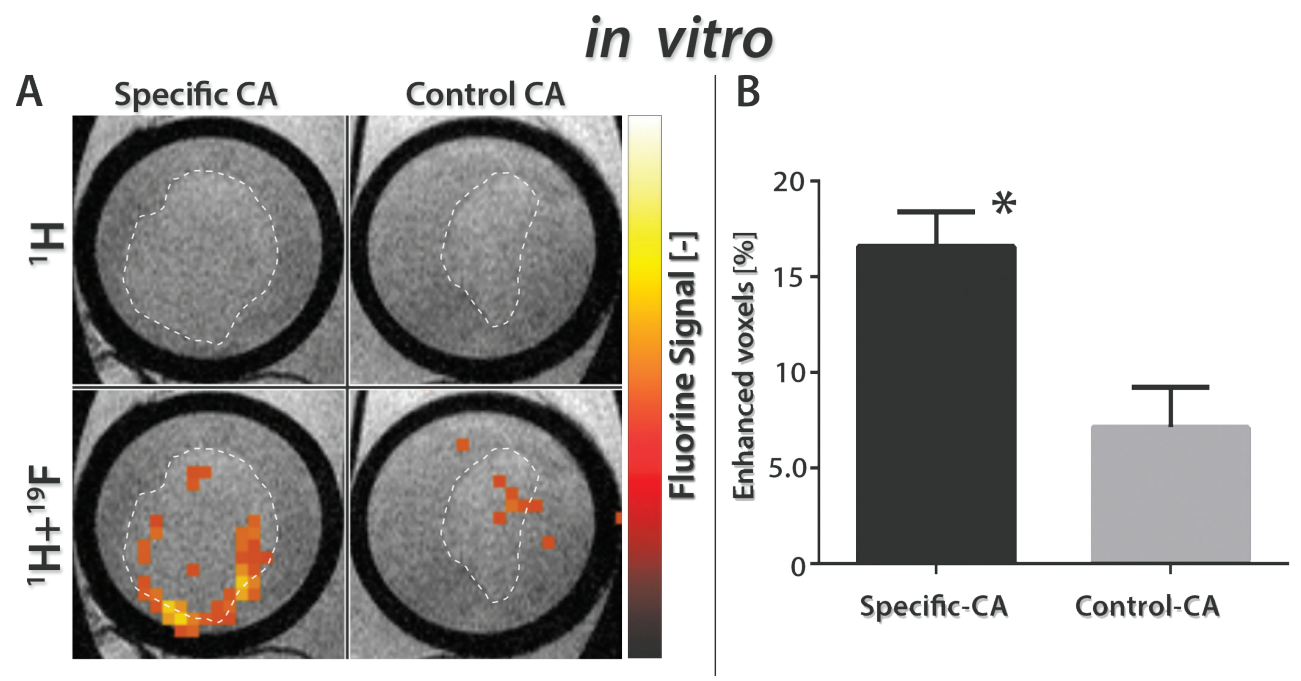

Figure 5.2 - A) MR images of an in vitro thrombus model embedded in agarose gel. Clots are delineated by the white dashed line. Top row shows the ${ }^{1} \mathrm{H}$ images and bottom row the same ${ }^{1} \mathrm{H}$ images with the ${ }^{19} \mathrm{~F}$ color overlay. The "hot iron" color scale shows fluorine signal intensity. The accumulation of the ${ }^{19} \mathrm{~F}-\mathrm{NP}-\mathrm{A} 14 \mathrm{CA}$ is typically observed at the rim of the thrombus. B) Contrast agent uptake, expressed as percentage of enhanced voxels A significantly higher percentage of enhanced voxels was found for the ${ }^{19} \mathrm{~F}-\mathrm{NP}-\mathrm{A} 14 \mathrm{CA}(\mathrm{n}=3)$ compared to the ${ }^{19} \mathrm{~F}$ NP CA $(n=3)(* P<0.01)$ (Data are presented as mean \pm one standard error).
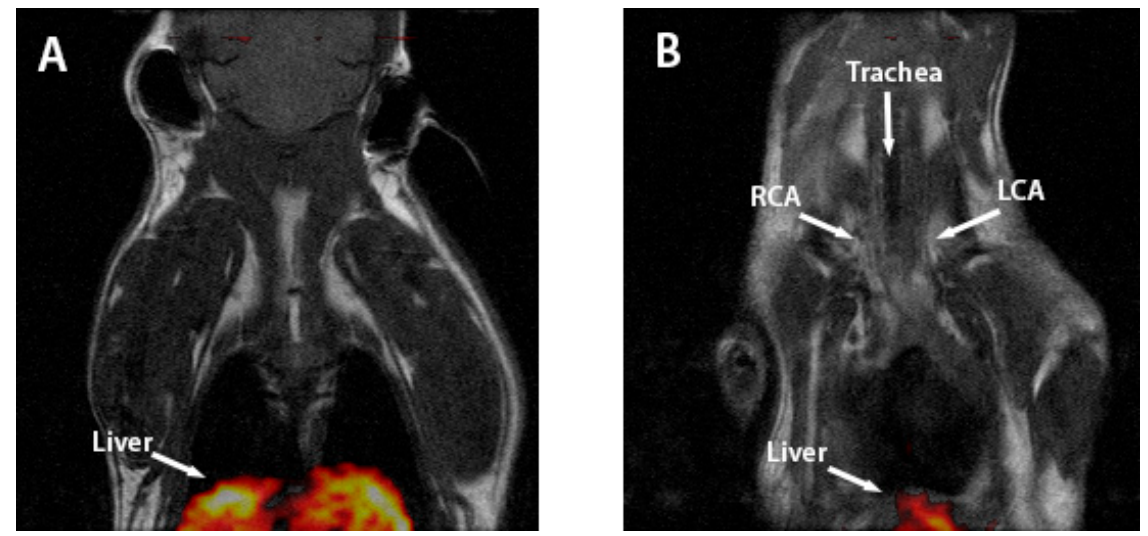

Figure 5.3 - A typical example of a coronal MR image in the superior part of the liver (A) and carotid arteries (B). The fluorine acquisition is projected as a color overlay on the anatomic image. In both images $A$ and $B$, clear accumulations of the fluorine contrast are found in the liver. In image B, the trachea and carotid arteries (RCA and LCA) are shown, but no clear fluorine signal could be detected for the right carotid artery with thrombus. 


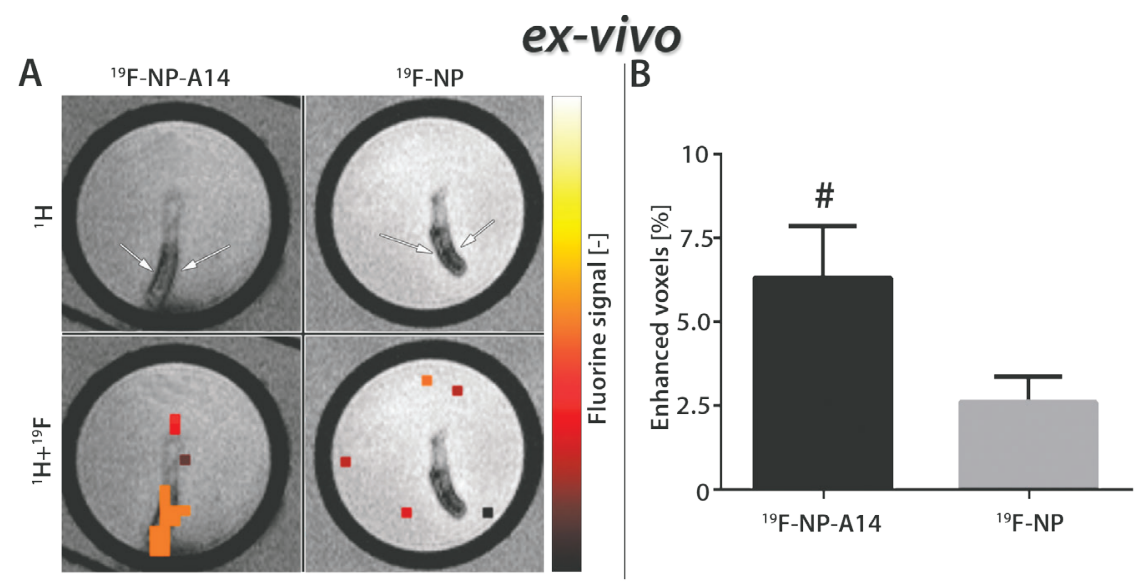

Figure 5.4 - Ex vivo MR images of the excised RCA with the induced thrombus (arrows) was embedded in agarose gel (A). The top row shows the ${ }^{1} \mathrm{H}$ images and the bottom row the ${ }^{1} \mathrm{H}$ images with the ${ }^{19} \mathrm{~F}$ color overlay. The "hot iron" color scale shows fluorine signal intensity in the image and depicts fluorine accumulation in the thrombus for the ${ }^{19} \mathrm{~F}-\mathrm{NP}-\mathrm{A} 14$ $\mathrm{CA}$, indicating binding of the ${ }^{19} \mathrm{~F}-\mathrm{NP}-\mathrm{A} 14 \mathrm{CA}$ to the in vivo induced thrombus. For the ${ }^{19} \mathrm{~F}-\mathrm{NP}$ $\mathrm{CA}$, the fluorine signal cannot be confined to the region of the thrombus. Uptake expressed as percentage of EVs, in the ex vivo thrombi (B). Significantly more uptake was found for the ${ }^{19} \mathrm{~F}-\mathrm{NP}-\mathrm{A} 14 \mathrm{CA}(\mathrm{n}=8)$ compared to the ${ }^{19} \mathrm{~F}-\mathrm{NP}$ CA $(\mathrm{n}=6)(\# \mathrm{P}<0.05)$ (Data are presented as mean \pm one standard error). 


\subsection{Discussion}

\section{Main findings}

This study presents a novel fluorine loaded polymeric NP based on PLGA-PEG decorated with A14 peptide for molecular MRI of thrombi. In vitro ${ }^{19} \mathrm{~F} \mathrm{MRI}$ of thrombi showed a larger percentage of enhanced voxels for the ${ }^{19} \mathrm{~F}-\mathrm{NP}-\mathrm{A} 14 \mathrm{CA}$ compared to the ${ }^{19} \mathrm{~F}-\mathrm{NP}$ CA. The same effect was found in the ex vivo images of the excised carotid arteries with artificially induced thrombi. In vivo MRI demonstrated uptake of contrast agent by the liver, however no contrast could be detected in the thrombus within the carotid artery.

\section{Properties of CA}

Both the ${ }^{19} \mathrm{~F}-\mathrm{NP}-\mathrm{A} 14$ and ${ }^{19} \mathrm{~F}-\mathrm{NP}$ CA have approximately the same size and polydispersity index. The comparable size and distribution indicate a consistent preparation of the two CAs. The $T_{1}$ relaxation time of $1272 \mathrm{~ms}$ measured in saline compared well with the value of $1400 \mathrm{~ms}$ of the phospholipids encapsulated perfluoro-15-crown-5-ether nanoparticles reported by van Heeswijk et al ${ }^{[24]}$. Also the $T_{2}$ relaxation time (524 ms) that was measured in the present study compares reasonably well with the latter study (440 ms in saline). However in venous blood, in contrast to our findings, van Heeswijk et al reported a comparable $T_{1}$ relaxation time $(1350 \mathrm{~ms})$ and a strong decrease in $T_{2}$ relaxation time ( $25 \mathrm{~ms})$, due the presence of (paramagnetic) hemoglobin. Differences in magnetic field strength, concentration and nanoparticles might explain, to some extent, to different results, but a full explanation for the observed differences in $T_{2}$ relaxation in venous blood cannot be given at this point.

\section{In vitro thrombus experiment}

The fibrin specific ${ }^{19} \mathrm{~F}-\mathrm{NP}-\mathrm{A} 14 \mathrm{CA}$ showed more uptake, mainly confined to the rim of the thrombus, than the ${ }^{19} \mathrm{~F}-\mathrm{NP}$ CA formulation. Both the larger percentage of enhanced voxels and the location of the uptake, emphasize the fibrin specific binding of ${ }^{19} \mathrm{~F}-\mathrm{NP}-\mathrm{A} 14 \mathrm{CA}$. As the CA was added after formation of the plasma clot, it could most likely only interacted with the rim of the thrombus, and not or much less with the interior of the thrombus.

\section{In vivo thrombus experiment}

Fluorine contrast agent was detected in the liver, indicating a successful contrast administration and circulation. Within an acquisition time of 20 minutes per measurement, we were not able to detect fluorine CA in the carotid arteries with fluorine MR imaging or spectroscopy, despite the relatively long plasma half-life. For spectroscopy, the voxel might be too large in comparison to the extent of fluorine uptake (partial volume signal loss). For imaging, this finding contrasted with the ex vivo results, which did reveal the presence of fluorine in the excised arteries with a considerably longer acquisition time of 12 hours. Increasing the in vivo acquisition time in future studies, might enable the detection of the ${ }^{19} \mathrm{~F}$ MR signal in the thrombi in vivo. However, long acquisition times are impractical, especially for studies that aim for clinical translation where timing of diagnosis and intervention is critical. 
Myerson et al studied the localized effect of a nanoparticle sized thrombin inhibitor as anticoagulant ${ }^{[25]}$. The thrombin inhibitor PPACK (Phe[D]-Pro-Arg-Chloromethylketone) was covalently linked to this nanoparticle and was assumed to bind to thrombin in a multivalent way. This nanoparticle was also fluorine loaded and the fluorine core enabled them to detect the nanoparticle with fluorine MRI in an ex-vivo thrombus. For that experiment an 11.7T MRI system was used. Within an acquisition time of 6 hours, they were able to visualize the uptake of the nanoparticles. In our ex-vivo experiment we doubled this acquisition time to compensate for the lower magnetic field strength (inherently lower signal-to-noise). Both experiments show that a long acquisition time is necessary to measure bound fluorine nanoparticles in a clot. So far, only Temme et al has successfully demonstrated the active targeted uptake of nanoparticles in vivo with fluorine MRI. They targeted fluorine nanoparticles to acute deep venous thrombi and pulmonary thromboemboli. As an example of non-active targeting, Flogel et al showed that the detection of phagocytosed fluorine loaded nanoparticles is feasible in vivo in inflammatory tissue with fluorine MRI [13]. This study also showed the uptake of the CA in the liver due to phagocytosis, which is well measureable in vivo.

\section{Ex vivo thrombus experiment}

The ex vivo measurements of the excised carotid arteries showed particle uptake in the thrombi from animal groups injected with the ${ }^{19} \mathrm{~F}-\mathrm{NP}-\mathrm{A} 14 \mathrm{CA}$ in comparison to the ${ }^{19} \mathrm{~F}-\mathrm{NP} \mathrm{CA}$, which is in line with the in vitro measurements. A reason for absence of the fluorine signal in vivo might be the partial volume effect for the in vivo measurement, which was much larger compared to the ex vivo measurements reducing the relative SNR/voxel with a factor 85 for the PRESS measurements, as calculated in the Appendix in this chapter. The low SNR of 6.26 , which was measured ex vivo means that the fluorine could not have been measured in vivo with the PRESS measurements.

\section{Outlook}

Molecular fluorine MR contrast agents could provide a number of advantages for molecular MRI. The absence of fluorine background signal is unique over other MR CAs. The present study focused on a novel CA for fresh thrombus targeting. CAs loaded with fluorine molecules could be a prelude to multi-spectral imaging of multiple biomarkers since different ${ }^{19} \mathrm{~F}$ compounds can be differentiated with MRI. CAs that visualize the thrombus in general and assess the susceptibility to fibrinolytic therapy could be highly beneficial in clinical practice. In the present study, we demonstrated the use of targeted fluorine loaded polymer nanoparticles with a single CA administration. The fluorine detection limit is still a big challenge for passive and active fluorine NP detection in in vivo MRI. Therefore, multiple CA injections over time are used to increase the amount of fluorine at the target area ${ }^{[11,13]}$. For clinical translation, the CA localization and binding efficiency should be high enough to detect a single administered dose. Besides CA optimizations to increase the SNR, also hardware configurations could increase the fluorine sensitivity ${ }^{[26]}$.

In conclusion, this study demonstrates the proof of concept for a novel polymeric molecular fluorine MRI CA that targets fresh thrombi. For this proof-of-concept study, the agent was targeted to thrombi. In the in vivo measurements the thrombi-targeted 
Chapter 5

fluorine was unfortunately below the detection limit of the experiment, although the fluorine CA could be detected in the ex vivo thrombi analysis. Polymeric nanoparticles might have potential for other molecular MR applications when the sensitivity of MRI is further increased. 


\section{Appendix, In vivo versus ex vivo MRI}

In the in vivo PRESS measurements, no fluorine signal was measured, where in the ex vivo Gradient Echo measurement the uptake of the fluorine CA was detectable. A few factors, e.g. relative SNR per voxel, signal loss by relaxation effects, or partial volume effects, can explain the difference results obtained by these measurements.

\section{Relative SNR}

The relative SNR per voxel for both MR sequences can be calculated with,

$$
\text { SNR/voxel }=\frac{\Delta x \Delta y \Delta z \sqrt{N S A}}{\sqrt{\frac{B W}{N_{x} N_{y} N_{z}}}}
$$

In this equation $\Delta x, \Delta y$ and $\Delta z$ are the voxel sizes and $N_{x^{\prime}} N_{y}$ and $N_{z}$ are the number of sample points in k-space. The relative $S N R /$ voxel for the two acquisitions are 17.2 and 16.7 for the in vivo (PRESS) and ex vivo (Gradient Echo) sequences, respectively.

\section{Signal loss by relaxation effects}

Due to the relative long $T_{2}$ compared the TE we can neglect signal losses due to dephasing related effects of the transverse magnetization. For the longitudinal magnetization a signal loss (incomplete recovery) can be calculated for the spin echo sequence for PRESS of $45.6 \%$ with a TR of $1000 \mathrm{~ms}$ and a $T_{1}$ of $1272 \mathrm{~ms}$. For the gradient echo sequence of the ex vivo measurement the loss is $16.4 \%$ with the same TR and $T_{1}$. Despite these signal losses, do the corresponding numbers still do not explain why the fluorine was not measurable in vivo.

\section{Partial volume effects}

The SNR/voxel calculated so far, assumes a homogeneous fluorine concentration in the voxel but this was not the case. The actual thrombus was small in comparison to the voxel size for the PRESS sequence. In the ${ }^{1} \mathrm{H}$ ex vivo images we could estimate that the thrombus had a diameter of approximately 3 voxels $(0.36 \mathrm{~mm})$ and a length of approximately 18 voxels $(2.16 \mathrm{~mm})$. This means that the partial volume effect for the in vivo measurement was much larger than for the ex vivo measurement and will reduce the relative SNR/voxel with approximately a factor of 85 for the PRESS measurements.

The partial volume effect gives by far the largest contribution to the differences between the in vivo and ex vivo measurements. With an SNR of 6.26 which was measured ex vivo this means that the in vivo SNR would be below 0.1 , which is simply not detectable 


\section{References}

1. Ciesienski, K.L. and P. Caravan, Molecular MRI of Thrombosis. Curr Cardiovasc Imaging Rep, 2010. 4(1): p. 77-84.

2. Appleby, P.B., C; Collins, R; Flather, M; et al, Indications for fibrinolytic therapy in suspected acute myocardial infarction: collaborative overview of early mortality and major morbidity results from all randomised trials of more than 1000 patients. Fibrinolytic Therapy Trialists' (FTT) Collaborative Group. Lancet, 1994. 343(8893): p. 311-22.

3. Goldstein, L.B., Acute ischemic stroke treatment in 2007. Circulation, 2007. 116(13): p. 1504-14.

4. Lange, R.A. and L.D. Hillis, Reperfusion therapy in acute myocardial infarction. N Engl J Med, 2002. 346(13): p. 954-5.

5. Botnar, R.M., A. Buecker, A.J. Wiethoff, E.C. Parsons, Jr., M. Katoh, G. Katsimaglis, R.M. Weisskoff, R.B. Lauffer, P.B. Graham, R.W. Gunther, W.J. Manning, and E. Spuentrup, In vivo magnetic resonance imaging of coronary thrombosis using a fibrin-binding molecular magnetic resonance contrast agent. Circulation, 2004. 110(11): p. 1463-6.

6. Spuentrup, E., R.M. Botnar, A.J. Wiethoff, T. Ibrahim, S. Kelle, M. Katoh, M. Ozgun, E. Nagel, J. Vymazal, P.B. Graham, R.W. Gunther, and D. Maintz, MR imaging of thrombi using EP2104R, a fibrin-specific contrast agent: initial results in patients. Eur Radiol, 2008. 18(9): p. 1995-2005.

7. Uppal, R., K.L. Ciesienski, D.B. Chonde, G.S. Loving, and P. Caravan, Discrete bimodal probes for thrombus imaging. J Am Chem Soc, 2012. 134(26): p. 10799-802.

8. Miserus, R.J., M.V. Herias, L. Prinzen, M.B. Lobbes, R.J. Van Suylen, A. Dirksen, T.M. Hackeng, J.W. Heemskerk, J.M. van Engelshoven, M.J. Daemen, M.A. van Zandvoort, S. Heeneman, and M.E. Kooi, Molecular MRI of early thrombus formation using a bimodal alpha2-antiplasmin-based contrast agent. JACC Cardiovasc Imaging, 2009. 2(8): p. 98796.

9. Caruthers, S.D., A.M. Neubauer, F.D. Hockett, R. Lamerichs, P.M. Winter, M.J. Scott, P.J. Gaffney, S.A. Wickline, and G.M. Lanza, In vitro demonstration using ${ }^{19} \mathrm{~F}$ magnetic resonance to augment molecular imaging with paramagnetic perfluorocarbon nanoparticles at 1.5 Tesla. Invest Radiol, 2006. 41(3): p. 305-12.

10. Partlow, K.C., J. Chen, J.A. Brant, A.M. Neubauer, T.E. Meyerrose, M.H. Creer, J.A. Nolta, S.D. Caruthers, G.M. Lanza, and S.A. Wickline, ${ }^{19} \mathrm{~F}$ magnetic resonance imaging for stem/ progenitor cell tracking with multiple unique perfluorocarbon nanobeacons. FASEB J, 2007. 21(8): p. 1647-54.

11. Temme, S., C. Grapentin, C. Quast, C. Jacoby, M. Grandoch, Z. Ding, C. Owenier, F. Mayenfels, J.W. Fischer, R. Schubert, J. Schrader, and U. Flogel, Noninvasive Imaging of Early Venous Thrombosis by ${ }^{19} \mathrm{~F}$ Magnetic Resonance Imaging With Targeted Perfluorocarbon Nanoemulsions. Circulation, 2015. 131(16): p. 1405-14.

12. Flogel, U., Z. Ding, H. Hardung, S. Jander, G. Reichmann, C. Jacoby, R. Schubert, and J. Schrader, In vivo monitoring of inflammation after cardiac and cerebral ischemia by fluorine magnetic resonance imaging. Circulation, 2008. 118(2): p. 140-8.

13. Flogel, U., S. Su, I. Kreideweiss, Z. Ding, L. Galbarz, J. Fu, C. Jacoby, O. Witzke, and J. Schrader, Noninvasive detection of graft rejection by in vivo (19) F MRI in the early stage. Am J Transplant, 2011. 11(2): p. 235-44. 
14. Neubauer, A.M., J. Myerson, S.D. Caruthers, F.D. Hockett, P.M. Winter, J. Chen, P.J. Gaffney, J.D. Robertson, G.M. Lanza, and S.A. Wickline, Gadolinium-modulated ${ }^{19} \mathrm{~F}$ signals from perfluorocarbon nanoparticles as a new strategy for molecular imaging. Magn Reson Med, 2008. 60(5): p. 1066-72.

15. Bonner, F., C. Jacoby, S. Temme, N. Borg, Z. Ding, J. Schrader, and U. Flogel, Multifunctional MR monitoring of the healing process after myocardial infarction. Basic Res Cardiol, 2014. 109(5): p. 430.

16. Panyam, J. and V. Labhasetwar, Biodegradable nanoparticles for drug and gene delivery to cells and tissue. Adv Drug Deliv Rev, 2003. 55(3): p. 329-47.

17. Pinto Reis, C., R.J. Neufeld, A.J. Ribeiro, and F. Veiga, Nanoencapsulation I. Methods for preparation of drug-loaded polymeric nanoparticles. Nanomedicine, 2006. 2(1): p. 8-21.

18. Gupta, M., G. Chashoo, P.R. Sharma, A.K. Saxena, P.N. Gupta, G.P. Agrawal, and S.P. Vyas, Dual Targeted Polymeric Nanoparticles Based on Tumor Endothelium and Tumor Cells for Enhanced Antitumor Drug Delivery. Mol Pharm, 2014.

19. Liu, Y., K. Li, B. Liu, and S.S. Feng, A strategy for precision engineering of nanoparticles of biodegradable copolymers for quantitative control of targeted drug delivery. Biomaterials, 2010. 31(35): p. 9145-55.

20. Langereis, S., H.A. Kooistra, M.H. van Genderen, and E.W. Meijer, Probing the interaction of the biotin-avidin complex with the relaxivity of biotinylated Gd-DTPA. Org Biomol Chem, 2004. 2(9): p. 1271-3.

21. Wolters, M., M. Oostendorp, B.F. Coolen, M.J. Post, J.M. Janssen, G.J. Strijkers, M.E. Kooi, K. Nicolay, and W.H. Backes, Efficacy of positive contrast imaging techniques for molecular MRI of tumor angiogenesis. Contrast Media Mol Imaging, 2012. 7(2): p. 130-9.

22. Samadi, N., M.J. van Steenbergen, J.B. van den Dikkenberg, T. Vermonden, C.F. van Nostrum, M. Amidi, and W.E. Hennink, Nanoparticles based on a hydrophilic polyester with a sheddable PEG coating for protein delivery. Pharm Res, 2014. 31(10): p. 2593-604.

23. Wolters, M., R.H. van Hoof, A. Wagenaar, K. Douma, M.A. van Zandvoort, T.H. Hackeng, M.J. Post, W.H. Backes, and M.E. Kooi, MRI artifacts in the ferric chloride thrombus animal model: an alternative solution: preventing MRI artifacts after thrombus induction with a non-ferromagnetic Lewis acid. J Thromb Haemost, 2013. 11(9): p. 1766-9.

24. van Heeswijk, R.B., Y. Pilloud, U. Flogel, J. Schwitter, and M. Stuber, Fluorine-19 magnetic resonance angiography of the mouse. PLoS One, 2012. 7(7): p. e42236.

25. Myerson, J., L. He, G. Lanza, D. Tollefsen, and S. Wickline, Thrombin-inhibiting perfluorocarbon nanoparticles provide a novel strategy for the treatment and magnetic resonance imaging of acute thrombosis. J Thromb Haemost, 2011. 9(7): p. 1292-300.

26. Wolters, M., S.G. Mohades, T.M. Hackeng, M.J. Post, M.E. Kooi, and W.H. Backes, Clinical perspectives of hybrid proton-fluorine magnetic resonance imaging and spectroscopy. Invest Radiol, 2013. 48(5): p. 341-50. 


\section{Super-resolution}

\section{reconstruction techniques for}

${ }^{19}$ Fluorine MR angiography

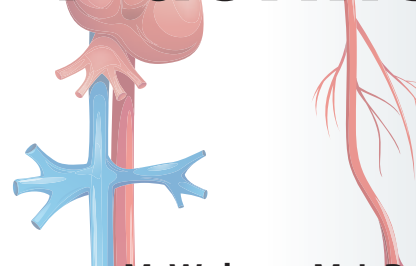

M. Wolters, M.J. Post, W.H. Backes, and M.E. Kooi

In submission

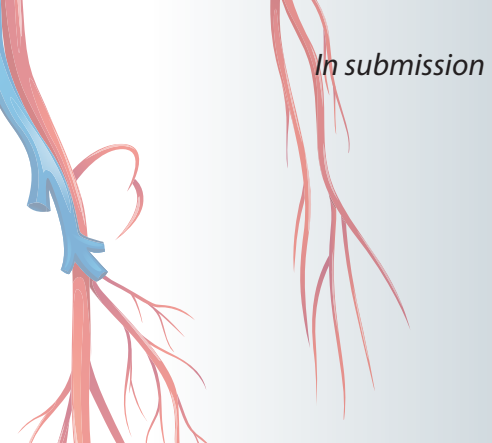


Purpose Fluorine $\left({ }^{19} \mathrm{~F}\right)$ magnetic resonance $(\mathrm{MR})$ angiography has the advantage of direct detection of the fluorine contrast medium, without the need to suppress the background tissue signal. However, fluorine MRI requires long acquisition times due to relatively slow $T_{1}$ relaxation and low contrast-to-noise levels. Superresolution reconstruction (SRR) techniques can shorten the acquisition time of high-spatial resolution MR images. This study aims to investigate the potential of three SRR techniques, i.e. Iterative Back Projection (IBP), Robust Iterative Back Projection (RIBP) and linear INTerpolation (INT).

Methods The image quality obtained with the three reconstruction algorithms is compared in a simulated phantom. The comparison is also made in a postmortem mouse model using native 3D MR angiography as a reference.

Results Each of the three SRR techniques has distinct advantages regarding specific image features. IBP has the best spatial resolution performance (FWHM 1.0), closely followed by RIBP (FWHM 1.1) which has also relatively good image contrast for imput images with a SNR $>8$. INT provides the best image contrast when the input images are rather noisy. However, at longer acquisition times, 3D MR angiography still showed more spatial details in the port-mortem fluorine MR angiogram.

Conclusion Under conditions where the acquisition is dominated by a low signalto-noise ratio, such as fluorine MR angiography, SRR methods can be beneficial over native $3 \mathrm{D}$ acquisitions. IBP and RIBP demonstrates a superior spatial resolution in comparision to INT. While the INT technique outweighed IBP and RIBP in image contrast. RIBP showed good trade-off in terms of spatial resolution and image contrast when the input images are less noisy. INT provides the highest gain in SNR, which is advantageous over native 3D MRI acquisitions by reduction of acquisition time. 


\subsection{Introduction}

Contrast-enhanced magnetic resonance (MR) angiography is widely applied to visualize the arteries and veins, since it is non-invasive, does not involve ionizing radiation, and can be easily combined with visualization of the surrounding soft-tissue anatomy.

Gadolinium enhanced MR angiography enhances the signal intensity on $T_{1}$ weighted MR images in the vessel lumen. However, the entire soft-tissue anatomy gives rise to the MR signal, including a high signal from fat and other tissues with short $T_{1}$ relaxation times, which contributes to a rather inhomogeneous background. Suppressing this background signal remains challenging for MR angiography ${ }^{[1]} .{ }^{19} \mathrm{~F}$-fluorine-based MR angiography may overcome this problem ${ }^{[2]}$, since it has the advantage that only the fluorine is depicted on the basis of its (nuclear) spin density, while the background tissue does not contain fluorine. Thus, fluorine MRI results in an image of the in vivo fluorine distribution without a background signal originating from other tissues. The direct visualization of the fluorine signal also allows the generation of an overlay of the fluorine distribution on the anatomical soft-tissue proton image. However, the signal of ${ }^{19} \mathrm{~F}-\mathrm{MRI}$ at a dose of fluorine that can acceptably be administered in vivo is rather low. Van Heeswijk et al reported a detection limit for in vitro ${ }^{19} \mathrm{~F} M R I$ of $\approx 400 \mathrm{mM}$ perfluoro-15-crown-5 ether (PCE) emulsion ${ }^{[3]}$.

A disadvantage of fluorine contrast medium (CM) is their relatively long and oxygenconcentration dependent $T_{1}$ relaxation time $\left(>1000 \mathrm{~ms}\right.$ below 3 Tesla) ${ }^{[4]}$. To obtain sufficient signal for $T_{1}$-weighted imaging and to eliminate the effect of the oxygen dependency, pulse sequences with a long repetition time (TR) are required to avoid magnetization saturation, thereby prolonging the total scan duration, especially for 3D MRI with high-spatial resolution.

High-resolution MR images with isotropic voxels and long TRs within acceptable acquisition times may be achieved with a super-resolution reconstruction (SRR) technique. For SRR, multiple, (2D) multi-slice imaging volumes need to be acquired instead of a 3D volume acquisition. Whereas one single $\mathrm{k}$-line is acquired in a 3D acquisition per TR, for SRR in a multi-slice acquisition a $\mathrm{k}$-line is acquired for each slice resulting in multiple k-lines per TR. This will accelerate the acquisition, but the slices need be thicker to compensate for the general loss of signal-to-noise in 2D imaging compared to 3D imaging. To improve the spatial resolution in the slice direction with SRR, sampling of the volume with multiple 2D acquisitions with a geometric off-set (translation or angulation) is performed ${ }^{[5-7]}$. SRR is thus a combination of a multiangular multi-slice acquisition and a post-processing technique that can produce a single high-resolution image based on a set of (angulated in case of MRI) lowresolution images. The SRR methods benefits from the shorter acquisition time of the multi-slice acquisition technique and therefore SRR acquisition strategies can 
be faster than $3 \mathrm{D}$ acquisitions for contrast media with a long $T_{1}$ relaxation time like fluorine.

There is a relationship between the number of input LR images and the HR image quality but it is unknown for fluorine MRI what would be the optimal number of angular LR images. Thus far, SRR has been applied for ${ }^{1} \mathrm{H}-\mathrm{MRI}$, especially for anatomical imaging $[6,8-10]$ and diffusion tensor imaging ${ }^{[11-16]}$. Yet it remains to be shown to which extent SRR methods can be used for fluorine MR angiography. Fluorine MRI generally suffers from a relatively low signal-to-noise ratio (SNR) and the effect of noisy images of fluorine imaging on SRR is unknown.

In the present study we aim to investigate the potential of three SRR techniques for fluorine MR angiography. We studied two iterative post-processing algorithms, i.e. Iterative Back Projection (IBP) introduced by Irani and Peleg [17, 18], Robust Iterative Back Projection (RIBP) proposed by Zomet et al ${ }^{[19]}$ and a third method based on linear INTerpolation (INT) by Vandewalle et al [20]. These methods were applied and reported in several studies in silico and in vivo for ${ }^{1} \mathrm{H}$ MRI ${ }^{[8,21-23]}$. We focus in this study on the use of these methods for ${ }^{19} \mathrm{~F}$ MR angiography, which usually deals with relatively noisy images. For a quantitative analysis, the images reconstructed by the three algorithms are analyzed on image quality, i.e. SNR, image error and spatial resolution in a simulated in silico phantom. The SRR methods are also applied for a qualitative analysis of a post-mortem small-animal model to depict fluorine-enhanced blood vessels and to compare with a native 3D MR angiogram. 


\subsection{Methods}

Concept of the Super-Resolution Reconstruction reverse problem SRR is a reverse problem, for which an estimate of a high resolution $(H R)$ image is calculated from a number of low resolution (LR) images. The concept of this reverse problem is depicted in the model given in Figure 6.1.

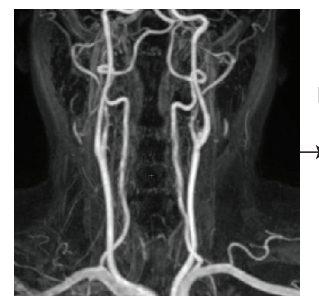

HR Image $x$

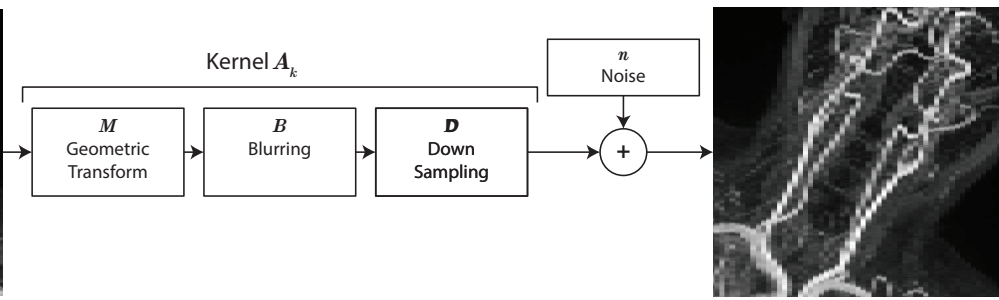

LR Image $y_{k}$

Figure 6.1 - Super-Resolution Reconstruction is a reverse problem in which from a high resolution (HR) image $x$, a series of low resolution (LR) images $y_{k}$ is obtained after applying the acquisition model $\boldsymbol{A}_{k}$ and adding noise $\boldsymbol{n}_{k}$. The acquisition model comprises a geometric transformation $(\boldsymbol{M})$, which is different for each LR image and is identified by index $k$, followed by blurring $(\boldsymbol{B})$ and spatial down-sampling $(\boldsymbol{D})$.

This model can only provide a solution for an LR image when the HR image is known. The input of the model is the HR image, which can be parameterized as a matrix $(x)$. The acquisition model subsequently performs a geometric transformation $(\boldsymbol{M})$, which may be any combination of translation and rotation, linear space-invariant blurring $(\boldsymbol{B})$ and a (linear) down-sampling $(\boldsymbol{D})$, taken together defined as $\boldsymbol{A}=\boldsymbol{D} \boldsymbol{B} \boldsymbol{M}{ }^{[21]}$. Finally, (acquisition) noise $(\boldsymbol{n})$ is added to the image $\left(\boldsymbol{A}_{x}\right)$ to obtain the LR image $\boldsymbol{y}$. For a series of geometric transformations $(k)$, comprising various translations and angulations, a series of LR images $\left(\boldsymbol{y}_{k}\right)$ is obtained. In matrix form this model can be denoted as

$$
y_{k}=A_{k} x+n_{k}
$$

Obtaining an exact solution from the reverse problem for $\boldsymbol{x}$, when only $\boldsymbol{y}$ is known, is not possible. The presence of noise and the unknown information in kernel $\boldsymbol{A}$ makes the model an ill-posed problem, as is described by Plenge et al [21] in more detail. To obtain an estimate for $\boldsymbol{x}$, iterative methods such as iterative back projection, algebraic reconstruction or least squares solving methods can be employed ${ }^{[5,21]}$.

\section{Super-resolution reconstruction algorithms}

To solve the image reconstruction problem, three different post-processing methods are used. Two of these methods are backprojection algorithms, while the third one is based on linear interpolation of the LR images. From the backprojection algorithms we expect a better spatial image resolution than for the linear interpolation algorithm, the latter being computationally faster because it lacks iterative steps. The two backprojection algorithms start with an initial guess of the HR image $\hat{x}^{l}$, based on an up-sampled version of the first acquired LR image. At each next iterative step, the error image between the LR image $\boldsymbol{y}_{k}$ and the previous HR image $\hat{x}^{l}$ is calculated by subtraction. This error image $\left(y_{k}-A_{k}\left(\hat{x}^{l}\right)\right)$ is processed by the backprojection kernel $\left(\boldsymbol{H}_{\boldsymbol{B P}}\right)$ and transformed (i.e. numerically added) to the previously calculated 
HR image. The backprojection kernel performs the inverse operation of kernel, by subsequently upsampling, sharpening, and (inverse) geometrically transforming the error images. For IBP, the error images calculated from the different $L R$ images are averaged, whereas for RIBP the median image of these error images are projected to the previous calculated HR image. The use of the median operation makes RIBP more robust against the presence of image noise than the IBP algorithm.

The first method, IBP, can be formulated as

$$
\hat{x}^{l+1}=\hat{x}^{l}+\sum_{k=1}^{N} H_{B P}\left(y_{k}-A_{k}\left(\hat{x}^{l}\right)\right)
$$

In equation 6.2, $\boldsymbol{H}_{B P}$ represents the backprojection kernel, performing the inverse operation of the kernel $\boldsymbol{A}$. Index $\boldsymbol{l}$ is the number of the iteration step. The pixel-by-pixel differences between the estimated and each LR image are summed and used as the error correction in the iteration loop.

For RIBP the median, instead of the mean, is used in the reconstruction algorithm, which makes it more robust to outliers ${ }^{[19]}$. The median of the difference of the backprojected images is taken and used as new input for the error correction iteration loop as

$$
\hat{x}^{l+1}=\hat{x}^{l}+N \cdot \operatorname{median}\left(H_{B P}\left(y_{k}-A_{k}\left(\hat{x}^{l}\right)\right)\right)_{k=1}^{N}
$$

Both iterative methods have been implemented as described by Vandewalle et al [20]

The third method, INT, is used to obtain the HR image by spatial interpolation. This method is not iterative and the HR image is created in a single computational step [22]. The HR image is obtained by summation of the LR images after application of the backprojection kernel $\left(\boldsymbol{H}_{B P}\right)$

$$
x=\sum_{k=1}^{N} H_{B P}\left(y_{k}\right)
$$

The kernel $\boldsymbol{H}_{B P}$ applies subsequently up-sampling by linear interpolation, sharpening, and (inverse) geometrically transforming over all the LR images. Interpolation of the set of $L R$ images is a relatively fast method to obtain the HR image with a relatively high SNR and little improvement in spatial resolution to be expected ${ }^{[20]}$.

\section{Computer simulations}

The three SRR methods were evaluated by means of a simulated 'in silico' 2D HR phantom image that comprised various geometric and contrast elements suitable for quantitative image quality evaluation. For the comparison of the three SRR methods, the SNR, the reconstruction error (i.e. the image error) and spatial resolution were studied. From the HR phantom image a number of LR images was calculated in a 
way that simulated the multi-angular multi-slice aspects of the image acquisition process, as described below. Hereafter, a HR image was reconstructed again with the previously described SRR methods. The HR reference image used for the simulation is shown in Figure 6.2 and contains a number of sections. 1) The large circular section A contains a set of quarter circles and also includes sections $B, C$ and $D$. This part is used to calculate the image error of the reconstruction. 2) The rectangular section $B$ (110x20 pixels) contains, a line with a thickness of 1 pixel and 100\% contrast is used to measure the full-width-at-half-maximum (FWHM) in the reconstructed images to quantify the spatial resolution. 3) The rectangular section $\mathrm{C}$ is used to measure the SNR in two circular regions with $100 \%$ and $0 \%$ contrast. 4) In the rectangular section D, two bar patterns with different contrast, $10 \%$ and $30 \%$, and increasing line width from 1 to 10 pixels are used to assess the low-contrast resolution.

SRR reconstructions were calculated from a series of $L R$ images from the HR reference image (Figure 6.2) by rotating $\left(-115,-95 . .+45^{\circ}\right)$, blurring, spatial downsampling and adding random noise (SNR of 2, 4, 8, 16 and 32), as schematically represented in Figure 6.1. Examples of LR images after rotating, blurring and spatial downsampling are shown in Figure 6.3.

\section{MRI acquisitions}

To compare the three SRR methods to a native 3D fluorine MR angiography acquisition, a number of 2D LR datasets for SRR and one 3D dataset as a HR reference were acquired in a postmortem mouse imaging experiment. Both acquisitions used a Fast Spin Echo (FSE) sequence for which the acquisition parameters are given in Table 6.1. Improving the image quality with a longer acquisition time was achieved by increasing the number of signal averages (NSA) or increasing the repetition time (TR), for the 2D and $3 \mathrm{D}$ acquisitions, respectively. For all 9 angulations an increment of 20 degrees was applied. In addition, a series of 5 images was acquired with different NSA, leading to a dataset of 45 LR 2D images for the SRR. For the 3D reference MR images, 7 acquisitions were performed with different TRs. These extensive 3D datasets were acquired to obtain a range of subsets for post-processing, with different acquisition times depending on the number of reconstruction angles, NSA, and a range of TR settings. The acquisition times for the subsets are given in Table 6.2.The SRR is applied to a set of angulated 2D image sets. An example of slice package positions relative to the object is shown in Figure 6.4. 


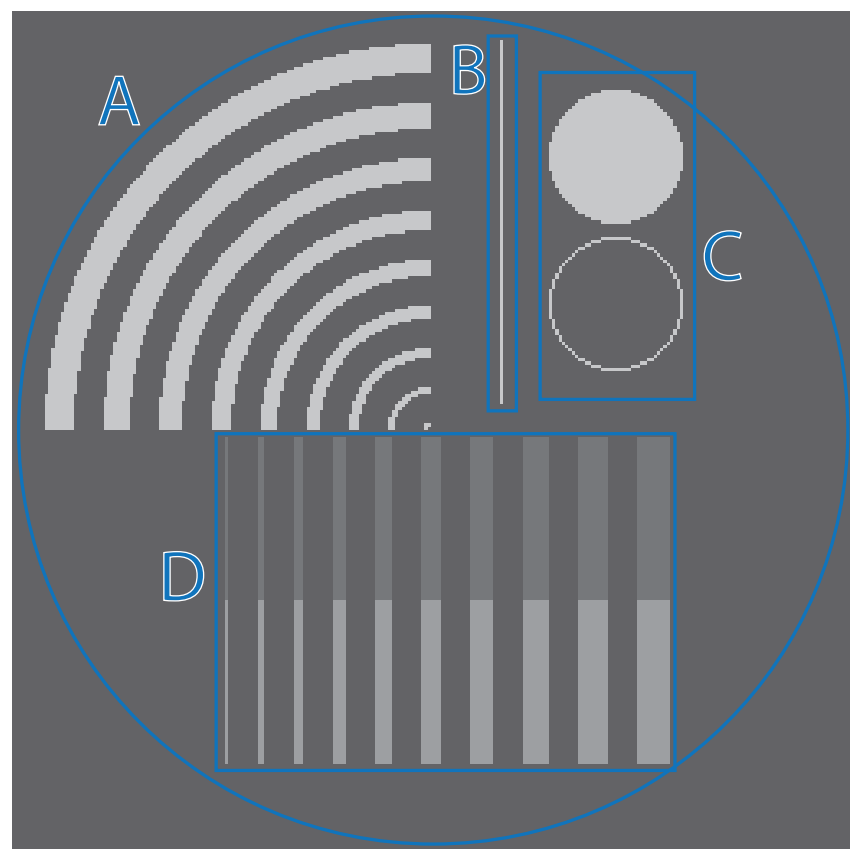

Figure 6.2 - High-resolution phantom image with a matrix size of $256 \times 256$ pixels for the computer simulations. This reference image contains the following sections: (A) A set of quarter circles with increasing radius and line width to contribute to the determination of the image error after the reconstruction. Note that the reconstruction image error is determined from the entire HR image, including all the sections A-D. (B) A 1 pixel thick line with $100 \%$ contrast to measure the spatial resolution. (C) Two circular regions with $100 \%$ and $0 \%$ contrast to measure signal-to-noise ratio. (D) Bar patterns with increasing line width from 1 to 10 pixels and two contrast settings, $10 \%$ and a $30 \%$, to measure the low-contrast resolution; bars are separated by 10 background pixels.

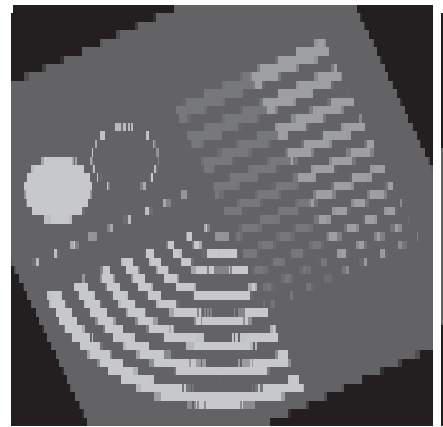

$-115^{\circ}$

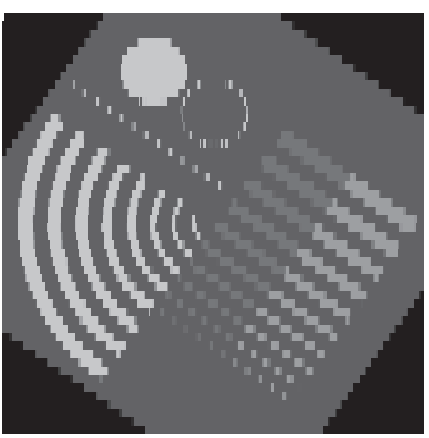

$-55^{\circ}$

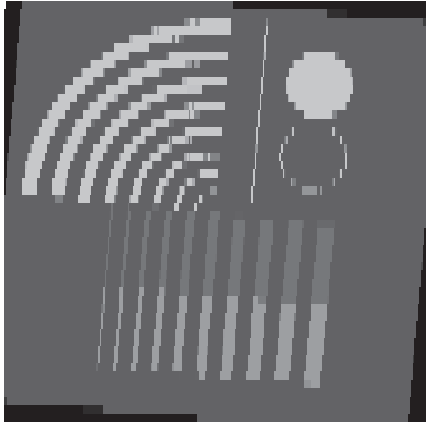

$5^{\circ}$

Figure 6.3 - Low resolution (LR) images of the phantom to simulate the multi-angle multislice acquisition process. LR images are shown at 3 angles after rotation, blurring, and spatial downsampling; noise was not yet added at this stage. Note that the downsampling resulted in rectangular pixels with an aspect ratio of 5:1. This pixel aspect ratio corresponds with the LR images obtained from the MR acquisitions, which have a slice thickness of $0.6 \mathrm{~mm}$ and pixel size of $0.12 \mathrm{~mm}$ in the phase-encoding direction. 
Table 6.1 - Acquisition parameters for 2D and 3D fluorine postmortem MR angiography.

\begin{tabular}{lcc}
\hline & 2D & 3D \\
\hline Echo time (TE) (ms) & 9 & 9 \\
Repetition time (TR) (ms) & 2100 & $50,100,200,300,400$, \\
& & $500,1000,2000$ \\
Acceleration (RARE) factor & 4 & 4 \\
Voxel size (mm) & $0.12 \times 0.12$ & $0.12 \times 0.12 \times 0.12$ \\
Slice thickness (mm) & 0.6 & - \\
Number of slices & 50 & $256 \times 256 \times 192$ \\
Acquisition matrix size & $256 \times 192$ & - \\
Reconstruction matrix size & $256 \times 256$ & 1 \\
Angulation $\left(^{\circ}\right)^{*}$ & $-115,-95, \ldots 45$ & - \\
Signal averages (NSA) & $1,2,4,8,16$ & \\
* For the rotation axis the middle line of the slice package was used, perpendicular to \\
the high-resolution image plane. & &
\end{tabular}

Table 6.2 - Acquisition times for 2D subsets (number of signal averages, 1 ) and 3D (repetition time, $50 \mathrm{~ms}$ ) MRI.

\begin{tabular}{cc}
\hline Datasets & Acquisition time (min:sec) \\
\hline 2D 3 angles $\left(-115,-55,5^{\circ}\right)$ & $4: 57$ \\
2D 5 angles $\left(-115,-75,-35,5,45^{\circ}\right)$ & $8: 15$ \\
2D 9 angles $\left(-115,-95,-75,-55,-35,-15,5,25,45^{\circ}\right)$ & $14: 51$ \\
3D & $10: 14$ \\
\hline
\end{tabular}

\section{Contrast media preparation}

The fluorine CM was prepared by dissolving PLGA-PEG ( poly(lactic-co-glycolic acid)-Polyethylene glycol) (3 mg) and PLGA (30 mg) in dichloromethane (1 ml) and methanol $(333 \mu \mathrm{l})$. Perfluoro-15-crown-5-ether $(\mathrm{CE})(100 \mu \mathrm{l})$ was emulsified in the polymer solution. Subsequently, an aqueous $0.5 \%$ polyvinyl alcohol buffer solution $(5 \mathrm{ml})$ was added and sonicated for 60 seconds with a tip-sonicator (MSE Mark II). Afterwards, $33 \mathrm{ml}$ of $0.5 \%$ polyvinyl alcohol buffer was added and stirred overnight to evaporate the solvents. The nanoparticles solution was centrifuged at $100 \mathrm{~g}$ to obtain pellets, which were dissolved in saline with a final volume of $1 \mathrm{ml}$ for each batch.

\section{Post-mortem imaging experiment}

A Swiss mouse was euthanized using carbon dioxide gas. Directly after death, the mouse was perfused with the CM. Perfusion was performed with infusion lines placed in the aorta and vena cava. First, saline flushing was performed until nearly all blood was washed out. Second, the CM was administered in the aorta through the infusion line. Hereafter, the animal was placed in the MRI system.

Scout images were acquired using a ${ }^{1} \mathrm{H}$ 2D Fast Spin Echo (FSE) sequence. Proton MR images were obtained in three orthogonal directions (TE 9 ms; TR 1500 ms; RARE factor 4; acquired voxel size $0.12 \times 0.12 \times 1.00 \mathrm{~mm}$, FOV $30 \times 30 \times 30 \mathrm{~mm}$ ). For the ${ }^{19} \mathrm{~F}$ 


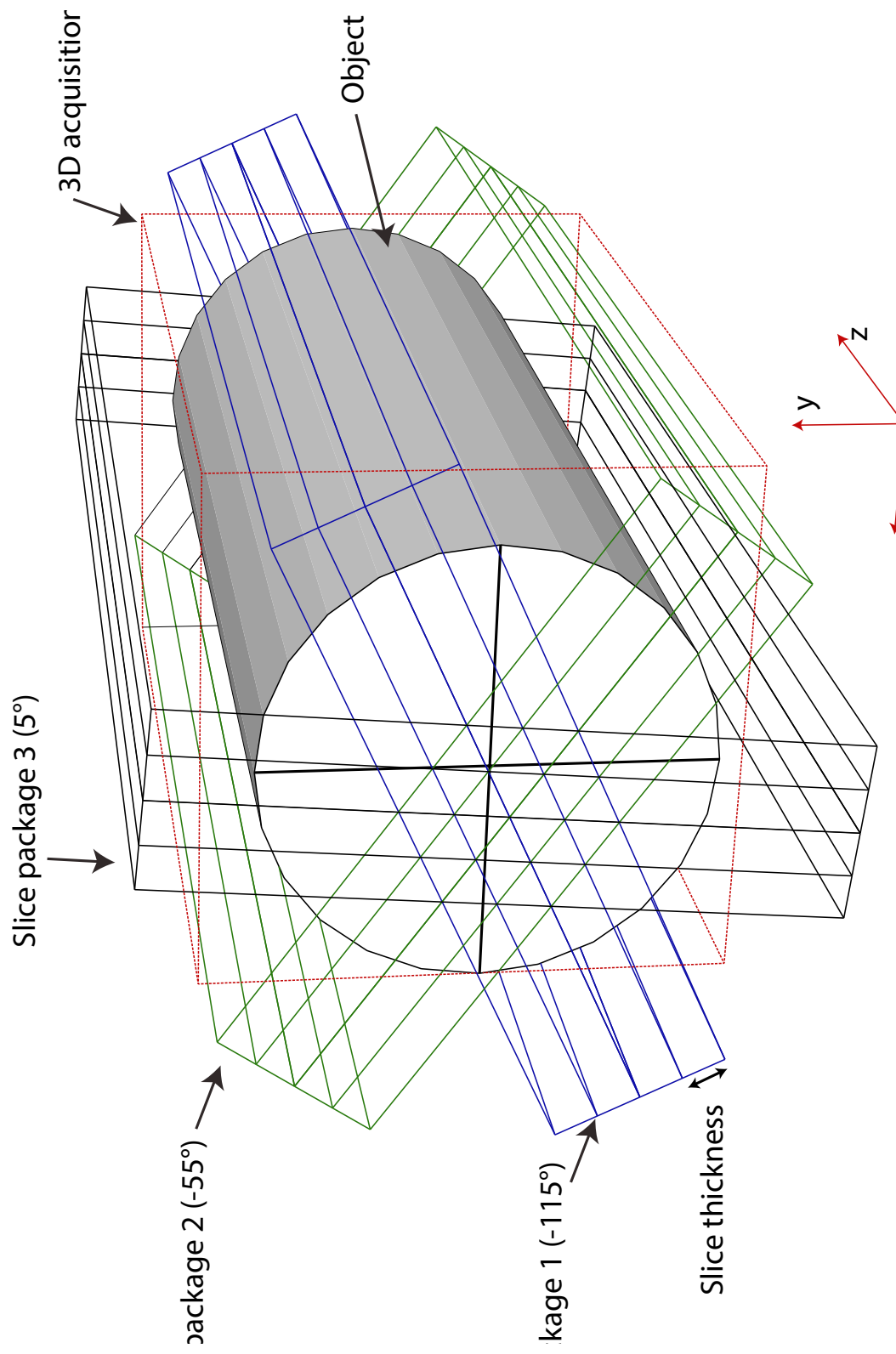

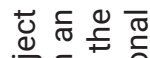
응 훈 은 은

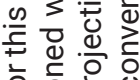
후응음 는도 这 응. 뚱 d 0 N 요욤 는원웅 융 은

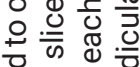
बㄴㄷㄷำ 言 $\underset{0}{0}$ 인 ㄷㅇㅇ 으 흔 넝 등 어는 뜬 으

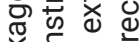
능 인 तั ฯ 으 등 잉 잉 ज 은 토 응 띠 ฮั है 品它

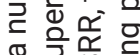

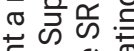

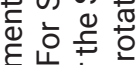
힌믄 믄 里苛

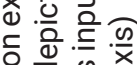
든 응 $\frac{1}{0}$ 인 는 운 ल 등 ฮั

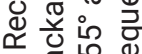
든 을 욜 긍 음 둔 ब ल \० ฯ

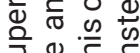
๘

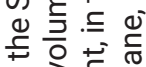
잉 등 나 으 틀

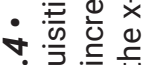

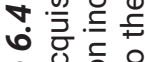
언 은 운 รั่

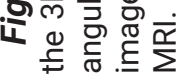


images, the coil was tuned to the ${ }^{19} \mathrm{~F}$ frequency (282 MHz). 2D and 3D (FSE) images were acquired using the scan parameters as listed in Table 6.1. Afterwards, the 2D datasets were postprocessed with the SRR algorithms. For visualization, the resulting image datasets and the 3D acquisition were presented as Maximum Intensity Projections (MIP).

\section{Image analysis of simulations}

The SRR images were compared by a quantitative analysis of the image error, spatial resolution, SNR and low-contrast resolution as a function of the input image (datasets with SNR 2 .. 32 obtained at 3, 5 and 9 angles). Due to the inherently low SNR for fluorine MR imaging, the low-contrast resolution was measured as a function of the acquisition time (i.e. increasing SNR). The resulting graphs represent the performance of the reconstruction techniques in terms of SNR as a function of acquisition time.

\section{Image quality measures}

Image error was measured to assess the reconstruction error over the circular section $A$ in the image by calculating the Root Mean Square Error (RMSE). The error was defined as the pixel-by-pixel difference of the reference HR image (Figure 6.2) and the reconstructed image of the phantom.

Spatial resolution was measured in section B by measuring the FWHM of the line profile. Therefore a line of 20 pixels was selected perpendicular and crossing the line profile. To obtain a high accuracy for the FWHM measurement, an average value was calculated over 50 adjacent lines in the center of section B line.

SNR was measured in section $\mathrm{C}$ by calculating the mean of the signal in the bright circle, 100\% signal intensity, and dividing this by the standard deviation of the background, i.e. the dark circle with $0 \%$ signal intensity.

Low-contrast resolution was measured in section D, where for each input SNR level (2 .. 32) the SNR of the bars was calculated in the reconstructed images. For each bar this results in a series of SNR values that depend on the input SNR. For the low-contrast resolution the detection threshold is defined as the input SNR in the LR images for which an SNR of 5 was obtained in the reconstructed images. This threshold was based on the Rose criterion, which states that an SNR of at least 5 is needed to be able to distinguish image features reliably ${ }^{[24-26]}$. For each bar, the corresponding input SNR to reach this threshold was obtained by interpolation of the calculated SNRS. Subsequently, the corresponding input SNRs that are minimally required to fulfill the Rose criterion were plotted as a function of bar width. This analysis is performed for the $10 \%$ and $30 \%$ contrast bars.

\section{Postmortem experiment}

For the postmortem imaging experiment, the SNR of one jugular vein was measured. A region of interest (ROI) was defined in the vessel and the background, at more than 3 pixels away from the vessel. The noise levels measured in acquired MR images 
Chapter 6

were corrected with the Rayleigh factor [27], due to their non-Gaussian pixel intensity distribution. SNR values were calculated for the reconstructed images of three SRR algorithms and for the 3D MR angiography acquisition. SNR values were presented as function of acquisition time. 


\subsection{Results}

\section{Simulation}

The results of the image error, spatial resolution and SNR are depicted in Figure 6.5 as a function of input SNR for the three algorithms.

Image error. The RMSE for the reconstructed INT and RIBP images is much lower than for the IBP images. At low input SNR, INT has a somewhat lower RMSE, while for an input SNR $\geq 8$ the RMSE of INT and RIBP is comparable.

Spatial resolution. IBP shows the best spatial resolution with a FWHM of 1.0-1.1 pixels for 3 reconstruction angles and an input $S N R \geq 4$. For input $S N R \geq 4$, the FWHM of the RIBP images are 1.1 -1.3 pixels. The INT reconstruction results in the lowest spatial resolution and the FWHM increases with the number of angular positions.

SNR. For all SRR methods the output SNR approximately increases linearly with increasing input SNR. For the INT method, the output SNR increases with the number of angulations, while for the two iterative methods a slight decrease can be observed. Best output SNR is obtained for the INT algorithm, followed by the RIBP.

\section{Low-contrast resolution}

The low-contrast resolution, in terms of the minimal input SNR that is required to observe a bar with a CNR of 5, is shown in Figure 6.6 for a contrast level of $30 \%$ and as a function of bar width. For IBP a stable minimal input SNR for large vessels ( $\geq 3$ pixels diameter) of 25,32 and 35 was found for 3,5 and 9 angulations, respectively. Unexpectedly, this limit was lower for small vessels ( $<3$ pixels). RIBP shows detection limit plateaus at an SNR of 7 for 3 angulations and an input SNR of 12 for 5 and 9 angulations. INT showed the lowest minimal input SNR of 5 .

At a contrast level of $10 \%$ the input SNR required to reach a CNR of 5 was higher for all SRR methods (Figure 6.7). For IBP, the minimally required input SNR to obtain a CNR higher than 5 was above 50. RIBP shows a detection limit at an input SNR of approximately 20 for 3 angulations and approximately 35 for 5 and 9 angulations. INT again showed the lowest minimally required input SNR of approximately 10, except in very small bars (1 pixel) at 3 angulations.

In Figure 6.8 the reconstructed bars are depicted. These images show that at an input SNR of 8 and a contrast level of $30 \%$ all bars can be distinguished from the background. Even with an input SNR of 2, most of the broader bars can to some extent be distinguished from the background for RIBP and INT, but not for IBP. However, the edges of the bars are most sharply depicted for the IBP, which can be observed best for the $30 \%$ contrast bars with an input SNR of 8. The CNRs and background noise levels (standard deviation: STD) are given in Table 6.3.. For IBP all CNR values are below 1.7, although the bars with $30 \%$ contrast can still be seen in the images. Background noise increases with the number of angulations in IBP and RIBP, while for INT a decrease is observed. For 3 angulations the background noise level is of the same order for RIBP and INT, while for IBP the noise level is much higher. 
Chapter 6
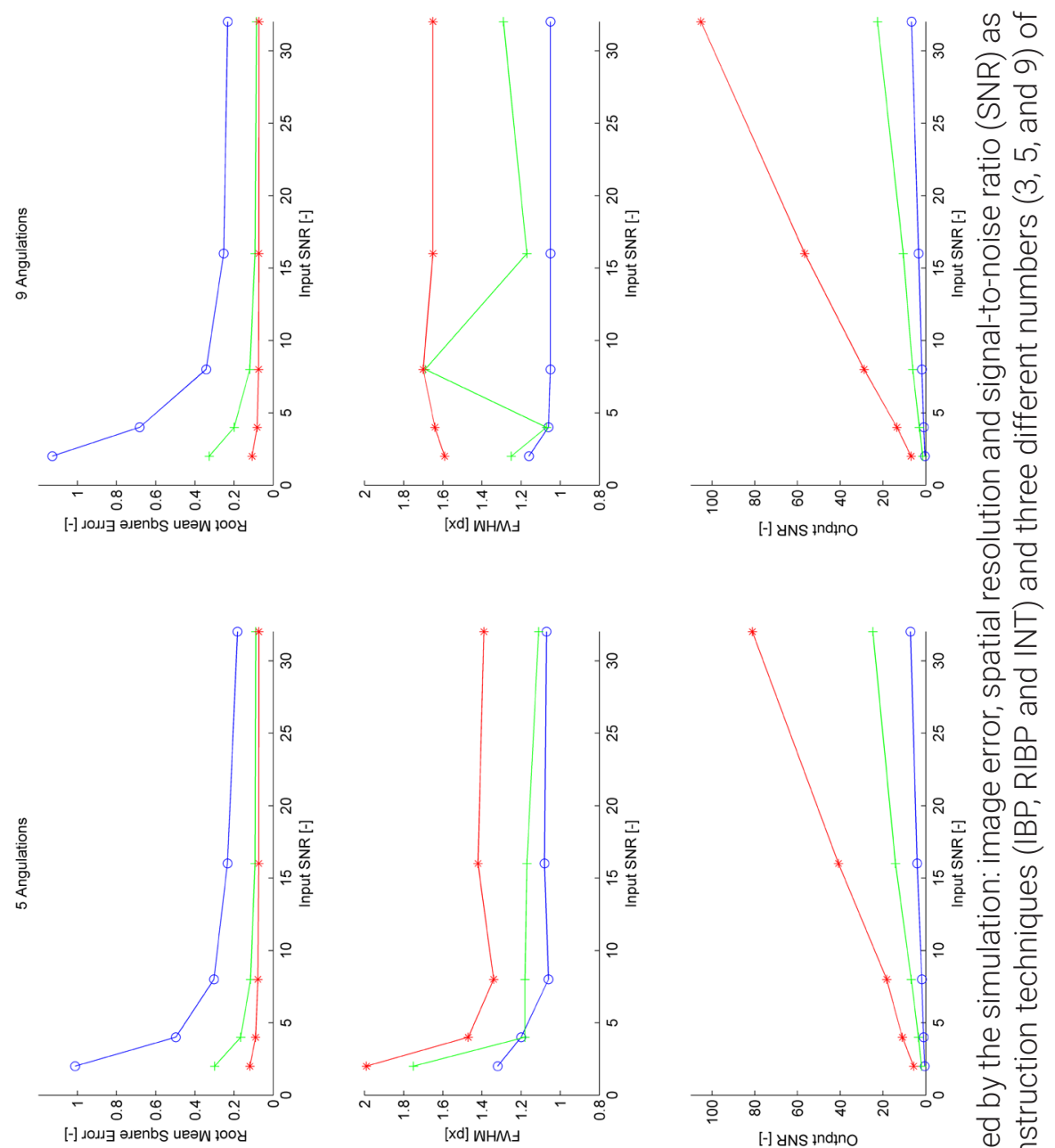

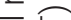

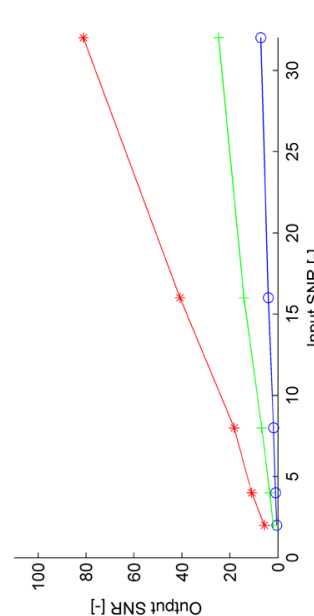

믈

के

$\div$ त

은 음

(4) $\bar{x}$ $\underline{=}$ 言 苧 है (4) 드 궁 응 등 엉
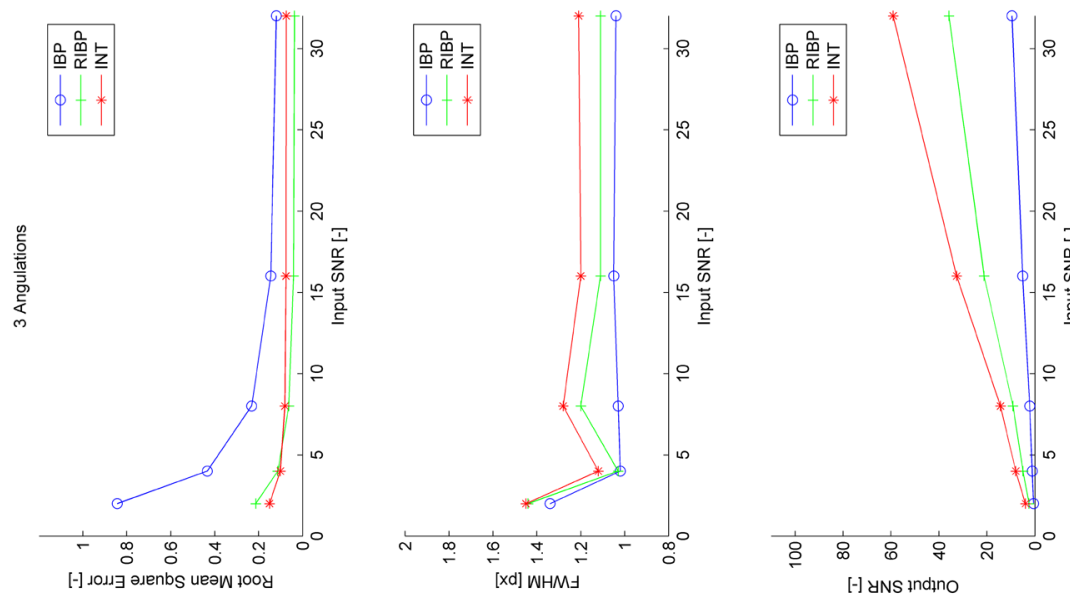
(1) ญ क ष () E 쁜 实

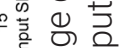
त 트 뜨

(م) ○ั ธิ 约艺艺 운 

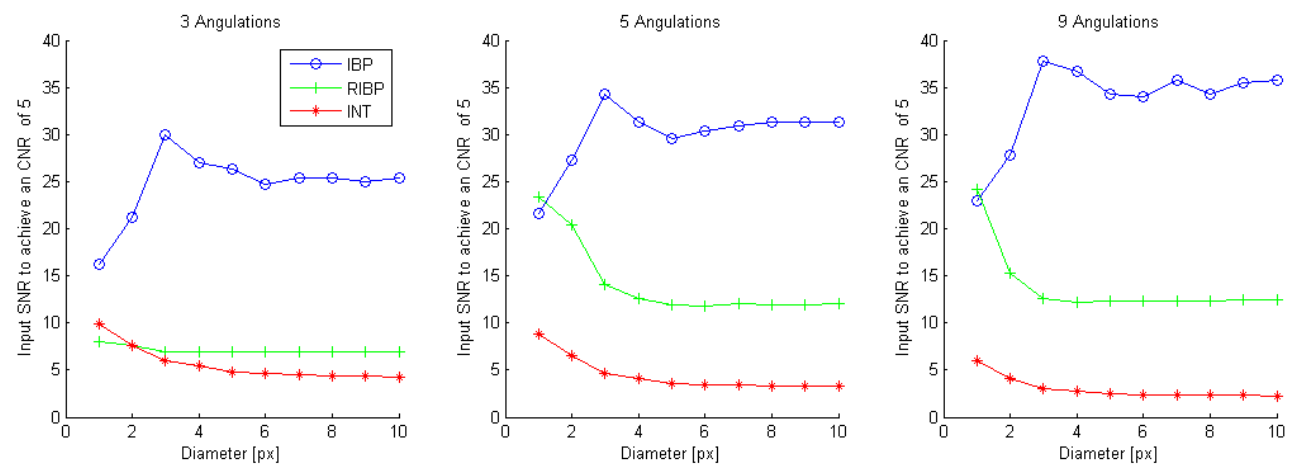

Figure 6.6 - The required input SNR as a function of bar width (mimcking a blood vessel) at a contrast of $30 \%$. For IBP a lower input SNR is required for narrow low-contrast bars with a width of 1-2 pixels, while the minimal input SNR of bars with 3 pixels and wider maintain at a stable SNR level. RIBP and INT show that higher input SNR levels are required for thin bars. For IBP and RIBP a higher input SNR is required for reconstructions of more angulations, whereas for INT a lower input SNR is needed.
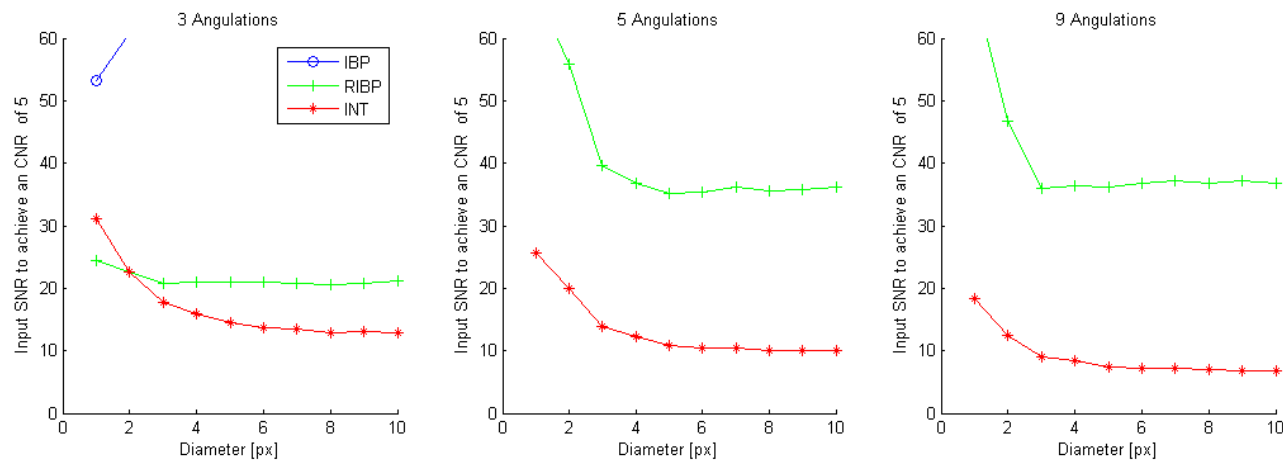

Figure 6.7 - The required input SNR as a function of bar width (mimcking a blood vessel) at a contrast of $10 \%$. In this measurement IBP and RIBP show the same behavior as for the $30 \%$ contrast. For IBP the detection limit is higher than 50 , which did not allow the calculation of an SNR level at this contrast level. 


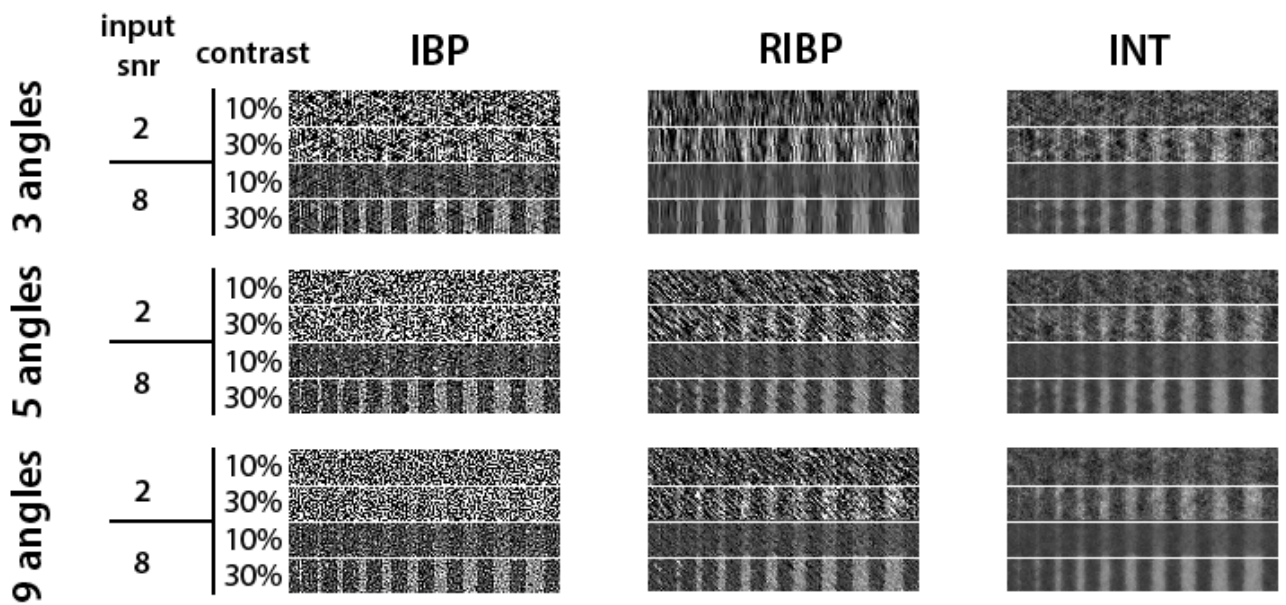

Figure 6.8 Reconstructed bars representing blood vessels. In the images the bars with an original contrast of $10 \%$ and $30 \%$ are depicted. From the series of reconstructed images, only the images with an input SNR of 2 and 8 are shown, for each technique based on source LR images with 3, 5 and 9 angulations, respectively. For IBP with an input SNR of 2 the reconstructed bars cannot be distinguished from the noise. With an input SNR of 8 the bars become visible at both contrast levels, although the thinnest bars with a contrast of $10 \%$ are quite difficult to detect. The edges of the bars in the IBP images are depicted sharply at a SNR level of 8 , which is most clearly observed at $30 \%$ contrast. For RIBP, the bars are already visible with an input SNR of 2 and a contrast of $30 \%$, while the bars with a low contrast of $10 \%$ are only visible at an input SNR of 8. With RIBP, the bar edges are less sharp compared to IBP. For INT, the bars are visible in all cases except for the $10 \%$ contrast level bars with an input SNR of 2 , that have been reconstructed based on 3 and 5 angulations. INT shows the most blurry bar edges.

Table 6.3 - CNR values and noise levels for the bars with a line-width of 10 shown in Figure 6.8. The presented CNR values are resulting from reconstructions using input images with SNR of 8 .

\begin{tabular}{|c|c|c|c|c|c|c|c|c|}
\hline \multirow[b]{2}{*}{$\begin{array}{l}\text { Number of } \\
\text { angulations }\end{array}$} & \multirow{2}{*}{\multicolumn{2}{|c|}{$\begin{array}{l}\text { Contrast } \\
\text { level [\%] }\end{array}$}} & \multicolumn{2}{|c|}{ IBP } & \multicolumn{2}{|c|}{ RIBP } & \multicolumn{2}{|c|}{ INT } \\
\hline & & & CNR & STD & CNR & STD & CNR & STD \\
\hline & \multirow{2}{*}{3} & 10 & 0.35 & 0.17 & 1.76 & 0.04 & 3.12 & 0.03 \\
\hline & & 30 & 1.61 & & 6.45 & & 9.74 & \\
\hline & \multirow{2}{*}{5} & 10 & 0.69 & 0.24 & 1.46 & 0.08 & 4.58 & 0.03 \\
\hline & & 30 & 0.94 & & 3.62 & & 12.82 & \\
\hline & \multirow{2}{*}{9} & 10 & 0.14 & 0.27 & 1.00 & 0.08 & 5.13 & 0.02 \\
\hline & & 30 & 1.16 & & 3.42 & & 17.30 & \\
\hline
\end{tabular}




\section{Postmortem fluorine MR angiography}

The 3D MR fluorine angiograms are depicted in Figure 6.9. The images clearly show that the SNR in the MIP increases as a function of TR, and thus acquisition time. The smallest vessels can be observed best in the image with the longest TR, and thus the longest acquisition time.

MIPs were also produced with the three SRR techniques (Figure 6.10). For IBP, it can be observed that the SNR decreases with an increasing number of angulations (3, 5 , or 9). For the reconstruction with 3 angles, the largest NSA and thus the longest acquisition time has the highest SNR. RIBP also does not strongly benefit from more angulations in terms of noise reduction, although the visualization of small vessels is slightly improved with an increasing number of angulations. The INT images reconstructed with a higher number of angulations are less noisy and small vessels become more clearly resolved. The visual detail level reaches a maximum at an acquisition time of 118 minutes for INT, after which no further visual improvement in detail level is observed.

To show the effect of SRR at higher detail, a region of interest was chosen in the jugular vein (Figure 6.11.). For each method, the images with the longest acquisition time are presented, which was 237 minutes for the SRR and 409 minutes for the 3D acquisition. In this series of images, the IBP reconstruction suffers most from noise, resulting in low contrast of the vein. RIBP has a relatively high noise level, while the image contrast is better than for IBP. INT has a very low noise level, facilitating a clear depiction of the vessel, although the vessel edges are blurred due to the relatively low spatial resolution. The native 3D acquisition shows the best contrast and spatial resolution; note however that the acquisition time was twice as long as for the SRR methods. For all datasets, the SNR was measured for 3, 5 and 9 angulations and for all the TR settings of the native 3D dataset as a function of acquisition time (Figure 6.12.). For each dataset, INT has the highest SNR, even higher than for the 3D acquisition. RIBP shows nearly comparable results to the 3D acquisition, except for the images obtained for 9 angulations, where 3D acquisition outperforms RIBP. For all measurements, IBP gives the lowest SNR.

For acquisition times less than 40 min, RIBP and INT have better image contrast (in the thorax and heart region), more image details (clearer depiction of the smaller vessels), and lower noise levels than 3D acquisition (Figure 6.9 and Figure 6.10.). For acquisition times above 80 minutes however, the 3D acquisitions perform better than the SRR methods and reveal more detail. 

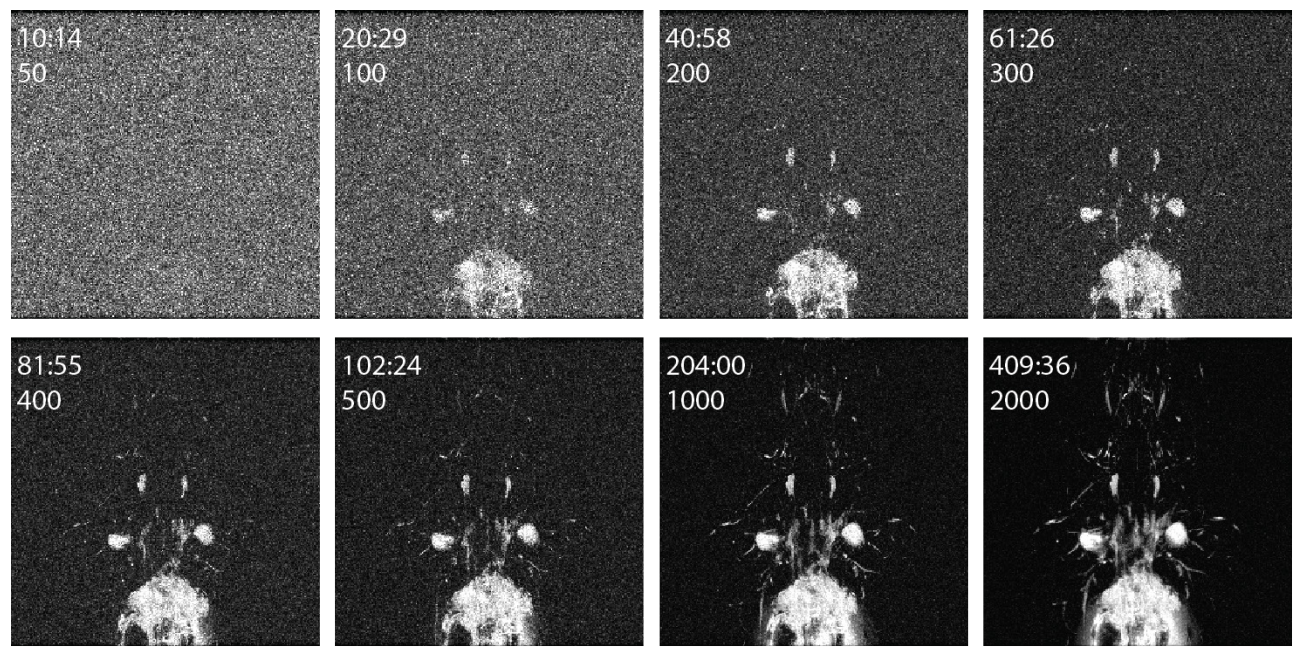

Figure 6.9 - Coronal maximum intensity projections of the native 3D MRI. The MIP is projected on the coronal plane. The thorax and heart region is located at the bottom of each image, and the main brain vessels in the center of the images. In the upper left corner of each image is the acquisition time (notation, min:sec) and the repetition time (TR in ms) are depicted. For an increasing acquisition time (and TR), the SNR increases and more details can be observed of the vasculature.

Figure 6.10 - (page 119) Coronal maximum intensity projections of the SRR images based on various angulations $(3,5$, ad 9$)$ and low-resolution input images with varying $\operatorname{NSA}(1,2,4$, 8 and 16). In the upper left corner of each image the acquisition time is depicted (min:sec). For IBP, it can be observed that the SNR decreases with an increasing number of angulations. The reconstruction using input images at 3 different angles and NSA of 16, and therefore the lowest acquisition time, has the highest SNR. RIBP does not highly benefit from more angulations in terms of noise reduction, although the visualization of small vessels is slightly improved with an increasing number of angulations. These reconstructions also show some artifacts in the form of horizontal bands with noise, but the artefact is not present in every reconstruction. The INT images reconstructed using more angulations are less noisy and small vessels can be more clearly resolved. 


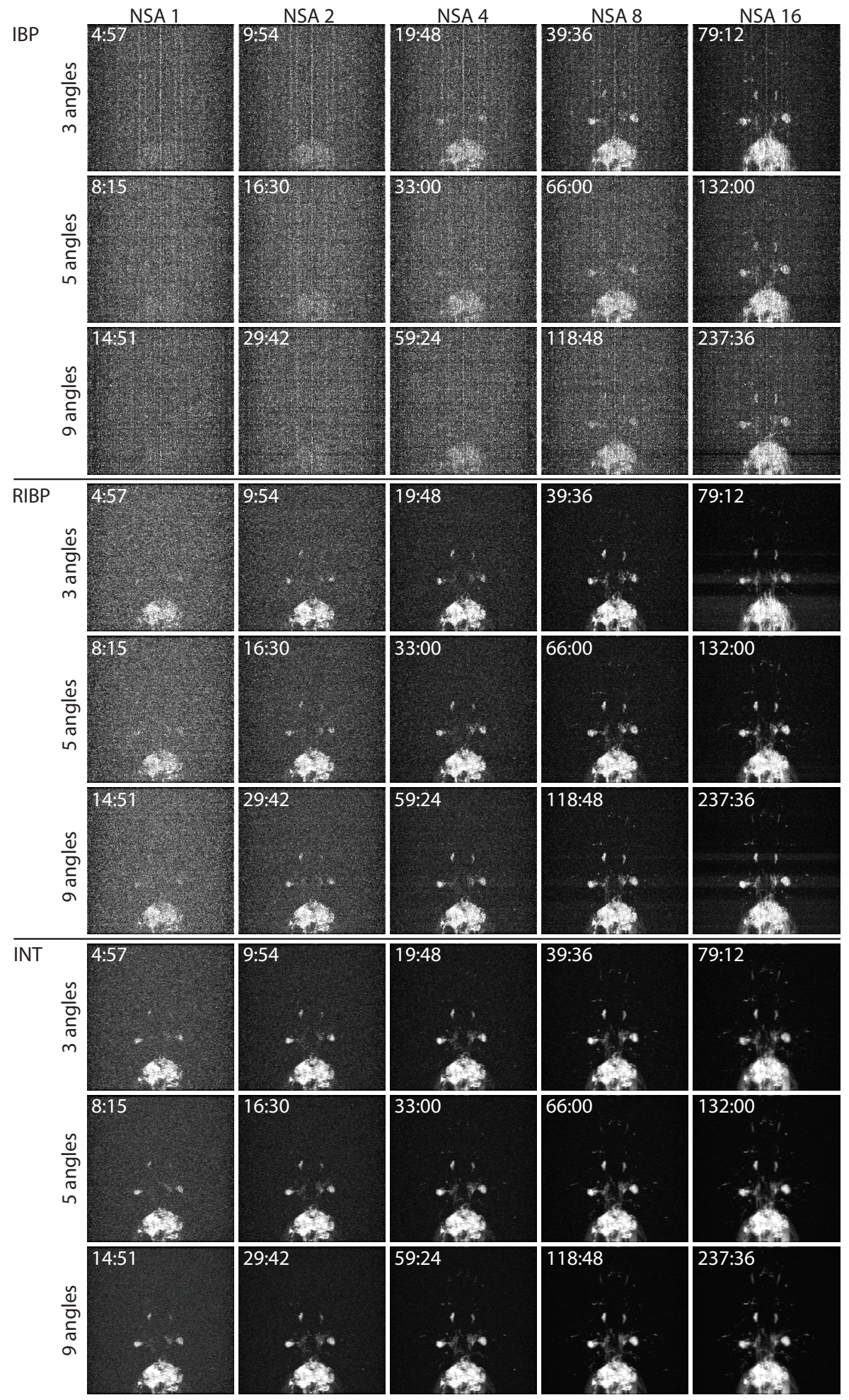


IBP
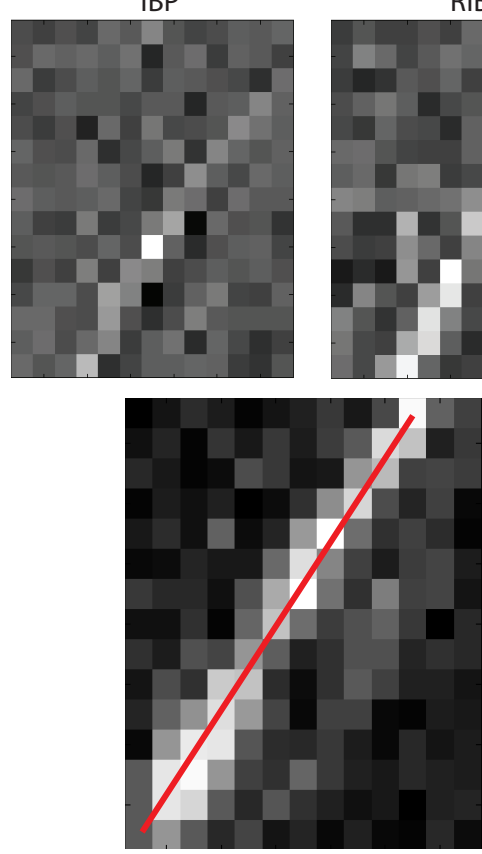

Vessel selection

\begin{abstract}
.
\end{abstract}

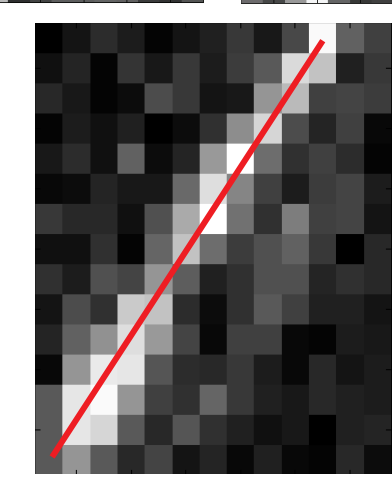

RIBP

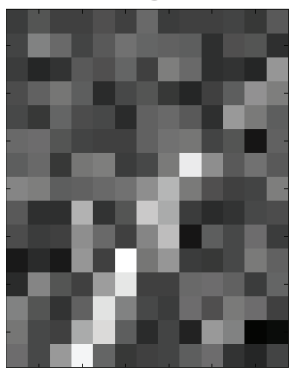

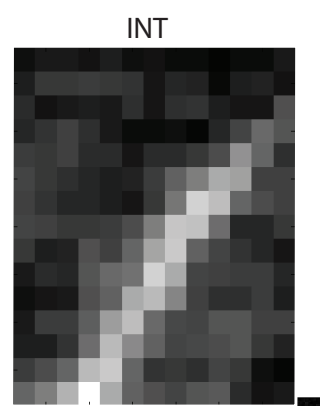

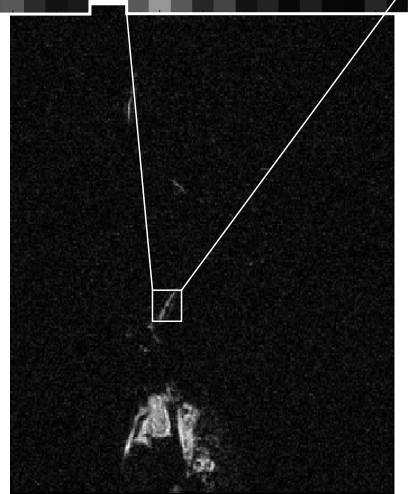

Single slice of the native 3D acquisition

Figure 6.11 - Fluorine MR angiograms of the jugular vein in a mouse model. Depicted are the three super-resolution reconstructions ( $\mathrm{a}$, IBP; $\mathrm{b}$ RIBP; and c, INT), the native 3D acquisition (d), and the native 3D image with marked pixels of the vessel (red line), and the full field-ofview fluorine MR image of the thorax-head part with a region-of-interest corresponding to the previous images (a-e). Pixels that are intersected by the red line were marked as blood vessel, the background voxels were selected within a distance of at least 3 pixels from the red line
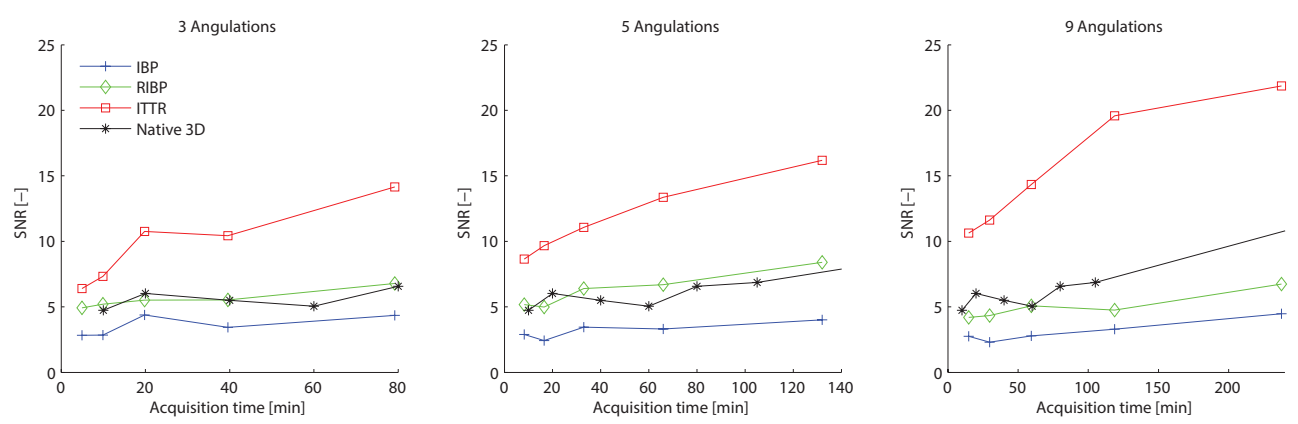

Figure 6.12 - The SNR of the angiograms as function of the acquisition time. The figure shows the results of 3,5 and 9 angulations for the three reconstruction techniques and the native $3 \mathrm{D}$ acquisition. IBP provides the lowest SNR, RIBP shows nearly comparable results with the 3D acquisition, while INT gives the highest SNR. 


\subsection{Discussion}

\section{Main findings}

In high resolution 3D fluorine based MR angiography, the relatively long $T$, of fluorine, results in a very long acquisition time. With the current study, we have shown that the various SRR techniques can reduce the acquisition time by replacing the 3D acquisition with a combination of relatively fast multi-angle multi-slice acquisitions and dedicated reconstruction techniques. Each SRR method appears to have specific advantages, but also drawbacks. IBP provides the best spatial resolution performance, while the INT technique outweighed the other iterative reconstruction techniques in (output) SNR when the input images for SRR have minimal SNR. RIBP could be a good compromise in certain cases, as it demonstrated to provide acceptable lowcontrast resolution and spatial resolution, together with a better (output) SNR than IBP. For postmortem ${ }^{19} \mathrm{~F}$ angiography, the best performance was obtained with the INT technique, since low input SNR was the strongest limitation.

\section{Image error and SNR}

In the quantitative analysis, the image error was comparable for INT and RIBP, and worst for IBP. For IBP, the error further increased with higher number of angulations. This behavior is probably related to SNR transduction in the IBP algorithm, where SNR gain decreases with the number of angulations. Quantitative analysis of the three SRR techniques in the simulation and the postmortem angiograms showed that INT has superior output in terms of SNR. This technique apparently benefits from the multi-voxel averaging processes that are inherent in the interpolation reconstruction kernel. The multi-voxel interpolation results in a strong SNR gain and enhances the noisy input images. The simulations and postmortem angiography show that the IBP images have the lowest SNR. This can be explained by the averaging and subtraction operation in the IBP kernel, which is more prone to noise than the median and subtraction operation of the RIBP kernel.

With an increasing number of input images (i.e. more angulations), one might expect a reduction in image error and a better SNR. This is indeed observed for INT as the interpolation operation reduces the noise (by smoothing). However, for the iterative reconstruction techniques, IBP and RIBP, an increasing image error was found with an increasing number of angulations. This is likely due to the subtraction step in the iterative techniques, which inherently propagates noise and will lower the SNR. Also van Reeth et al ${ }^{[23]}$ found that the SNR does not increase for RIB with an increasing number of LR input images.

\section{Spatial resolution}

The phantom simulation analysis demonstrates the superiority of the iterative techniques, IBP and RIBP, for the reconstructed spatial resolution. However, these 
methods need a minimal input SNR of 8 to avoid reconstructed HR images that are too noisy.

For the reconstruction of the HR image, the angulations of the LR images were chosen so that there were not parallel to the axes of the HR image (i.e. not at an angle of $0^{\circ}$ or 90 ). For INT we observed a decrease in spatial resolution with an increasing number of reconstruction angulations. It is possible that more blurring was introduced by interpolating the voxels of relatively thick slices with more angulations. IBP and RIBP, in contrast, did not show a spatial resolution dependency on the number of angulations. Instead, the subtraction operation in the backprojection kernel may sharpen the reconstructed images.

\section{Low-contrast resolution}

For the phantom simulations, the IBP method demonstrated a high spatial resolution, at the cost of a high noise level. The detection of low contrast objects will therefore be impeded. The RIBP method is more resistant to noise and results in a superior low-contrast resolution compared to IBP. However, the INT kernel reconstructs with the highest SNR levels and with the best low-contrast resolution. The INT method interpolates on the voxels of all angulations, where the iterative methods try to minimize the error in the HR image in a number of iteration steps.

The reconstructed postmortem INT angiogram (Figure 6.11.) has a better contrast detail level compared to the iterative methods. In these angiograms, SNR rather than spatial resolution seems to be the limiting factor in the detection of vessels in lowcontrast conditions.

We have shown in the postmortem experiment that for short acquisition times $(<40$ minutes), RIBP and INT show superior image quality in comparison to the native 3D MR images. The RIBP and INT images showed improved image contrast (thorax and heart), more image details (improved conspicuity of smaller vessels), and better SNR levels. For longer acquisition times (> 80 minutes) the 3D image quality was better than the SRR methods and revealed more detail. Quantitative measurements of the SNR in the postmortem images demonstrate a superior SNR for INT compared to the native 3D MR images at the same acquisition time (Figure 6.12.). The iterative techniques do not improve the SNR compared to the 3D acquisition.

\section{Preclinical application of SRR}

The main advantage of using SRR methods is to overcome the low acquisition efficiency of fluorine contrast media due to its long $T_{1}$ relaxation times. This study shows the efficiency of three SRR techniques with noisy fluorine MR images as input. Until now this efficiency was only assessed for proton MRI. Plenge et al compared different reconstruction methods for proton MRI ${ }^{[21]}$. They also found better spatial resolution performance for IBP compared to RIBP in their image contrast. This is probably due to the lower noise level of their input images of the (Shepp-Logan) phantom mimicking brain anatomy. Their simulations had a noise level of 32dB (SNR of 39.8), while in the 
present study we focused on low-contrast conditions with noise levels of 6.0 and 18.0 $\mathrm{dB}$ (SNR of 2 and 8), respectively, which are typical for fluorine angiography.

The current study shows that with the same dataset multiple reconstructions can be made. This is not only beneficial in the comparison of different techniques, but it can also contribute to other studies to make multiple reconstructions tailored to specific image features, for instance spatial resolution, low contrast resolution or SNR gain.

To obtain high quality MR angiograms, the vessel lumen is preferably visualized with a high spatial resolution and high contrast compared to surrounding tissue. Depending on the needs of a study, SRR techniques can be used under specific conditions. Put differently, different SRR techniques can subsequently be applied to the same input image data set, after which the obtained SRR images can be used to enrich various aspects (sharpness, contrast, etc) of the visualized anatomic structures. Based on our findings for low contrast fluorine imaging, the robust iterative variant RIBP can be used when the LR images have an $S N R>8$. For images acquired with lower $S N R$, the INT method is advised, although this method results in an inferior spatial resolution. 


\subsection{Conclusions}

This study showed the possibilities of three SRR methods, IBP, RIBP and INT for fluorine MR angiography, in conditions with low contrast levels. When acquisition time is no issue, a 3D MRI shows most details in fluorine MR angiography. At shorter acquisition times however, RIBP showed good performance in terms of spatial resolution when sufficient input SNR is provided. INT provides overall the highest gain in SNR, and thus contrast resolution, which is advantageous over native 3D MRI acquisitions by reduction of acquisition time. 


\section{References}

1. Neubauer, A.M., S.D. Caruthers, F.D. Hockett, T. Cyrus, J.D. Robertson, J.S. Allen, T.D. Williams, R.W. Fuhrhop, G.M. Lanza, and S.A. Wickline, Fluorine cardiovascular magnetic resonance angiography in vivo at $1.5 \mathrm{~T}$ with perfluorocarbon nanoparticle contrast agents. J Cardiovasc Magn Reson, 2007. 9(3): p. 565-73.

2. Wolters, M., S.G. Mohades, T.M. Hackeng, M.J. Post, M.E. Kooi, and W.H. Backes, Clinical perspectives of hybrid proton-fluorine magnetic resonance imaging and spectroscopy. Invest Radiol, 2013. 48(5): p. 341-50.

3. van Heeswijk, R.B., Y. Pilloud, U. Flogel, J. Schwitter, and M. Stuber, Fluorine-19 magnetic resonance angiography of the mouse. PLoS One, 2012. 7(7): p. e42236.

4. de Vries, A., R. Moonen, M. Yildirim, S. Langereis, R. Lamerichs, J.A. Pikkemaat, S. Baroni, E. Terreno, K. Nicolay, G.J. Strijkers, and H. Grull, Relaxometric studies of gadoliniumfunctionalized perfluorocarbon nanoparticles for MR imaging. Contrast Media Mol Imaging, 2014. 9(1): p. 83-91.

5. Shilling, R.Z., T.Q. Robbie, T. Bailloeul, K. Mewes, R.M. Mersereau, and M.E. Brummer, A super-resolution framework for 3-D high-resolution and high-contrast imaging using 2-D multislice MRI. IEEE Trans Med Imaging, 2009. 28(5): p. 633-44.

6. Greenspan, H., G. Oz, N. Kiryati, and S. Peled, MRI inter-slice reconstruction using superresolution. Magn Reson Imaging, 2002. 20(5): p. 437-46.

7. Gholipour, A., O. Afacan, I. Aganj, B. Scherrer, S.P. Prabhu, M. Sahin, and S.K. Warfield, Super-resolution reconstruction in frequency, image, and wavelet domains to reduce through-plane partial voluming in MRI. Med Phys, 2015. 42(12): p. 6919-32.

8. Gholipour, A., J.A. Estroff, and S.K. Warfield, Robust super-resolution volume reconstruction from slice acquisitions: application to fetal brain MRI. IEEE Trans Med Imaging, 2010. 29(10): p. 1739-58.

9. Pier, D.B., A. Gholipour, O. Afacan, C. Velasco-Annis, S. Clancy, K. Kapur, J.A. Estroff, and S.K. Warfield, 3D Super-Resolution Motion-Corrected MRI: Validation of Fetal Posterior Fossa Measurements. J Neuroimaging, 2016. 26(5): p. 539-44.

10. Dzyubachyk, O., Q. Tao, D.H. Poot, H.J. Lamb, K. Zeppenfeld, B.P. Lelieveldt, and R.J. van der Geest, Super-resolution reconstruction of late gadolinium-enhanced MRI for improved myocardial scar assessment. J Magn Reson Imaging, 2015. 42(1): p. 160-7.

11. Poot, D.H., B. Jeurissen, Y. Bastiaensen, J. Veraart, W. Van Hecke, P.M. Parizel, and J. Sijbers, Super-resolution for multislice diffusion tensor imaging. Magn Reson Med, 2013. 69(1): p. 103-13.

12. Poot, D.H., V. Van Meir, and J. Sijbers, General and efficient super-resolution method for multi-slice MRI. Med Image Comput Comput Assist Interv, 2010. 13(Pt 1): p. 615-22.

13. Scherrer, B., A. Gholipour, and S.K. Warfield, Super-resolution reconstruction to increase the spatial resolution of diffusion weighted images from orthogonal anisotropic acquisitions. Med Image Anal, 2012. 16(7): p. 1465-76.

14. Ning, L., K. Setsompop, O. Michailovich, N. Makris, M.E. Shenton, C.F. Westin, and Y. Rathi, A joint compressed-sensing and super-resolution approach for very high-resolution diffusion imaging. Neuroimage, 2016. 125: p. 386-400. 
15. Ning, L., K. Setsompop, O. Michailovich, N. Makris, C.F. Westin, and Y. Rathi, A CompressedSensing Approach for Super-Resolution Reconstruction of Diffusion MRI. Inf Process Med Imaging, 2015. 24: p. 57-68.

16. Van Steenkiste, G., D.H. Poot, B. Jeurissen, A.J. den Dekker, F. Vanhevel, P.M. Parizel, and J. Sijbers, Super-resolution $T_{1}$ estimation: Quantitative high resolution $T_{1}$ mapping from a set of low resolution $T_{1}$-weighted images with different slice orientations. Magn Reson Med, 2016.

17. Irani, M. and S. Peleg, Improving Resolution by Image Registration. Cvgip-Graphical Models and Image Processing, 1991. 53(3): p. 231-239.

18. Irani, M. and S. Peleg, Motion Analysis for Image Enhancement: Resolution, Occlusion, and Transparency. Journal of Visual Communication and Image Representation, 1993. 4(12): p. 324-335.

19. Zomet, A., A. Rav-Acha, and S. Peleg, Robust super-resolution. 2001 leee Computer Society Conference on Computer Vision and Pattern Recognition, Vol 1, Proceedings, 2001: p. 645650.

20. Vandewalle, P., S. Susstrunk, and M. Vetterli, A Frequency Domain Approach to Registration of Aliased Images with Application to Super-resolution. EURASIP Journal on Applied Signal Processing, 2006. 2006: p. 1-14.

21. Plenge, E., D.H. Poot, M. Bernsen, G. Kotek, G. Houston, P. Wielopolski, L. van der Weerd, W.J. Niessen, and E. Meijering, Super-resolution methods in MRI: can they improve the trade-off between resolution, signal-to-noise ratio, and acquisition time? Magn Reson Med, 2012. 68(6): p. 1983-93.

22. Mahmoudzadeh, A.P. and N.H. Kashou, Interpolation-based super-resolution reconstruction: effects of slice thickness. J Med Imaging (Bellingham), 2014. 1(3): p. 034007.

23. Van Reeth, E., C.H. Tan, I.W. Tham, and C.L. Poh, Isotropic reconstruction of a 4-D MRI thoracic sequence using super-resolution. Magn Reson Med, 2015. 73(2): p. 784-93.

24. Oostendorp, M., M.J. Post, and W.H. Backes, Vessel growth and function: depiction with contrast-enhanced MR imaging. Radiology, 2009. 251(2): p. 317-35.

25. Burgess, A.E., The Rose model, revisited. J Opt Soc Am A Opt Image Sci Vis, 1999. 16(3): p. 633-46.

26. Rose, A., Vision - Human and Electronic. 1973: Plenum Press. .

27. Haacke, E.M., R.W. Brown, M.R. Thompson, and R. Venkatesan, Magnetic resonance imaging : physical principles and sequence design. 1999, New York: Wiley. xxvii, 914 p. 


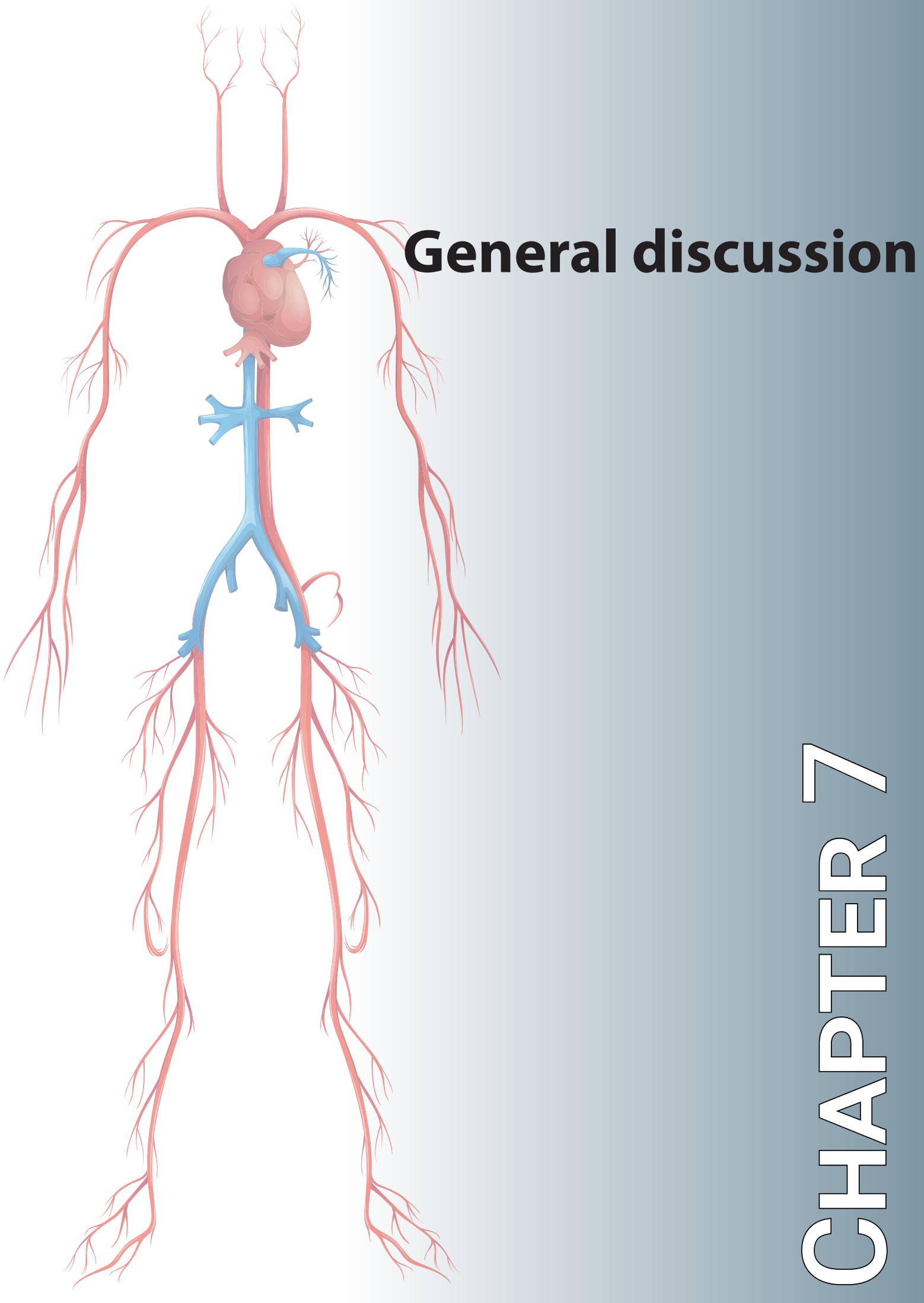


Chapter 7 


\subsection{Contrast media for vascular MRI}

For vascular MR imaging it is important to obtain optimal contrast between the vascular lumen and surrounding tissue (MR angiography) or to highlight molecular targets, usually at the endothelium of the vessel wall, which represent specific pathophysiologic features (molecular MRI). This can be achieved by administration of an MRI contrast medium. The use of gadolinium based contrast agents (GBCAs) is most common due to its strong contrast enhancement and the low risk of side effects. Despite the wide clinical use of GBCAs, other MR contrast media (e.g. based on iron-oxides particles or ${ }^{19}$ fluorine) could have specific advantages for vascular MRI. In Table 7.1 the most important properties of GBCAs, iron oxides and ${ }^{19} \mathrm{~F}$ nanoparticles for vascular imaging are listed. The main goal of this thesis is to assess the applicability of iron oxides and ${ }^{19} \mathrm{~F}$ based contrast agents and their associated imaging techniques in preclinical MR studies. This thesis addresses four important aspects of MR contrast media in preclinical vascular MRI applications: suppression of background signal, detectability, linearity, and multi-spectral qualities.

Table 7.1 - MR applicability of gadolinium, iron-oxides and fluorine-based MRI contrast media

\begin{tabular}{|c|c|c|c|c|c|c|}
\hline & $\begin{array}{c}\text { Detectable } \\
\text { nucleus }\end{array}$ & $\begin{array}{l}\text { Detection } \\
\text { mechanism }\end{array}$ & Background Enhancement & $\begin{array}{l}\text { Detection } \\
\text { limit* }^{\star} \\
(\mathrm{mol} / \mathrm{l})\end{array}$ & $\begin{array}{c}\text { Linearity } \\
\text { of signal } \\
\text { intensity vs } \\
\text { concentration }\end{array}$ & Artifacts \\
\hline Gadolinium & ${ }^{7} \mathrm{H}$ & $\begin{array}{l}T_{1} \\
\text { relaxation } \\
\text { of water } \\
\text { protons }\end{array}$ & $\begin{array}{cc}\text { Soft-tissue } & \text { Hyperintense } \\
\text { anatomy } & \text { on } T_{1} \text { w image }\end{array}$ & $1 \cdot 10^{-5}[1,2]$ & $\begin{array}{l}\text { Linear for low } \\
\text { concentrations }\end{array}$ & $\begin{array}{l}\text { Susceptibility } \\
\text { at high } \\
\text { concentrations } \\
\left(T_{2}, T_{2}^{*}\right)\end{array}$ \\
\hline Iron-oxide & ${ }^{1} \mathrm{H}$ & $\begin{array}{l}T_{2} \\
\text { relaxation } \\
\text { of water } \\
\text { protons }\end{array}$ & $\begin{array}{cc}\text { Soft-tissue } & \text { Hypointense } \\
\text { anatomy on } T_{2} \text { w image }\end{array}$ & $1 \cdot 10^{-14}[3]$ & Non-linear & $\begin{array}{c}\text { Signal voids } \\
\text { and blooming } \\
\qquad\left(T_{2}^{*}\right)\end{array}$ \\
\hline${ }^{19}$ Fluorine & ${ }^{19} \mathrm{~F}$ & $\begin{array}{l}\text { Fluorine } \\
\text { spin density }\end{array}$ & $\begin{array}{cc}\text { No } & \text { Hyperintense } \\
\text { detectable } & \text { on spin } \\
\text { background } & \text { density image } \\
\text { signal }\end{array}$ & $4 \cdot 10^{-1}[4]$ & Linear & Chemical shift \\
\hline
\end{tabular}

*Detection limit of each contrast media used as encapsulated nanoparticles. 


\subsection{Background signal}

\section{Positive contrast techniques for iron oxides}

Nanoparticles containing iron oxides induce local magnetic field inhomogeneities (field gradients) that lead to $T_{2}{ }^{*}$ weighted susceptibility changes even at low concentrations. Because this is a strong effect, it was demonstrated that already the presence of a single iron oxides particle can be detected with $\mathrm{MRI}^{[5,6]}$. This high sensitivity makes iron oxides attractive for molecular imaging as molecular targets are usually expressed in very low concentrations.

Comparable to GBCAs, iron oxides havean indirect contrast effect. The contrast medium itself is not directly detected, but instead, it affects the relaxation of the proton signal. The susceptibility effects induce a negative contrast with the surrounding tissue as the signal intensity in the proximity of iron oxides particles is hypointense on MR images. Signal hypointensities are difficult to distinguish from the variations in the background signal of the underlying anatomy, especially near air cavities or bone structures, which already have low signal intensity. In medical imaging, positive contrast is therefore preferred. For this purpose, several positive contrast techniques for the visualization of iron oxides nanoparticles as a hyperintense signal have been developed ${ }^{[7-13]}$. In Chapter 2, White Marker and Susceptibility Gradient Mapping (SGM) were compared as positive contrast imaging methods. These methods make use of the magnetic field gradient effect of the iron-oxides particles. This effect induces a decrease in signal intensity on magnitude images and affects the phase of the local magnetization, which is registered in the phase MR images. The SGM post-processing method makes use of both the original magnitude and phase information. We showed that it was more suitable than White Marker pulse sequences to transform the susceptibility changes into a positive contrast effect, possibly due to restricted sensitization for generation of positive contrast only in the slice selection direction of the White Marker pulse sequence. The extent of contrast enhancement of SGM (positive contrast) was comparable in magnitude with conventional Gradient Echo acquisitions (negative contrast), but opposite in sign, and therefore did not compromise the image quality of the anatomical image. Positive contrast enhancement methods that are based on post-processing of the conventional diagnostic anatomic scans, e.g. SGM, do not increase the scan time with additional acquisitions. This is a strong advantage of post-processing-based positive contrast techniques over dedicated pulse sequencesbased methods, such as White Marker, for clinical translation.

\section{Susceptibility artifacts}

Iron oxides contrast media are usually depicted using $T_{2}{ }^{*}$ weighted gradient echo MRI pulse sequences. A disadvantage of these sequences is that gradient echo imaging techniques are also sensitive to local magnetic field inhomogeneities not related to the iron oxides particles (e.g. tissue-air boundaries). In this respect, also the magnetic effects of excipients or biomaterials used in animal models must be considered. For instance, the use of iron trichloride $\left(\mathrm{FeCl}_{3}\right)$ for the induction of an artificial thrombus 
is well-established in animal models of thrombosis. However, already very low concentrations of $\mathrm{FeCl}_{3}$ can compromise the MRI visualization by causing both hypoand hyperintensities (image artifacts) in the vicinity of the thrombus as shown in Chapter 3. In this chapter, we show that the residuals of $\mathrm{FeCl}_{3}$ can induce significant artifacts, whereas for aluminum trichloride $\left(\mathrm{AlCl}_{3}\right)$ this effect is much less. Furthermore, this study shows that inducing thrombi with $\mathrm{AlCl}_{3}$ results in a comparable thrombus composition as with $\mathrm{FeCl}_{3}$ (see appendix A of Chapter 3). Therefore, to avoid such strong image artifacts by magnetic field inhomogeneities of iron, we successfully applied the induction of thrombi with $\mathrm{AlCl}_{3}$ in Chapter 5. Hereafter, the method of inducing thrombi with $\mathrm{AlCl}_{3}$ was also adopted by other investigators ${ }^{[14,15]}$. In Chapter 5 we used $a^{19} \mathrm{~F}$ contrast medium, which is not hampered by any background signal when measured on the fluorine resonance frequency. It is important to note that also for other resonance frequencies (e.g. ${ }^{19} \mathrm{~F}$ ) than that of ${ }^{1} \mathrm{H}$ it remains relevant to avoid magnetic field inhomogeneities (e.g. due to iron particles) as much as possible as it affects the excitability of the nuclear spins and thus also the detectability of (e.g. fluorine based) contrast media with a positive contrast effect. 


\subsection{Detectability}

The signal intensity changes generated by a contrast medium depend on the field strength of the MR system and the local (in vivo or in vitro) contrast agent concentration. To be detectable, the signal intensity must be higher than a specific threshold, typically the background signal variation or noise level. Chapter 1 already describes that the relation between signal intensity and concentration, in general, is non-linear for indirect contrast media. The relaxivity of GBCAs decreases with increasing field strength and therefore the detectability decreases. For iron oxides and fluorine, on the contrary, the detection limit for the lowest concentration improves with increasing field strength. Especially for pre-clinical research, which is usually performed at high field strengths ( $>6$ Tesla), this relationship is beneficial for iron oxides and fluorine based contrast media.

Although a higher field strength is beneficial for fluorine imaging, other challenges still remain. In fluorine imaging, the administered fluorine density is detected directly on the basis of its (nuclear) spin density. However, for fluorine MR imaging the spin density is at least a factor 10 smaller compared to $\left({ }^{1} \mathrm{H}\right.$ in) water molecules of human tissue, because the (maximum) amount of fluorine that can be administrated limits its local concentration. Besides this limited achievable concentration, the signal yield is approximately $80 \%$ relative to that of (water) protons. These are factors that make fluorine imaging more challenging than GBCAs or iron oxides based contrast media. For angiography, the high concentrations that are required for fluorine MR can be achieved in the lumen of the blood vessels when the contrast is administered through a direct intravascular route.

Fluorine has a relatively long $T$, relaxation time (>1000 ms below 3 Tesla), which is the most important factor that determines the acquisition time ${ }^{[16]}$. The acquisition time depends on the repetition time of an MR pulse sequence. A short repetition time will shorten the scan protocol, but will counteract the recovery of the longitudinal magnetization and in this way saturates the MR signal. Several strategies have been developed to overcome the long acquisition times typically needed for fluorine imaging. For instance, De Vries et al. showed that the longitudinal relaxation time of fluorine can be shortened by adding functionalized Gd(III)DTPA complexes [16]. Another method to shorten the acquisition time for fluorine imaging, in particular angiography, is to use super-resolution Reconstruction (SRR) techniques ${ }^{[17-20]}$. In chapter 6 we compare various SRR techniques and showed that the detection of fluorine contrast media can be enhanced with multiple relatively short multi-slice acquisitions orientated at different angulations, in combination with special postprocessing techniques. We show under conditions of low signal-to-noise levels, as for instance in fluorine angiography, how SRR techniques are beneficial over conventional 3D acquisition techniques. At shorter scan times SSR provides more spatial details and a better signal-to-noise ratio compared to 3D acquisitions. 


\subsection{Linearity}

Quantitative analysis of contrast-enhanced MRI can be beneficial in the assessment of a disease or monitoring during treatment. For this, an estimation of the local contrast medium concentration is desired. To determine this concentration in an MR voxel, often a known, preferably linear, relationship between signal intensity change and concentration is used. However, in general this relation is non-linear for GBCAs and iron-oxides and is also dependent on the properties of the underlying tissue, magnetic field strength and temperature. In chapter 3, the image signal effects of a ionic iron compound $\left(\mathrm{FeCl}_{3}\right)$ are shown for a series of concentrations using $T_{1}$ and $T_{2}$ weighted gradient echo pulse sequences. Depending on the type of pulse sequence, for instance $T_{1}$ or $T_{2}$ weighted, the concentration of contrast medium can be visualized either as hypo- or hyperintense. For very low concentrations of iron particles there is an approximately linear signal elevating effect on the MR signal of $T_{1}$-weighted pulse sequences, while for $T_{2}$-weighted pulse sequences this effect strongly decreases the MRI signal in a non-linear way. This $T_{2}$-weighted effect caused by spin-spin dephasing shall also dominate the MR signal for $T_{1}$-weighted sequences at higher particle concentrations. Signal decreases due to the contrast medium do not only manifest at the precise location of the contrast particles but may also arise in their vicinity (i.e. blooming) due to the accompanying (local and remote) field inhomogeneities that lead to $T_{2}^{*}$ susceptibility effects. These non-linear contrast effects can make it very difficult to accurately quantify iron-oxides contrast medium concentrations, particularly when they are high. In chapter 2, a quantification method for the image effects of iron-oxides contrast media was therefore based on the area of enhanced voxels (EVs), largely ignoring the numerical changes in intensity. A low concentration of contrast media strongly reduces the risk of image artifacts, but decreases its detectability.

For fluorine, the relation between the signal intensity and the contrast media concentration is linear, under the assumption that the static magnetic field is constant and spin-density weighted pulse sequences are used. The relaxation time of fluorine contrast media still has a dependency on the temperature and tissue oxygenation. With a calibration phantom, which takes in account these factors, it may be possible to determine the in vivo concentration based on the spin density effect. Despite that a physical quantification with MR is possible, no studies have been published which report an in vivo measured concentration of fluorine contrast media. Some molecular fluorine MRI studies show the feasibility of in vivo detection and in vitro quantification $[4,21]$. A logical next step would be in vivo fluorine quantification, which can lead to interesting (pre-)clinical applications, such as quantification of phagocytosis of fluorine loaded nanoparticles, that accumulate in inflamed tissue ${ }^{[21-24]}$. Determining the concentration of a contrast medium in an atherosclerotic plaque may enable the quantification of plaque inflammation, which can be used to monitor changes over time for the guidance of inflammatory therapy ${ }^{[25]}$.

Chapter 5 describes an approach to visualize thrombogenesis with fluorine loaded nanoparticles as a direct contrast medium. The signal enhancement by the contrast medium indicates not only the presence of a fresh thrombus, but also its size due to the specific binding of the contrast medium to the rim of the thrombus. This contrast 
medium can lead to a specific quantification of the thrombus age and size, and could possibly lead to a patient specific treatment of the thrombus, whereas in the current clinical practice a treatment decision is based on the time duration since the onset of clinical symptoms. 


\subsection{Multi-spectral}

Molecular imaging is usually based on the visualization of a single biomolecular target. However, pathophysiologic features can generally be complex and with a single target the molecular signature of a disease may not be easily defined. Addressing multiple biological targets with molecular imaging may assist in the diagnosis that is based on more complex pathophysiologic processes.

In clinical diagnostic imaging, for instance computed-tomography (CT), X-ray imaging, ultra-sound (US) and MRI the anatomy is depicted and a contrast agent is visualized as a hypo- or hyperintense effect relative to the anatomic structures. For nuclear imaging techniques, positron emission tomography (PET) and single-photon emission computed tomography (SPECT), the contrast agent (i.e. tracer) is depicted and, if available merged, with an anatomic (CT or MR) image ${ }^{[26]}$. In nuclear imaging only SPECT allows the use of multi-spectral (gamma photons with various energies) tracers ${ }^{[27]}$.

With GBCAs or iron oxides, multi-spectral imaging is difficult, if not impossible. Different forms of GBCAs or iron oxides cannot be uniquely identified because they are indirect contrast agents, which interact with the surrounding (water or lipid) protons. The opportunity to use multi-spectral or hybrid ${ }^{1} \mathrm{H} /{ }^{19} \mathrm{~F}$ MR imaging is discussed in chapter 4 . In this chapter, a few examples are presented where both the anatomy and fluorine contrast medium is imaged in one MRI session. Fluorine has the advantage of a wide chemical shift, which makes it possible to identify different fluorine nuclei at different chemical positions by their shifted resonance frequencies. In an in vitro MRI study by Caruthers et al, it was shown that multiple fluorine contrast media could be visualized and targeted to multiple biological targets ${ }^{[28]}$. They used fluorine contrast media containing fluorine molecules with a small difference in resonance frequency.

Thus far, only a few pre-clinical studies have demonstrated the possibilities of multispectral imaging ${ }^{[28,29]}$. For fluorine based MRI high concentrations of contrast media are needed. Therefore, also a high concentration of ligand (peptide or antibody) has to realized. These high concentrations make the costs higher and increase the risk of side effects. Despite these remaining challenges, multi-spectral MR has great opportunities in medical imaging to identify various biological targets and their exact location in vivo with a minimal invasive procedure. 


\subsection{Future perspectives}

This thesis showed how novel contrast media can be applied in pre-clinical MRI studies on vascular pathology. Depending on the research question and application, imaging methods need to be tailored. Above all, a contrast medium must be measurable, which is a combination of sensitivity or detectability relative to the background signal or noise. For quantification purposes, linearity is beneficial, while multi-spectral acquisition opens opportunities for multi-biomarker imaging.

For iron-oxides and fluorine contrast media, improvements on the detectability can be made regarding the sensitivity of the MR acquisition, e.g. higher field strength or SNR improvement with dedicated multi-element coils. Also on the image reconstruction side better detectability can be achieved with dedicated image post-processing, e.g. SRR and SGM. For iron-oxides, the detectability is much better than for fluorine or GBCAs. Therefore, most studies using fluorine contrast media have to deal with the maximum amount of contrast media that can be administered and the maximum acquisition time that can realistically be achieved in a clinical setting. To improve or enable new pre-clinical ${ }^{19} \mathrm{~F} \mathrm{MRI}$ applications, the efficiency of the fluorine contrast media should be further improved. To increase the efficiency and to enable faster acquisition protocols for fluorine imaging, the longitudinal relaxation time can be lowered by using lanthanide(III) complexes ${ }^{[30,31]}$. Shortening of the longitudinal relaxation time can be achieved when a lanthanide(III) atom is incorporated in the fluorine particle or molecule. De Luca et al. showed in a pre-clinical study the in vivo use of Holmium-CF3 and Dyspro־sium-CF3 complexes with longitudinal relaxation times of 5-10 ms (at 9.4T) ${ }^{[31]}$. These developments are promising for future clinical applications, however other tradeoffs have to be taken into account, e.g. the inherently low fluorine density and the $R_{1} / R_{2}\left(=T_{2} / T_{1}\right)$ ratio. By decreasing the $T_{1}$, the $T_{2}$ will also decrease (without obvious changes in $R_{1} / R_{2}$ ) which will reduce the detectability. Fortunately, De Luca et al. mentioned a 10 - to 25 -fold increase in sensitivity with lanthanide-fluorine complexes compared to conventional fluorine imaging ${ }^{[31]}$. 


\subsection{Clinical perspectives}

Although the research in this thesis was of pre-clinical nature, the ultimate goal is clinical translation. In chapter 2, we showed that with labeled iron oxides particles and positive contrast techniques, the expression of molecular biomarkers of angiogenesis (CD13) could be visualized. Tumor angiogenesis is a sign of tumor malignancy and molecular MRI targeted to angiogenesis may provide a diagnosis with minimal invasive techniques. Furthermore, it may be used as a monitoring method during tumor therapy with angiogenesis inhibitors or cytostatic drugs ${ }^{[32,33]}$.

With the review in chapter 4 on ${ }^{19} \mathrm{~F} /{ }^{1} \mathrm{H}$ hybride imaging and the use of fluorine contrast media in chapters 5 and 6 the applicability of vascular (molecular) fluorine contrast media was presented. These studies show the strengths and weaknesses of fluorine imaging. Steps need to be taken to make fluorine imaging a mature imaging technique by further improving MR acquisition techniques and contrast media. The availability of fluorine contrast media for intravenous use is limited. No FDA approved fluorine contrast media exist yet, although in clinical trials fluorine is used for human cell tracking studies ${ }^{[34,35]}$. This thesis showed the diversity of fluorine contrast media and its potential for clinical applications, although numerous obstacles still need to be overcome.

This thesis focuses on imaging innovations with iron-oxides and fluorine based contrast media for vascular MRI. Human biosafety is the most critical issue for clinical translation of these contrast media. Both iron oxides and fluorine have already been applied successfully and safely in humans in the setting of clinical trials, see Table 7.2. Widespread use requires approval of regulatory bodies such as the FDA and EMA. Commercial development of these contrast agents depends on successful market introduction and market persistence, which depend on demand and (high) production costs, patents and long term safety ${ }^{[36,37]}$.

Table 7.2 - Human applicability of gadolinium, iron-oxides and fluorine

\begin{tabular}{|c|c|c|c|}
\hline Contrast medium & Administration & Clinical approved & Clinical usage \\
\hline Gadolinium & Intravenously & $\begin{array}{l}\text { Among others, } \\
\text { Magnevist, } \\
\text { Omniscan, and } \\
\text { Optimark [38] }\end{array}$ & In clinical use \\
\hline Iron-oxide & $\begin{array}{l}\text { Intravenously, Ex } \\
\text { vivo labeled cells }\end{array}$ & $\begin{array}{l}\text { Feridex, Resovist, } \\
\text { Ferumoxides }[39,40]\end{array}$ & $\begin{array}{l}\text { Used in clinical } \\
\text { trials }\end{array}$ \\
\hline${ }^{19}$ Fluorine & $\begin{array}{l}\text { Intravenously, Ex } \\
\text { vivo labeled cells, } \\
\text { Ventilation gas }\end{array}$ & $\begin{array}{l}\text { SonoVue (US } \\
\text { contrast medium) } \\
{[41] \text {, Fluosol*, }} \\
\text { Oxygent*, } \\
\text { Perftoran }{ }^{*}[42]\end{array}$ & Experimental \\
\hline
\end{tabular}

*Only used in clinical trails 


\subsection{Conclusion}

In this thesis iron-oxides and fluorine contrast media were demonstrated as novel contrast agents for MR angiography and molecular MR imaging of angiogenesis and thrombus expression. In preclinical tumor models, molecular imaging of angiogenesis was demonstrated using a combination of iron oxides nanoparticles and positive contrast post-processing techniques. In a preclinical thrombus model, contrast enhancement after injection of fluorine loaded nanoparticles was shown. In this thrombus model, a novel method was used to induce thrombi which reduces the risk of susceptibility artifacts in MRI. For fluorine imaging, imaging reconstruction techniques were compared to shorten the acquisition time.

The results of the studies show how iron-oxides and fluorine contrast media can extend on the possibilities of GBCAs regarding background suppression, detectability, linearity and multi-spectral potential. Although some of these contrast media are on the verge to enter clinical applications, further developments are still necessary. For iron oxides the focus should be on optimizing positive contrast techniques and relevant clinical molecular imaging applications. For fluorine contrast media, increasing the detectability is paramount to successfully realize clinical vascular MRI applications. 


\section{References}

1. Hanaoka, K., et al., The detection limit of a Gd3+-based $T$, agent is substantially reduced when targeted to a protein microdomain. Magn Reson Imaging, 2008. 26(5): p. 608-17.

2. Morawski, A.M., et al., Targeted nanoparticles for quantitative imaging of sparse molecular epitopes with MRI. Magn Reson Med, 2004. 51(3): p. 480-6.

3. Heyn, C., et al., Detection threshold of single SPIO-labeled cells with FIESTA. Magn Reson Med, 2005. 53(2): p. 312-20.

4. van Heeswijk, R.B., et al., Fluorine-19 magnetic resonance angiography of the mouse. PLoS One, 2012. 7(7): p. e42236.

5. Shapiro, E.M., et al., MRI detection of single particles for cellular imaging. Proc Natl Acad Sci U S A, 2004. 101(30): p. 10901-6.

6. Gore, J.C., et al., Molecular imaging without radiopharmaceuticals? J Nucl Med, 2009. 50(6): p. 999-1007.

7. Seppenwoolde, J.H., M.A. Viergever, and C.J. Bakker, Passive tracking exploiting local signal conservation: the white marker phenomenon. Magn Reson Med, 2003. 50(4): p. 784-90.

8. Dahnke, H., et al., Susceptibility gradient mapping (SGM): a new postprocessing method for positive contrast generation applied to superparamagnetic iron oxide particle (SPIO)labeled cells. Magn Reson Med, 2008. 60(3): p. 595-603.

9. Stuber, M., et al., Positive contrast visualization of iron oxide-labeled stem cells using inversion-recovery with ON-resonant water suppression (IRON). Magn Reson Med, 2007. 58(5): p. 1072-7.

10. Mani, V., et al., Gradient echo acquisition for superparamagnetic particles with positive contrast (GRASP): sequence characterization in membrane and glass superparamagnetic iron oxide phantoms at 1.5T and 3T. Magn Reson Med, 2006. 55(1): p. 126-35.

11. Dharmakumar, R., I. Koktzoglou, and D. Li, Generating positive contrast from off-resonant spins with steady-state free precession magnetic resonance imaging: theory and proof-ofprinciple experiments. Phys Med Biol, 2006. 51(17): p. 4201-15.

12. Cukur, T., et al., Positive contrast with alternating repetition time SSFP (PARTS): a fast imaging technique for SPIO-labeled cells. Magn Reson Med, 2010. 63(2): p. 427-37.

13. Faber, C., et al., Sensitivity to local dipole fields in the CRAZED experiment: an approach to bright spot MRI. J Magn Reson, 2006. 182(2): p. 315-24.

14. Ciciliano, J.C., et al., Resolving the multifaceted mechanisms of the ferric chloride thrombosis model using an interdisciplinary microfluidic approach. Blood, 2015. 126(6): p. 817-24.

15. Starmans, L.W., et al., Evaluation of iron oxide nanoparticle micelles for magnetic particle imaging (MPI) of thrombosis. PLoS One, 2015. 10(3): p. e0119257.

16. de Vries, A., et al., Relaxometric studies of gadolinium-functionalized perfluorocarbon nanoparticles for MR imaging. Contrast Media Mol Imaging, 2014. 9(1): p. 83-91.

17. Greenspan, $\mathrm{H}_{\text {., et }}$ al., MRI inter-slice reconstruction using super-resolution. Magn Reson Imaging, 2002. 20(5): p. 437-46. 
18. Gholipour, A., J.A. Estroff, and S.K. Warfield, Robust super-resolution volume reconstruction from slice acquisitions: application to fetal brain MRI. IEEE Trans Med Imaging, 2010. 29(10): p. 1739-58.

19. Poot, D.H., et al., Super-resolution for multislice diffusion tensor imaging. Magn Reson Med, 2013. 69(1): p. 103-13.

20. Van Steenkiste, G., et al., Super-resolution $T_{1}$ estimation: Quantitative high resolution $T_{1}$ mapping from a set of low resolution $T_{1}$-weighted images with different slice orientations. Magn Reson Med, 2016.

21. Flogel, U., et al., In vivo monitoring of inflammation after cardiac and cerebral ischemia by fluorine magnetic resonance imaging. Circulation, 2008. 118(2): p. 140-8.

22. Bonner, F., et al., Multifunctional MR monitoring of the healing process after myocardial infarction. Basic Res Cardiol, 2014. 109(5): p. 430.

23. Temme, S., et al., ${ }^{19} \mathrm{~F}$ magnetic resonance imaging of endogenous macrophages in inflammation. Wiley Interdiscip Rev Nanomed Nanobiotechnol, 2012. 4(3): p. 329-43.

24. Ahrens, E.T. and J.W. Bulte, Tracking immune cells in vivo using magnetic resonance imaging. Nat Rev Immunol, 2013. 13(10): p. 755-63.

25. van Heeswijk, R.B., et al., Fluorine MR Imaging of Inflammation in Atherosclerotic Plaque in Vivo. Radiology, 2015. 275(2): p. 421-9.

26. Prior, J.O., H. Farhad, and O. Muller, Multimodality Imaging in Ischemic Cardiomyopathy. Curr Cardiovasc Imaging Rep, 2014. 7: p. 9285.

27. Guo, Z., et al., Simultaneous SPECT imaging of multi-targets to assist in identifying hepatic lesions. Sci Rep, 2016. 6: p. 28812.

28. Caruthers, S.D., et al., In vitro demonstration using ${ }^{19} \mathrm{~F}$ magnetic resonance to augment molecular imaging with paramagnetic perfluorocarbon nanoparticles at 1.5 Tesla. Invest Radiol, 2006. 41(3): p. 305-12.

29. Jacoby, C., et al., Probing different perfluorocarbons for in vivo inflammation imaging by ${ }^{19} \mathrm{~F}$ MRI: image reconstruction, biological half-lives and sensitivity. NMR Biomed, 2014. 27(3): p. 261-71.

30. Nakamura, T., et al., Activatable ${ }^{19} \mathrm{~F}$ MRI nanoparticle probes for the detection of reducing environments. Angew Chem Int Ed Engl, 2015. 54(3): p. 1007-10.

31. De Luca, E., et al., Characterisation and evaluation of paramagnetic fluorine labelled glycol chitosan conjugates for (19)F and (1) $\mathrm{H}$ magnetic resonance imaging. J Biol Inorg Chem, 2014. 19(2): p. 215-27.

32. Garcia-Figueiras, R., A.R. Padhani, and S. Baleato-Gonzalez, Therapy Monitoring with Functional and Molecular MR Imaging. Magn Reson Imaging Clin N Am, 2016. 24(1): p. 261-88.

33. Wick, W., et al., Current status and future directions of anti-angiogenic therapy for gliomas. Neuro Oncol, 2016. 18(3): p. 315-28.

34. Rose, L.C., et al., Fluorine-19 Labeling of Stromal Vascular Fraction Cells for Clinical Imaging Applications. Stem Cells Transl Med, 2015. 4(12): p. 1472-81.

35. Ahrens, E.T., et al., Clinical cell therapy imaging using a perfluorocarbon tracer and fluorine-19 MRI. Magn Reson Med, 2014. 72(6): p. 1696-701. 
36. Lexchin, J., How safe are new drugs? Market withdrawal of drugs approved in Canada between 1990 and 2009. Open Medicine: A Peer-reviewed, Independent, Open-access Journal, 2014. 8(1): p. e14-9.

37. Munos, B., 2015 New Drug Approvals Hit 66-Year High! Forbes, 2016.

38. Taupitz, M., et al., Gadolinium-containing magnetic resonance contrast media: investigation on the possible transchelation of $\mathrm{Gd}(3)(+)$ to the glycosaminoglycan heparin. Contrast Media Mol Imaging, 2013. 8(2): p. 108-16.

39. Korchinski, D.J., et al., Iron Oxide as an MRI Contrast Agent for Cell Tracking. Magn Reson Insights, 2015. 8(Suppl 1): p. 15-29.

40. Finn, J.P., et al., Cardiovascular MRI with ferumoxytol. Clin Radiol, 2016. 71(8): p. 796-806.

41. Amiri, H., et al., Cell tracking using (19)F magnetic resonance imaging: technical aspects and challenges towards clinical applications. Eur Radiol, 2015. 25(3): p. 726-35.

42. Castro, C.I. and J.C. Briceno, Perfluorocarbon-based oxygen carriers: review of products and trials. Artif Organs, 2010. 34(8): p. 622-34. 


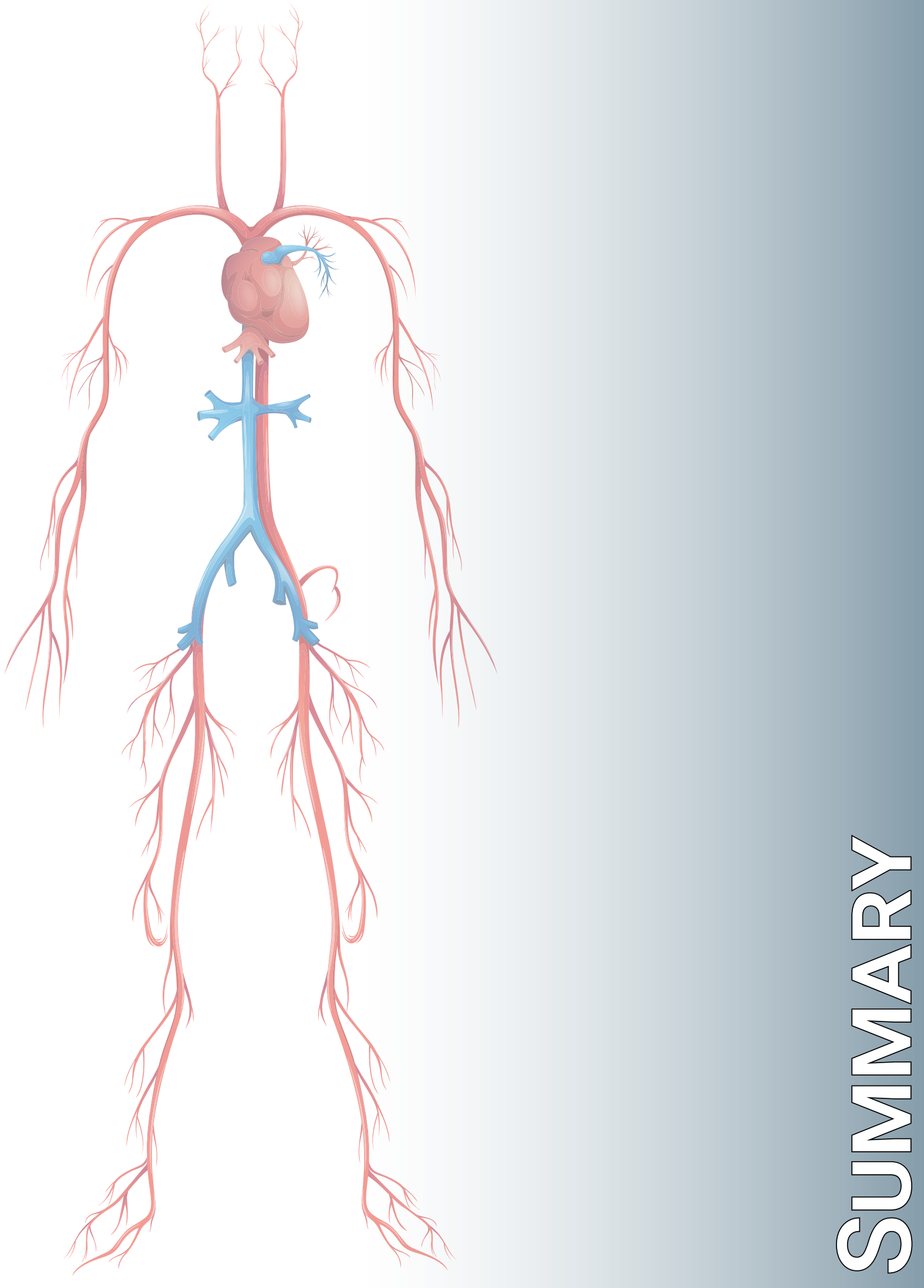




\section{Summary}

Magnetic Resonance Imaging (MRI) is currently one of the most versatile (noninvasive) medical imaging techniques in vascular diagnostics. MRI provides high spatial resolution and superior soft-tissue contrast. This allows visualization of small details and differentiation between different tissues types without the use of ionizing radiation. Additionally, it provides the ability to perform quantitative measurements, for example, to evaluate the effect of therapy. The versatility of MRI can be extended with the use of contrast agents. Contrast-enhanced MRI is beneficial for a wide range of clinical applications, including vascular MRI to enhance the contrast between the lumen and surrounding tissue. The majority of the contrast agents that are used for MRI are gadolinium-based contrast agents (GBCAs). In spite of the great advantages of GBCAs, there are some drawbacks. These drawbacks relate to 1) the strong background signal of the (native) tissue, 2) the poor detectability of the contrast agent at very low concentrations, 3 ) the non-linearity between the signal intensity and the concentration of the contrast agent, and 4) the impossibility to (spectrally) distinct multiple GBCAs which are chemically different. In this thesis, the applicability of alternative contrast agents, i.e. iron oxide and fluorine-based contrast agents, was investigated for vascular MRI. We explored whether the disadvantages of GBCAs can be overcome with these alternative contrast agents for vascular MRI.

Chapter 1 provides a general introduction on the MRI contrast mechanisms of GBCAs, iron-oxide contrast agents, and fluorine-based contrast agents.

In Chapter 2, we present a molecular imaging study using superparamagnetic iron oxide particles (SPIOs) and positive contrast techniques. Positive contrast techniques transform a conventional negative contrast (dark region in image) into a positive contrast (bright region in image). Two different positive contrast techniques, i. e. "White Marker" (WM) and "Susceptibility Gradient Mapping" (SGM), were evaluated for molecular MRI of tumor angiogenesis, i.e. the formation of new small blood vessels in a tumor. We compared WM and SGM with a conventional gradient echo (GE) imaging technique, which provides a negative contrast. In this study, tumor-bearing mice were used as a model for tumor angiogenesis. The mice were injected with either unlabeled SPIOs or SPIOs labeled with the cyclic NGR peptide, which specifically binds to angiogenic microvessels. After obtaining conventional and positive contrast images, the percentage of contrast-enhanced voxels in the tumor were determined as a (surrogate) measure of the amount of contrast agent (indicative for new small blood vessels). The image analysis showed a significantly higher amount of contrast agent in the tumor edge in comparison to the tumor core region. In the present experimental design, only SGM (and not WM) was able to convert the negative contrast of the SPIO contrast agent into a detectable positive contrast signal.

In preclinical thrombus formation studies, a thrombus in an animal model is often induced by the use of iron chloride $\left(\mathrm{FeCl}_{3}\right)$ solutions. Chapter 3 shows that remnants (residuals) of $\mathrm{FeCl}_{3}$ used in the animal model give rise to artifacts in the MR image, which is shown as a negative contrast. In the vicinity of a thrombus, the presence of $\mathrm{FeCl}_{3}$ can cause hypo- or hyperintensity artifacts in the MR image. These artifacts 
complicate the interpretation of contrast effects in molecular MRI studies expected by a thrombus-bound contrast agent. The use of (non-ferromagnetic) aluminum chloride $\left(\mathrm{AlCl}_{3}\right)$ can serve as an alternative because it gives no such image artifacts. We demonstrated that the effect of $\mathrm{FeCl}_{3}$ and $\mathrm{AlCl}_{3}$ is comparable in terms of thrombus formation. Low concentrations of $\mathrm{AlCl}_{3}$ do not cause image artifacts for MRI.

The number of applications of fluorine $\left({ }^{19} \mathrm{~F}\right) \mathrm{MR}$ imaging and spectroscopy in biomedical and clinical research is growing steadily. Fluorine-based contrast agents have specific advantages over conventional contrast agents such as gadolinium or iron oxide based contrast agents. The low and negligibly low concentration of fluorine in the human body, facilitates MR detection and reliable quantification of fluorinebased contrast agents or fluorine-containing drugs. At the same time, the anatomy can be visualized using proton MRI by the application of hybrid ${ }^{1} \mathrm{H} /{ }^{19} \mathrm{~F}$ imaging. Preclinical studies showed new developments in the field of fluorine contrast agents and imaging techniques. These techniques are promising for future clinical applications. Chapter 4 provides a literature review of these new developments. This chapter discusses the opportunities and future prospects of translating pre-clinical MR imaging and spectroscopy hybrid ${ }^{1} \mathrm{H} /{ }^{19} \mathrm{~F}$ MRI applications to the clinic.

Fluorine-based molecular MRI may improve the diagnosis of acute blood clots by making use of fibrin-specific contrast media. The MR signal from the fluorine contrast medium is detected directly, and not hampered by background signal from surrounding tissue or blood (as with proton MRI). The feasibility of MRI of thrombi with a fluorine-loaded nanoparticle labeled with the fibrin-specific peptide A14 is described in Chapter 5. This new fibrin specific fluorine nanoparticle shows specific affinity for fresh thrombi, as demonstrated with ex vivo and in vitro fluorine MRI measurements. However, further development is required for in vivo imaging with this nanoparticle, focusing on a higher sensitivity of this fluorine MRI application.

The absence of tissue background signal for fluorine-based contrast media is very useful in MR angiography. However, a fluorine MRI acquisition often requires a lot of time due to the relatively long $T$, relaxation time of fluorine and the intrinsically low signal-to-noise ratio. In Chapter 6, we examined the use of fast multi-slice image acquisitions in combination with super-resolution reconstruction (SRR) techniques in order to shorten the acquisition time for fluorine MRI, while maintaining high spatial resolution. The study focused on three SRR techniques, namely "Iterative Back Projection" (IBP), "Robust Iterative Back Projection" (RIBP), and linear interpolation (INT). These techniques were compared with conventional 3D acquisitions, under conditions with a low signal-to-noise ratio, which is characteristic for fluorine MR angiography. The study evaluates the performance of the three SRR techniques in terms of signal-to-noise ratio and spatial resolution. When acquisition time is no issue, a 3D MRI shows most details in fluorine MR angiography. At shorter acquisition times however, RIBP showed good performance in terms of spatial resolution when sufficient input SNR is provided. INT provides overall the highest gain in SNR, and thus contrast resolution, which is advantageous over native 3D MRI acquisitions by reduction of acquisition time.Depending on the specific image (quality) requirements, 
one of these SRR techniques can be chosen for either an optimal signal-to-noise ratio (INT), spatial resolution (IBP), or a compromise of these two image properties (RIBP).

The findings of the previous chapters are discussed in a broader perspective in Chapter 7. This final chapter describes which obstacles need to be overcome for the translation of these new acquisition techniques and contrast agents for future clinical applications. 


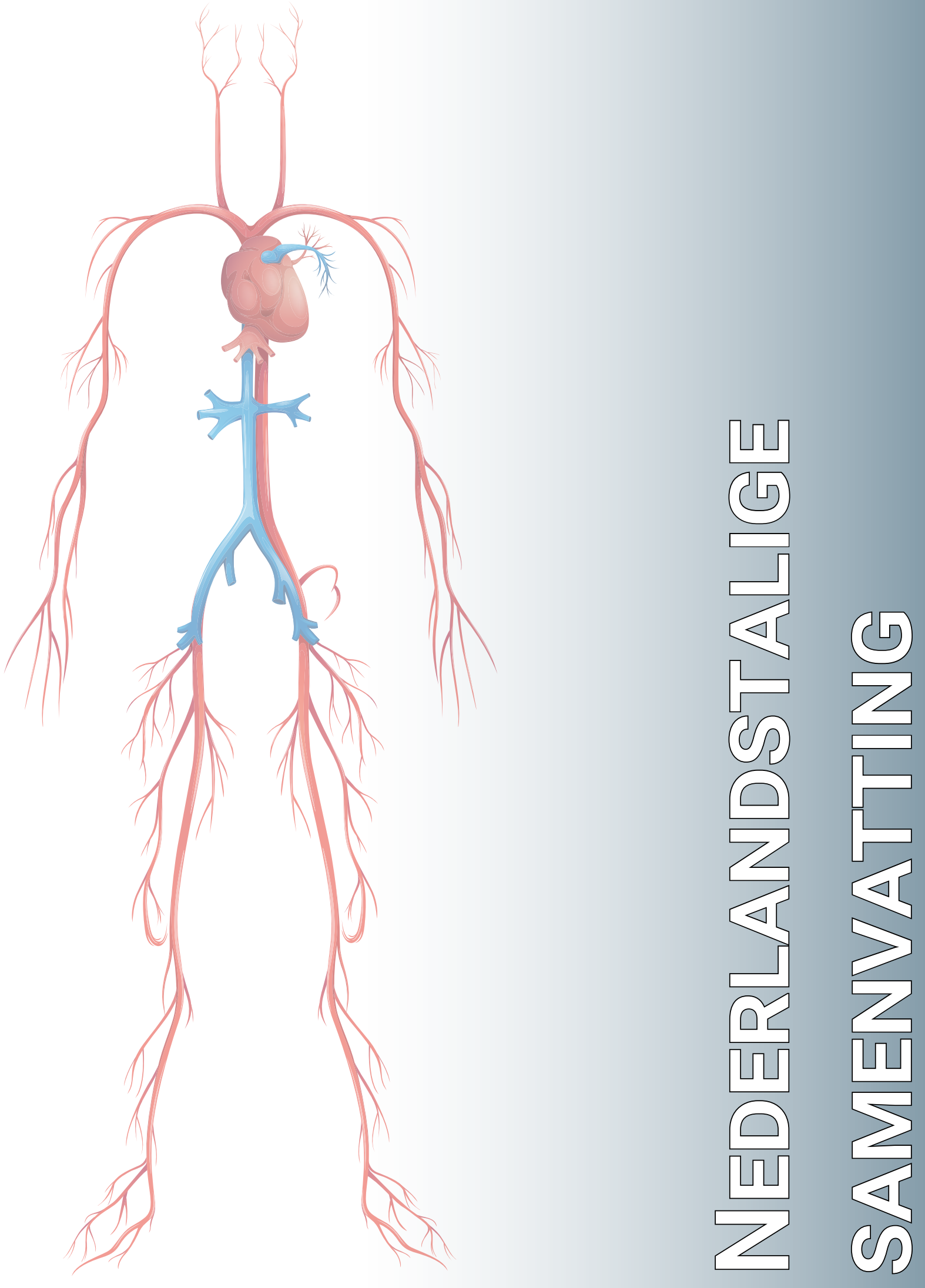




\section{Samenvatting}

Magnetische Resonantie Imaging (MRI) is momenteel één van de meest veelzijdige (non-invasieve) medische beeldvormende technieken voor vasculaire diagnostiek. MRI voorziet in een hoge spatiële resolutie, waardoor kleine details in beeld gebracht kunnen worden, een superieur zacht-weefsel contrast, zodat het mogelijk is om verschillende weefsels te onderscheiden en maakt geen gebruik van ioniserende straling. Daarnaast biedt het de mogelijkheid om kwantitatieve metingen uit te voeren, bijvoorbeeld om het effect van therapie te evalueren op basis van beschrijvende getallen. De veelzijdigheid van MRI kan verder worden uitgebreid met het gebruik van contrastmiddelen om het contrast te versterken tussen het lumen, het inwendige van het bloedvat waar het bloed doorheen stroomt, en het omringende normale of aangedane weefsel. Voor contrastversterking ten behoeve van een breed scala aan klinische toepassingen, waaronder vasculaire MRI, wordt vrijwel altijd een op gadolinium gebaseerd contrastmiddel (GBCM) gebruikt. Ondanks de grote voordelen van een GBCM, zijn er nadelen aan dit contrastmiddel. Deze nadelen hebben betrekking op 1) het sterke achtergrondsignaal van het weefsel, 2) de moeizame detecteerbaarheid van het contrastmiddel bij zeer lage concentraties, 3) de nonlineaire relatie tussen de signaalintensiteit en de concentratie van het contrastmiddel en 4) het niet kunnen onderscheiden van contrastmiddelen die chemisch verschillend zijn. In dit proefschrift is de toepasbaarheid van alternatieve nieuwe contrastmiddelen, te weten ijzeroxide en fluor-gebaseerde contrastmiddelen, voor vasculaire MRI onderzocht. Er is onderzocht of met deze contrastmiddelen specifieke nadelen van een GBCM, die zich manifesteren tijdens MRI diagnostiek van vasculaire pathologie, kunnen worden vermeden.

In Hoofdstuk 1 wordt een algemene introductie gegeven over het contrastmechanisme van gadolinium-, ijzeroxide- en fluor-gebaseerde contrastmiddelen.

In Hoofdstuk 2 presenteren we moleculaire beeldvorming met behulp van superparamagnetische ijzeroxide deeltjes (SPIOs) en positieve contrast technieken. Positieve contrast technieken transformeren een conventioneel negatief contrast (donkere regio in het beeld) in een positief contrast (lichtere regio in het beeld). Twee verschillende positieve contrasttechnieken, "White Marker" (WM) en "Susceptibility Gradient Mapping" (SGM), werden geëvalueerd voor moleculaire MRI van tumor angiogenese, dat wil zeggen de nieuwvorming van kleine bloedvaatjes in een kankergezwel. We vergeleken deze positieve contrast technieken met conventionele gradiënt echo (GE) beeldvorming, dat een negatief contrast geeft.

In deze studie zijn tumor-dragende muizen gebruikt als model voor angiogenese. De muizen werden geïnjecteerd met ongelabelde SPIOs of SPIOs gelabeld met het cyclische NGR peptide, dat specifiek bindt aan de angiogene microvaten. Na het verkrijgen van conventionele en positieve contrast beelden werd het percentage contrast-versterkte voxels in de tumor bepaald als (surrogaat) maat voor de hoeveelheid aan contrastmiddel (en dus nieuwe kleine bloedvaten). De beeldanalyse toonde een significant hoger contrast in de tumorrand, waar zich de meeste angiogene microvaatjes bevinden, ten opzichte van de kern. Van de positieve contrast technieken 
bleek, in deze proefopzet, alleen SGM (en niet WM) in staat om het negatieve contrast van het SPIO contrastmiddel om te zetten naar een detecteerbaar positief contrast.

In preklinische studies naar trombusvorming wordt vaak een diermodel toegepast waarin een trombus door middel van een ijzerchloride $\left(\mathrm{FeCl}_{3}\right)$ oplossing wordt geïnduceerd. Hoofdstuk 3 laat zien dat restanten van het gebruikte $\mathrm{FeCl}_{3}$ in het diermodel artefacten (met een negatief contrast) kunnen veroorzaken in het MRI beeld: $\mathrm{FeCl}_{3}$ kan hypo- of hyper-intensiteiten veroorzaken in de nabijheid van een trombus. Dit betekent dat de artefacten soms leiden tot te veel en soms tot te weinig signaal. Dit compliceert bijvoorbeeld de interpretatie van het contrast effect van een aan trombus gebonden contrastmiddel in moleculaire MRI studies. Het gebruik van (non-ferromagnetisch) aluminium-chloride $\left(\mathrm{AlCl}_{3}\right)$ kan dienen als een alternatief, omdat het geen MRI beeldartefacten geeft. We laten zien dat het effect van $\mathrm{FeCl}_{3}$ en $\mathrm{AlCl}_{3}$ qua trombusvorming vergelijkbaar is en er geen MRI artefacten optreden bij lage concentraties aan $\mathrm{AlCl}_{3}$.

Het aantal toepassingen van fluor $\left({ }^{19} \mathrm{~F}\right) \mathrm{MR}$-beeldvorming en spectroscopie groeit gestaag in biomedisch en klinisch onderzoek. Fluor-gebaseerde contrastmiddelen hebben specifieke voordelen ten opzichte van conventionele contrastmiddelen die op gadolinium of ijzeroxide gebaseerd zijn. De geringe en nagenoeg met MRI onzichtbare concentratie van fluor in het menselijk lichaam vergemakkelijkt beeldvorming en betrouwbare kwantificatie van fluor-gebaseerde contrastmiddelen of fluorhoudende geneesmiddelen. Daarnaast kan met proton MRI tegelijkertijd de anatomie worden gevisualiseerd door toepassing van hybride ${ }^{1} \mathrm{H} /{ }^{19} \mathrm{~F}$ beeldvorming. In preklinische studies hebben nieuwe ontwikkelingen plaatsgevonden op het gebied van fluorhoudende contrastmiddelen en beeldvormingstechnieken. Deze technieken lijken veelbelovend voor toekomstige klinische toepassingen. Hoofdstuk 4 geeft een literatuuroverzicht van deze nieuwe ontwikkelingen. Dit hoofdstuk bespreekt de perspectieven voor translatie van preklinische hybride ${ }^{1} \mathrm{H} /{ }^{19} \mathrm{~F}$ MR beeldvorming en spectroscopie naar toekomstige toepassingen in de kliniek.

Fluor-gebaseerde moleculaire MRI kan mogelijk de diagnose van acute bloedstolsels verbeteren door gebruik te maken van fibrine specifieke contrastmiddelen. Het MRI signaal van het fluorhoudende contrastmiddel wordt direct gedetecteerd, en wordt niet gehinderd door achtergrondsignaal van ander omringend weefsel (of bloed). In Hoofdstuk 5 wordt de haalbaarheid van moleculaire MRI beschreven van trombi met een fluorbevattend nanodeeltje gelabeld met het fibrine-specifieke A14 peptide. Dit nieuwe fibrine-specifieke fluorhoudend nanodeeltje toont specifieke affiniteit voor verse trombi, zoals aangetoond met ex vivo en in vitro fluor MRI metingen. Echter, voor in vivo beeldvorming met dit nanodeeltje zijn verdere ontwikkelingen vereist, toegespitst op een hogere gevoeligheid van deze specifieke fluor MRI toepassing.

De afwezigheid van weefsel achtergrond signaal maakt dat fluor-gebaseerde contrastmiddelen ook voor MR angiografie nuttig kunnen zijn. Echter, een fluor MRI 
acquisitie vergt vaak veel tijd door de relatief lange $T_{1}$ relaxatietijd van fluor en het (intrinsiek) lage signaal-ruis niveau.

In Hoofdstuk 6 onderzochten we snelle multi-slice acquisitie technieken in combinatie met super resolutie reconstructie (SRR) technieken om de acquisitietijd voor fluor MRI te verkorten met behoud van hoge spatiële resolutie om voldoende details te kunnen zien. Het onderzoek richtte zich op drie SRR technieken, namelijk "Iterative Back Projection" (IBP), "Robust Iteratief Back Projection" (RIBP) en lineaire interpolatie (INT). Deze technieken werden vergeleken met conventionele 3D acquisities, onder condities met een lage signaal-ruis verhouding, zoals kenmerkend is voor fluor MR angiografie.

De studie laat de prestaties van de drie SRR technieken zien voor verschillende signaalruis verhoudingen en spatiële resoluties. Wanneer de acquisitietijd een ondergeschikte rol speelt zal een 3D fluor MR angiografie de meeste details kunnen geven. Voor relatief korte acquisitietijden zal RIBP goede een spatiele resolutie leveren mits de SNR van de multi-slice acquisitie voldoende is. De hoogste SNR opbrengst en contrast resolutie wordt bereikt met INT, dit is voornamelijk voordeling om de acquisitietijd te reduceren in vergelijking tot conventionele 3D MRI acquisities. Op basis van specifieke afbeeldingswensen kan worden gekozen voor een optimale signaal-ruis verhouding (INT), spatiele resolutie (IBP) of een compromis van beide beeldeigenschappen (RIBP).

De bevindingen van de voorgaande hoofdstukken worden in Hoofdstuk 7 in een breder perspectief geplaatst. In dit laatste hoofdstuk wordt beschreven welke obstakels nog moeten worden overwonnen voor de translatie van deze nieuwe contrastmiddelen en acquisitietechnieken naar toekomstige klinische toepassingen. 


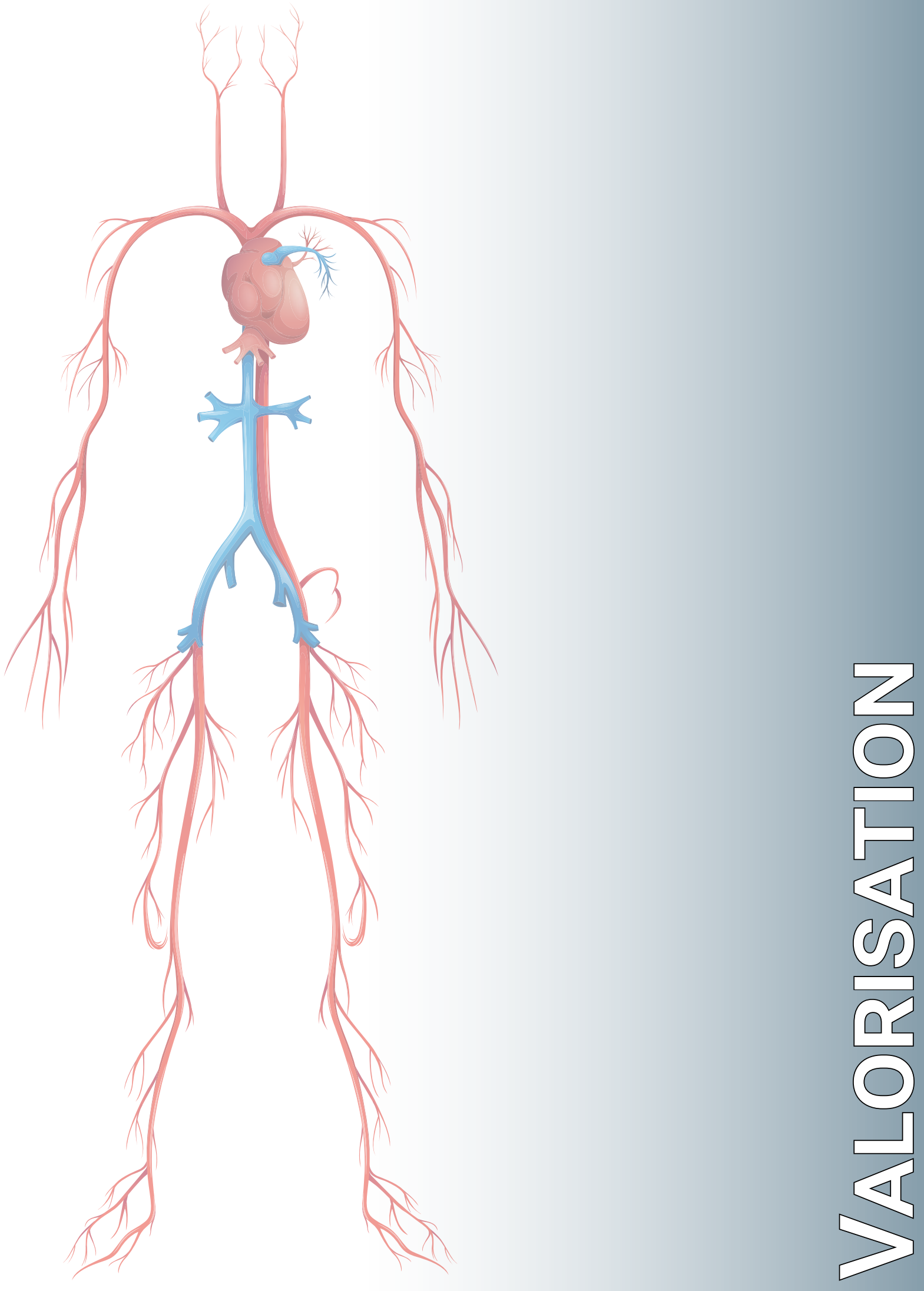




\section{Relevance of the study \\ Clinical healthcare problem}

Diseases of the heart and blood vessels, so-called cardiovascular diseases, are the number one cause of death worldwide. The World Health Organization estimated that 17.5 million people died from cardiovascular diseases in 2012. In the Netherlands, 8.3 billion euro was spent on the treatment of cardiovascular diseases in 2011, which was $9.2 \%$ of the total health care costs. By minimizing behavioral risk factors such as alcohol and tobacco use, obesity, unhealthy diet and physical inactivity, the risk for cardiovascular diseases can be reduced, but not eliminated. Patients who already developed clinical symptoms or have more than one cardiovascular risk factor may benefit from cardiovascular imaging by early detection of cardiovascular disease and identification of the disease stage, followed by appropriate treatment.

Diagnostic imaging of cardiovascular disease should, like every diagnostic test, have a high sensitivity and specificity. Ideally, the method is fast and should be able to identify early signs of cardiovascular disease, in a stage where the disease process may still be reversible. Examples of abnormal pathophysiology of the vascular system that can be imaged include atherosclerosis, which is a main underlying cause of myocardial infarction, cerebrovascular stroke and abnormal vessel development, and angiogenesis, which is an indication of disease progression in malignant tumors.

The current clinical angiographic techniques are able to visualize the large blood vessels very well. With or without contrast enhancement these techniques depict the lumen of the blood vessels (luminography). However, diagnosing the pathophysiology of the vascular disease is limited, as for instance the age of the thrombus that caused a cerebrovascular stroke or the degree of tumor angiogenesis, cannot be determined. To enable a next step in cardiovascular diagnosis, and to facilitate personalized treatment and monitoring of therapeutic responses, molecular markers may play a big role by specifically concentrating of contrast media to the pathophysiologic site. The same technology might be used to direct therapeutic agents, ideally combined with a diagnostic agent, an approach commonly referred to as theragnostics. The current clinically available nuclear imaging techniques, which are based on targeting of radioactively labeled molecular markers, are already highly suitable to visualize alterations in receptor expression, but do not provide a detailed anatomical (soft-tissue) image. To address this problem, clinical hybrid PET/CT and PET/MRI systems have been introduced. Such hybrid imaging modalities enable a single-stop-exam for the patient. However, a disadvantage of these nuclear imaging modalities is the need for radioactive pharmaceuticals, which makes it less suitable for screening and repeated follow-up studies. Therefore, there is a need for other approaches in diagnostic vascular imaging, for which MRI seems an ideal candidate. MRI does not use ionizing radiation, provides excellent soft tissue contrast and, in theory, is capable of using different types of contrast media to localize different molecular markers. Gadolinium-based contrast agents (GBCAs) are commonly used due to their good contrast enhancement and the relative low risks of adverse side effects. However, GBCAs cannot be used in patients with severely impaired kidney function because of the risk of nephrogenic systemic fibrosis (NSF). In addition, depositions of GBCAs in the brain and bones of patients, 
even those with normal renal function, were documented, although no short or longterm adverse effects of the low concentration of the deposition have been observed yet ${ }^{[1-3]}$. Furthermore, contrast enhancement properties of GBCAs are not optimal for molecular MRI. Novel concepts of contrast media and associated advanced MR imaging techniques are needed for molecular MRI.

\section{Approach}

For MR angiography, different methods are available to increase the contrast between the vascular lumen and surrounding tissue. In addition, molecular MRI can highlight disease-specific molecular targets of the vascular wall. Despite the wide clinical use of GBCAs, other MR contrast media could have specific advantages for vascular MRI. In certain applications such as imaging small objects like small blood vessels, or when the target tissue region is very small and hypodense as in some molecular imaging applications, the signal to background ratio with GBCAs can become critically low.

Fluorine and iron-oxide contrast media are alternatives for vascular MRI in molecular imaging and angiographic applications. Fluorine contrast media can be visualized without generating any signal from the background tissue. Iron-oxides are known for the strong contrast effect, which is typically much higher than for GBCAs.

Although fluorine contrast media require no background suppression due to the absence of background signal from tissue, signal levels are relatively low with respect to proton MR, which requires longer acquisition times to generate sufficient signal. Iron-oxide based contrast agents on the other hand, generate a strong image contrast, which however is usually a negative (hypointense) contrast effect. This thesis studies the feasibility of fluorine and iron-oxide, as alternative vascular MRI contrast media, and complementary MR imaging techniques.

\section{Main findings}

A fluorine contrast medium was used for MR angiography to benefit from the absence of tissue background signal. Due to the intrinsically low signal levels of fluorine $\mathrm{MR}$, the acquisition time is usually long, presenting practical problems with long stay of patients in the MRI scanner. In this thesis, a conventional MR sequence for fluorine MR was used, followed by super-resolution reconstruction post-processing techniques. These post-processing techniques enable to reduce the acquisition time, while maintaining at least the same spatial resolution and signal-to-noise ratio with respect to a conventional 3D fluorine MR acquisition. The reduction of scan time is an important factor to facilitate clinical translation.

The use of iron-oxides was applied to visualize the growth of novel blood vessels, i.e. angiogenesis, in fast growing tumors. Iron-oxides usually demonstrate a hypointense contrast (i.e. signal loss) on regular MR images. We showed that a positive contrast technique can be used to depict iron-oxide contrast hyperintensities and to highlight regions of angiogenesis. Positive contrast enhancement methods can be applied as image post-processing techniques on conventional diagnostic images that depict the 
anatomy, which means that the application does not increase the examination time. This is a strong advantage and an important factor for clinical translation.

In this thesis targeted fluorine nanoparticles were applied as a contrast medium to visualize ex vivo thrombi. The molecular target, a-2-antiplasmin, is only active when a thrombus is probably still susceptible to thrombolysis, and is therefore expected to be a good indicator for the decision to treat a blood clot or not. In ex vivo MRI measurements the fluorine a-2-antiplasmin targeted contrast medium could differentiate specifically targeted and control targeted uptake. The in vivo detection of the targeted and control targeted contrast media remains challenging and requires further improvements before it can used.

\section{Target population}

The future target population of the current research work are patients that suffer from cardiovascular diseases. In this thesis, it was shown that advanced contrast agents can be used to study the normal and diseased vasculature. The advantage of fluorine contrast media to depict the vasculature, separately from the organs and other structures, is that it enables a clear delineation of the vessel lumen (where contrast resides) from the background signal. Also the applicability of molecular targeted contrast media, for detection of blood clots or angiogenesis, is potentially beneficial for future patients. Eventually, both fluorine and iron-oxide contrast media may contribute to more sensitive and more specific detection and visualization of pathological processes, even before symptoms or structural abnormalities can be noticed. Furthermore, the possibility to visualize the anatomy and molecular targeted contrast media in a "one-stop shop" examination and the absence of ionizing radiation makes MR imaging attractive for screening and follow-up procedures.

The studies of this thesis and its results are also relevant for the healthcare industry. On the one side for the hospitals in terms of cost reduction (reduced acquisition time) and on the other side for the pharmaceutical industry regarding development of novel contrast media and also vendors of MRI systems and image processing software. Hospitals could benefit from the use of iron-oxide contrast media for clinical molecular MR imaging, because of its high sensitivity. This diagnostic exam can result in additional value in the same MR session and therefore save on equipment and/or time on a multi-modality diagnostic imaging with PET or SPECT. Naturally, this has to be weighed against the additional costs of the contrast media and this is where the development of contrast media suitable for molecular MR imaging becomes interesting for the healthcare industry. MR imaging is suitable for screening and follow-up studies, due to the lack of ionizing radiation, but well-developed clinical applications for molecular imaging are still lacking.

In clinical research there is a shift towards use of (ultra-)high-field MR systems, which may provide new applications for advanced contrast media and diagnostic imaging. However, the current generation GBCAs have a low efficiency at high-field. The novel 
contrast media used in this thesis will benefit from a stronger magnetic field and will therefore be even more valuable in (ultra-)high-field MRI applications.

\section{Innovation and future}

This thesis focuses on imaging innovations with iron-oxides and fluorine based contrast media for vascular MRI. Although the research performed was of pre-clinical nature, the ultimate goal is translation and clinical implementation. We found that, with the combination of iron oxide labeled particles and positive contrast techniques, angiogenesis could be visualized in spite of the small size of the target blood vessels. Tumor angiogenesis is a sign of tumor malignancy and molecular MRI, targeted to the activated endothelium, may provide valuable diagnostic information with a minimally invasive technique. The use of iron-oxide contrast media is probably closest to a broad clinical acceptation, as it has already been used in (non-molecular) contrast media MRI applications in clinical studies.

For fluorine contrast media, this thesis shows the strengths and weaknesses of fluorine imaging and its potential in the detection of abnormalities of the pathologic vascular system. However, the absence of clinically available contrast media and the limited availability of clinical fluorine MR acquisition technology (e.g. RF components and pulse sequences) hamper clinical translation. The in vivo detection of fluorine particles with MR is usually based on the fluorine spin density, which requires that the local concentration of fluorine needs to be rather high in comparison to GBCAs or iron-oxide particles. In clinical trials, fluorine is therefore primarily used for human cell tracking studies, where the cells are loaded with fluorine prior to injection. For fluorine imaging, dedicated (radiofrequency) hardware is required, which is also a hurdle to perform fluorine imaging on clinical MR systems. These aspects hamper the translation of experimental fluorine imaging to clinical applications. This thesis shows the potential of fluorine imaging and its potential benefits for the patient. Despite the fact that all essential elements of fluorine MR imaging, such as contrast media and fluorine capable MRI systems are available, major steps still need to be taken to increase the sensitivity of fluorine detection before real clinical applications become feasible. 


\section{References}

1. Kanda, T., K. Ishii, H. Kawaguchi, K. Kitajima, and D. Takenaka, High signal intensity in the dentate nucleus and globus pallidus on unenhanced $T_{1}$-weighted MR images: relationship with increasing cumulative dose of a gadoliniumbased contrast material. Radiology, 2014. 270(3): p. 834-41.

2. Gibby, W.A., K.A. Gibby, and W.A. Gibby, Comparison of Gd DTPA-BMA (Omniscan) versus Gd HP-D03A (ProHance) retention in human bone tissue by inductively coupled plasma atomic emission spectroscopy. Invest Radiol, 2004. 39(3): p. 138-42.

3. Kanal, E. and M.F. Tweedle, Residual or retained gadolinium: practical implications for radiologists and our patients. Radiology, 2015. 275(3): p. 630-4. 


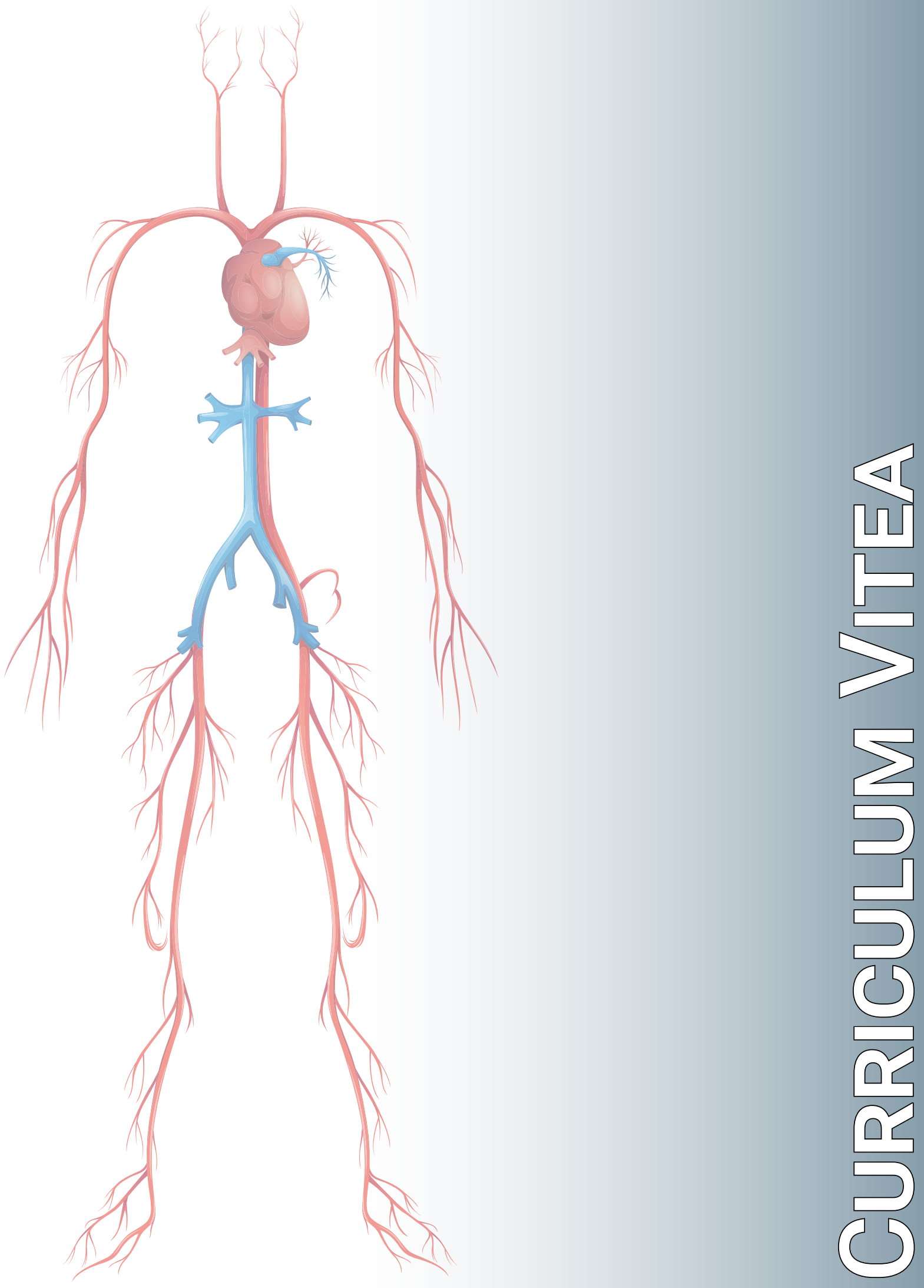




\section{About the author}

Martijn Wolters was born on April 4th, 1981 in Emmen, The Netherlands. After obtaining his general secondary education (MAVO) diploma at Hondsrug College Emmen in 1998, he attended intermediate vocational education (MTS) at Drenthe College in Emmen. Hereceived his diploma as telematics engineer in 2002. Next he studied applied physics at the TH-Rijswijk in Rijswijk. He performed his graduation work at University Medical Center Utrecht on the development of a high resolution single photon counting gammacamera for a small animal SPECT system. He received his Bachelor in Applied Physics in 2006 and in the same year he started the Master

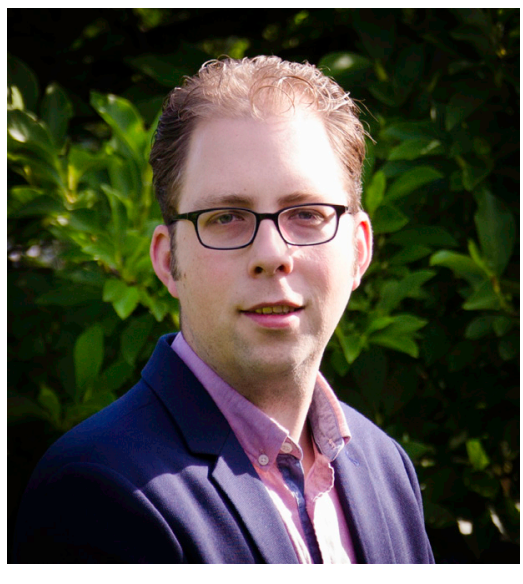
Medical Engineering at the Eindhoven University of Technology. For this graduation project he wrote his master thesis entitled "Molecular susceptibility contrast MRI of tumor angiogenesis with targeted iron oxide nanoparticles" at the Maastricht University Medical Center, department of Radiology. He obtained his Master degree in 2009 and started as a PhD student at the department of Radiology in the same year. Under supervision of dr. M.E. Kooi, prof.dr.ir. W.H. Backes and prof.dr. M.J. Post he studied the applicability of disease specific fluorine and iron oxide nanoparticle contrast agents for MRI. This research focused on the use of these advanced contrast agents in vascular MRI in basic research and on the outlook for clinical translation. As of 2014, Martijn has worked on different assignments as an interim professional in biomedical research and development of medical equipment. Currently he is working for the Engineering Unit of Yacht Eindhoven on an assignment as a system verification engineer at Philips IGT Business Incubation. 


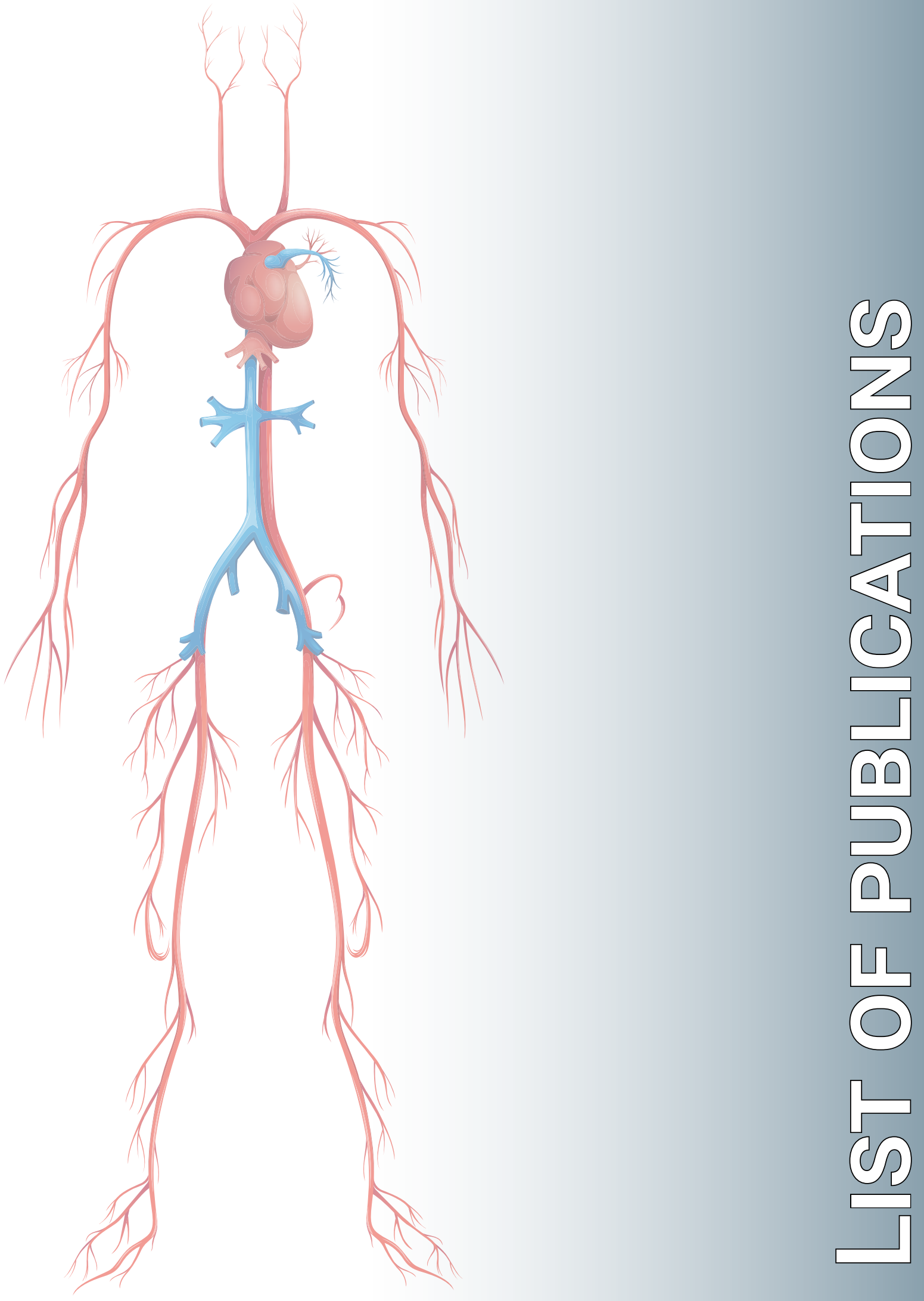




\section{Publications}

M. Wolters, R.H.M. van Hoof, A. Wagenaar, K. Douma, M.A.M.J. van Zandvoort, T.H. Hackeng, M.J. Post, W.H. Backes, M.E. Kooi: MRI artifacts in the ferric chloride thrombus animal model, an alternative solution: Preventing MRI artifacts after thrombus induction with a non-ferromagnetic Lewis acid. Journal of Thrombosis and Haemostasis, 2013

M. Wolters, S.G. Mohades, T.H. Hackeng, M.J. Post, M.E. Kooi, W.H. Backes: Clinical perspectives of hybrid proton-fluorine magnetic resonance imaging and spectroscopy. Investigative Radiology, 2013

M. Wolters, M. Oostendorp, B.F. Coolen, M.J. Post, J.M.H. Janssen, G.J. Strijkers, M.E. Kooi, K. Nicolay, W.H. Backes: Efficacy of positive contrast imaging techniques for molecular MRI of tumor angiogenesis. Contrast Media \& Molecular Imaging, 2012

\section{Conference presentations}

M. Wolters, T.M. Hackeng, M.J. Post, M.E. Kooi, W.H. Backes: ${ }^{19} \mathrm{~F}$ MR Angiography. Invited oral presentation, 25th International Workshop on Magnetic Resonance Angiography, Manhattan New York (USA), August 20-23, 2013

M. Wolters, R.H.M van Hoof, K. Douma, M.A.M.J. van Zandvoort, T.M. Hackeng, M.J. Post, W.H. Backes, M.E. Kooi: MRI artifacts in the ferric chloride thrombus animal model: an alternative solution. Poster presentation, 5th Annual Meeting International Society of Magnetic Resonance (ISMRM) Chapter Benelux, Rotterdam, January 14, 2013

M. Wolters, R.H.M van Hoof, K. Douma, M.A.M.J. van Zandvoort, T.M. Hackeng, M.J. Post, W.H. Backes, M.E. Kooi: MRI artifacts in the ferric chloride thrombus animal model: an alternative solution. Poster presentation, 29th Annual Scientific Meeting European Society for Magnetic Resonance in Medicine and Biology (ESMRMB), Lisbon, October 4-6, 2012

M. Wolters, R.H.M. Hoof, H. Duimel, F. Verheyen, M. Post, T. Hackeng, K. Douma, M.A.M.J. van Zandvoort, W.H. Backes, M.E. Kooi: Detection of early thrombus formation with bioconjugated iron oxide nanoparticles. Poster presentation, 4th Annual Meeting International Society of Magnetic Resonance (ISMRM) Chapter Benelux, Leuven, January 16, 2012

M. Wolters, R.H.M. Hoof, H. Duimel, F. Verheyen, M. Post, T. Hackeng, K. Douma, M.A.M.J. van Zandvoort, W.H. Backes, M.E. Kooi. Detection of early thrombus formation with bioconjugated iron oxide nanoparticles. Oral presentation, 28th Annual Scientific Meeting European Society for Magnetic Resonance in Medicine and Biology (ESMRMB), Leipzig, October 6-8, 2011 
M. Wolters, M. Oostendorp, B.F. Coolen, M.J. Post, G.J. Strijkers, K. Nicolay, W.H. Backes: Molecular susceptibility contrast MRI of tumor angiogenesis with targeted iron oxide nanoparticles Poster presentation, 18th Annual Meeting International Society of Magnetic Resonance (ISMRM), Stockholm, May 1-7, 2010

M. Wolters, M. Oostendorp, B.F. Coolen, M.J. Post, G.J. Strijkers, K. Nicolay, W.H. Backes: Molecular susceptibility contrast MRI of tumor angiogenesis with targeted iron oxide nanoparticles. Oral presentation, NVKF Congres, Woudschoten, April 9, 2010

M. Wolters, M. Oostendorp, B.F. Coolen, M.J. Post, G.J. Strijkers, K. Nicolay, W.H. Backes: Molecular susceptibility contrast MRI of tumor angiogenesis with targeted iron oxide nanoparticles. Oral presentation, 2nd Annual Meeting International Society of Magnetic Resonance (ISMRM) Chapter Benelux, Utrecht, January 18, 2010 
List of publications 